Atratores para equações da onda amortecida em domínios arbitrários 

SERVIÇO DE PÓS-GRADUAÇÃO DO ICMC-USP

Data de Depósito:

Assinatura:

\title{
Atratores para equações da onda amortecida em domínios arbitrários
}

\author{
Ariadne Nogueira \\ Orientadora: Profa. Dra. Maria do Carmo Carbinatto \\ Dissertação apresentada ao Instituto de Ciências \\ Matemáticas e de Computação - ICMC-USP, como \\ parte dos requisitos para obtenção do título de Mestre \\ em Ciências - Matemática . VERSÃO REVISADA
}

USP - São Carlos

Maio de 2013 
Ficha catalográfica elaborada pela Biblioteca Prof. Achille Bassi e Seção Técnica de Informática, ICMC/USP, com os dados fornecidos pelo(a) autor(a)

N778 $\begin{aligned} & \text { Nogueira, Ariadne } \\ & \text { / Ariadne Nogueira; orientadora Maria do Carmo } \\ & \text { Carbinatto. -- São Carlos, 2013. } \\ & \text { 117 p. } \\ & \text { Dissertação (Mestrado - Programa de Pós-Graduação } \\ & \text { em Matemática) -- Instituto de Ciências Matemáticas } \\ & \text { e de Computação, Universidade de São Paulo, 2013. } \\ & \text { 1. atratores globais. 2. equações da onda } \\ & \text { amortecida. 3. equações de evoluça. 4. estimativas } \\ & \text { de truncamento. I. Carbinatto, Maria do Carmo, } \\ & \text { orient. II. Título. }\end{aligned}$


“Todo abismo é navegável a barquinhos de papel." (Guimarães Rosa) 



\section{Agradecimentos}

Agradeço, primeiramente, a Deus pela oportunidade e pela força para passar por mais esse estágio na minha vida. Agradeço muito também a minha família, pela base que me foi dada desde sempre e pelo local de refúgio que me proporciona sempre que necessário, sendo exemplo de amor, união e me inspirando a continuar lutando pelos meus objetivos na vida. Principalmente a minha mãe, por ser meu esteio e meu modelo, com toda sua determinação, força de vontade, humildade, carinho e confiança nas minhas escolhas (muitas vezes confiando mais em mim do que eu mesma poderia).

Agradeço também aos meus amigos, os próximos e os distantes. Sejam aqueles que estiveram aqui comigo e fizeram minha estadia muito mais animada, transformando São Carlos na minha casa por tanto tempo; os de minha cidade natal que faziam meus retornos sempre esperados e proveitosos, mostrando que a vida segue para todos, mas que as raízes, quando bem cuidadas, nunca são deixadas pra trás; assim como os mais distantes, os quais eu sempre agradeço à tecnologia por proporcionar o que muitas vezes a grande distância não nos deixa, que é a presença daqueles que amamos quando precisamos compartilhar algo, sejam notícias, curiosidades ou simplesmente a falta que essa pessoa nos faz. Falo com toda certeza que, sem meus amigos, eu não conseguiria.

Agradeço a minha orientadora, professora Maria do Carmo Carbinatto, pelo cuidado, paciência e profissionalismo com o qual sempre me tratou, me ajudando e me acompanhando nessa tortuosa estrada que foi o meu mestrado. Muito obrigada pelas conversas esclarecedoras, pela troca de experiências e pela confiança. Além de uma grande orientadora neste projeto, fico feliz em dizer que tive um modelo de pessoa e de profissional trabalhando comigo durante todo esse tempo.

Agradeço, por fim, à USP, ao CNPq e à FAPESP pela confiança e pelo apoio financeiro. 



\section{Resumo}

Nesse trabalho apresentamos o estudo do artigo [25] que analisa a existência de atratores globais para uma classe de equações da onda amortecida da forma

$$
\begin{array}{rlrl}
\varepsilon u_{t t}+\alpha(x) u_{t}+\beta(x) u-\sum_{i, j} \partial_{i}\left(a_{i j}(x) \partial_{j} u\right) & =f(x, u), & & x \in \Omega, t \in[0, \infty), \\
u(x, t) & =0, & x \in \partial \Omega, t \in[0, \infty)
\end{array}
$$

definidas em um domínio arbitrário $\Omega$.

Palavras-chave: Atratores globais, equações da onda amortecida, equações de evolução, estimativas de truncamento. 



\section{Abstract}

In this work we describe the results of the paper [25]. In [25] the authors prove existence of global attractors for the following semilinear damped wave equation

$$
\begin{aligned}
\varepsilon u_{t t}+\alpha(x) u_{t}+\beta(x) u-\sum_{i, j} \partial_{i}\left(a_{i j}(x) \partial_{j} u\right) & =f(x, u), & x \in \Omega, t \in[0, \infty), \\
u(x, t) & =0, & x \in \partial \Omega, t \in[0, \infty)
\end{aligned}
$$

on an arbitrary domain $\Omega$.

Key words: Global attractors, damped wave equations, evolution equations, tail-estimates. 



\section{Sumário}

\begin{tabular}{ll}
\hline Introdução & 1
\end{tabular}

\begin{tabular}{lll}
\hline 1 & Preliminares & 3 \\
\hline
\end{tabular}

1.1 Os espaços de Sobolev . . . . . . . . . . . . . . . . . . . . . . 3

1.2 Teoria das distribuições $\ldots \ldots \ldots \ldots \ldots$. . . . . . . . . . . . 7

1.3 A medida de não-compacidade de Kuratowski . . . . . . . . . . . . . . . . 9

1.4 Teoria de $C_{0}$-semigrupos $\ldots \ldots \ldots \ldots \ldots$

1.5 Semifluxos globais e atratores globais $\ldots \ldots \ldots \ldots$

1.6 Problemas semilineares . . . . . . . . . . . . . . . . . . . 21

2 A definição do $C_{0}$-semigrupo

2.1 Resultados preliminares . . . . . . . . . . . . . . . . . 25

2.2 A definição do $C_{0}$-semigrupo associado à equação da onda amortecida $\ldots$. . . . . 29

3 A definição do semifluxo

3.1 Um resultado preliminar $\ldots \ldots \ldots \ldots \ldots$. . . . . . . . . . . 51

3.2 O semifluxo gerado pelas soluções da equação da onda amortecida . . . . . . . 53

$\begin{array}{|ll|}4 & \text { Existência de atrator global }\end{array}$

4.1 Um resultado preliminar $\ldots \ldots \ldots 6$

4.2 Estimativas de truncamento . . . . . . . . . . . . . . . . . . . . . 69

4.3 Compacidade assintótica e existência de atrator global $\ldots \ldots$. . . . . . . . 83

\begin{tabular}{ll}
\hline Considerações finais & 103
\end{tabular} 
\begin{tabular}{lll}
\hline A Demonstrações auxiliares & 105
\end{tabular}

A.1 Demonstração de resultados da Seção 1.5 . . . . . . . . . . . . . . . . . . 105

A.2 Demonstração de resultados da Seção 1.6 . . . . . . . . . . . . . . . . . 110

\begin{tabular}{ll}
\hline Referências bibliográficas & 115
\end{tabular} 


\section{Introdução}

Nesse trabalho estudaremos a existência de atrator global para uma classe de equações de evolução. Nas últimas décadas muito se tem estudado acerca do comportamento assintótico de equações de evolução não-lineares provenientes de equações diferenciais parciais. Uma das ferramentas mais utilizadas para obter informações dessas equações é o conceito de atrator global [18, 22, 9, 28], um conjunto invariante que contêm informações sobre a dinâmica assintótica de uma dada equação de evolução. A existência de um atrator global nos garante que o fenômeno se aproxima de um padrão no futuro. O sucesso desse tipo de abordagem se deu principalmente pela existência de vários modelos, importantes por suas aplicações, onde foi provada a existência de atratores globais e foram feitas análises sobre sua estrutura, comportamento e propriedades [18, 2, 9, 20, 28].

Podemos notar na literatura que há um grande número de trabalhos sobre a existência de atratores globais para equações definidas em domínios limitados. Porém, o estudo da existência de atratores em domínios não limitados traz novas dificuldades. Uma das dificuldades é a ausência de imersão compacta entre vários espaços de funções e tal compacidade é importante para uma solução ter um bom comportamento assintótico. Várias técnicas têm sido desenvolvidas para contornar a perda de compacidade em problemas envolvendo domínios não limitados. Para equações de reação-difusão semilineares podemos citar [1, 3, 24, 29] e para caso de equações da onda amortecida semilineares temos os trabalhos [13, 14, 15, 25].

Nesse trabalho, seguindo o que foi feito no artigo de Prizzi e Rybakowski [25], analisamos a existência de atratores globais para a seguinte classe de equações da onda amortecida

$$
\begin{array}{ll}
\varepsilon u_{t t}+\alpha(x) u_{t}+\beta(x) u-\sum_{i j} \partial_{i}\left(a_{i j}(x) \partial_{j} u\right)=f(x, u), & x \in \Omega, t \in[0, \infty), \\
u(x, t)=0, & x \in \partial \Omega, t \in[0, \infty) .
\end{array}
$$

Aqui, $N \in \mathbb{N}, \Omega$ é um subconjunto aberto arbitrário de $\mathbb{R}^{N}$ (limitado ou não), $\varepsilon>0$ é um parâmetro constante, $\alpha, \beta: \Omega \rightarrow \mathbb{R}$ e $f: \Omega \times \mathbb{R} \rightarrow \mathbb{R}$ são funções dadas e $L u:=$ $\sum_{i j} \partial_{i}\left(a_{i j}(x) \partial_{j} u\right)$ é um operador diferencial de segunda ordem na forma divergente. Observamos que os autores não assumem hipóteses de regularidade em $\partial \Omega, \beta, a_{i j}$ e $f(\cdot, u)$ e consideram 
$f(x, \cdot)$ com crescimento crítico ou subcrítico.

Em [25] os autores assumem que $\alpha \in L^{\infty}(\Omega)$ sendo limitada inferiormente por uma constante positiva e que $L$ seja uniformemente elíptico com coeficientes em $L^{\infty}(\Omega)$. Além disso, assumem também que $\beta \in L_{u}^{p}\left(\mathbb{R}^{N}\right)$ (o espaço $L_{u}^{p}\left(\mathbb{R}^{N}\right)$ é definido na Seção 2.2). Com relação à não linearidade $f: \Omega \times \mathbb{R} \rightarrow \mathbb{R}$, há uma restrição no seu crescimento e uma condição de dissipatividade.

As condições impostas pelos autores em Hipótese 2.5. Hipótese 2.16 e Hipótese 4.3 implicam que as soluções da equação $(E)$ quando considerada como um sistema $(u, v)$, onde $v=u_{t}$, gera um semifluxo global $\pi_{f} \operatorname{em~} Z=H_{0}^{1}(\Omega) \times L^{2}(\Omega)$ (ver Teorema 4.12). Além disso, as condições impostas na Hipótese 4.14 (para o caso subcrítico) e na Hipótese 4.15 (para o caso crítico) garantem a existência de um atrator global associado ao semifluxo $\pi_{f}$ (ver Teore$\operatorname{mas} 4.17 \mathrm{e} 4.21$.

Para mostrar os Teoremas 4.12, 4.17 e 4.21, os autores utilizaram estimativas de truncamento. Este método foi introduzido por Wang em [29] para equações parabólicas definidas em domínios não limitados e foi usado por Fall e You em [13] para estabelecer a existência de atrator global para equações do tipo $(E)$ com hipóteses mais restritas do que as utilizadas por Prizzi e Rybakowski. Observamos que os argumentos utilizados por Feireisl em [14, 15] exigem hipóteses adicionais de regularidade em $f(x, u)$ com relação a todas as variáveis $(x, u)$ e hipóteses de crescimento de $\left|\partial_{u} f(x, 0)\right|$ e $\left|\partial_{x} f(x, 0)\right|$. Mais ainda, em [14, 15] é tratado o caso $\Omega=\mathbb{R}^{3}$ e $L=\Delta$.

Para descrever os resultados obtidos por Prizzi e Rybakowski em [25], este trabalho será organizado como o que segue. No Capítulo 1 introduziremos conceitos e propriedades preliminares importantes que serão utilizados no decorrer do texto, inclusive a proposição que proverá a existência do atrator global ao final do trabalho, ver Proposição1.60. Alguns resultados das Seções 1.5 e 1.6 são demonstrados no Apêndice A.

No Capítulo 2 há o estudo do $C_{0}$-semigrupo associado à solução da equação da onda amortecida $(E)$. Nesse capítulo apresentamos a Hipótese 2.5 e a Hipótese 2.16 que permitirão definir o $C_{0}$-semigrupo em $Z=H_{0}^{1}(\Omega) \times L^{2}(\Omega)$. Já no Capítulo 3 apresentamos as condições sobre a não linearidade $f$ necessárias (ver Proposição 3.10 ) para a existência do semifluxo local $\pi_{f}$ gerado pelas soluções de $(E)$.

Finalmente, no Capítulo 4 mostramos como Hipótese 2.5. Hipótese 2.16 e Hipótese 4.3 implicam que $\pi_{f}$ é semifluxo global em $Z=H_{0}^{1}(\Omega) \times L^{2}(\Omega)$. Concluímos o capítulo apresentando a Hipótese 4.14 (para o caso subcrítico) e a Hipótese 4.15 (para o caso crítico) e mostramos que em ambos os casos, $\pi_{f}$ possui um atrator global. 
Capítulo

1

\section{Preliminares}

Nesse capítulo apresentaremos os conceitos e resultados básicos estudados para a compreensão do texto.

\subsection{Os espaços de Sobolev}

Nesta seção vamos apresentar a definição e algumas propriedades dos espaços de Sobolev para subconjuntos abertos do $\mathbb{R}^{N}, \operatorname{com} n \in \mathbb{N}$. As referências para esta seção são os livros [5] e [8].

Seja $\Omega$ um subconjunto aberto de $\mathbb{R}^{N}$ e seja $p \in \mathbb{R}$ com $1 \leq p \leq \infty$. Iniciaremos com a definição dos espaços $W^{1, p}(\Omega)$ e, para tanto, recordemos que $C_{c}^{\infty}(\Omega)$ denota o conjunto das funções $\varphi \in C^{\infty}(\Omega)$ tais que possuem suporte, denotado por supp $\varphi$, compacto.

Definição 1.1. $O$ espaço de Sobolev $W^{1, p}(\Omega)$ é o conjunto das funções $u \in L^{p}(\Omega)$ tais que existem funções $g_{1}, g_{2}, \ldots, g_{N} \in L^{p}(\Omega)$ com a propriedade

$$
\int_{\Omega} u \frac{\partial \varphi}{\partial x_{i}}=-\int_{\Omega} g_{i} \varphi, \text { para todo } \varphi \in C_{c}^{\infty}(\Omega) \text { e } i \in\{1,2, \ldots, N\} .
$$

Em particular para o caso $p=2$, definimos

$$
H^{1}(\Omega)=W^{1,2}(\Omega)
$$

Se $u \in W^{1, p}(\Omega)$, denotamos $\frac{\partial u}{\partial x_{i}}=g_{i}$, para $i \in\{1,2, \ldots, N\}$, e escrevemos

$$
\nabla u=\left(\frac{\partial u}{\partial x_{1}}, \frac{\partial u}{\partial x_{2}}, \ldots, \frac{\partial u}{\partial x_{N}}\right)
$$


O espaço $W^{1, p}(\Omega)$ é equipado com a norma

$$
|u|_{W^{1, p}(\Omega)}=|u|_{L^{p}(\Omega)}+\sum_{i=1}^{N}\left|\frac{\partial u}{\partial x_{i}}\right|_{L^{p}(\Omega)}, u \in W^{1, p}(\Omega) .
$$

Para $1 \leq p<\infty$, a norma $\|\cdot\|_{W^{1, p}(\Omega)}$ é equivalente à norma

$$
n(u)=\left(|u|_{L^{p}(\Omega)}^{p}+\sum_{i=1}^{N}\left|\frac{\partial u}{\partial x_{i}}\right|_{L^{p}(\Omega)}^{p}\right)^{\frac{1}{p}}, u \in W^{1, p}(\Omega)
$$

Além disso, o espaço $H^{1}(\Omega)$ é equipado com o produto interno

$$
\langle u, v\rangle_{H^{1}(\Omega)}=\langle u, v\rangle_{L^{2}(\Omega)}+\sum_{i=1}^{N}\left\langle\frac{\partial u}{\partial x_{i}}, \frac{\partial v}{\partial x_{i}}\right\rangle_{L^{2}(\Omega)}=\int_{\Omega} u v d x+\sum_{i=1}^{N} \int_{\Omega} \frac{\partial u}{\partial x_{i}} \frac{\partial v}{\partial x_{i}} d x
$$

onde $u, v \in H^{1}(\Omega)$. As propriedades mais elementares dos espaços definidos estão no resultado abaixo:

Proposição 1.2. O espaço de Sobolev $W^{1, p}(\Omega)$ é um espaço de Banach para $1 \leq p \leq \infty$. Se $1<p<\infty, W^{1, p}(\Omega)$ é um espaço reflexivo; $e W^{1, p}(\Omega)$ é um espaço separável para $1 \leq p<\infty$. Além disso, $H^{1}(\Omega)$ é um espaço de Hilbert separável.

Se $u \in C^{1}(\Omega) \cap L^{p}(\Omega)$ e se $\frac{\partial u}{\partial x_{i}} \in L^{p}(\Omega)$, para todo $i \in\{1,2, \ldots, N\}$, então $u \in W^{1, p}(\Omega)$. Desse modo, as derivadas parciais usuais coincidem com as derivadas parciais em $W^{1, p}(\Omega)$, o que torna a notação consistente.

A seguir apresentamos uma caracterização simples das funções em $W^{1, p}(\Omega)$. Antes recordamos a seguinte definição:

Definição 1.3. Seja $\Omega \subset \mathbb{R}^{N}$ um aberto. Dizemos que um conjunto aberto $\omega$ está fortemente incluído em $\Omega$, e denotamos por $\omega \subset \subset \Omega$, se o fecho $\bar{\omega}$ de $\omega$ é um subconjunto de $\Omega$ e $\bar{\omega}$ é um conjunto compacto.

Proposição 1.4. Seja $u \in L^{p}(\Omega)$, com $1<p \leq \infty$. As seguintes afirmativas são equivalentes:

(i) $u \in W^{1, p}(\Omega)$.

(ii) existe uma constante $C \geq 0$ tal que

$$
\left|\int_{\Omega} u \frac{\partial \varphi}{\partial x_{i}} d x\right| \leq C|\varphi|_{L^{p^{\prime}(\Omega)}}, \text { para todo } \varphi \in C_{c}^{\infty}(\Omega) \text { e todo } i \in\{1,2, \ldots, N\},
$$

onde $p^{\prime}:=p /(p-1)$. 
(iii) existe uma constante $C \geq 0$ tal que para todo $\omega \subset \subset \Omega$ e para todo $h \in \mathbb{R}^{N}$, com $|h|<$ $\operatorname{dist}(\omega, \partial \Omega)$, temos que

$$
\left|\tau_{h} u-u\right|_{L^{p}(\Omega)} \leq C|h|
$$

onde $\tau_{h} u(x)=u(x+h), x \in \mathbb{R}$.

Observação 1.5. Se $u \in W^{1, p}(\Omega)$, a constante positiva $C \geq 0$ em (ii) e (iii) na Proposição 1.4 pode ser $C=|\nabla u|_{L^{p}(\Omega)}$.

O próximo resultado generaliza a propriedade do produto para derivação em $W^{1, p}(\Omega)$.

Proposição 1.6 (Diferenciação de produto). Seja $1 \leq p \leq \infty$ e considere funções $u, v \in$ $W^{1, p}(\Omega) \cap L^{\infty}(\Omega)$. Então $u v \in W^{1, p}(\Omega) \cap L^{\infty}(\Omega) e$

$$
\frac{\partial(u v)}{\partial x_{i}}=\frac{\partial u}{\partial x_{i}} v+u \frac{\partial v}{\partial x_{i}}, \text { para todo } i \in\{1,2, \ldots, N\}
$$

Seja $1 \leq p<\infty$. Então $W_{0}^{1, p}(\Omega)$ denota o fecho de $C_{c}^{1}(\Omega)$ em $W^{1, p}(\Omega)$. Definimos também $H_{0}^{1}(\Omega)=W_{0}^{1,2}(\Omega)$.

O espaço $W_{0}^{1, p}(\Omega)$ equipado com a norma de $W^{1, p}(\Omega)$ é um espaço de Banach separável. Além disso, é reflexivo se $1<p<\infty$. O espaço $H_{0}^{1}(\Omega)$, equipado com o produto escalar de $H^{1}(\Omega)$, é um espaço de Hilbert.

Observação 1.7. Como $C_{c}^{1}\left(\mathbb{R}^{N}\right)$ é denso em $W^{1, p}\left(\mathbb{R}^{N}\right)$, temos que $W_{0}^{1, p}\left(\mathbb{R}^{N}\right)=W^{1, p}\left(\mathbb{R}^{N}\right)$. Porém, em geral, se $\Omega \subset \mathbb{R}^{N}$ e $\Omega \neq \mathbb{R}^{N}$, então $W_{0}^{1, p}(\Omega) \neq W^{1, p}(\Omega)$.

Como $C_{c}^{\infty}(\Omega)$ é denso em $W_{0}^{1, p}(\Omega)$, segue que $C_{c}^{\infty}(\Omega)$ pode ser usado na definição de $W_{0}^{1, p}(\Omega)$.

Passamos agora à definição dos espaços de Sobolev $W^{m, p}(\Omega)$, onde $m \in \mathbb{N}$ com $m \geq 2$.

Seja $m \geq 2, m \in \mathbb{N}$, e seja $p$ um número real com $1 \leq p \leq \infty$. Definimos indutivamente

$$
W^{m, p}(\Omega)=\left\{u \in W^{m-1, p}(\Omega) \mid \frac{\partial u}{\partial x_{i}} \in W^{m-1, p}(\Omega), \text { para todo } i \in\{1,2, \ldots, N\}\right\}
$$

Alternativamente, $W^{m, p}(\Omega)$ pode ser caracterizado da seguinte forma: $u \in W^{m, p}(\Omega)$ se, e somente se, $u \in L^{p}(\Omega)$ e para todo $\alpha \operatorname{com}|\alpha| \leq m$ existe um $g_{\alpha} \in L^{p}(\Omega)$ tal que

$$
\int_{\Omega} u D^{\alpha} \varphi=(-1)^{|\alpha|} \int_{\Omega} g_{\alpha} \varphi, \text { para todo } \varphi \in C_{c}^{\infty}(\Omega) .
$$


Observação 1.8. Acima usamos a notação padrão de multi-índice, isto é, $\alpha=\left(\alpha_{1}, \alpha_{2}, \ldots, \alpha_{N}\right)$, onde $\alpha_{i} \in \mathbb{N}$ para todo $i \in\{1,2, \ldots, N\} e|\alpha|=\sum_{i=1}^{N} \alpha_{i}$. Além disso,

$$
D^{\alpha} \varphi=\frac{\partial^{|\alpha|} \varphi}{\partial x_{1}^{\alpha_{1}} \partial x_{2}^{\alpha_{2}} \cdots \partial x_{N}^{\alpha_{N}}}, \text { para } \varphi \in C_{c}^{\infty}(\Omega)
$$

Se $u \in W^{m, p}(\Omega)$, definimos a notação de $D^{\alpha} u=g_{\alpha}$. O espaço $W^{m, p}(\Omega)$, quando equipado com a norma

$$
|u|_{W^{m, p}(\Omega)}=\sum_{0 \leq|\alpha| \leq m}\left|D^{\alpha} u\right|_{L^{p}(\Omega)}, u \in W^{m, p}(\Omega) .
$$

Temos que $\left(W^{m, p}(\Omega),|\cdot|_{W^{m, p}(\Omega)}\right)$ é um espaço de Banach. O espaço $H^{m}(\Omega)=W^{m, 2}(\Omega)$ é um espaço de Hilbert quando equipado com o produto escalar

$$
\langle u, v\rangle_{H^{m}(\Omega)}=\sum_{0 \leq|\alpha| \leq m}\left\langle D^{\alpha} u, D^{\alpha} v\right\rangle_{L^{2}(\Omega)}, u, v \in H^{m}(\Omega)
$$

Observação 1.9. Impondo condições de regularidade em $\Omega$ e supondo que $\Gamma=\partial \Omega$ seja um conjunto limitado, pode ser mostrado que a norma $\|\cdot\|_{W^{m, p}(\Omega)}$ de $W^{m, p}(\Omega)$ é equivalente à

$$
n(u)=|u|_{L^{p}(\Omega)}+\sum_{|\alpha|=m}\left|D^{\alpha} u\right|_{L^{p}(\Omega)}, u \in W^{m, p}(\Omega) .
$$

Concluimos essa seção apresentando algumas desigualdades importantes envolvendo os espaços de Sobolev.

No que segue dado $1 \leq p<N$, definimos $p^{*}:=N p /(N-p)$. O resultado a seguir foi retirado de [8] (ver também [8, Teorema 1.3.1] e [8, Observação 1.3.3]).

Teorema 1.10 (Teorema de Imersão de Sobolev). Seja $\Omega$ um subconjunto aberto de $\mathbb{R}^{N}$.

(i) Suponha que $1 \leq p<N$. Para todo $q \in\left[p, p^{*}\right]$, temos que $W_{0}^{1, p}(\Omega) \subset L^{q}(\Omega)$ e existe uma constante $K_{q} \in(0, \infty)$ tal que

$$
|u|_{L^{q}(\Omega)} \leq K_{q}|u|_{W_{0}^{1, p}(\Omega)}, \text { para todo } u \in W_{0}^{1, p}(\Omega)
$$

(ii) Se $p=N$, então $W_{0}^{1, p}(\Omega) \subset L^{q}(\Omega)$ para todo $q \in[p, \infty)$ e existe uma constante $K_{q} \in(0, \infty)$ tal que

$$
|u|_{L^{q}(\Omega)} \leq K_{q}|u|_{W_{0}^{1, p}(\Omega)}, \text { para todo } u \in W_{0}^{1, p}(\Omega) .
$$

(iii) Se $p>N$, então $W_{0}^{1, p}(\Omega) \subset L^{\infty}(\Omega) \cap C^{0, \alpha}(\Omega)$, onde $\alpha=(p-N) / p$. Além disso, existe 
uma constante $K \in(0, \infty)$ tal que

$$
|u|_{L^{\infty}(\Omega)} \leq K|u|_{W_{0}^{1, p}(\Omega)}, \text { para todo } u \in W_{0}^{1, p}(\Omega)
$$

O resultado a seguir de imersão compacta pode ser encontrado em [5, Teorema 9.16] juntamente com [5, Observação 20].

Teorema 1.11 (Rellich-Kondrachov). Seja $\Omega$ aberto limitado arbitrário de $\mathbb{R}^{N}$.

(i) Se $1 \leq p<N$, para todo $q \in\left[1, p^{*}\right)$, então a inclusão $\imath: W_{0}^{1, p}(\Omega) \rightarrow L^{q}(\Omega)$ é uma aplicação compacta.

(ii) Se $p=N$, então para todo $q \in[p, \infty)$, a inclusão $\imath: W_{0}^{1, p}(\Omega) \rightarrow L^{q}(\Omega)$ é uma aplicação compacta.

(iii) Se $p>N$, então a inclusão $\imath: W_{0}^{1, p}(\Omega) \rightarrow C(\bar{\Omega})$ é uma aplicação compacta.

Concluimos a seção com algumas definições auxiliares. Dados um subconjunto $S \subset \mathbb{R}^{N} \mathrm{e}$ uma função $v: S \rightarrow \mathbb{R}$, denotaremos por $\widetilde{v}: \mathbb{R}^{N} \rightarrow \mathbb{R}$ a extensão de $v$ definida por

$$
\widetilde{v}(x)=\left\{\begin{array}{cl}
v(x), & \text { se } x \in S, \\
0, & \text { se } x \in \mathbb{R} \backslash S
\end{array}\right.
$$

Além disso, no desenvolvimento do texto usaremos a seguinte notação. Dado $N \in \mathbb{N}$,

$$
2^{*}= \begin{cases}\frac{2 N}{N-2}, & \text { se } N \geq 3 \\ \text { um arbitrário } \widetilde{p} \in(0, \infty), & \text { se } N=2 \\ \infty, & \text { se } N=1\end{cases}
$$

\subsection{Teoria das distribuições}

Apresentamos agora uma breve introdução às distribuições. O texto a seguir se baseia em [10].

Seja $\Omega$ um conjunto aberto de $\mathbb{R}^{N}$. É claro que $C_{c}^{\infty}(\Omega)$ é um espaço vetorial sobre $\mathbb{R}$ munido das operações algébricas de funções usuais. Vamos apresentar um conceito de convergência em $C_{c}^{\infty}(\Omega)$.

Seja $\left(\varphi_{n}\right)_{n}$ uma sequência de funções em $C_{c}^{\infty}(\Omega)$. Dizemos que a sequência $\left(\varphi_{n}\right)_{n}$ converge em $C_{c}^{\infty}(\Omega)$ para $\varphi \in \mathscr{D}(\Omega)$ se as seguintes condições são satisfeitas: 
(i) existe um conjunto compacto $K$ em $\Omega$ tal que $\operatorname{supp} \varphi_{n} \subset K$ para todo $n \in \mathbb{N}$;

(ii) para todo multi-índice $\alpha \in \mathbb{N}^{N}$, a sequência $\left(D^{\alpha} \varphi_{n}\right)_{n}$ converge uniformemente em $K$ para $D^{\alpha} \varphi$.

Se $\left(\varphi_{n}\right)_{n}$ converge para $\varphi$ no sentido acima, escrevemos $\varphi_{n} \stackrel{d}{\rightarrow} \varphi$.

O espaço vetorial $C_{c}^{\infty}(\Omega)$ munido da noção de convergência que acabamos de apresentar é representado por $\mathscr{D}(\Omega)$.

Observação 1.12. Os elementos de $\mathscr{D}(\Omega)$ são chamados funções teste.

O espaço dual $\mathscr{D}^{\prime}(\Omega)$ de $\mathscr{D}(\Omega)$ é chamado de espaço das distribuições de $\Omega$. Cada elemento de $\mathscr{D}^{\prime}(\Omega)$ é uma distribuição em $\Omega$. Logo, se $T \in \mathscr{D}^{\prime}(\Omega)$, temos que $T: \mathscr{D}(\Omega) \rightarrow \mathbb{R}$ é um funcional linear contínuo com relação à topologia de $\mathscr{D}(\Omega)$, ou seja, para toda sequência $\left(\varphi_{n}\right)_{n}$ em $C_{c}^{\infty}(\Omega)$ tal que $\varphi_{n} \stackrel{d}{\rightarrow} \varphi$ temos que $T\left(\varphi_{n}\right) \rightarrow T(\varphi)$, quando $n \rightarrow \infty$, em $\mathbb{R}$.

Apresentamos um exemplo de distribuição. Denote por $C_{c}^{0}(\Omega)$ o espaço vetorial das funções contínuas com suporte compacto. Como no espaço $\mathscr{D}(\Omega)$, temos a seguinte noção de convergência em $C_{c}^{0}(\Omega)$. Uma sequência $\left(\varphi_{n}\right)_{n}$ de funções de $C_{c}^{0}(\Omega)$ converge para uma função $\varphi \in C_{c}^{0}(\Omega)$ se (e somente se):

(i) existe um compacto $K$ em $\Omega$ tal que $\operatorname{supp} \varphi_{n} \subset K$, para todo $n \in \mathbb{N}$;

(ii) $\varphi_{n}$ converge uniformemente para $\varphi$ em $K$.

O espaço dos funcionais lineares contínuos de $C_{c}^{0}(\Omega)$ é denotado por $C^{\prime}(\Omega)$.

Observação 1.13. Um elemento de $C^{\prime}(\Omega)$ é também chamado uma medida em $\Omega$.

Recordemos que $L_{l o c}^{1}(\Omega)$ é o espaço de funções integráveis em todo subconjunto compacto de $\Omega$. Para cada $f \in L_{\text {loc }}^{1}(\Omega)$ podemos definir uma medida correspondente $\mu_{f}$ em $\Omega$ por

$$
\mu_{f}(\varphi)=\int_{\Omega} f(x) \varphi(x) d x, \quad \varphi \in C_{0}^{0}(\Omega)
$$

Temos que $\mu_{f} \in C^{\prime}(\Omega)$. A correspondência $f \in L_{l o c}^{1}(\Omega) \mapsto \mu_{f} \in C^{\prime}(\Omega)$ é injetora.

A imagem de $L_{l o c}^{1}(\Omega)$ em $C^{\prime}(\Omega)$ por meio da correspondência acima é chamada o conjunto das medidas absolutamente contínuas em $\Omega$. A função $f$ é chamada a densidade de $\mu_{f}$ com relação à medida de Lebesgue.

É usual identificarmos a medida $\mu_{f}$ e sua densidade $f$. Ou seja, é comum escrever $\mu_{f}=f$ e usar $\mu_{f}(\varphi)=f(\varphi)$ para interpretar (1.1). Com essa notação podemos dizer que a medida $\mu_{f}$ é 
de fato uma função. Além disso, $\mu_{f}$ definindo uma distribuição, dizemos que esta distribuição é a função $f$.

Exemplo 1.14. Seja $1 \leq p \leq \infty$. Como temos que $L^{p}(\Omega)$ está contido em $L_{l o c}^{1}(\Omega)$ por um função injetora contínua, temos que todo elemento de $L^{p}(\Omega)$ define uma distribuição em $\Omega$.

Seja $T \in \mathscr{D}^{\prime}(\Omega)$ e $i \in\{1, \ldots, N\}$. A i-ésima derivada de $T$ no sentido de distribuições, é a distribuição $\partial_{i} T$ em $\Omega$ dada por

$$
\partial_{i} T(\varphi)=-T\left(\partial_{i} \varphi\right), \text { para } \varphi \in \mathscr{D}(\Omega),
$$

onde $\partial_{i} \varphi=\frac{\partial}{\partial x_{i}} \varphi$. Mais geralmente, se $\alpha \in \mathbb{N}^{N}$

$$
\partial_{\alpha} T(\varphi)=(-1)^{|\alpha|} T\left(D^{\alpha} \varphi\right), \operatorname{para} \varphi \in \mathscr{D}(\Omega)
$$

\subsection{A medida de não-compacidade de Kuratowski}

Vamos agora apresentar um conceito muito útil para a caracterização de conjuntos compactos em espaços de Banach. Os conceitos e resultados descritos são como em [11].

Seja $X$ um espaço de Banach e denote por $\mathbb{B}$ a família de todos os subconjuntos limitados de $X$. Se $B \in \mathbb{B}$ não é relativamente compacto, então existe um $\varepsilon>0$ tal que $B$ não pode ser coberto por um número finito de bolas de raio $\varepsilon$ e, portanto, não é possível cobrir $B$ por um número finito de conjuntos com diâmetro menor do que $\varepsilon$. Com isso é natural apresentar o seguinte conceito:

Definição 1.15. A função $m: \mathbb{B} \rightarrow \mathbb{R}^{+}$definida por

$m(B)=\inf \{d>0 \mid B$ admite uma cobertura finita de abertos com diâmetro menor ou igual a $d\}$, onde $B \in \mathbb{B}$, é chamada a medida de não-compacidade de Kuratowski.

Observação 1.16. Em [11] também é apresentado a definição da medida de não-compacidade por bolas. Neste caso, trocamos a cobertura de abertos por uma cobertura de bolas abertas com raio $d$.

A Definição 1.15 não é apenas natural, mas possui propriedades importantes. Algumas são listadas a seguir. 
Proposição 1.17. Seja $X$ um espaço de Banach de dimensão infinita, seja $\mathbb{B}$ a família de todos os subconjuntos limitados de X e seja m a medida de não-compacidade de Kuratowski. Então

(i) $m(B)=0$ se, e somente se, $\bar{B}$ é compacto;

(ii) $m(\lambda B)=|\lambda| m(B)$ e $m\left(B_{1}+B_{2}\right) \leq m\left(B_{1}\right)+m\left(B_{2}\right)$, para todo $\lambda \in \mathbb{R} e B_{1}, B_{2} \in \mathbb{B}$;

(iii) se $B_{1}, B_{2} \in \mathbb{B}$ com $B_{1} \subset B_{2}$ então $m\left(B_{1}\right) \leq m\left(B_{2}\right)$;

(iv) $m\left(B_{1} \cup B_{2}\right)=\max \left\{m\left(B_{1}\right), m\left(B_{2}\right)\right\}$, para todo $B_{1}, B_{2} \in \mathbb{B}$;

(v) $m(\operatorname{co}(B))=m(B)$, onde $\operatorname{co}(B)$ é a envoltória convexa de $B$.

Uma demonstração da Proposição 1.17 pode ser encontrada em [11]. Seja agora um exemplo simples e útil cuja demonstração também está em [11].

Exemplo 1.18. Seja $X$ um espaço de Banach de dimensão infinita e seja $B\left(x_{0}, r\right)$ a bola aberta de centro em $x_{0} \in X$ com raio $r>0$. Segue que $m\left(B\left(x_{0}, r\right)\right)=r m(\overline{B(0,1)})$. Além disso, $m\left(B\left(x_{0}, r\right)\right)=2 r$.

Concluimos essa seção com o seguinte resultado de compacidade.

Teorema 1.19 (Teorema de Mazur). Seja $X$ um espaço de Banach e $A \subset X$ um subconjunto compacto. Então $\overline{\operatorname{co}(A)}$ é um subconjunto compacto de $X$.

\subsection{Teoria de $C_{0}$-semigrupos}

Nesta seção apresentaremos resultados sobre a teoria geral de $C_{0}$-semigrupos. Sugerimos [6] e [17] para os detalhes e demonstrações dos resultados descritos. No que segue $X$ denota um espaço de Banach.

Definição 1.20. Um semigrupo de operadores lineares em $X$ é uma família $\{T(t) \mid t \geq 0\}$ tal que para cada $t \geq 0, T(t)$ é um operador linear limitado, isto é $T(t) \in \mathscr{L}(X)$, tal que

1. $T(0)=I$;

2. $T(s+t)=T(s) T(t)$, para todo $s, t \geq 0$.

Se $\{T(t) \mid t \geq 0\}$ é um semigrupo com $\left\|T(t)-I_{X}\right\|_{\mathscr{L}(X)} \rightarrow 0$ quando $t \rightarrow 0^{+}$, dizemos que $\{T(t) \mid t \geq 0\}$ é um semigrupo uniformemente contínuo. Se $\{T(t) \mid t \geq 0\}$ é um semigrupo com $\|T x-x\|_{X} \rightarrow 0$ quando $t \rightarrow 0^{+}$, para cada $x \in X$, dizemos que $\{T(t) \mid t \geq 0\}$ é um $C_{0}$-semigrupo. 
Um $C_{0}$-semigrupo $\{T(t) \mid t \geq 0\}$ é um $C_{0}$-semigrupo de contrações se para cada $t \geq 0, T(t)$ é uma contração, isto é, $\|T(t)\|_{\mathscr{L}(X)} \leq 1$.

O estudo de semigrupos de operadores lineares está diretamente associado ao estudo de problemas de Cauchy lineares da forma

$$
x^{\prime}(t)=A x(t), \quad x(0)=x_{0},
$$

onde $A: D(A) \subset X \rightarrow X$ é um operador linear (em geral não limitado) e $x_{0} \in X$. Se para cada $x_{0} \in X$ o problema de Cauchy (1.2) possui uma única solução, então as soluções de (1.2) geram um o semigrupo $\{T(t) \mid t \geq 0\}$. O próximo exemplo ilustra o caso em que $A \in \mathscr{L}(X)$.

Exemplo 1.21. Seja A um operador linear contínuo em X e considere o problema de Cauchy (1.2). Neste caso, para cada $x_{0} \in X$, existe uma única solução de (1.2) e é dada por

$$
t \in[0, \infty) \mapsto e^{t A} x_{0} \in X
$$

onde $e^{t A}=\sum_{n=0}^{\infty} \frac{(t A)^{n}}{n !}$.

O próximo resultado mostra que todo $C_{0}$-semigrupo possui uma limitação exponencial.

Teorema 1.22. Suponha que $\{T(t) \mid t \geq 0\}$ seja um $C_{0}$-semigrupo. Então existem constantes $M \geq 1$ e $\beta \in \mathbb{R}$ tais que

$$
\|T(t)\|_{\mathscr{L}(X)} \leq M e^{\beta t}, \text { para todo } t \geq 0
$$

Estamos interessados em generalizar as ideias contidas no Exemplo 1.21 para o caso em que o operador $A$ não seja limitado. Antes porém, dado um semigrupo de operadores lineares vamos associá-lo a um problema de Cauchy.

Seja $\{T(t) \mid t \geq 0\}$ um $C_{0}$-semigrupo de operadores lineares. Defina o seguinte conjunto

$$
D=\left\{x \in X \mid \text { o limite } \lim _{t \rightarrow 0^{+}} \frac{T(t) x-x}{t} \text { existe }\right\}
$$

e considere a aplicação definida por $A: D \subset X \rightarrow X$,

$$
A x=\lim _{t \rightarrow 0^{+}} \frac{T(t) x-x}{t}, \text { para } x \in D
$$

Segue que $A$ é um operador linear e seu domínio é $D$. No que segue denotamos $D$ por $D(A)$. O operador $A$ é chamado gerador infinitesimal do $C_{0}$-semigrupo $\{T(t) \mid t \geq 0\}$. 
O próximo resultado traz uma caracterização dos semigrupos uniformemente contínuos por meio de seus geradores infinitesimais.

Teorema 1.23. Seja $\{T(t) \mid t \geq 0\}$ um $C_{0}$-semigrupo. As seguintes afirmativas são equivalentes:

(i) $\{T(t) \mid t \geq 0\}$ é um semigrupo uniformemente contínuo.

(ii) O gerador infinitesimal está definido em todo X.

(iii) Existe um $B \in \mathscr{L}(X)$ tal que $T(t)=e^{t B}$ para todo $t \geq 0$.

Tendo em vista o Teorema 1.23 , notamos a importância de estudarmos $C_{0}$-semigrupos, seus geradores e suas propriedades. Assim, segue o importante resultado.

Teorema 1.24. Suponha que $\{T(t) \mid t \geq 0\}$ seja um $C_{0}$-semigrupo. Então as seguintes propriedades são válidas:

(i) para todo $x \in X$, a aplicação $t \in[0, \infty) \mapsto T(t) x$ é contínua em $[0, \infty)$.

(ii) a aplicação $t \in[0, \infty) \mapsto\|T(t)\|_{\mathscr{L}(X)}$ é semicontínua inferiormente.

(iii) Seja A o gerador infinitesimal de $\{T(t) \mid t \geq 0\}$. Então o conjunto $D(A)$ é denso em $X e$ A é um operador fechado.

(iv) O conjunto $\cap_{m \geq 1} D\left(A^{m}\right)$ é denso em $X$.

(v) Seja $\beta \geq 0$ como no Teorema 1.22 e seja $\lambda \in \mathbb{C}$ tal que $\operatorname{Re}(\lambda)>\beta$. Então $\lambda$ está no conjunto resolvente $\rho(A)$ de $A e$

$$
(\lambda I-A)^{-1} x=\int_{0}^{\infty} e^{-\lambda t} T(t) x d t, \text { para todo } x \in X .
$$

Observação 1.25. Nas condições do Teorema 1.24 temos que a aplicação $t \in[0, \infty) \mapsto$ $\|T(t)\|_{\mathscr{L}(X)}$ é mensurável.

A seguir apresentamos como a teoria de $C_{0}$-semigrupos está relacionada com o problema de Cauchy (1.2):

Proposição 1.26. Seja $X$ um espaço de Banach e seja $\{T(t) \mid t \geq 0\}$ um $C_{0}$-semigrupo de operadores lineares em $X$ com gerador $A: D(A) \subset X \rightarrow X$. Então, para todo $z \in D(A)$, existe uma única função $u:[0, \infty) \rightarrow D(A)$ que é continuamente diferenciável, $u(0)=z e$

$$
u^{\prime}(t)=A u(t), \text { para todo } t \in[0, \infty),
$$

onde u é dado por $u(t)=T(t) z$, para todo $t \in[0, \infty)$. 
Apresentamos também um resultado de unicidade e outro de perturbação de $C_{0}$-semigrupos.

Teorema 1.27. Sejam $\{T(t) \mid t \geq 0\} e\{S(t) \mid t \geq 0\} C_{0}$-semigrupos com geradores infinitesimais $A$ e $B$, respectivamente. Se $A=B$, então $T(t)=S(t)$, para todo $t \geq 0$.

Teorema 1.28. Seja $X$ um espaço de Banach e seja $A: D(A) \subset X \rightarrow X$ o gerador infinitesimal de um $C_{0}$-semigrupo. Suponha que $B: X \rightarrow X$ seja um operador linear limitado de $X$. Então $A+B: D(A) \subset X \rightarrow X$ é o gerador infinitesimal de um $C_{0}$-semigrupo.

O próximo resultado caracteriza os operadores lineares que são geradores infinitesimais de um $C_{0}$-semigrupo.

Teorema 1.29 (Hille-Yosida). Suponha que $A: D(A) \subset X \rightarrow X$ seja um operador linear. As seguintes afirmativas são equivalentes:

(i) A é o gerador infinitesimal de um $C_{0}$-semigrupo $\{T(t) \mid t \geq 0\}$ tal que, para algum $\omega \in \mathbb{R}$,

$$
\|T(t)\|_{\mathscr{L}(X)} \leq e^{\omega t}, \text { para todo } t \geq 0
$$

(ii) A é um operador linear fechado, densamente definido, existe $\omega \in \mathbb{R}$ tal que seu conjunto resolvente contém $(\omega, \infty) e$

$$
\left\|(\lambda I-A)^{-1}\right\|_{\mathscr{L}(X)} \leq \frac{1}{\lambda-\omega}, \text { para todo } \lambda>\omega
$$

Note que as condições $(i)$ e (ii) do Teorema de Hille-Yosida dependem da escolha da norma em $X$. Veremos agora condições para uma generalização do Teorema 1.29 . Antes de enunciar a generalização, apresentamos um resultado auxiliar em sua demonstração.

Lema 1.30. Suponha $A: D(A) \subset X \rightarrow X$ seja um operador linear cujo conjunto resolvente contém o intervalo $(0, \infty)$ e que exista uma constante $M \geq 0$ tal que

$$
\left.\|(\lambda-A)^{-n}\right) \|_{\mathscr{L}(X)} \leq M \lambda^{-n}, \text { para todo } n \in \mathbb{N} \text { e todo } \lambda>0
$$

Então existe uma norma $|\cdot|_{X}$ em $X$ tal que

$$
\|x\|_{X} \leq|x|_{X} \leq M\|x\|_{X}, \text { para todo } x \in X
$$

$e$

$$
\left|(\lambda-A)^{-1} x\right|_{X} \leq \lambda^{-1}|x|_{X}, \text { para todo } x \in X \text { e todo } \lambda>0
$$


Teorema 1.31 (Forma Geral do Teorema de Hille-Yosida). Seja $A: D(A) \subset X \rightarrow X$ um operador linear. As seguintes afirmativas são equivalentes:

(i) A é o gerador infinitesimal de um $C_{0}$-semigrupo $\{T(t) \mid t \geq 0\}$ tal que

$$
\|T(t)\|_{\mathscr{L}(X)} \leq M e^{\beta t}, t \geq 0
$$

onde $M \geq 1 e \beta \in \mathbb{R}$.

(ii) A é um operador fechado, densamente definido, existe um $\beta \in \mathbb{R}$ tal que o conjunto resolvente de A contém o intervalo $(\beta, \infty) e$

$$
\left\|(\lambda I-A)^{-n}\right\|_{\mathscr{L}(X)} \leq M(\lambda-\beta)^{-n}, \text { para todo } \lambda>\beta \text { e } n \in \mathbb{N}
$$

Dado um operador linear $A: D(A) \subset X \rightarrow X$, seu gráfico é denotado por $\mathscr{G}(A)$.

Definição 1.32. Um operador linear $A: D(A) \subset X \rightarrow X$ é um operador fechável se $\overline{\mathscr{G}(A)}$ é gráfico de um operador linear. Um operador linear $\bar{A}: D(\bar{A}) \subset X \rightarrow Y$ é chamado fecho do operador A se $\overline{\mathscr{G}(A)}=\mathscr{G}(\bar{A})$.

Pode ser facilmente mostrado que um operador linear $A$ é um fechável se, e somente se, $(0, y) \in \overline{\mathscr{G}(A)}$ implicar que $y=0$.

Vamos apresentar agora alguns resultados sobre operadores dissipativos. Estes operadores serão importantes mais adiante para o estudo do problema proposto nesse trabalho. Começaremos recordando a definição de operadores dissipativos.

No que segue, o espaço dual de $X$ é denotado por $X^{\prime}$. Se $x \in X$ e $\phi \in X^{\prime}$ no que segue utilizaremos a seguinte notação:

$$
\langle\phi, x\rangle:=\phi(x)
$$

Para cada $x \in X$, defina

$$
\mathscr{J}(x)=\left\{\phi \in X^{\prime} \mid\|\phi\|^{2}=\|x\|^{2}=\langle x, \phi\rangle\right\} .
$$

Segue do Teorema de Hahn-Banach que $\mathscr{J}(x) \neq \emptyset$, para todo $f \in X$. Temos que $\mathscr{J}$ é uma função (multívoca) chamada de aplicação dualidade de X.

Uma aplicação $J: X \rightarrow X^{\prime}$ tal que $J(x) \in \mathscr{J}(x)$ para cada $x \in X$ é chamada uma seção (dual) de $\mathscr{J}$. 
Definição 1.33. Um operador linear $A: D(A) \subset X \rightarrow X$ é chamado dissipativo com respeito a seção dual $J$ se

$$
\operatorname{Re}\langle A x, J(x)\rangle \leq 0 \text {, para todo } x \in D(A) \text {. }
$$

Um operador linear $A: D(A) \subset X \rightarrow X$ é chamado dissipativo se $A$ for dissipativo em relação a alguma seção dual.

Teorema 1.34 (Lumer-Phillips). Suponha que $A: D(A) \subset X \rightarrow X$ seja um operador linear. As seguintes afirmativas são verdadeiras.

(i) Se A é o gerador infinitesimal de $C_{0}$-semigrupo de contrações, então A é um operador linear fechado, densamente definido, dissipativo e $R(\lambda I-A)=X$ para algum $\lambda>0$.

(ii) Se A é um operador dissipativo, $D(A)$ é um conjunto denso em $X$ e $R\left(\lambda_{0} I-A\right)=X$ para algum $\lambda_{0}>0$, então A é o gerador de um $C_{0}$-semigrupo de contrações.

Uma consequência do Teorema de Lumer-Phillips é o seguinte fato que pode ser útil em aplicações. Temos que dado um operador linear $A: D(A) \subset X \rightarrow X$, denotaremos por $A^{\prime}: D\left(A^{\prime}\right) \subset X^{\prime} \rightarrow X^{\prime}$ seu operador adjunto. Com isso, podemos enunciar o seguinte resultado.

Corolário 1.35. Seja $A: D(A) \subset X \rightarrow X$ um operador linear fechado e densamente definido. Se os operadores A e $A^{\prime}$ são dissipativos, então A é o gerador infinitesimal de um $C_{0}$-semigrupo de contrações de $X$.

Teorema 1.36. Seja $A: D(A) \subset X \rightarrow X$ um operador dissipativo em $X$. As seguintes afirmativas são válidas.

(i) Se para algum $\lambda_{0}>0, R\left(\lambda_{0} I-A\right)=X$, então $R(\lambda I-A)=X$ para todo $\lambda>0$.

(ii) Se A é um operador fechável, então seu fecho é um operador dissipativo.

(iii) Se $\overline{D(A)}=X$, então A é um operador fechável.

Teorema 1.37. Seja $A: D(A) \subset X \rightarrow X$ dissipativo com $R(I-A)=X$. Suponha que $X$ seja um espaço reflexivo. Então $D(A)$ é denso em $X$.

Para o caso em que $X$ é um espaço de Hilbert temos a seguinte caracterização de operadores dissipativos.

Proposição 1.38. Seja $(X,\langle\cdot, \cdot\rangle)$ um espaço de Hilbert. Um operador linear $A: D(A) \subset X \rightarrow X$ é dissipativo em $X$ se, e somente se, $\langle A x, x\rangle \leq 0$ para todo $x \in D(A)$. 


\subsection{Semifluxos globais e atratores globais}

Nesta seção introduzimos o conceito de atratores globais para semifluxos globais e apresentamos o resultado da existência. Para detalhes, sugerimos [9], [18] e [7]. Iniciamos com o conceito de semifluxo local.

Nesta seção $X$ é um espaço métrico e sua métrica é denotada por $d$.

Definição 1.39. Seja $D$ um subconjunto aberto em $[0, \infty) \times X$. Uma aplicação contínua $\pi: D \rightarrow$ $X$ é um semifluxo local em $X$ se para todo $x \in X$ existe um $\omega_{x}=\omega_{\pi, x} \in[0, \infty)$ com a seguinte propriedade:

(i) $(t, x) \in D$ se, e somente se, $t \in\left[0, \omega_{x}\right)$;

(ii) $x \pi 0=x$ para todo $x \in X$;

(iii) Para qualquer que seja $(t, x) \in D e(s, x \pi t) \in D$, então $(t+s, x) \in D e$

$$
x \pi(t+s)=(x \pi t) \pi s
$$

onde escrevemos $x \pi t:=\pi(t, x)$ para $(t, x) \in D$.

Um semifluxo global é um semifluxo local com $\omega_{x}=\infty$ para todo $x \in X$.

Dado um intervalo $I$ em $\mathbb{R}$, uma aplicação $\sigma: I \rightarrow X$ é chamada uma solução (de $\pi$ ) se para quaisquer que sejam $t \in I$ e $s \in[0, \infty)$ tais que $t+s \in I$, então $\sigma(t) \pi s$ está bem definido e

$$
\sigma(t) \pi s=\sigma(t+s)
$$

Se $I=\mathbb{R}$, então $\sigma$ é uma solução global de $\pi$ por $x:=\sigma(0)$. Um subconjunto $S$ de $X$ é um conjunto $\pi$-invariante se, para todo $x \in S$, existe uma solução global $\sigma$ de $\pi$ por $x \operatorname{com} \sigma(\mathbb{R}) \subset S$.

Um subconjunto $S$ de $X$ é um conjunto positivamente $\pi$-invariante se, para todo $x \in S$, $x \pi t \in S$ para todo $t \in\left[0, \omega_{x}\right)$.

Uma solução global constante é chamada uma solução estacionária e o seu valor é um ponto de equilíbrio de $\pi$.

Definição 1.40. Seja $\pi$ um semifluxo local em $X$ e seja $N$ um subconjunto de $X$. Dizemos que $\pi$ não explode em $N$ se, para qualquer que seja $x \in X \operatorname{com} x \pi\left[0, \omega_{x}\right) \subset N$, então $\omega_{x}=\infty$. 
No que segue vamos assumir que $\pi$ seja um semifluxo global em $X$. Para cada $t \geq 0$, denote por $\pi_{(t)}$ a aplicação

$$
x \in X \mapsto x \pi t
$$

Seja $x \in X$ e $B$ um subconjunto de $X$. Definimos os seguintes conjuntos:

(i) Para cada $t \in[0, \infty)$, a imagem de $B$ sob $\pi$,

$$
\pi_{(t)}(B)=\{x \pi t \mid x \in B\}
$$

(ii) A órbita positiva de $B$

$$
\gamma^{+}(B)=\bigcup_{t \in[0, \infty)} \pi_{(t)}(B)
$$

(iii) Sejam $t, t^{\prime} \in[0, \infty), \operatorname{com} t<t^{\prime}$. A órbita parcial entre $t$ e $t^{\prime}$ é

$$
\gamma_{\left[t, t^{\prime}\right]}^{+}(B)=\bigcup_{t \leq s \leq t^{\prime}} \pi_{(s)}(B)
$$

(iv) A órbita de $\pi_{(t)}(B)$,

$$
\gamma_{t}^{+}(B)=\bigcup_{s \in[0, \infty)} \pi_{(t+s)}(B) .
$$

Proposição 1.41. Seja $S$ um subconjunto de X.

(a) S é um conjunto $\pi$-invariante se, e somente se, $S=\pi_{(t)}(S)$ para todo $t \in[0, \infty)$.

(b) S é um conjunto positivamente $\pi$-invariante se, e somente se, $\pi_{(t)}(S) \subset S$ para todo $t \in$ $[0, \infty)$.

Seja $x \in X$ e seja $\sigma: \mathbb{R} \rightarrow X$ uma solução global de $\pi$ por $x$. Definimos órbita global relativa à solução global $\sigma$ por $\gamma_{\sigma}(x)=\{\sigma(t) \mid t \in \mathbb{R}\}$. Para cada $t \in \mathbb{R}$, escrevemos $\left(\gamma_{\sigma}\right)_{t}^{-}(x)=\{\sigma(s) \mid$ $s \leq t\}$.

Seja $B$ um subconjunto de $X$. O conjunto $\omega$-limite de $B$ é definido por

$$
\omega(B)=\bigcap_{t \in[0, \infty)} \overline{\gamma_{t}^{+}(B)} .
$$

Seja $x \in X$. Se existe uma solução global $\sigma: \mathbb{R} \rightarrow X$ por $x$, definimos o conjunto $\alpha$-limite de $x$ relativo $a \sigma$ por

$$
\alpha_{\phi}(x)=\bigcap_{t \in(-\infty, 0]} \overline{\left(\gamma_{\phi}\right)_{t}^{-}(x)}
$$


A proposição a seguir apresenta uma caracterização alternativa e útil dos conjunto $\omega$-limites e $\alpha$-limites.

Proposição 1.42. Sejam B um subconjunto de $X$ e $x \in X$. Suponha que exista uma solução global $\sigma: \mathbb{R} \rightarrow X$ de $\pi$ por $x$. As seguintes afirmativas são verdadeiras:

(i) $\omega(B)$ e $\alpha_{\phi}(x)$ são subconjuntos fechados de $X$.

(ii) $y \in \omega(B)$ se, e somente se, existem sequências $\left(t_{n}\right)_{n}$ em $[0, \infty)$ e $\left(x_{n}\right)_{n}$ em B tais que $t_{n} \rightarrow \infty$ e $d\left(x_{n} \pi t_{n}, y\right) \rightarrow 0$ quando $n \rightarrow \infty$.

(iii) $y \in \alpha_{\phi}(x)$ se, e somente se, existe uma sequência $\left(t_{n}\right)_{n}$ em $[0, \infty)$ tal que $t_{n} \rightarrow \infty e$ $d\left(\sigma\left(-t_{n}\right), y\right) \rightarrow 0$ quando $n \rightarrow \infty$.

Definição 1.43. Dizemos que $\pi$ é eventualmente limitado se para cada subconjunto limitado $B$ de $X$ existe um $t_{B} \in[0, \infty)$ tal que $\gamma_{t_{B}}^{+}(B)$ é limitado. Dizemos que $\pi$ é limitado se $\gamma^{+}(B) e ́$ limitado para qualquer que seja o subconjunto limitado $B$ de $X$.

Definiçãa 1.44. Um subconjunto $A$ de $X$ é um atrator global relativo ao semifluxo $\pi$ se A é um subconjunto compacto, invariante e se para todo conjunto limitado $B$ em $X$ e toda vizinhança aberta $U$ de $A$, existe um $t_{B, U} \in[0, \infty)$ tal que $x \pi t \in U$ para todo $x \in B$ e todo $t \in\left[t_{B, U}, \infty\right)$.

Observação 1.45. Observamos que dado um um semifluxo global $\pi$, se existe um atrator global relativo ao semifluxo $\pi$, então é único.

Apresentaremos agora uma caracterização importante de atratores globais.

Proposição 1.46. Suponha que $\pi$ possua um atrator global $\mathscr{A}$. Então

$$
\mathscr{A}=\left\{x \in X \mid \text { existe uma solução global limitada } \sigma_{x}: \mathbb{R} \rightarrow X \text { tal que } \sigma_{x}(0)=x\right\} .
$$

Definição 1.47. Um subconjunto $B$ de $X$ é chamado u-limitado se existe um $t_{B} \in[0, \infty)$ tal que o conjunto $\gamma_{t_{B}}^{+}(B)$ é limitado.

É fácil ver que se $\pi$ é eventualmente limitado, então todo subconjunto limitado de $X$ é u-limitado.

A seguir apresentamos um conceito de compacidade para semifluxos locais. Esta definição é encontrada em [9].

Definição 1.48. Um $\pi$ um semifluxo global em $X$ é assintoticamente compacto se para qualquer que seja $B$ um subconjunto u-limitado de $X,\left(x_{n}\right)_{n}$ um sequência em $B$ e $\left(t_{n}\right)$ uma sequência em $[0, \infty)$, com $t_{n} \rightarrow \infty$ quando $n \rightarrow \infty$, então a sequência $\left(x_{n} \pi t_{n}\right)_{n}$ possui uma subsequência convergente. 
Sejam $A$ e $B$ subconjuntos de $X$. A semidistância de Hausdorff, denotada por $\operatorname{dist}_{H}(A, B)$, é definida por

$$
\operatorname{dist}_{H}(A, B)=\sup _{x \in A} \inf _{y \in B} d(x, y) .
$$

Denotaremos por $\operatorname{dist}(A, B)$ a distância usual dos conjuntos, ou seja,

$$
d(A, B)=\inf _{x \in A} \inf _{y \in B} d(x, y)
$$

Dizemos que o conjunto $A$ atrai o conjunto $B$ sob a ação do semifluxo $\pi$ se

$$
\lim _{t \rightarrow \infty} \operatorname{dist}_{H}\left(\pi_{(t)}(B), A\right)=0 .
$$

Se existir $t_{0} \in[0, \infty)$ tal que $\pi_{(t)}(B) \subset A$, para todo $t \geq t_{0}$, dizemos que o conjunto $A$ absorve $o$ conjunto $B$. Em particular, se $A$ absorve $B$, então $A$ atrai $B$. A recíproca não é verdadeira. Porém o seguinte fato é válido:

Proposição 1.49. Um conjunto A atrai um conjunto $B$ sob a ação do semifluxo $\pi$ se, e somente se, cada vizinhança aberta de A em $X$ absorve o conjunto B.

Demonstração. A demonstração é imediata recordando que uma vizinhança aberta de $A$ é um conjunto da forma $\{x \in X \mid d(x, A)<\varepsilon\}$, para algum $\varepsilon>0$.

A seguir apresentamos o conceito de suavidade assintotica em semifluxos globais e demonstramos uma equivalência importante (ver Proposição 1.51).

Definição 1.50. Um semifluxo global $\pi$ em $X$ assintoticamente suave se para cada subconjunto não-vazio, fechado, limitado e positivamente invariante $W$ de $X$ contém um subconjunto compacto não-vazio $C$ que atrai $W$.

A seguir mostramos a equivalência entre a compacidade assintótica e a suavidade assintótica. A demonstração é apresentada como em [9, Proposição 1.1.3].

Proposição 1.51. Um semifluxo $\pi$ é assintoticamente suave se, e somente se, $\pi$ é assintoticamente compacto.

Uma demonstração da Proposição 1.51 é dada no Apêndice A.

Concluimos essa seção enunciando e demonstrando um resultado de existência de atratores globais para semifluxos. Porém ainda precisamos de algumas definições auxiliares. 
Definição 1.52. Um semifluxo $\pi$ é ponto dissipativo (respectivamente, limitado dissipativo, respectivamente compacto dissipativo) se existe um subconjunto limitado $B$ de $X$ que atrai pontos (respectivamente subconjuntos limitados, respectivamente subconjuntos compactos) de $X$.

Observação 1.53. Na definição acima podemos trocar atrai por absorve sem mudar os significados dos conceitos.

Temos o seguinte resultado de existência de atrator global.

Teorema 1.54. Seja $\pi$ um semifluxo global definido num espaço métrico X. Então $\pi$ possui um atrator global em X se, e somente se, $\pi$ é ponto dissipativo, assintoticamente suave e eventualmente limitado.

A demonstração do Teorema 1.54]pode ser encontra em [8, Teorema 1.1.2]. O Teorema 1.54 é uma consequência dos seguintes lemas cuja demonstrações encontram-se no Apêndice A.

Lema 1.55. Seja $\pi$ um semifluxo global definido num espaço métrico $X$ e suponha que $\pi$ possua um atrator global $\mathscr{A}$ em X. Então $\mathscr{A}$ atrai subconjuntos limitados de X.

Lema 1.56. Seja $\pi$ um semifluxo global definido num espaço métrico $X$ e suponha que $\pi$ possua um atrator global em X. Então $\pi$ é ponto dissipativo, assintoticamente suave e eventualmente limitado.

Lema 1.57. Seja $\pi$ um semifluxo global definido num espaço métrico $X$ e suponha que $\pi$ seja ponto dissipativo, assintoticamente suave e eventualmente limitado. Então existe um conjunto limitado $\mathscr{O} \subset X$ e para cada conjunto compacto $C \subset X$ existe uma vizinhança aberta $\mathscr{N}_{C}$ de $C$ tal que $\mathscr{O}$ absorve $\mathscr{N}_{C}$.

Lema 1.58. Seja $\pi$ um semifluxo global definido num espaço métrico X e suponha que $\pi$ seja ponto dissipativo, assintoticamente suave e eventualmente limitado. Então existe um conjunto compacto e invariante $\mathscr{A}$ que atrai conjuntos limitados de X. Além disso, $\mathscr{A}$ é um atrator global do semifluxo $\pi$.

Demonstração do Teorema 1.54 Segue dos Lemas 1.56 1.58.

Note que, na demonstração do Teorema 1.54 a hipótese de ser ponto dissipativo pode ser substituída por uma condição mais fraca, que para cada $u_{0} \in X$ existe um $t_{u_{0}} \geq 0$ tal que $u_{0} \pi t_{u_{0}} \in$ $B$, sem mudanças no processo de prova do teorema. Logo, temos o seguinte resultado de [9, Corolário 1.1.4] (ver também [25, Proposição 2.2]). 
Corolário 1.59. Seja $\pi$ um semifluxo global definido num espaço métrico X. Então $\pi$ possui um atrator global em $X$ se, e somente se, $\pi$ é assintoticamente suave, eventualmente limitado e para cada subconjunto $B$ de $X$ limitado e para cada $u_{0} \in X$, existe um $t_{u_{0}} \geq 0$ tal que $u_{0} \pi t_{u_{0}} \in B$.

Apresentamos ainda outro resultado de existência de atratores, o qual será utilizado no final deste trabalho para provar o resultado principal deste trabalho:

Proposição 1.60. Um semifluxo global $\pi$ em um espaço métrico X possui um atrator global se, e somente se, as seguintes condições são satisfeitas:

(a) $\pi$ é assintoticamente compacto,

(b) todo subconjunto limitado de X é u-limitado e

(c) existe um conjunto limitado $B_{0}$ em $X$ com a propriedade que para todo $x \in X$ existe um $t_{x} \in[0, \infty)$ tal que $x \pi t_{x} \in B_{0}$.

Demonstração. Segue da Proposição 1.51 que $\pi$ é assintoticamente compacto se, e somente se, assintoticamente suave. Notemos que a condição $(b)$ é equivalente a $\pi$ ser eventualmente limitado. Uma aplicação do Corolário 1.59 completa a demonstração.

\subsection{Problemas semilineares}

Os conceitos apresentados a seguir são baseados no livro [8]. Os enunciados dos resultados abaixo são como em [8], porém não assumimos a hipótese de contração dos $C_{0}$-semigrupos.

Seja $X$ um espaço de Banach. Uma função $F: X \rightarrow X$ é Lipschitziana em subconjuntos limitados de $X$ se, para cada constante $M>0$, existe uma constante $L_{M} \geq 0$ tal que

$$
\|F(y)-F(x)\| \leq L_{M}\|y-x\|, \text { para todo } x, y \in X \operatorname{com}\|x\| \leq M \text { e }\|y\| \leq M \text {. }
$$

Nesta seção $F$ é sempre uma função Lipschitziana em subconjuntos limitados de $X$.

Seja $\{T(t) \mid t \geq 0\}$ um $C_{0}$-semigrupo e seja $A: D(A) \subset X \rightarrow X$ seu gerador infinitesimal. Dado $x \in X$, suponha que exista um $\omega>0$ e uma função $z \in C([0, \omega], D(A)) \cap C^{1}([0, \omega], X)$ tal que

$$
z^{\prime}(t)=A z(t)+F(z(t)), \text { para todo } t \in[0, \omega]
$$


e $z(0)=x$. Ou seja, a função $z$ é uma solução do sistema

$$
\left\{\begin{array}{l}
u^{\prime}(t)=A u(t)+F(u(t)), t \in[0, \omega] \\
u(0)=x
\end{array}\right.
$$

Notemos que a função $z$ também é solução da seguinte equação integral

$$
u(t)=T(t) x+\int_{0}^{t} T(t-s) F(u(s)) d s, \text { para } t \in[0, \omega]
$$

A equação (1.4) é uma versão fraca do problema (1.3). Nesta seção mostraremos um resultado de existência e unicidade de soluções de equações da forma (1.4).

No que segue vamos utilizar o Lema de Gronwall. Recordamos o seu enunciado:

Lema 1.61. [8, Lema de Gronwall] Sejam $T>0, \psi \in L^{1}(0, T)$, com $\psi \geq 0$ quase sempre em $(0, T)$. Considere constantes positivas $C_{1}, C_{2} \geq 0$. Seja $\varphi \in L^{1}(0, T)$, com $\varphi \geq 0$ quase sempre em $(0, T)$, tal que $\psi \varphi \in L^{1}(0, \omega) e$

$$
\varphi(t) \leq C_{1}+C_{2} \int_{0}^{t} \psi(s) \varphi(s) d s, \text { para quase todo } t \in(0, T) .
$$

Então

$$
\varphi(t) \leq C_{1} \exp \left(C_{2} \int_{0}^{t} \psi(s) d s\right), \text { para quase todo } t \in(0, T)
$$

Temos o primeiro resultado de existência de solução para a equação integral (1.4). A demonstração é apresentada no Apêndice A.

Proposição 1.62. Seja $M>0$ e seja $x \in X$ tal que $\|x\| \leq M$. Então existe um $\omega_{M}>0$ e existe uma única solução $u \in C\left(\left[0, \omega_{M}\right], X\right)$ de $[1.4)$ em $\left[0, \omega_{M}\right]$.

Temos ainda um resultado de existência de solução maximal para a equação integral (1.4).

Teorema 1.63. Com as condições apresentadas nesta seção, existe uma função $\omega: X \rightarrow(0, \infty]$ com a seguinte propriedade: para cada $x \in X$ existe uma função $u \in C([0, \omega(x)), X)$ tal que para todo $0<\omega<\omega(x)$, u é a única solução de $(1.4)$ em $C([0, \omega], X)$. Além disso, temos as seguintes alternativas:

(i) $\omega(x)=\infty$ ou

(ii) $\omega(x)<\infty e \lim _{t \rightarrow \omega(x)^{-}}\|u(t)\|=\infty$. 
Concluimos com um resultado de continuidade com relação às condições iniciais.

Proposição 1.64. Com a mesma notação do Teorema 1.63 temos

(i) $\omega: X \rightarrow(0, \infty]$ é semicontínua inferiormente.

(ii) Seja $\left(x_{n}\right)_{n}$ uma sequência em $X$ e $x \in X$ tais que $x_{n} \rightarrow x$ quando $n \rightarrow \infty$ e seja $\omega<\omega(x)$. Para cada $n \in \mathbb{N}$ sejam $u_{n}$ e u as soluções da equação (1.4) correspondentes aos valores iniciais $x_{n}$ e $x$. Então $u_{n} \rightarrow$ u quando $n \rightarrow \infty$ em $C([0, \omega], X)$.

Uma demonstração do Teorema 1.63 e Proposição 1.64 é dada no Apêndice A. 


\section{Capítulo}

\section{A definição do $C_{0}$-semigrupo associado à solução da equação da onda amortecida}

Nesse capítulo apresentaremos o $C_{0}$-semigrupo associado à solução da equação da onda amortecida. Teremos que impor condições (ver Hipótese 2.5 e Hipótese 2.16) ao problema para que o $C_{0}$-semigrupo seja definido. Na seção 2.2 descrevemos sua definição e demonstramos diversas propriedades.

Iniciamos com alguns resultados básicos que serão usados no capítulo.

\subsection{Resultados preliminares}

Iniciamos a seção enunciando um resultado sobre existência de solução de um problema de Cauchy abstrato definido a partir de um $C_{0}$-semigrupo.

Teorema 2.1. Seja $Z$ um espaço de Banach e seja $A: D(A) \subset Z \rightarrow Z$ o gerador infinitesimal de um $C_{0}$-semigrupo $\{T(t) \mid t \in[0, \infty)\}$. Suponha que uma das afirmativas abaixo esteja satisfeita:

(i) $f \in \mathscr{C}([0, \infty), Z)$ tomando valores em $D(A)$ e Af $\in \mathscr{C}\left(\mathbb{R}^{+}, X\right)$ ou

(ii) $f \in \mathscr{C}^{1}([0, \infty), Z)$.

Seja $u_{0} \in D(A)$ e considere o problema de Cauchy abstrato, para $t \in[0, \infty)$ :

$$
\frac{d}{d t} u(t)=A u(t)+f(t), \quad u(0)=u_{0}
$$

O problema (2.1) possui uma única solução $u:[0, \infty) \rightarrow D(A)$ com $u \in \mathscr{C}^{1}([0, \infty), Z)$. Além disso

$$
u(t)=T(t) u_{0}+\int_{0}^{t} T(t-s) f(s) d s, \text { para todo } t \geq 0
$$


Uma demonstração do teorema acima pode ser encontrada em [17, Teorema II.1.3].

Demonstraremos agora o seguinte resultado de análise em espaços de Banach, que será usado na próxima proposição.

Lema 2.2. Seja $X$ um espaço de Banach. Considere $S:[a, b] \rightarrow X$ operador linear diferenciável em $(a, b)$ e $v: X \rightarrow Y$ uma aplicação linear limitada. Então $v S:[a, b] \rightarrow Y$ é uma aplicação diferenciável em $(a, b)$ e vale

$$
\frac{d}{d t}(v S(t))=v\left(\frac{d}{d t} S(t)\right), \text { para todo } t \in(a, b)
$$

Demonstração. Seja $t_{0} \in(a, b)$ fixado arbitrariamente. Temos que

$$
S^{\prime}\left(t_{0}\right)=\lim _{h \rightarrow 0} \frac{S\left(t_{0}+h\right)-S\left(t_{0}\right)}{h} .
$$

A linearidade de $v$ implica que

$$
\frac{v\left(S\left(t_{0}+h\right)\right)-v\left(S\left(t_{0}\right)\right)}{h}=v\left(\frac{S\left(t_{0}+h\right)-S\left(t_{0}\right)}{h}\right) .
$$

Como $v$ é uma aplicação linear limitada e $\left.\frac{d}{d t} S(t)\right|_{t=t_{0}}$ existe, temos

$$
\lim _{h \rightarrow 0}\left(\frac{v\left(S\left(t_{0}+h\right)\right)-v\left(S\left(t_{0}\right)\right)}{h}\right)=v\left(\lim _{h \rightarrow 0} \frac{S\left(t_{0}+h\right)-S\left(t_{0}\right)}{h}\right) .
$$

Portanto, a aplicação $t \in[a, b] \mapsto v(S(t)) \in Y$ é diferenciável em $(a, b)$ e $\left.\frac{d}{d t} v(S(t))\right|_{t=t_{0}}=v\left(S^{\prime}\left(t_{0}\right)\right)$. A demonstração está completa.

Demonstremos agora um resultado sobre $C_{0}$-semigrupos a ser usado no estudo da versão abstrata das equações da onda amortecida.

Proposição 2.3. Sejam $Z$ e $Y$ espaços de Banach e sejam $\left\{S_{Z}(t) \mid t \in[0, \infty)\right\}$ e $\left\{S_{Y}(t) \mid t \in[0, \infty)\right\}$ $C_{0}$-semigrupos definidos em $Z$ e $Y$, respectivamente, com geradores infinitesimais $C_{Z}: D\left(C_{Z}\right) \rightarrow$ $Z$ e $C_{Y}: D\left(C_{Y}\right) \rightarrow Y$, respectivamente. Seja $v: Z \rightarrow Y$ uma aplicação linear limitada com $v\left(D\left(C_{Z}\right)\right) \subset D\left(C_{Y}\right)$. Se $v\left(C_{Z} z\right)=C_{Y}(v(z))$, para todo $z \in D\left(C_{Z}\right)$, então

$$
v\left(S_{Z}(t) z\right)=S_{Y}(t)(v(z)), \text { para todo } z \in Z \text { e para todo } t \in[0, \infty)
$$


Demonstração. Suponha que $z \in D\left(C_{Z}\right)$ e considere o problema de Cauchy linear:

$$
w^{\prime}(t)=C_{Y} w(t), \quad w(0)=v(z)
$$

A Proposição 1.26 implica que o problema (2.3) possui uma única solução $w(t)=S_{Y}(t) v(z)$ para $t \geq 0$. Defina agora

$$
u(t)=v\left(S_{Z}(t) z\right) \text { para } t \geq 0
$$

Notemos que, novamente uma aplicação da Proposição 1.26 implica que $t \in[0, \infty) \mapsto S_{Z}(t) z$ é a única solução do problema de Cauchy linear

$$
f^{\prime}(t)=C_{Z} f(t), \quad f(0)=z
$$

Utilizando o Lema 2.2 para derivar a aplicação $u$ definida em (2.4), obtemos:

$$
u^{\prime}(t)=v\left(\frac{d}{d t} S_{Z}(t) z\right)=v\left(C_{Z} S_{Z}(t) z\right)=C_{Y}\left(v\left(S_{Z}(t) z\right)\right)=C_{Y} u(t), \text { para } t \geq 0
$$

Além disso, $u(0)=v\left(S_{Z}(0) z\right)=v z$. Portanto, a unicidade de soluções implica que $u(t)=w(t)$ para todo $t \geq 0$. Ou seja,

$$
S_{Y}(t) v z=v\left(S_{Z}(t) z\right), \text { para } t \geq 0
$$

Agora seja $z \in Z$. Segue da densidade de $D\left(C_{Z}\right)$ em $Z$ existe uma sequência $\left(z_{n}\right)_{n}$ em $D\left(C_{Z}\right)$ tal que $z_{n} \rightarrow z$ quando $n \rightarrow \infty$. Temos que a relação (2.2) é válida para todo $n \in \mathbb{N}$ e $t \geq 0$. Seja $t \geq 0$, a continuidade da aplicação $z \in Z \mapsto S_{Y}(t) v z$ implica que

$$
S_{Y}(t) v\left(z_{n}\right) \rightarrow S_{Y}(t) v(z), \text { quando } n \rightarrow \infty
$$

Como $v$ é uma aplicação linear contínua temos que

$$
S_{Y}(t) v\left(z_{n}\right)=v\left(S_{Y}(t) z_{n}\right) \rightarrow v\left(S_{Y}(t) z\right), \text { quando } n \rightarrow \infty
$$

Logo, $S_{Y}(t) v(z)=v\left(S_{Y}(t) z\right)$, concluindo a demonstração do resultado.

A seguir demonstramos o seguinte resultado sobre perturbação de $C_{0}$-semigrupos.

Teorema 2.4. Seja $Z$ um espaço de Banach e seja $\left\{S_{Z}(t) \mid t \in[0, \infty)\right\}$ um $C_{0}$-semigrupo de operadores lineares em $Z$ com gerador infinitesimal $C_{Z}: D\left(C_{Z}\right) \rightarrow Z$. Seja $Q: Z \rightarrow Z$ um operador linear e limitado em $Z$. Então o operador linear $C_{Z}+Q: D\left(C_{Z}\right) \rightarrow Z$ é gerador infinitesimal de 
um $C_{0}$-semigrupo $\left\{T_{Z}(t) \mid t \in[0, \infty)\right\}$ de operadores lineares em Z. Além disso,

$$
T_{Z}(t) z=S_{Z}(t) z+\int_{0}^{t} S_{Z}(t-s) Q T_{Z}(s) z d s, \text { para todo } t \geq 0 \text { e } z \in Z .
$$

Demonstração. A primeira afirmação é uma consequência imediata do Teorema 1.28. Denote por $\left\{T_{Z}(t) \mid t \in[0, \infty)\right\}$ o $C_{0}$-semigrupo gerado por $C_{Z}+Q$. Sejam $z \in D\left(C_{Z}+Q\right)$ e $t \in[0, \infty)$. Considere o seguinte problema de Cauchy linear

$$
v^{\prime}(t)=\left(C_{Z}+Q\right) v(t), \quad v(0)=z
$$

Segue da Proposição 1.26 que existe uma única solução para (2.7) e esta solução é dada por

$$
v(t)=T_{Z}(t) z, t \geq 0
$$

Considere agora o seguinte problema de Cauchy abstrato:

$$
u^{\prime}(t)=C_{Z} u(t)+Q T_{Z}(t) z, \quad u(0)=z
$$

Como $Q T_{Z}(t) z \in \mathscr{C}^{1}([0, \infty), Z)$, o Teorema 2.1 implica que o problema (2.8) possui uma única solução $u$ em $[0, \infty)$ e é dada por

$$
u(t)=S_{Z}(T) z+\int_{0}^{t} S_{Z}(t-s) Q T_{Z}(s) z d s, \quad t \geq 0 .
$$

Por outro lado, note que $v(0)=z$ e

$$
v^{\prime}(t)=\frac{d}{d t} T_{Z}(t) z=\left(C_{Z}+Q\right) T_{Z}(t) z=C_{Z} T_{Z}(t) z+Q T_{Z}(t) z=C_{Z} v(t)+Q T_{Z}(t) z
$$

A unicidade de soluções do problema (2.8), implica que $u(t)=v(t)$ para $t \geq 0$. Ou seja,

$$
T_{Z}(t) z=S_{Z}(T) z+\int_{0}^{t} S_{Z}(t-s) Q T_{Z}(s) z d s, t \geq 0
$$

o que completa a demonstração. 


\subsection{A definição do $C_{0}$-semigrupo associado à equação da onda amortecida}

Para o resto desse texto, consideramos $N$ um número natural com $N \geq 1$ e $\Omega$ denotará um subconjunto aberto arbitrário (limitado ou não) de $\mathbb{R}^{N}$. Além disso, $\langle\cdot, \cdot\rangle$ denotará o produto interno usual em $L^{2}(\Omega)$. Vamos assumir as seguintes hipóteses:

Hipótese 2.5. (a) $a_{0}, a_{1} \in(0, \infty)$ são constantes e $a_{i j}: \Omega \rightarrow \mathbb{R}, i, j \in\{1, \ldots, N\}$, são funções em $L^{\infty}(\Omega)$ tais que $a_{i j}=a_{j i}$, para $i, j \in\{1, \ldots N\}$ e

$$
a_{0}|\xi|^{2} \leq \sum_{i, j=1}^{N} a_{i j}(x) \xi_{i} \xi_{j} \leq a_{1}|\xi|^{2} \text {, para todo } \xi \in \mathbb{R}^{N} \text { e quase todo } x \in \Omega
$$

Defina $A(x):=\left(a_{i j}(x)\right)_{i, j=1}^{N}, x \in \Omega$.

(b) $\beta: \Omega \rightarrow \mathbb{R}$ é uma função mensurável com a propriedade

(i) para todo $\bar{\varepsilon} \in(0, \infty)$, existe um $C_{\bar{\varepsilon}} \in[0, \infty)$, com

$$
\left.\left.|| \beta\right|^{1 / 2} u\right|_{L^{2}(\Omega)} ^{2} \leq \bar{\varepsilon}|u|_{H^{1}(\Omega)}^{2}+C_{\bar{\varepsilon}}|u|_{L^{2}(\Omega)}^{2}, \text { para todo } u \in H_{0}^{1}(\Omega)
$$

(ii) $\lambda_{1}:=\inf \left\{\langle A \nabla u, \nabla u\rangle+\left.\langle\beta u, u\rangle\left|u \in H_{0}^{1}(\Omega),\right| u\right|_{L^{2}(\Omega)}=1\right\}>0$.

Note que segue das condições da Hipótese 2.5(a) que $\langle A \nabla u, \nabla u\rangle$ está definido para $u \in H_{0}^{1}(\Omega)$ e que as condições da Hipótese 2.5 (i) implicam que $\langle\beta u, u\rangle$ está definido para $u \in H_{0}^{1}(\Omega)$. Logo, $\langle A \nabla u, \nabla u\rangle+\langle\beta u, u\rangle$ está bem definido para $u \in H_{0}^{1}(\Omega)$.

Lema 2.6. Suponha que as condições da Hipótese 2.5 estejam verificadas. Então

$$
a_{0} \leq a_{i j} \leq a_{1} \text {, para todo } i, j \in\{1, \ldots, N\} \text {. }
$$

Demonstração. Para ver isto, basta tomar $\xi_{i, j} \in \mathbb{R}^{N}, i, j \in\{1, \ldots, N\}$, tal que, dados $i, j \in$ $\{1, \ldots, N\}$, a $i$-ésima e a $j$-ésima coordenadas de $\xi_{i, j}$ são 1 , enquanto é 0 nas outras entradas e usar Hipótese 2.5 $(a)$.

Antes de prosseguir é interessante saber se existem funções que satisfaçam a condição de Hipótese 2.5. (a). Para apresentar uma classe de funções que verificam essas condições, recordemos a definição do espaço das funções localmente uniformes. A definição dada a seguir é como em [1] e também é encontrada em [27]. 
Seja $1<p<\infty$. Definimos o espaço das funções localmente uniforme $L_{u}^{p}(\Omega)$ como o conjunto das funções mensuráveis $\omega: \Omega \rightarrow \mathbb{R}$ tais que

$$
\sup _{y \in \Omega} \int_{B(y) \cap \Omega}|\omega(x)|^{p} d x<\infty
$$

onde, para $y \in \Omega, B(y)$ representa o cubo aberto unitário de $\mathbb{R}^{N}$ centrado em $y$. Temos que $L_{u}^{p}(\Omega)$ é um espaço normado munido com a norma

$$
|\omega|_{L_{u}^{p}(\Omega)}:=\sup _{y \in \Omega}|\omega|_{L^{p}(B(y) \cap \Omega)}
$$

Notemos que $L_{u}^{p}(\Omega)$ contém $L^{\infty}(\Omega), L^{r}(\Omega)$ e $L_{u}^{r}(\Omega)$ para todo $r \geq p$.

Observamos ainda que a escolha de cubos unitários para a definição dos espaços $L_{u}^{p}(\Omega)$ é técnica. Outras escolhas para os raios fornecem normas equivalentes em $L_{u}^{p}(\Omega)$.

O próximo lema mostra condições que garantem que uma dada função $\beta$ satisfaça a Hipótese 2.5(a).

Proposição 2.7. Seja $p \in(1, \infty)$ e seja $\beta: \Omega \rightarrow \mathbb{R}$ tal que $\widetilde{\beta} \in L_{u}^{p}\left(\mathbb{R}^{N}\right)$.

(a) Se $p \geq N / 2$, então existe uma constante $C \in(0, \infty)$ tal que

$$
\left.\left.|| \beta\right|^{1 / 2} u\right|_{L^{2}(\Omega)} \leq C|u|_{H^{1}(\Omega)}, \text { para todo } u \in H_{0}^{1}(\Omega) .
$$

(b) Se $p>N / 2$, então para todo $\bar{\varepsilon} \in(0, \infty)$, existe uma constante $C_{\bar{\varepsilon}} \in(0, \infty)$, tal que

$$
\|\left.\left.\beta\right|^{1 / 2} u\right|_{L^{2}(\Omega)} ^{2} \leq \bar{\varepsilon}|u|_{H^{1}(\Omega)}^{2}+C_{\bar{\varepsilon}}|u|_{L^{2}(\Omega)}^{2}, \text { para todo } u \in H_{0}^{1}(\Omega)
$$

Para a demonstração desse lema, usaremos dois resultados clássicos enunciados a seguir.

Lema 2.8 (Desigualdade de Young). Sejam p e q são números reais positivos tais que $1 / p+$ $1 / q=1$. Para todo par de números reais $a>0$ e $b>0$ vale a desigualdade:

$$
a b \leq \frac{a^{p}}{p}+\frac{b^{q}}{q} .
$$

Lema 2.9 (Desigualdade de Interpolação em $L^{p}$ ). Seja $B \subset \mathbb{R}^{N}$ um conjunto mensurável e seja $f \in L^{p}(B) \cap L^{q}(B)$, com $1 \leq p \leq q \leq \infty$. Então para todo $r$ com $p \leq r \leq q$, temos que $f \in L^{r}(B)$ 
$e$ vale a seguinte desigualdade de interpolação: dado $\theta \in[0,1]$, temos que

$$
|f|_{L^{r}(B)} \leq|f|_{L^{p}(B)}^{\theta}|f|_{L^{q}(B)}^{1-\theta}, \text { onde } \frac{1}{r}=\frac{\theta}{p}+\frac{1-\theta}{q} .
$$

Com isso, voltamos à demonstração.

Demonstração da Proposição 2.7 Seja $\left\{B_{j}\right\}_{j \in \mathbb{N}}$ a família enumerável de cubos centrados nos pontos de coordenadas inteiras em $\mathbb{R}^{N}$ e com lados de comprimento 1 paralelos aos eixos coordenados. Notemos que $\mathbb{R}^{N}=\cup_{j \in \mathbb{N}} \overline{B_{j}}$ e que $\overline{B_{i}} \cap \overline{B_{j}}$ é um conjunto de medida nula para quaisquer que sejam $i, j \in \mathbb{N} \operatorname{com} i \neq j$.

Dado $p \in(1, \infty)$, defina $p^{\prime}=p /(p-1)$. Seja $B$ o cubo de centro na origem de $\mathbb{R}^{N}$ e lado 1 .

Suponha que $p \geq N / 2$. Afirmamos que existe uma constante $M=M(p, N)>0$ tal que

$$
|u|_{L^{2 p^{\prime}(B)}} \leq M|u|_{H^{1}(B)}, \text { para todo } u \in H^{1}(B)
$$

Se $N \geq 3$, como $\frac{1}{p}+\frac{1}{p^{\prime}}=1$ temos que

$$
\frac{1}{p^{\prime}}=1-\frac{1}{p} \leq 1-\frac{2}{N}=\frac{N-2}{N}
$$

e, assim, $p^{\prime} \leq N /(N-2)$. Sendo $2^{*}=2 N /(N-2)$, segue que $2 p^{\prime} \leq 2^{*}$. O Teorema 1.10 implica que existe uma constante $\widetilde{M} \geq 0$ tal que

$$
|u|_{L^{2^{*}(B)}} \leq \widetilde{M}|u|_{H^{1}(B)}, \text { para todo } u \in H^{1}(B)
$$

Como $B$ é um conjunto de medida finita, então $L^{2^{*}}(B) \subset L^{2 p^{\prime}}(B)$ e segue que existe uma constante $M=M(p, 3) \geq 0$ tal que

$$
|u|_{L^{2 p^{\prime}(B)}} \leq M|u|_{H^{1}(B)} \text {, para todo } u \in H^{1}(B)
$$

Se $N=2$ podemos tomar $p^{*}=2 p^{\prime}$ no Teorema 1.10 e, portanto, existe uma constante $M=$ $M(p, 2) \geq 0$ tal que

$$
|u|_{L^{2 p^{\prime}(B)}} \leq M|u|_{H^{1}(B)} \text {, para todo } u \in H^{1}(B) \text {. }
$$

Finalmente, se $N=1$, segue do Teorema 1.10 que existe uma constante $\bar{M} \geq 0$ tal que

$$
|u|_{L^{\infty}(B)} \leq \bar{M}|u|_{H^{1}(B)} \text {, para todo } u \in H^{1}(B) .
$$


Como $B$ é um conjunto de medida finita, $L^{\infty}(B) \subset L^{2 p^{\prime}}(B)$. Segue que existe uma constante $M=M(p, 1) \geq 0$ tal que

$$
|u|_{L^{2 p^{\prime}(B)}} \leq M|u|_{H^{1}(B)} \text {, para todo } u \in H^{1}(B) .
$$

Logo, a afirmativa está demonstrada.

Seja $B(y)$ o cubo de lado 1 centrado em $y \in \mathbb{R}^{N}$. Como $B(y)$ é a imagem de $B$ pela translação, segue que

$$
|u|_{L^{2 p^{\prime}(B(y))}} \leq M|u|_{H^{1}(B(y))} \text {, para todo } u \in H^{1}(B) \text { e para todo } y \in \mathbb{R}^{N} \text {. }
$$

Seja $u \in H_{0}^{1}(\Omega)$. Temos

$$
\begin{aligned}
\int_{\Omega}\left|\beta(x) u^{2}(x)\right| d x & =\int_{\mathbb{R}^{N}}\left|\widetilde{\beta}(x) \widetilde{u}^{2}(x)\right| d x=\sum_{j \in \mathbb{N}} \int_{B_{j}}\left|\widetilde{\beta}(x) \widetilde{u}^{2}(x)\right| d x \\
& \leq \sum_{j \in \mathbb{N}}\left(\int_{B_{j}}|\widetilde{\beta}(x)|^{p} d x\right)^{1 / p}\left(\int_{B_{j}}|\widetilde{u}(x)|^{2 p^{\prime}} d x\right)^{1 / p^{\prime}} \\
& \leq|\widetilde{\beta}|_{L_{u}^{p}\left(\mathbb{R}^{N}\right)} \sum_{j \in \mathbb{N}}\left(\int_{B_{j}}|\widetilde{u}(x)|^{2 p^{\prime}} d x\right)^{1 / p^{\prime}} \\
& \leq\left.|\widetilde{\beta}|_{L_{u}^{p}\left(\mathbb{R}^{N}\right)} M^{2} \sum_{j \in \mathbb{N}}|\widetilde{u}|_{B_{j}}\right|_{H^{1}\left(B_{j}\right)} ^{2}=|\widetilde{\beta}|_{L_{u}^{p}\left(\mathbb{R}^{N}\right)} M^{2} \sum_{j \in \mathbb{N}} \int_{B_{j}}\left(|\nabla \widetilde{u}(x)|^{2}+|\widetilde{u}(x)|^{2}\right) d x \\
& =M^{2}|\widetilde{\beta}|_{L_{u}^{p}\left(\mathbb{R}^{N}\right)}|\widetilde{u}|_{H^{1}\left(\mathbb{R}^{N}\right)}^{2}=M^{2}|\widetilde{\beta}|_{L_{u}^{p}\left(\mathbb{R}^{N}\right)}|u|_{H^{1}(\Omega)}^{2} .
\end{aligned}
$$

Defina $C:=M|\widetilde{\beta}|_{L_{u}^{p}\left(\mathbb{R}^{N}\right)}^{1 / 2}$. A parte $(a)$ do lema está demonstrada.

Suponha agora que $p>N / 2$. Temos que existe um $q=q(p, N) \in(0, \infty)$ tal que $2 p^{\prime}<q<2^{*}$. De fato, se $N \geq 3$, temos que $2 p^{\prime}<2^{*}$ e então existe um $q \in(0, \infty)$ tal que $2 p^{\prime}<q<2^{*}$. Se $N=2$, seja $r>0$ com $2 p^{\prime}<r$ e defina $2^{*}=r$. Novamente, então existe um $q \in(0, \infty)$ tal que $2 p^{\prime}<q<2^{*}$. Finalmente, se $N=1$ temos que $2^{*}=\infty$. Logo, basta tomar um $q \in(0, \infty)$ tal que $2 p^{\prime}<q<\infty$.

Procedendo como anteriormente, temos que existe uma constante $M^{\prime}=M^{\prime}(p, N) \geq 0$ tal que

$$
|u|_{L^{q}\left(B_{j}\right)} \leq M^{\prime}|u|_{H^{1}\left(B_{j}\right)}, \text { para todo } u \in H^{1}\left(B_{j}\right)
$$

Notemos que a constante $M^{\prime}$ independe de $j \in \mathbb{N}$.

Afirmamos que para todo $\widetilde{\varepsilon} \in(0, \infty)$, existe uma constante $C_{\widetilde{\varepsilon}} \in[0, \infty)$ tal que, para todo 
$j \in \mathbb{N}$,

$$
\begin{aligned}
& \left(\int_{B_{j}}|\widetilde{u}(x)|^{2 p^{\prime}} d x\right)^{1 / 2 p^{\prime}} \leq \bar{\varepsilon}\left(\int_{B_{j}}|\widetilde{u}(x)|^{q} d x\right)^{1 / q}+C_{\bar{\varepsilon}}\left(\int_{B_{j}}|\widetilde{u}(x)|^{2} d x\right)^{1 / 2} \\
& \leq\left.\bar{\varepsilon} M^{\prime}|\widetilde{u}|_{B_{j}}\right|_{H^{1}\left(B_{j}\right)} ^{2}+\left.C_{\bar{\varepsilon}}|\widetilde{u}|_{B_{j}}\right|_{L^{2}\left(B_{j}\right)} ^{2} \text {, para todo } u \in H_{0}^{1}(\Omega) \text {. }
\end{aligned}
$$

De fato, seja $u \in H_{0}^{1}(\Omega)$. Sendo $p>1$, então $p^{\prime}>1$. Assim temos que $2<2 p^{\prime}<q$, para todo $N \in \mathbb{N}$. Defina

$$
\theta=\frac{q-2 p^{\prime}}{p^{\prime}(q-2)}
$$

Segue que $\theta \in[0,1] \mathrm{e}$

$$
\frac{1}{2 p^{\prime}}=\frac{\theta}{2}+\frac{1-\theta}{q} .
$$

Aplicando o Lema $2.9 \operatorname{com} f:=\left.\widetilde{u}\right|_{B_{j}}$ obtemos

$$
|f|_{L^{2 p^{\prime}\left(B_{j}\right)}} \leq|f|_{L^{q}\left(B_{j}\right)}^{1-\theta}|f|_{L^{2}\left(B_{j}\right)}^{\theta}, \text { para todo } j \in \mathbb{N}
$$

Seja $\widetilde{\varepsilon}>0$. Assim,

$$
\begin{aligned}
|f|_{L^{2 p^{\prime}\left(B_{j}\right)}} & \leq|f|_{L^{q}\left(B_{j}\right)}^{1-\theta}|f|_{L^{2}\left(B_{j}\right)}^{\theta}=\frac{\left(\widetilde{\varepsilon}|f|_{L^{q}\left(B_{j}\right)}\right)^{1-\theta}}{\widetilde{\varepsilon}^{1-\theta}}|f|_{L^{2}\left(B_{j}\right)}^{\theta} \\
& =\left(\widetilde{\varepsilon}|f|_{L^{q}\left(B_{j}\right)}\right)^{1-\theta}\left(\widetilde{\varepsilon}^{\theta-1 / \theta}|f|_{L^{2}\left(B_{j}\right)}\right)^{\theta}
\end{aligned}
$$

Como

$$
\frac{1}{1 / \theta}+\frac{1}{1 /(1-\theta)}=1
$$

podemos usar a Desigualdade de Young (ver Lema 2.8) em (2.9) com $a=\left(\widetilde{\varepsilon}|f|_{q}\right)^{1-\theta}$ e $b=$ $\left(\widetilde{\varepsilon}^{\theta-1 / \theta}|f|_{2}\right)^{\theta}$ e segue que

$$
\begin{aligned}
|f|_{L^{2} p^{\prime}\left(B_{j}\right)} & \leq \frac{\left[\left(\widetilde{\varepsilon}|f|_{L^{q}\left(B_{j}\right)}\right)^{1-\theta}\right]^{1 / 1-\theta}}{1 / 1-\theta}+\frac{\left[\left(\widetilde{\varepsilon}^{\theta-1 / \theta}|f|_{L^{2}\left(B_{j}\right)}\right)^{\theta}\right]^{1 / \theta}}{1 / \theta} \\
& \leq(1-\theta) \widetilde{\varepsilon}|f|_{L^{q}\left(B_{j}\right)}+\theta \widetilde{\varepsilon}^{\theta-1 / \theta}|f|_{L^{2}\left(B_{j}\right)} \leq \widetilde{\varepsilon}|f|_{L^{q}\left(B_{j}\right)}+\theta \widetilde{\varepsilon}^{\theta-1 / \theta}|f|_{L^{2}\left(B_{j}\right)} \\
& \leq \widetilde{\varepsilon}|f|_{L^{q}\left(B_{j}\right)}+C_{\widetilde{\varepsilon}}|f|_{L^{2}\left(B_{j}\right)} \leq \widetilde{\varepsilon} M^{\prime}|f|_{H^{1}\left(B_{j}\right)}+C_{\widetilde{\varepsilon}}|f|_{L^{2}\left(B_{j}\right)},
\end{aligned}
$$

onde $C_{\widetilde{\varepsilon}}=\theta \widetilde{\varepsilon}^{\theta-1 / \theta}$. Portanto, a afirmativa está demonstrada.

Dado $\bar{\varepsilon} \in(0, \infty)$, defina $\widetilde{\varepsilon}=\left(M^{\prime}\right)^{-1}\left(\bar{\varepsilon} /\left(2|\beta|_{L_{u}^{p}\left(\mathbb{R}^{N}\right)}\right)\right)^{1 / 2}$. Logo, para todo $u \in H_{0}^{1}(\Omega)$ e para 
todo $j \in \mathbb{N}$ temos

$$
\left(\int_{B_{j}}|\widetilde{u}(x)|^{2 p^{\prime}} d x\right)^{1 / p^{\prime}} \leq 2\left(\widetilde{\varepsilon} M^{\prime}\right)^{2} \int_{B_{j}}\left(|\nabla \widetilde{u}(x)|^{2}+|\widetilde{u}(x)|^{2}\right) d x+2 C_{\widetilde{\varepsilon}}^{2} \int_{B_{j}}|\widetilde{u}(x)|^{2} d x
$$

e finalmente obtemos

$$
\begin{aligned}
\int_{\Omega}\left|\beta(x) u^{2}(x)\right| & d x \leq|\widetilde{\beta}|_{L_{u}^{p}\left(\mathbb{R}^{N}\right)} \sum_{j \in \mathbb{N}}\left(\int_{B_{j}}|\widetilde{u}(x)|^{2 p^{\prime}} d x\right)^{1 / p^{\prime}} \\
\leq & |\widetilde{\beta}|_{L_{u}^{p}\left(\mathbb{R}^{N}\right)} \sum_{j \in \mathbb{N}}\left(2\left(\widetilde{\varepsilon} M^{\prime}\right)^{2} \int_{B_{j}}\left(|\nabla \widetilde{u}(x)|^{2}+|\widetilde{u}(x)|^{2}\right) d x+2 C_{\widetilde{\varepsilon}}^{2} \int_{B_{j}}|\widetilde{u}(x)|^{2} d x\right) \\
& =|\beta|_{L_{u}^{p}\left(\mathbb{R}^{N}\right)} 2\left(\widetilde{\varepsilon} M^{\prime}\right)^{2}|u|_{H^{1}(\Omega)}^{2}+|\beta|_{L_{u}^{p}\left(\mathbb{R}^{N}\right)} 2 C_{\widetilde{\varepsilon}}^{2}|u|_{L^{2}(\Omega)}^{2} \\
& =\bar{\varepsilon}|u|_{H^{1}(\Omega)}^{2}+|\beta|_{L_{u}^{p}\left(\mathbb{R}^{N}\right)} 2 C_{\widetilde{\varepsilon}}^{2}|u|_{L^{2}(\Omega)}^{2} .
\end{aligned}
$$

Notemos que a demonstração está completa ao definirmos $C_{\bar{\varepsilon}}:=|\beta|_{L_{u}^{p}\left(\mathbb{R}^{N}\right)} 2 C_{\widetilde{\varepsilon}}^{2}$.

Iniciamos agora a definição do $C_{0}$-semigrupo associado à solução da equação da onda amortecida. As condições da Hipótese 2.5 (a) implicam que

$$
\sum_{i, j=1}^{N} \partial_{j}\left(a_{i j} \partial_{i} u\right) \in \mathscr{D}^{\prime}(\Omega), \text { para todo } u \in H_{0}^{1}(\Omega)
$$

Logo temos bem definido operador $L: H_{0}^{1}(\Omega) \rightarrow \mathscr{D}^{\prime}(\Omega)$ dado por

$$
L u=\sum_{i, j=1}^{N} \partial_{j}\left(a_{i j} \partial_{i} u\right), \quad u \in H_{0}^{1}(\Omega)
$$

A definição de derivada no sentido de distribuições implica que

$$
(L u-\beta u)(v)=-\langle A \nabla u, \nabla v\rangle-\langle\beta u, v\rangle, u \in H_{0}^{1}(\Omega), v \in \mathscr{D}(\Omega) .
$$

A densidade de $H_{0}^{1}(\Omega)$ em $L^{2}(\Omega)$ e a densidade de $\mathscr{D}(\Omega)$ em $H_{0}^{1}(\Omega)$ implicam que

$$
\langle(L u-\beta u), v\rangle=-\langle A \nabla u, \nabla v\rangle-\langle\beta u, v\rangle
$$

para todo $u, v \in H_{0}^{1}(\Omega) \operatorname{com} L u-\beta u \in L^{2}(\Omega)$.

Vamos introduzir em $H_{0}^{1}(\Omega)$ um novo produto interno. O próximo lema nos auxiliará em sua definição.

Lema 2.10. Assuma as condições da Hipótese 2.5 e seja $\kappa \in\left[0, \lambda_{1}\right)$ arbitrário. Sejam $\bar{\varepsilon} \in$ 
$\left(0, a_{0}\right)$ e $\rho \in(0,1)$ tais que $c:=\min \left\{\rho\left(a_{0}-\bar{\varepsilon}\right),(1-\rho)\left(\lambda_{1}-\kappa\right)-\rho\left(\bar{\varepsilon}+C_{\bar{\varepsilon}}+\kappa\right)\right\}>0$ e defina $C:=\max \left\{a_{1}+\bar{\varepsilon}, \bar{\varepsilon}+C_{\bar{\varepsilon}}\right\}$. Para todo $u \in H_{0}^{1}(\Omega)$,

$$
c\left(|\nabla u|_{L^{2}(\Omega)}^{2}+|u|_{L^{2}(\Omega)}^{2}\right) \leq\langle A \nabla u, \nabla u\rangle+\langle\beta u, u\rangle-\kappa\langle u, u\rangle \leq C\left(|\nabla u|_{L^{2}(\Omega)}^{2}+|u|_{L^{2}(\Omega)}^{2}\right) .
$$

Antes de demonstrar o lema acima, mostremos que existe uma constante $c$ nas condições deste lema. De fato, seja $\kappa \in\left[0, \lambda_{1}\right)$ e seja $\bar{\varepsilon} \in\left(0, a_{0}\right)$. Como $\lambda_{1}-\kappa>0$ e $\kappa \geq 0$, temos

$$
0<\frac{\lambda_{1}-\kappa}{\left(\lambda_{1}-\kappa\right)+\left(\bar{\varepsilon}+C_{\bar{\varepsilon}}+\kappa\right)}<1
$$

Seja $\rho \in \mathbb{R}$ tal que

$$
0<\rho<\frac{\lambda_{1}-\kappa}{\left(\lambda_{1}-\kappa\right)+\left(\bar{\varepsilon}+C_{\bar{\varepsilon}}+\kappa\right)}<1
$$

Segue que $\rho \in(0,1)$ e

$$
\left(\frac{1}{\rho}-1\right)\left(\lambda_{1}-\kappa\right)>\left(\bar{\varepsilon}+C_{\bar{\varepsilon}}+\kappa\right) .
$$

Como $a_{0}-\bar{\varepsilon}>0$ e $(1-\rho)\left(\lambda_{1}-\kappa\right)-\rho\left(\bar{\varepsilon}+C_{\bar{\varepsilon}}+\kappa\right)>0$ temos que $c>0$.

Demonstração do Lema 2.10 A definição de $\lambda_{1}$ implica que para todo $u \in H_{0}^{1}$ temos

$$
\lambda_{1}|u|_{L^{2}(\Omega)}^{2} \leq \int_{\Omega}\left[\sum_{i, j=1}^{N} a_{i j} \partial_{i} u \partial_{j} u+\beta|u|^{2}\right] d x
$$

e obtemos

$$
\begin{aligned}
& \int_{\Omega}\left(\sum_{i, j=1}^{N} a_{i j} \partial_{i} u \partial_{j} u+(\beta-\kappa)|u|^{2}\right) d x=\rho \int_{\Omega}\left(\sum_{i, j=1}^{N} a_{i j} \partial_{i} u \partial_{j} u+(\beta-\kappa)|u|^{2}\right) d x \\
& \quad+(1-\rho) \int_{\Omega}\left[\sum_{i, j=1}^{N} a_{i j} \partial_{i} u \partial_{j} u+(\beta-\kappa)|u|^{2}\right] d x \\
& \quad \geq \rho a_{0}|\nabla u|_{L^{2}(\Omega)}^{2}+\rho \int_{\Omega}(\beta-\kappa)|u|^{2} d x+(1-\rho) \lambda_{1}|u|_{L^{2}(\Omega)}^{2}-(1-\rho) \int_{\Omega} \kappa|u|^{2} d x \\
& \quad=\rho a_{0}|\nabla u|_{L^{2}(\Omega)}^{2}+(1-\rho)\left(\lambda_{1}-\kappa\right)|u|_{L^{2}(\Omega)}^{2}+\rho \int_{\Omega}(\beta-\kappa)|u|^{2} d x \\
& \quad=\rho a_{0}|\nabla u|_{L^{2}(\Omega)}^{2}+(1-\rho)\left(\lambda_{1}-\kappa\right)|u|_{L^{2}(\Omega)}^{2}+\rho \int_{\Omega} \beta|u|^{2}-\rho \int_{\Omega} \kappa|u|^{2} d x
\end{aligned}
$$


Usando a Proposição 2.7(b) na desigualdade acima, segue que

$$
\begin{aligned}
& \int_{\Omega}\left(\sum_{i, j=1}^{N} a_{i j} \partial_{i} u \partial_{j} u+(\beta-\kappa)|u|^{2}\right) d x \geq \rho a_{0}|\nabla u|_{L^{2}(\Omega)}^{2}+(1-\rho)\left(\lambda_{1}-\kappa\right)|u|_{L^{2}(\Omega)}^{2} \\
& -\rho\left(\bar{\varepsilon}|u|_{H^{1}(\Omega)}^{2}+C_{\bar{\varepsilon}}|u|_{L^{2}(\Omega)}^{2}\right)-\rho \kappa|u|_{L^{2}(\Omega)}^{2} \\
& =\rho a_{0}|\nabla u|_{L^{2}(\Omega)}^{2}+(1-\rho)\left(\lambda_{1}-\kappa\right)|u|_{L^{2}(\Omega)}^{2}-\rho\left[\bar{\varepsilon}\left(|u|_{L^{2}(\Omega)}^{2}+|\nabla u|_{L^{2}(\Omega)}^{2}\right)\right. \\
& \left.+C_{\bar{\varepsilon}}|u|_{L^{2}(\Omega)}^{2}\right)-\rho \kappa|u|_{L^{2}(\Omega)}^{2} \\
& =\rho\left(a_{0}-\bar{\varepsilon}\right)|\nabla u|_{L^{2}(\Omega)}^{2}+(1-\rho)\left(\lambda_{1}-\kappa\right)|u|_{L^{2}(\Omega)}^{2}-\rho\left(\bar{\varepsilon}+C_{\bar{\varepsilon}}+\kappa\right)|u|_{L^{2}(\Omega)}^{2} \\
& \geq c\left(|\nabla u|_{L^{2}(\Omega)}^{2}+|u|_{L^{2}(\Omega)}^{2}\right) \text {. }
\end{aligned}
$$

Por outro lado,

$$
\begin{gathered}
\int_{\Omega}\left(\sum_{i, j=1}^{N} a_{i j} \partial_{i} u \partial_{j} u+(\beta-\kappa)|u|^{2}\right) d x \leq \int_{\Omega} a_{1}|\nabla u|^{2} d x+\bar{\varepsilon}|u|_{H^{1}(\Omega)}^{2}+C_{\bar{\varepsilon}}|u|_{L^{2}(\Omega)}^{2}-\int_{\Omega} \kappa|u|^{2} d x \\
=\left(a_{1}+\bar{\varepsilon}\right)|\nabla u|_{L^{2}(\Omega)}^{2}+\left(\bar{\varepsilon}+C_{\bar{\varepsilon}}-\kappa\right)|u|_{L^{2}(\Omega)}^{2} \leq C\left(|\nabla u|_{L^{2}(\Omega)}^{2}+|u|_{L^{2}(\Omega)}^{2}\right)
\end{gathered}
$$

e a demonstração está concluída.

No que segue vamos assumir as condições da Hipótese 2.5. Seja $\varepsilon \in(0, \infty)$ arbitrário e defina

$$
\langle u, v\rangle_{1}=\frac{1}{\varepsilon}\langle A \nabla u, \nabla v\rangle+\frac{1}{\varepsilon}\langle\beta u, v\rangle, \text { para } u, v \in H_{0}^{1}(\Omega) .
$$

Lema 2.11. Para todos $u, v \in H_{0}^{1}(\Omega),\langle u, v\rangle_{1}$ define um número real. Além disso, a aplicação $(u, v) \in H_{0}^{1}(\Omega) \times H_{0}^{1}(\Omega) \mapsto\langle u, v\rangle_{1} \in \mathbb{R}$ define um produto escalar em $H_{0}^{1}(\Omega)$ e a norma definida por esse produto escalar é equivalente à norma usual de $H_{0}^{1}(\Omega)$.

Demonstração. Mostremos que para todo $u, v \in H_{0}^{1}(\Omega)$, a expressão $1 / \varepsilon\langle A \nabla u, \nabla v\rangle+$ $1 / \varepsilon\langle\beta u, v\rangle$ está bem definida. As condições da Hipótese 2.5 implicam que

$$
\langle\beta u, u\rangle=\int_{\Omega} \beta(x) u^{2}(x) d x<\infty, \text { para todo } u \in H_{0}^{1}(\Omega) .
$$

Assim, para $u, v \in H_{0}^{1}(\Omega)$ temos

$$
\begin{aligned}
\langle\beta u, v\rangle & \leq|\langle\beta u, v\rangle|=\left|\int_{\Omega} \beta(x) u(x) v(x) d x\right| \leq\left.\int_{\Omega}|| \beta(x)\right|^{1 / 2} u(x)|||\beta(x)|^{1 / 2} v(x) \mid d x \\
& \leq\left[\int_{\Omega} \beta(x) u^{2}(x) d x\right]^{1 / 2}\left[\int_{\Omega} \beta(x) v^{2}(x) d x\right]^{1 / 2}
\end{aligned}
$$


e, portanto $\langle\beta u, v\rangle$ está bem definido. Por outro lado, seguem das condições da Hipótese 2.5 que

$$
\langle A \nabla u, \nabla u\rangle \leq \int_{\Omega} a_{1}|\nabla u(x)|^{2} d x \leq a_{1}|u|_{H_{0}^{1}(\Omega)}, \text { para todo } u \in H_{0}^{1}(\Omega) . .
$$

Logo, para $u, v \in H_{0}^{1}(\Omega)$ temos

$$
\begin{aligned}
\langle A \nabla u, \nabla v\rangle & =\int_{\Omega} \sum_{i, j=1}^{N} a_{i j}(x) \partial_{i} u(x) \partial_{j} v(x) d x \\
& =\int_{\Omega} \sum_{i, j=1}^{N} \frac{a_{i j}(x)}{4}\left(\partial_{i} u(x)+\partial_{j} v(x)\right)^{2} d x-\int_{\Omega} \sum_{i, j=1}^{N} \frac{a_{i j}(x)}{4}\left(\partial_{i} u(x)-\partial_{j} v(x)\right)^{2} d x \\
& =\frac{1}{4}(\langle A \nabla(u+v), \nabla(u+v)\rangle-\langle A \nabla(u-v), \nabla(u-v)\rangle)
\end{aligned}
$$

e, novamente, temos que $\langle A \nabla u, \nabla v\rangle$ está bem definido. Afirmamos que a aplicação

$$
\langle u, v\rangle_{1}=\frac{1}{\varepsilon}\langle A \nabla u, \nabla v\rangle+\frac{1}{\varepsilon}\langle\beta u, v\rangle, \text { para } u, v \in H_{0}^{1}(\Omega),
$$

é um produto interno em $H_{0}^{1}(\Omega)$. Dado $u \in H_{0}^{1}(\Omega)$, segue do Lema 2.10, com $\kappa=0$, que

$$
c|u|_{H_{0}^{1}(\Omega)}^{2} \leq\langle u, u\rangle_{1}=\frac{1}{\varepsilon}\langle A \nabla u, \nabla u\rangle+\frac{1}{\varepsilon}\langle\beta u, u\rangle \leq C|u|_{H_{0}^{1}(\Omega)}^{2}
$$

Portanto, $\langle u, u\rangle_{1} \geq 0$ e $\langle u, u\rangle_{1}=0$ se, e somente, se $u=0$.

É simples mostrar que $\langle\lambda u+v, w\rangle_{1}=\lambda\langle u, v\rangle_{1}+\langle u, w\rangle_{1}$ e que $\langle u, v\rangle_{1}=\langle v, u\rangle_{1}$ para todo $u$, $v \in H_{0}^{1}(\Omega)$ e $\lambda \in \mathbb{R}$, o que conclui a demonstração da afirmação. Notemos que a desigualdade (2.12) implica que a norma definida por $\langle\cdot, \cdot\rangle_{1}$ em $H_{0}^{1}(\Omega)$ é equivalente à norma usual de $H_{0}^{1}(\Omega)$.

A norma em $H_{0}^{1}(\Omega)$ proveniente do produto interno $\langle\cdot, \cdot\rangle_{1}$ será denotada por $|\cdot|_{1}$.

Observação 2.12. $\mathscr{D}(\Omega)$ é um conjunto denso em $H_{0}^{1}(\Omega)$ munido da norma $|\cdot|_{1}$.

O lema a seguir relaciona os funcionais lineares de $H_{0}^{1}(\Omega)$ obtidos pelas normas equivalentes $|\cdot|_{H_{0}^{1}(\Omega)} \mathrm{e}|\cdot|_{1}$ de $H_{0}^{1}(\Omega)$.

Lema 2.13. Suponha a notação introduzida no Lema 2.11 Temos que $f \in H^{-1}(\Omega)$ se, e somente se, $f \in\left(H_{0}^{1}(\Omega),|\cdot|_{1}\right)^{\prime}$. Além disso,

$$
\frac{1}{c}|f|_{1} \leq|f| \leq \frac{C}{c}|f|_{1}, \text { para todo } f \in H^{-1}(\Omega),
$$

onde $|f|=\sup \left\{|f(x)|\left|u \in H_{0}^{1}(\Omega) e\right| u \mid \leq 1\right\} e|f|_{1}=\sup \left\{\left.|f(x)|\left|u \in H_{0}^{1}(\Omega) e\right| u\right|_{1} \leq 1\right\}$. 
Demonstração. A demonstração é uma consequência imediata da equivalência entre as normas $|\cdot|_{1} \mathrm{e}|\cdot|_{H_{0}^{1}(\Omega)}$ em $H_{0}^{1}(\Omega)$.

Lema 2.14. Para cada $u \in H_{0}^{1}(\Omega)$, a distribuição

$$
-(1 / \varepsilon) L u+(1 / \varepsilon) \beta u \in \mathscr{D}^{\prime}(\Omega)
$$

pode ser unicamente estendida a um funcional linear contínuo $f_{u}: H_{0}^{1}(\Omega) \rightarrow \mathbb{R}$. O operador $\Lambda: H_{0}^{1}(\Omega) \rightarrow H^{-1}(\Omega)=\left(H_{0}^{1}(\Omega)\right)^{\prime}$ dado por

$$
\Lambda(u)=f_{u}, \quad u \in H_{0}^{1}(\Omega)
$$

é um isomorfismo.

Demonstração. Seja $u \in H_{0}^{1}(\Omega)$ e considere a distribuição $(1 / \varepsilon) L u+(1 / \varepsilon) \beta u=: T_{u}$. Temos que

$$
T_{u}(v)=\frac{1}{\varepsilon}\langle A \nabla u, \nabla v\rangle+\frac{1}{\varepsilon}\langle\beta u, v\rangle=\langle u, v\rangle_{1}, \text { para } v \in \mathscr{D}(\Omega)
$$

Logo,

$$
\left|T_{u}(v)\right| \leq|u|_{1}|v|_{1} \leq C^{2}|u|_{H_{0}^{1}(\Omega)}|v|_{H_{0}^{1}(\Omega)} \text { para } u \in H_{0}^{1}(\Omega) \text { e } v \in \mathscr{D}(\Omega) .
$$

Segue que $T_{u}: \mathscr{D}(\Omega) \rightarrow \mathbb{R}$ é um funcional linear limitado. Como $\mathscr{D}(\Omega)$ é um subespaço vetorial de $H_{0}^{1}(\Omega)$ o Teorema de Hahn-Banach implica que existe um funcional linear limitado $f_{u}$ em $H^{-1}(\Omega)$ tal que $f_{u}$ estende $T_{u}$. Defina $\Lambda: H_{0}^{1}(\Omega) \rightarrow H^{-1}(\Omega)$ por $\Lambda(u)=f_{u}$ para $u \in H_{0}^{1}(\Omega)$. Logo, dado $u \in H_{0}^{1}(\Omega)$, segue do Lema 2.13 que $f_{u} \in\left(H_{0}^{1}(\Omega),|\cdot|_{1}\right)^{\prime}$. Uma aplicação do Teorema da Representação de Riesz e equação (2.13) implicam que

$$
f_{u}(v)=\langle u, v\rangle_{1}, \text { para todo } v \in H_{0}^{1}(\Omega)
$$

e $\left|f_{u}\right|=|u|_{1}$. Disso segue que $\Lambda$ é linear e injetora. Seja $f \in H^{-1}(\Omega)$, segue do Lema 2.13 que $f \in\left(H_{0}^{1}(\Omega),|\cdot|_{1}\right)^{\prime}$. Portanto, existe um $\widetilde{u} \in H_{0}^{1}(\Omega)$ tal que

$$
f(v)=\langle\widetilde{u}, v\rangle_{1}, \quad v \in H_{0}^{1}(\Omega)
$$

Portanto, $f_{\widetilde{u}}=f$.

Com a notação introduzida no Lema 2.14 temos que $\sup \left\{|\Lambda(u)| \mid u \in H_{0}^{1}(\Omega)\right.$ e $\left.|u|_{1} \leq 1\right\}=$ $|u|_{1}$. 
Lema 2.15. A aplicação

$$
(f, g) \in H^{-1}(\Omega) \times H^{-1}(\Omega) \mapsto\langle f, g\rangle_{-1}:=\left\langle\Lambda^{-1}(f), \Lambda^{-1}(g)\right\rangle_{1}
$$

define um produto interno em $H^{-1}(\Omega)$. A norma definida por esse produto interno é equivalente à norma usual em $H^{-1}(\Omega)$.

Demonstração. Seja $\Lambda$ como no Lema 2.14. Como $\Lambda$ é um isomorfismo, dado $f \in H^{-1}(\Omega)$, sabemos que existe um único $u \in H_{0}^{1}(\Omega)$ tal que $\Lambda^{-1}(f)=u$. Assim, as propriedades de $\langle\cdot, \cdot\rangle_{-1}$ são herdadas de $\langle\cdot, \cdot\rangle_{1}$ e isso implica que $\langle\cdot, \cdot\rangle_{-1}$ define um produto interno em $H^{-1}(\Omega)$.

Seja $f \in H^{-1}(\Omega)$ e seja $u \in H_{0}^{1}(\Omega)$ tal que $\Lambda^{-1}(f)=u$. Temos que

$$
|f|_{-1}^{2}=\langle f, f\rangle_{-1}=\langle u, u\rangle_{1}=|u|_{1}^{2} .
$$

Por outro lado, segue do Lema 2.13 que

$$
\frac{1}{c}|f|_{1} \leq|f| \leq \frac{C}{c}|f|_{1}
$$

Como $f(v)=\langle u, v\rangle_{1}$, para todo $v \in H_{0}^{1}(\Omega)$, segue que

$$
|f|_{1}=|u|_{1}
$$

Logo, 2.15), 2.16) e 2.17) implicam que

$$
\frac{1}{c}|f|_{1} \leq|f| \leq \frac{C}{c}|f|_{1}
$$

e o lema está demonstrado.

No que segue, considere também as seguintes hipóteses adicionais:

Hipótese 2.16. (a) $\alpha_{0}, \alpha_{1} \in[0, \infty)$.

(b) $\alpha: \Omega \rightarrow \mathbb{R}$ é uma função mensurável com $\alpha_{0} \leq \alpha(x) \leq \alpha_{1}$ para quase todo $x$ em $\Omega$.

(c) $Z:=H_{0}^{1}(\Omega) \times L^{2}(\Omega)$ munido da norma $|\cdot|_{Z}$ dada por

$$
|z|_{Z}^{2}=\left|\nabla z_{1}\right|_{L^{2}(\Omega)}^{2}+\left|z_{1}\right|_{L^{2}(\Omega)}^{2}+\left|z_{2}\right|_{L^{2}(\Omega)}^{2}, \operatorname{para} z=\left(z_{1}, z_{2}\right) \in Z
$$

(d) $\varepsilon \in(0, \infty)$. 
(e) $D(B)=D\left(B_{\alpha, \beta, \varepsilon}\right)$ é o conjunto de pontos $z=\left(z_{1}, z_{2}\right) \in Z$ tais que $z_{2} \in H_{0}^{1}(\Omega)$ e $L z_{1}-\beta z_{1}$ (no sentido de distribuições) está em $L^{2}(\Omega)$.

(f) $B=B_{\alpha, \beta, \varepsilon}: D(B) \rightarrow Z$ é o operador linear dado por

$$
B\left(z_{1}, z_{2}\right)=\left(z_{2},-(1 / \varepsilon) \alpha z_{2}+(1 / \varepsilon)\left(L z_{1}-\beta z_{1}\right), \text { para }\left(z_{1}, z_{2}\right) \in D(B) .\right.
$$

Proposição 2.17. Assuma as condições da Hipótese 2.5e da Hipótese 2.16 O operador B é o gerador infinitesimal de um $C_{0}$-semigrupo $T(t)=T_{\alpha, \beta, \varepsilon}(t), t \in[0, \infty)$, em Z. Se $\alpha_{0}>0$, então existem constantes $M=M\left(\alpha_{0}, \alpha_{1}, \varepsilon, \lambda_{1}\right)>0$ e $\mu=\mu\left(\alpha_{0}, \alpha_{1}, \varepsilon, \lambda_{1}\right)>0$ tais que

$$
|T(t)|_{Z} \leq M e^{-\mu t}|z|_{Z}, z \in Z \text { e } t \in[0, \infty)
$$

Demonstração. Considere em $Z$ o produto interno dado por

$$
\left\langle\left\langle\left(u_{1}, u_{2}\right),\left(w_{1}, w_{2}\right)\right\rangle\right\rangle=\left\langle u_{1}, w_{1}\right\rangle_{1}+\left\langle u_{2}, w_{2}\right\rangle_{L^{2}(\Omega)},\left(u_{1}, u_{2}\right),\left(w_{1}, w_{2}\right) \in Z .
$$

e denote por $\|\cdot\|$ a norma em $Z$ proveniente desse produto interno. Note que a norma $\|\cdot\|$ é equivalente à norma $|\cdot| z$. De fato, defina $k_{1}=\min \{1, c / \varepsilon\}$ e $k_{2}=\max \{1, C / \varepsilon\}$, onde as constantes $c$ e $C$ são como no Lema 2.10. Segue do Lema 2.10 com $\kappa=0$ que

$$
\begin{aligned}
k_{1}\left|\left(u_{1}, u_{2}\right)\right|_{Z}^{2} & =k_{1}\left(\left|u_{1}\right|_{H_{0}^{1}(\Omega)}^{2}+\left|u_{2}\right|_{L^{2}(\Omega)}^{2}\right) \leq(c / \varepsilon)\left|u_{1}\right|_{H_{0}^{1}(\Omega)}^{2}+\left|u_{2}\right|_{L^{2}(\Omega)}^{2} \\
& \leq\left\langle u_{1}, u_{1}\right\rangle_{1}+\left\langle u_{2}, u_{2}\right\rangle_{L^{2}(\Omega)}=\|\left.\left(u_{1}, u_{2}\right)\right|^{2} \leq(C / \varepsilon)\left|u_{1}\right|_{H_{0}^{1}(\Omega)}^{2}+\left|u_{2}\right|_{L^{2}(\Omega)}^{2} \\
& \leq k_{2}\left(\left|u_{1}\right|_{H_{0}^{1}(\Omega)}^{2}+\left|u_{2}\right|_{L^{2}(\Omega)}^{2}\right)=k_{2}\left|\left(u_{1}, u_{2}\right)\right|_{Z}^{2} .
\end{aligned}
$$

Logo, as normas são equivalentes. Portanto, $(Z,\langle\langle\cdot, \cdot\rangle\rangle)$ é um espaço de Hilbert.

Afirmamos que $B$ é um operador dissipativo. Seja $\left(z_{1}, z_{2}\right) \in D(B)$. Usando a expressão 2.11) obtemos

$$
\begin{aligned}
\left\langle\left\langle B\left(z_{1}, z_{2}\right),\left(z_{1}, z_{2}\right)\right\rangle\right\rangle & =\left\langle z_{2}, z_{1}\right\rangle{ }_{1}+\left\langle-(1 / \varepsilon) \alpha z_{2}+(1 / \varepsilon)\left(L z_{1}-\beta z_{1}\right), z_{2}\right\rangle \\
& =(1 / \varepsilon)\left\langle A \nabla z_{2}, \nabla z_{1}\right\rangle+(1 / \varepsilon)\left\langle\beta z_{2}, z_{1}\right\rangle-(1 / \varepsilon)\left\langle\alpha z_{2}, z_{2}\right\rangle \\
& -(1 / \varepsilon)\left\langle A \nabla z_{1}, \nabla z_{2}\right\rangle-(1 / \varepsilon)\left\langle\beta z_{1}, z_{2}\right\rangle \\
& =-(1 / \varepsilon)\left\langle\alpha z_{2}, z_{2}\right\rangle .
\end{aligned}
$$

Logo, Proposição 1.38 implica a afirmativa. 
Mostremos agora que para todo $\lambda>0$ e para todo $(f, g) \in Z$, existe um $\left(z_{1}, z_{2}\right) \in D(B)$ com

$$
\left(z_{1}, z_{2}\right)-\lambda B\left(z_{1}, z_{2}\right)=(f, g)
$$

Ou seja, devemos mostrar que para todo $\lambda>0$ vale $R(I-\lambda B)=Z$. Notemos que a igualdade (2.20) é equivalente a mostrar que para todo $\lambda>0$ e para todo $(f, g) \in Z$ existe um $\left(z_{1}, z_{2}\right) \in D(B)$ tal que

$$
z_{2}=\frac{1}{\lambda}\left(z_{1}-f\right)
$$

$\mathrm{e}$

$$
\left(\frac{1}{\lambda}+\frac{1}{\varepsilon} \alpha\right) z_{1}-\frac{\lambda}{\varepsilon}\left(L z_{1}+\beta z_{1}\right)=g+\left(\frac{1}{\lambda}+\frac{1}{\varepsilon} \alpha\right) f
$$

Mostremos que existe um $z_{1} \in H_{0}^{1}$ tal que a igualdade (2.22) é verificada. Considere a forma bilinear definida por

$$
b(u, v)=\frac{\lambda}{\varepsilon}(\langle A \nabla u, \nabla v\rangle+\langle\beta u, v\rangle)+\frac{1}{\lambda}\langle u, v\rangle+\frac{1}{\varepsilon}\langle\alpha u, v\rangle, \text { para } u, v \in H_{0}^{1}(\Omega) .
$$

Mostremos que a forma bilinear $b$ é contínua e coersiva. De fato, notemos que

$$
\begin{aligned}
|b(u, v)| & \leq \frac{\lambda}{\varepsilon}|\langle A \nabla u, \nabla v\rangle+\langle\beta u, v\rangle|+\frac{1}{\lambda}|\langle u, v\rangle|+\frac{1}{\varepsilon}|\langle\alpha u, v\rangle| \\
& \leq \lambda\left|\langle u, v\rangle_{1}\right|+\left(\frac{\alpha_{1}}{\varepsilon}+\frac{1}{\lambda}\right)\left|\langle u, v\rangle_{L^{2}(\Omega)}\right| \\
& \leq \lambda|u|_{H_{0}^{1}(\Omega)}|v|_{H_{0}^{1}(\Omega)}+\left(\frac{\alpha_{1}}{\varepsilon}+\frac{1}{\lambda}\right)|u|_{L^{2}(\Omega)}|v|_{L^{2}(\Omega)} \\
& \leq \lambda|u|_{H_{0}^{1}(\Omega)}|v|_{H_{0}^{1}(\Omega)}+\left(\frac{\alpha_{1}}{\varepsilon}+\frac{1}{\lambda}\right)|u|_{H_{0}^{1}(\Omega)}|v|_{H_{0}^{1}(\Omega)} \\
& \leq K|u|_{H_{0}^{1}(\Omega)}|v|_{H_{0}^{1}(\Omega)},
\end{aligned}
$$

onde $K:=\max \left[\left(\frac{\alpha_{1}}{\varepsilon}+\frac{1}{\lambda}\right), \lambda\right]$. Logo, $b$ é contínua. Para mostrar a coersividade de $b$, notemos que

$$
\begin{aligned}
|b(u, u)| & =\left|\frac{\lambda}{\varepsilon}(\langle A \nabla u, \nabla u\rangle+\langle\beta u, u\rangle)+\frac{1}{\lambda}\langle u, u\rangle+\frac{1}{\varepsilon}\langle\alpha u, u\rangle\right| \\
& \geq \frac{\lambda \lambda_{1}}{\varepsilon}|u|_{H_{0}^{1}(\Omega)}^{2}+\left(\frac{\alpha_{0}}{\varepsilon}+\frac{1}{\lambda}\right)|u|_{L^{2}(\Omega)}^{2} \\
& \geq \delta|u|_{H_{0}^{1}(\Omega)}^{2},
\end{aligned}
$$

onde $\delta=\frac{1}{\varepsilon} \lambda_{1} \lambda$. Segue do Teorema de Lax-Milgram [8, Teorema 1.1.4] que para todo $h \in$ 
$L^{2}(\Omega)$ existe um $z \in H_{0}^{1}(\Omega)$ tal que

$$
b(z, v)=\int_{\Omega} h(x) v(x) d x, \quad \text { para todo } v \in H_{0}^{1}(\Omega) .
$$

Portanto, $-(1 / \varepsilon) \lambda L z+(1 / \varepsilon) \lambda \beta z+(1 / \lambda) z+(1 / \varepsilon) \alpha z=h$ no sentido de distribuições. Assim,

$$
h-\frac{1}{\lambda} z-\frac{1}{\varepsilon} \alpha z \in L^{2}(\Omega)
$$

e, portanto, temos que $-(1 / \varepsilon) \lambda L z+(1 / \varepsilon) \lambda \beta z \in L^{2}(\Omega)$ no sentido de distribuições. Agora considere

$$
h=g+\left(\frac{1}{\lambda}+\frac{1}{\varepsilon} \alpha\right) f .
$$

Utilizando igualdade (2.23) encontramos um $z_{1} \in H_{0}^{1}(\Omega)$ satisfazendo 2.22 tal que $L z_{1}-\beta z_{1} \in$ $L^{2}(\Omega)$ no sentido de distribuições.

Uma vez obtido $z_{1}$, é fácil ver que existe um $z_{2} \in H_{0}^{1}(\Omega)$ tal que equação 2.21) é satisfeita. Em particular, $R(I-B)=X$ e segue do Teorema 1.37 que $D(B)$ é denso em $Z$. Portanto, pelo Teorema 1.34, temos que $B$ gera um $C_{0}$-semigrupo $\{T(t) \mid t \geq 0\}$ em $Z$.

Suponha agora que $\alpha_{0}>0$ e escolha $\mu \in \mathbb{R}$ tal que

$$
0<2 \mu \leq \min \left(1, \frac{\alpha_{0}}{2 \varepsilon}, \frac{\lambda_{1}}{\left(\varepsilon+\alpha_{1}\right)}\right)
$$

Afirmamos que

$$
\left\|T(t)\left(u_{1}, u_{2}\right)\right\| \leq 2 e^{-\mu t}\left\|\left(u_{1}, u_{2}\right)\right\|, \text { para todo } t \in[0, \infty) \text { e }\left(u_{1}, u_{2}\right) \in Z
$$

De fato, consideremos o caso em que $\left(u_{1}, u_{2}\right) \in D(B)$ e defina

$$
\left(z_{1}(t), z_{2}(t)\right):=T(t)\left(u_{1}, u_{2}\right), t \in[0, \infty)
$$

A Proposição 1.26 implica que a aplicação $t \in[0, \infty) \mapsto z(t)=\left(z_{1}(t), z_{2}(t)\right) \in Z$ é diferenciável, $z(t) \in D(B)$ e $z^{\prime}(t)=B z(t)$, para todo $t \in[0, \infty)$. Para $t \in[0, \infty)$, defina a aplicação

$$
\begin{aligned}
w(t) & =4 \mu\left\langle z_{1}(t), z_{2}(t)\right\rangle+\left\langle z_{2}(t), z_{2}(t)\right\rangle+2 \frac{1}{\varepsilon} \mu\left\langle\alpha z_{1}(t), z_{1}(t)\right\rangle \\
& +\frac{1}{\varepsilon}\left\langle\beta z_{1}(t), z_{1}(t)\right\rangle+\frac{1}{\varepsilon}\left\langle A \nabla z_{1}(t), \nabla z_{1}(t)\right\rangle .
\end{aligned}
$$


Temos que $w$ é diferenciável e

$$
\begin{aligned}
\frac{1}{2} w^{\prime}(t) & =\left\langle(2 \mu-(1 / \varepsilon) \alpha) z_{2}(t), z_{2}(t)\right\rangle-2 \mu(1 / \varepsilon)\left\langle\beta z_{1}(t), z_{1}(t)\right\rangle \\
& -2(1 / \varepsilon) \mu\left\langle A \nabla z_{1}(t), \nabla z_{1}(t)\right\rangle
\end{aligned}
$$

A Hipótese 2.16(b) implica que

$$
\begin{aligned}
\frac{1}{2} w^{\prime}(t) & \leq\left\langle\left(2 \mu-(1 / \varepsilon) \alpha_{0}\right) z_{2}(t), z_{2}(t)\right\rangle-2 \mu(1 / \varepsilon)\left\langle\beta z_{1}(t), z_{1}(t)\right\rangle \\
& -2(1 / \varepsilon) \mu\left\langle A \nabla z_{1}(t), \nabla z_{1}(t)\right\rangle
\end{aligned}
$$

Como $2\left\langle z_{1}(t), z_{2}(t)\right\rangle \leq\left\langle z_{1}(t), z_{1}(t)\right\rangle+\left\langle z_{2}(t), z_{2}(t)\right\rangle$ para todo $t \geq 0$, segue de (2.26) que

$$
\begin{aligned}
w(t) & \leq 4 \mu\left(\frac{1}{2}\left\langle z_{1}(t), z_{1}(t)\right\rangle+\frac{1}{2}\left\langle z_{2}(t), z_{2}(t)\right\rangle\right)+\left\langle z_{2}(t), z_{2}(t)\right\rangle \\
& +2 \frac{1}{\varepsilon} \mu\left\langle\alpha z_{1}(t), z_{1}(t)\right\rangle+\frac{1}{\varepsilon}\left\langle\beta z_{1}(t), z_{1}(t)\right\rangle+\frac{1}{\varepsilon}\left\langle A \nabla z_{1}(t), \nabla z_{1}(t)\right\rangle \\
& \leq(2 \mu+1)\left\langle z_{2}(t), z_{2}(t)\right\rangle+2 \mu\left(1+\frac{1}{\varepsilon} \alpha_{1}\right)\left\langle z_{1}(t), z_{1}(t)\right\rangle \\
& +\frac{1}{\varepsilon}\left\langle\beta z_{1}(t), z_{1}(t)\right\rangle+\frac{1}{\varepsilon}\left\langle A \nabla z_{1}(t), \nabla z_{1}(t)\right\rangle .
\end{aligned}
$$

Notemos que

$$
2\|z(t)\|^{2}=2\left\langle z_{2}(t), z_{2}(t)\right\rangle+2(1 / \varepsilon)\left\langle\beta z_{1}(t), z_{1}(t)\right\rangle+2(1 / \varepsilon)\left\langle A \nabla z_{1}(t), \nabla z_{1}(t)\right\rangle .
$$

A condição imposta à escolha da constante $\mu$ em (2.24) implica que, para todo $t \geq 0$,

$$
2\left\langle z_{2}(t), z_{2}(t)\right\rangle \geq(2 \mu+1)\left\langle z_{2}(t), z_{2}(t)\right\rangle
$$

e

$$
\begin{aligned}
\frac{1}{\varepsilon}\left\langle\beta z_{1}(t), z_{1}(t)\right\rangle+\frac{1}{\varepsilon}\left\langle A \nabla z_{1}(t), \nabla z_{1}(t)\right\rangle & \geq \frac{1}{\varepsilon} \lambda_{1}\left\langle z_{1}(t), z_{1}(t)\right\rangle \\
& \geq 2 \mu\left(1+\frac{1}{\varepsilon} \alpha_{1}\right)\left\langle z_{1}(t), z_{1}(t)\right\rangle .
\end{aligned}
$$

Portanto obtemos

$$
w(t) \leq 2\|z(t)\|^{2}, \text { para todo } t \in[0, \infty) .
$$


Por outro lado, utilizando novamente a condição descrita em (2.24) obtemos

$$
\begin{aligned}
w(t) \geq & -4 \mu\left(\frac{1}{2} 4 \mu\left\langle z_{1}(t), z_{1}(t)\right\rangle+\frac{1}{2} \frac{1}{4 \mu}\left\langle z_{2}(t), z_{2}(t)\right\rangle\right)+\left\langle z_{2}(t), z_{2}(t)\right\rangle \\
& +2 \frac{1}{\varepsilon} \mu\left\langle\alpha z_{1}(t), z_{1}(t)\right\rangle+\frac{1}{\varepsilon}\left\langle\beta z_{1}(t), z_{1}(t)\right\rangle+\frac{1}{\varepsilon}\left\langle A \nabla z_{1}(t), \nabla z_{1}(t)\right\rangle \\
& \geq \frac{1}{2}\left\langle z_{2}(t), z_{2}(t)\right\rangle+2 \mu\left(\frac{1}{\varepsilon} \alpha_{0}-4 \mu\right)\left\langle z_{1}(t), z_{1}(t)\right\rangle+\frac{1}{\varepsilon}\left\langle\beta z_{1}(t), z_{1}(t)\right\rangle \\
& +\frac{1}{\varepsilon}\left\langle A \nabla z_{1}(t), \nabla z_{1}(t)\right\rangle \geq \frac{1}{2}\|z(t)\|^{2}
\end{aligned}
$$

ou seja,

$$
w(t) \geq \frac{1}{2}\|z(t)\|^{2}, \text { para todo } t \in[0, \infty) .
$$

As desigualdades 2.28), 2.27) e 2.24) implicam que

$$
\begin{aligned}
\mu w(t) \leq 2 \mu\|z(t)\|^{2} & =2 \mu\left\langle z_{2}(t), z_{2}(t)\right\rangle+2 \mu \frac{1}{\varepsilon}\left\langle\beta z_{1}(t), z_{1}(t)\right\rangle+2 \mu \frac{1}{\varepsilon}\left\langle A \nabla z_{1}(t), \nabla z_{1}(t)\right\rangle \\
& \leq\left(\frac{1}{\varepsilon} \alpha_{0}-2 \mu\right)\left\langle z_{2}(t), z_{2}(t)\right\rangle+2 \mu \frac{1}{\varepsilon}\left\langle\beta z_{1}(t), z_{1}(t)\right\rangle \\
& +2 \mu \frac{1}{\varepsilon}\left\langle A \nabla z_{1}(t), \nabla z_{1}(t)\right\rangle \leq-\frac{1}{2} w^{\prime}(t) .
\end{aligned}
$$

Aqui usamos que $2 \mu \leq(1 / \varepsilon) \alpha_{0}-2 \mu$. Portanto

$$
w^{\prime}(t) \leq-2 \mu w(t), \text { para todo } t \in[0, \infty)
$$

Assim, $\frac{d}{d t}\left(w(t) e^{2 \mu t}\right) \leq 0$ para todo $t \in[0, \infty)$ e obtemos que

$$
w(t) e^{2 \mu t}-w(0) \leq 0, \text { para todo } t \in[0, \infty)
$$

Logo,

$$
w(t) \leq w(0) e^{-2 \mu t}, \text { para todo } t \in[0, \infty)
$$

A desigualdade (2.28) implica que $w(0) \leq 2\|z(0)\|^{2}$. Finalmente, utilizando as informações obtidas juntamente com a desigualdade (2.29) concluimos que

$$
\|z(t)\|^{2} \leq 2 w(t) \leq 2 w(0) e^{-2 \mu t} \leq 4 e^{-2 \mu t}\|z(0)\|^{2}, \text { para todo } t \in[0, \infty)
$$

Portanto, a relação em (2.25) é satisfeita para $\left(u_{1}, u_{2}\right) \in D(B)$. A densidade do conjunto $D(B)$ em $Z$ implica que a desigualdade 2.25) também é válida para $\left(u_{1}, u_{2}\right) \in Z$. Para concluir a 
demonstração, notemos que para todo $t \in[0, \infty)$ e $\left(u_{1}, u_{2}\right) \in Z$ temos

$$
k_{1}\left|T(t)\left(u_{1}, u_{2}\right)\right|_{Z}^{2} \leq\left\|T(t)\left(u_{1}, u_{2}\right)\right\|^{2} \leq 4 e^{-2 \mu t}\left\|\left(u_{1}, u_{2}\right)\right\|^{2} \leq 4 e^{-2 \mu t} k_{2}\left|\left(u_{1}, u_{2}\right)\right|_{Z}^{2} .
$$

Defina $M=2 \sqrt{k_{2} / k_{1}}$. Finalmente obtemos

$$
\left|T(t)\left(u_{1}, u_{2}\right)\right|_{Z} \leq M e^{-\mu t}\left|\left(u_{1}, u_{2}\right)\right|_{Z},\left(u_{1}, u_{2}\right) \in Z \text { e } t \in[0, \infty) .
$$

A demonstração está completa.

Concluímos este capítulo com dois resultados que serão utilizados para mostrar a existência de atrator global para o caso crítico.

Defina $Y=L^{2}(\Omega) \times H^{-1}(\Omega)$ e seja $v: Z \rightarrow Y$ a aplicação inclusão dada por $v(u, w)=$ $\left(u, \varphi_{w}\right),(u, w) \in Z$, onde, para $w \in L^{2}(\Omega), \varphi_{w}: H^{-1}(\Omega) \rightarrow \mathbb{R}$ é definida por $\varphi_{w}(z)=\langle z, w\rangle_{L^{2}(\Omega)}$, $z \in H_{0}^{1}(\Omega)$. Observamos que $v$ é uma aplicação injetiva.

Proposição 2.18. Assuma as condições da Hipótese 2.5 e da Hipótese 2.16 Defina $C_{Z}:=B_{\alpha, \beta, \varepsilon}$ $e S_{Z}(t)=T_{\alpha, \beta, \varepsilon}(t)$, para $t \in[0, \infty)$ e com $\alpha \equiv 0$. Além disso, defina $D\left(C_{Y}\right)=v\left(H_{0}^{1}(\Omega) \times L^{2}(\Omega)\right)$ e considere o operador linear $C_{Y}: D\left(C_{Y}\right) \rightarrow Y$ por

$$
C_{Y}\left(y_{1}, y_{2}\right)=\left(z_{2},-\Lambda\left(y_{1}\right)\right), \text { para }\left(y_{1}, y_{2}\right) \in D\left(C_{Y}\right)
$$

onde $z_{2} \in L^{2}(\Omega)$ é tal que $v\left(y_{1}, z_{2}\right)=\left(y_{1}, y_{2}\right)$ e $\Lambda$ é como definido no Lema 2.14. Então $C_{Y}$ é gerador de um $C_{0}$-semigrupo $\left\{S_{Y}(t) \mid t \geq 0\right\}$, e

$$
v\left(S_{Z}(t) z\right)=S_{Y}(t)(v(z)), \text { para todo } z \in Z \text { e } t \in[0, \infty),
$$

Demonstração. Considere em $Y$ o produto interno dado por

$$
\left\langle\left\langle\left(u_{1}, u_{2}\right),\left(w_{1}, w_{2}\right)\right\rangle\right\rangle=\left\langle u_{1}, w_{1}\right\rangle_{L^{2}(\Omega)}+\left\langle u_{2}, w_{2}\right\rangle_{-1} \text {, para }\left(u_{1}, u_{2}\right),\left(w_{1}, w_{2}\right) \in Y .
$$

Segue do Lema 2.15 que a norma definida por esse produto escalar é equivalente à norma usual em $Y$. Afirmamos que

$$
\left\langle\left\langle C_{Y}\left(y_{1}, y_{2}\right),\left(y_{1}, y_{2}\right)\right\rangle\right\rangle=0 \text {, para todo }\left(y_{1}, y_{2}\right) \in D\left(C_{Y}\right) .
$$

Seja $\left(y_{1}, y_{2}\right) \in D\left(C_{Y}\right)$. Logo, existe um único $z_{2} \in L^{2}(\Omega)$ tal que $\varphi_{z_{2}}=y_{2} \in H^{-1}(\Omega)=$ 
$\left(H_{0}^{1}(\Omega)\right)^{\prime}$. O Teorema de Representação de Riesz implica que existe um $u \in H_{0}^{1}(\Omega)$ tal que $\varphi_{z_{2}}(w)=\langle u, w\rangle_{1}$, para todo $w \in H_{0}^{1}(\Omega)$. Portanto, $f_{u}=\varphi_{z_{2}}$, onde $f_{u}$ é como no Lema 2.14 . Com isso temos

$$
\left\langle\left\langle C_{Y}\left(y_{1}, y_{2}\right),\left(y_{1}, y_{2}\right)\right\rangle\right\rangle=\left\langle z_{2}, y_{1}\right\rangle_{L^{2}(\Omega)}+\left\langle-\Lambda\left(y_{1}\right), y_{2}\right\rangle_{-1} .
$$

Notemos que

$$
\begin{aligned}
\left\langle-\Lambda\left(y_{1}\right), y_{2}\right\rangle_{-1} & =-\left\langle\Lambda^{-1}\left(\Lambda\left(y_{1}\right)\right), \Lambda^{-1}\left(\varphi_{z_{2}}\right)\right\rangle_{1}=-\left\langle y_{1}, u\right\rangle_{1}=-\left\langle u, y_{1}\right\rangle_{1} \\
& =-f_{u}\left(y_{1}\right)=-\varphi_{z_{2}}\left(y_{1}\right)=-\left\langle y_{1}, z_{2}\right\rangle_{L^{2}(\Omega)} .
\end{aligned}
$$

Logo, a igualdade em (2.31) é válida e isso implica que $C_{Y}$ é dissipativo. Usando os mesmos argumentos da prova da Proposição 2.17 $\operatorname{com} \alpha \equiv 0$, mostramos que para qualquer $\lambda \in(0, \infty)$ e $(f, g) \in Y$, existe um $\left(y_{1}, y_{2}\right) \in D\left(C_{Y}\right)$ com $\left(y_{1}, y_{2}\right)-\lambda C_{Y}\left(y_{1}, y_{2}\right)=(f, g)$. E, como na demonstração da Proposição 2.17 temos que $C_{Y}$ é gerador de um $C_{0}$-semigrupo de contração $\left\{S_{Y}(t) \mid t \in[0, \infty)\right\}$ em $Y$.

Segue das definições de $C_{Y}$ e $C_{Z}$ que $v\left(D\left(C_{Z}\right)\right) \subset D\left(C_{Y}\right)$. Afirmamos que

$$
v\left(C_{Z}\left(z_{1}, z_{2}\right)\right)=C_{Y} v\left(z_{1}, z_{2}\right), \text { para todo }\left(z_{1}, z_{2}\right) \in D\left(C_{Z}\right) .
$$

De fato, seja $\left(z_{1}, z_{2}\right) \in D\left(C_{Z}\right)$. Logo, $z_{2} \in H_{0}^{1}(\Omega)$ e $L z_{1}-\beta z_{1}$ (no sentido de distribuições) está em $L^{2}(\Omega)$. Além disso,

$$
v\left(C_{Z}\left(z_{1}, z_{2}\right)\right)=v\left(z_{2},(1 / \varepsilon) L z_{1}-(1 / \varepsilon) \beta z_{1}\right)=\left(z_{2}, \varphi_{(1 / \varepsilon) L z_{1}-(1 / \varepsilon) \beta z_{1}}\right)
$$

Por outro lado,

$$
C_{Y}\left(v\left(z_{1}, z_{2}\right)\right)=C_{Y}\left(z_{1}, \varphi_{z_{2}}\right)=\left(z_{2},-\Lambda\left(z_{1}\right)\right) .
$$

Logo, para concluir a demonstração da igualdade (2.32) basta mostrar que $-\Lambda\left(z_{1}\right)=$ $\varphi_{(1 / \varepsilon) L z_{1}-(1 / \varepsilon) \beta z_{1}}$. Para cada $v \in H_{0}^{1}(\Omega)$, temos

$$
\varphi_{(1 / \varepsilon) L z_{1}-(1 / \varepsilon) \beta z_{1}}(v)=-\frac{1}{\varepsilon}\left\langle A \nabla z_{1}, \nabla v\right\rangle-\frac{1}{\varepsilon}\left\langle\beta z_{1}, v\right\rangle
$$

e

$$
-\Lambda\left(z_{1}\right)(v)=-f_{z_{1}}(v)=-\left\langle z_{1}, v\right\rangle_{1}=-\frac{1}{\varepsilon}\left\langle A \nabla z_{1}, \nabla v\right\rangle-\frac{1}{\varepsilon}\left\langle\beta z_{1}, v\right\rangle .
$$

Agora, uma aplicação Proposição 2.3 finaliza a demonstração do resultado. 
Em vista da Proposição 2.18, dado um $z \in Z$ temos unicamente determinado o elemento $v(z) \in Y$. No restante deste capítulo e no Capítulo 4 iremos usar essa identificação e denotare$\operatorname{mos} v(z)$ por $z$.

Proposição 2.19. Assuma as condições da Hipótese 2.5 e da Hipótese 2.16 Seja $\{T(t) \mid t \geq 0\}$ como na Proposição 2.17 Suponha que exista uma constante $C_{1} \in[0, \infty)$ tal que

$$
|\alpha z|_{H^{-1}(\Omega)} \leq C_{1}|z|_{H^{-1}(\Omega)}, \text { para todo } z \in L^{2}(\Omega)
$$

Então existem constantes $C_{2}, C_{3} \in[0, \infty)$ tais que

$$
|T(t) z|_{Y} \leq C_{2} e^{C_{3} t}|z|_{Y}, t \in[0, \infty), \text { para todo } z \in Z
$$

Demonstração. Defina o operador linear limitado $Q: Z \rightarrow Z$ por $Q\left(z_{1}, z_{2}\right)=\left(0,-\alpha z_{2}\right)$, $\left(z_{1}, z_{2}\right) \in Z$. A Proposição 2.3 e o Teorema 2.6 implicam que para todo $z \in Z$ e para todo $t \in[0, \infty)$,

$$
T(t) z=S_{Z}(t) z+\int_{0}^{t} S_{Z}(t-s) Q T(s) z d s=S_{Y}(t) z+\int_{0}^{t} S_{Y}(t-s) Q T(s) z d s
$$

Além disso, como $\left\{S_{Y}(t) \mid t \in[0, \infty)\right\}$ é $C_{0}$-semigrupo, existem constantes $C_{4}, C_{5} \in[0, \infty)$ tais que

$$
\left|S_{Y}(t) y\right|_{Y} \leq C_{4} e^{C_{5} t}|y|_{Y} \text {, para todo } t \in[0, \infty) \text { e para todo } y \in Y \text {. }
$$

Usando a condição 2.33 obtemos, para $z \in Z$ e $t \in[0, \infty)$,

$$
\begin{aligned}
|T(t) z|_{Y} & \leq\left|S_{Y}(t) z\right|_{Y}+\int_{0}^{t}\left|S_{Y}(t-s) Q T(s) z\right|_{Y} d s \\
& \leq C_{4} e^{C_{5} t}|z|_{Y}+\int_{0}^{t} C_{4} e^{C_{5}(t-s)} C_{1}|T(s) z|_{Y} d s
\end{aligned}
$$

e, portanto, para $z \in Z$ temos

$$
e^{-C_{5} t}|T(t) z|_{Y} \leq C_{4}|z|_{Y}+\int_{0}^{t} C_{4} e^{-C_{5} s} C_{1}|T(t) z|_{Y} d s, \text { para todo } t \in[0, \infty) .
$$

Uma aplicação do Lema de Gronwall (ver Lema 1.61) completa a prova.

O resultado a seguir apresenta uma condição suficiente para que a desigualdade na condição 2.33 seja válida.

Lema 2.20. Sejam $a \in C^{1}(\Omega) \cap W^{1, \infty}(\Omega)$ e $u \in H_{0}^{1}(\Omega)$. Então $a u \in H_{0}^{1}(\Omega)$ e $\partial_{i}(a u)=\left(\partial_{i} a\right) u+$ 
$a \partial_{i} u, i \in\{1, \ldots, N\}$. Além disso,

$$
|a u|_{H_{0}^{1}(\Omega)} \leq(2 N+1)^{1 / 2}|a|_{W^{1, \infty}(\Omega)}|u|_{H_{0}^{1}(\Omega)}
$$

Mais ainda,

$$
|a z|_{H^{-1}(\Omega)} \leq(2 N+1)^{1 / 2}|a|_{W^{1, \infty}(\Omega)}|z|_{H^{-1}(\Omega)} \text {, para todo } z \in L^{2}(\Omega),
$$

Se U é um subconjunto aberto de $\Omega$ e a $\left.\right|_{U} \in C_{0}^{1}(U)$, então $\left.(a u)\right|_{U} \in H_{0}^{1}(\Omega)$.

Demonstração. Para cada $i \in\{1, \ldots, N\}$, defina $u_{(i)}=\left(\partial_{i} a\right) u+a \partial_{i} u$. A definição do espaço $H_{0}^{1}(\Omega)$ implica que existe uma sequência $\left(v_{n}\right)_{n}$ em $C_{0}^{1}(\Omega)$ convergindo para $u$ em $H^{1}(\Omega)$. Segue que $a v_{n} \in C_{0}^{1}(\Omega)$ e $\partial_{i}\left(a v_{n}\right)=\left(\partial_{i} a\right) v_{n}+a \partial_{i} v_{n}$, para todo $n \in \mathbb{N}$ e para todo $i \in\{1, \ldots, N\}$.

Seja $i \in\{1, \ldots, N\}$. Como $a \in C^{1}(\Omega) \cap W^{1, \infty}(\Omega)$ temos que

$$
\begin{aligned}
{\left[\int_{\Omega}\left|a(x) v_{n}(x)-a(x) u(x)\right|^{2} d x\right]^{1 / 2} } & \leq|a|_{W^{1, \infty}(\Omega)}\left[\int_{\Omega}\left|v_{n}(x)-u(x)\right|^{2} d x\right]^{1 / 2} \\
& \leq|a|_{W^{1, \infty}(\Omega)}\left|v_{n}-u\right|_{H^{1}(\Omega)} .
\end{aligned}
$$

Como $\left|v_{n}-u\right|_{H^{1}(\Omega)} \rightarrow 0$ quando $n \rightarrow \infty$, segue que

$$
\left[\int_{\Omega}\left|a(x) v_{n}(x)-a(x) u(x)\right|^{2} d x\right]^{1 / 2} \rightarrow 0 \text { quando } n \rightarrow \infty .
$$

Também

$$
\begin{aligned}
{\left[\int_{\Omega}\left|\partial_{i}\left(a v_{n}\right)(x)-u_{(i)}(x)\right|^{2}\right]^{1 / 2} } & =\left[\int_{\Omega}\left|\left(\partial_{i} a(x)\right)\left(v_{n}(x)-u(x)\right)+a(x)\left(\partial_{i}\left(v_{n}(x)-u(x)\right)\right)\right|^{2} d x\right]^{1 / 2} \\
& \leq|a|_{W^{1, \infty}(\Omega)}\left[\int_{\Omega}\left|\left[v_{n}(x)-u(x)\right]+\left[\partial_{i}\left(v_{n}(x)-u(x)\right)\right]\right|^{2} d x\right]^{1 / 2} \\
& =|a|_{W^{1, \infty}(\Omega)}\left|v_{n}-u\right|_{H^{1}(\Omega)}
\end{aligned}
$$

o que implica que

$$
\left[\int_{\Omega}\left|\partial_{i}\left(a v_{n}\right)(x)-u_{(i)}(x)\right|^{2}\right]^{1 / 2} \rightarrow 0 \text { quando } n \rightarrow \infty .
$$

Fórmulas 2.36) e 2.37) implicam que $\left(a v_{n}\right)_{n}$ converge para au em $L^{2}(\Omega)$ e que $\left(\partial_{i}\left(a v_{n}\right)\right)_{n}$ converge para $u_{(i)}$ em $L^{2}(\Omega)$. Além disso, se $\varphi \in C_{0}^{1}(\Omega)$, usando a Desigualdade de Hölder, 
obtemos

$$
\begin{aligned}
& \int_{\Omega}\left|\varphi(x) \partial_{i}\left(a v_{n}\right)(x)-\varphi(x) u_{(i)}(x)\right| d x=\int_{\Omega}\left|\varphi(x)\left(\left(a v_{n}\right)(x)-u_{i}(x)\right)\right| \\
& \quad \leq\left[\int_{\Omega}|\varphi(x)|^{2} d x\right]^{1 / 2}\left[\int_{\Omega}\left|\partial_{i}\left(a v_{n}\right)(x)-u_{i}(x)\right|^{2} d x\right]^{1 / 2}=|\varphi|_{L^{2}(\Omega)}\left|\partial_{i}\left(a v_{n}\right)-u_{i}\right|_{L^{2}(\Omega)}
\end{aligned}
$$

e

$$
\begin{aligned}
& \int_{\Omega}\left|\left(\partial_{i} \varphi(x)\right)\left(a v_{n}\right)(x)-\left(\partial_{i} \varphi(x)\right)(a u)(x)\right| d x=\int_{\Omega}\left|\left(\partial_{i} \varphi(x)\right)\left(\left(a v_{n}\right)(x)-(a u)(x)\right)\right| d x \\
& \leq\left[\int_{\Omega}\left|\partial_{i} \varphi(x)\right|^{2} d x\right]^{1 / 2}\left[\int_{\Omega}\left|\left(a v_{n}\right)(x)-(a u)(x)\right|^{2} d x\right]^{1 / 2}=\left|\partial_{i} \varphi\right|_{L^{2}(\Omega)}\left|a v_{n}-a u\right|_{L^{2}(\Omega)} .
\end{aligned}
$$

Logo, $\left(\varphi \partial_{i}\left(a v_{n}\right)\right)_{n}$ converge para $\varphi u_{(i)}$ em $L^{1}(\Omega)$ e $\left(\partial_{i} \varphi\left(a v_{n}\right)\right)_{n}$ converge para $\partial_{i} \varphi(a u)$ em $L^{1}(\Omega)$. Como

$$
\left\langle\varphi, \partial_{i}\left(a v_{n}\right)\right\rangle_{L^{2}(\Omega)}=-\left\langle\partial_{i} \varphi, a v_{n}\right\rangle_{L^{2}(\Omega)} \text { para todo } n \in \mathbb{N},
$$

temos que $a u \in H^{1}(\Omega), \partial_{i}(a u)=u_{(i)} \mathrm{e}$

$$
\left|a v_{n}-a u\right|_{H^{1}(\Omega)} \rightarrow 0 \text { quando } n \rightarrow \infty
$$

Portanto, $a u \in H_{0}^{1}(\Omega)$, o que prova a primeira parte do lema. Para mostrar a desigualdade 2.34 notemos que

$$
\begin{aligned}
|a u|_{H_{0}^{1}(\Omega)}^{2} & =|a u|_{L^{2}(\Omega)}^{2}+\sum_{i=1}^{N}\left|\partial_{i}(a u)\right|_{L^{2}(\Omega)}^{2}=|a u|_{L^{2}(\Omega)}^{2}+\sum_{i=1}^{N}\left|\left(\partial_{i} a\right) u+a \partial_{i} u\right|_{L^{2}(\Omega)}^{2} \\
& \leq|a|_{W^{1, \infty}(\Omega)}^{2}|u|_{L^{2}(\Omega)}^{2}+\sum_{i=1}^{N}|a|_{W^{1, \infty}(\Omega)}^{2}\left(|u|_{L^{2}(\Omega)}+\left|\partial_{i} u\right|\right)_{L^{2}(\Omega)}^{2} \\
& =|a|_{W^{1, \infty}(\Omega)}^{2}\left(|u|_{L^{2}(\Omega)}^{2}+\sum_{i=1}^{N}\left(|u|_{L^{2}(\Omega)}+\left|\partial_{i} u\right|_{L^{2}(\Omega)}\right)^{2}\right) \\
& \leq|a|_{W^{1, \infty}(\Omega)}^{2}\left(|u|_{L^{2}(\Omega)}^{2}+\sum_{i=1}^{N}\left(2|u|_{L^{2}(\Omega)}^{2}+2\left|\partial_{i} u\right|_{L^{2}(\Omega)}^{2}\right)\right) \\
& =|a|_{W^{1, \infty}(\Omega)}^{2}\left((2 N-1)|u|_{L^{2}(\Omega)}^{2}+2\left(|u|_{L^{2}(\Omega)}^{2}+\sum_{i=1}^{N}\left|\partial_{i} u\right|_{L^{2}(\Omega)}^{2}\right)\right. \\
& =|a|_{W^{1, \infty}(\Omega)}^{2}\left((2 N-1)|u|_{L^{2}(\Omega)}^{2}+2|u|_{H_{0}^{1}(\Omega)}^{2}\right) \\
& \leq|a|_{W^{1, \infty}(\Omega)}^{2}(2 N+1)|u|_{H_{0}^{1}(\Omega)}^{2} .
\end{aligned}
$$

Para concluir a demonstração, mostremos a desigualdade (2.35). Seja $z \in L^{2}(\Omega)$. Segue que 
$a z \in L^{2}(\Omega)$. Além disso para todo $v \in H_{0}^{1}(\Omega)$ com $|v|_{H^{1}} \leq 1$, temos que $a v \in H_{0}^{1}(\Omega) \mathrm{e}$

$$
\left|\langle a z, v\rangle_{L^{2}(\Omega)}\right|=\left|\langle z, a v\rangle_{L^{2}(\Omega)}\right| \leq|z|_{H^{-1}(\Omega)}|a v|_{H^{1}(\Omega)} \leq(2 N+1)^{1 / 2}|a|_{W^{1, \infty}(\Omega)}|z|_{H^{-1}(\Omega)} .
$$

Finalmente, se $\left.a\right|_{U} \in C_{0}^{1}(U)$, então $\left.\left(a v_{n}\right)\right|_{U} \in C_{0}^{1}(U)$ para todo $n \in \mathbb{N}$ e, portanto, segue de (2.38), que a sequência $\left(\left.\left(a v_{n}\right)\right|_{U}\right)_{n}$ converge para $\left.(a u)\right|_{U}$ em $H^{1}(U)$, de onde segue que $\left.(a u)\right|_{U} \in H_{0}^{1}(\Omega)$. 


\section{Capítulo}

\section{3}

\section{A definição do semifluxo gerado pelas soluções da equação da onda amortecida}

Neste capítulo apresentaremos condições (ver Hipótese 3.9 e Proposição 3.10) para que as soluções da equação da onda amortecida,

$$
\begin{array}{rlrl}
\varepsilon u_{t t}+\alpha(x) u_{t}+\beta(x) u-\sum_{i, j} \partial_{i}\left(a_{i j}(x) \partial_{j} u\right) & =f(x, u), & & x \in \Omega, t \in[0, \infty), \\
u(x, t) & =0, & x \in \partial \Omega, t \in[0, \infty)
\end{array}
$$

gerem um semifluxo local.

Iniciamos o capítulo com um resultado auxiliar sobre a existência de um semifluxo local para uma equação integral.

\subsection{Um resultado preliminar}

Nssa seção demonstramos um resultado sobre equações semilineares definidas por um $C_{0^{-}}$ semigrupo.

Proposição 3.1. Seja $Z$ um espaço de Banach e $\{T(t) \mid t \in[0, \infty)\}$ um $C_{0}$-semigrupo de operadores lineares em $Z$, com gerador infinitesimal $B: D(B) \subset Z \rightarrow Z$. Suponha $\Phi: Z \rightarrow Z$ seja uma aplicação Lipschitziana em conjuntos limitados de $Z$. Então, para cada $\zeta \in Z$, existe um $\omega_{\zeta}=\omega_{B, \Phi, \zeta} \in(0, \infty]$ e uma aplicação contínua $z=z_{\zeta}:\left[0, \omega_{\zeta}\right) \rightarrow Z$ unicamente determinada tal que

$$
z(t)=T(t) \zeta+\int_{0}^{t} T(t-s) \Phi(z(s)) d s, t \in\left[0, \omega_{\zeta}\right)
$$


Escrevendo $\zeta \pi t:=z_{\zeta}(t), t \in\left[0, \omega_{\zeta}\right)$, obtemos um semifluxo local $\pi=\pi_{B, \Phi}$ em $Z$ que não explode em subconjuntos limitados de Z.

Demonstração. Uma aplicação do Teorema 1.63 implica que existe uma função $\omega: Z \rightarrow(0, \infty]$ tal que para cada $x \in Z$ existe uma única função $z=z_{x} \in C([0, \omega(x)), Z)$ com a propriedade: para todo $T$ com $0<T<\omega(x)$, temos

$$
z(t)=T(t) x+\int_{0}^{t} T(t-s) \Phi(z(s)) d s, t \in[0, T]
$$

Mostremos agora que $x \pi t:=z_{x}(t)$, para $t \in[0, \omega(x))$ e $x \in Z$, determina um semifluxo local. Para tanto, primeiro note que $x \pi 0=z_{x}(0)=x$. Além disso, note que a Proposição 1.64 implica que $\pi$ assim definida é contínua. No que segue para cada $x \in Z, \omega_{x}$ denota $\omega(x)$. Resta verificar a condição (iii) da Definição 1.39 . Sejam $x \in Z, t \in\left[0, \omega_{x}\right)$ e $s \in\left[0, \omega_{x \pi t}\right)$. Devemos mostrar que $t+s \in\left[0, \omega_{x}\right)$ e $x \pi(t+s)=(x \pi t) \pi s$.

Afirmamos que $t+s \in\left[0, \omega_{x}\right)$. Se $\omega_{x}=\infty$, isto está provado. Caso contrário, suponha, por absurdo, que $t<\omega_{x} \leq t+s<\omega_{x \pi t}$. Logo, existe um $\tau \geq 0$ tal que $\omega_{x} \leq \tau \leq t+s$. Disso segue que $0<\omega_{x}-t \leq \tau-t \leq s<\omega_{x \pi t}-t<\omega_{x \pi t}$. Defina $\varphi \in C([0, t+s], Z)$ por

$$
\varphi(\tau)= \begin{cases}u(\tau) & \text { se } \tau \in[0, t] \\ v(\tau-t) & \text { se } \tau \in[t, t+s]\end{cases}
$$

onde $u \in C\left(\left[0, \omega_{x}\right), Z\right)$ é dado por

$$
u(t)=T(t) x+\int_{0}^{t} T(t-s) \Phi(u(s)) d s, t \in\left[0, \omega_{x}\right)
$$

e $v \in C\left(\left[0, \omega_{x \pi t}\right), Z\right)$ é a solução dada pela Proposição 1.62 da equação integral

$$
v(s)=T(s) u(t)+\int_{0}^{s} T(s-\sigma) \Phi(v(\sigma)) d \sigma, s \in\left[0, \omega_{x \pi t}\right) .
$$

Vemos que, de modo análogo ao feito na demonstração da Proposição 1.63 , temos que

$$
\varphi(t)=T(t) x+\int_{0}^{t} T(t-s) \phi(\varphi(s)) d s, \quad t \in[0, \omega]
$$

com $\omega=t+s$, o que contradiz a definição de $\omega_{x}$, pois $t+s>\omega_{x}$. Logo, $t+s<\omega_{x}$. Finalmente, defina $\psi \in C([0, t+s], Z)$ por

$$
\psi(\tau)= \begin{cases}x \pi \tau & \text { se } \tau \in[0, t] \\ (x \pi t) \pi(\tau-t) & \text { se } \tau \in[t, t+s]\end{cases}
$$


A unicidade de soluções da equação integral (1.4) com $\psi(0)=x$ e $\omega=t+s$ implica que $x \pi(t+s)=(x \pi t) \pi s$. Portanto, $\pi$ é um semifluxo local. A propriedade de não explosão em subconjuntos limitados de $Z$ segue do Teorema 1.63 parte $(i i)$.

\subsection{O semifluxo gerado pelas soluções da equação da onda amortecida}

Denote por $\mathscr{M}$ o conjunto das funções $v: \Omega \rightarrow \mathbb{R}$ Lebesgue-mensuráveis.

Definição 3.2. Uma função $f: \Omega \times \mathbb{R} \rightarrow \mathbb{R},(x, u) \mapsto f(x, u)$ satisfaz a condição $C^{0}$ (respectivamente $C^{1}$ ) de Carathéodory se:

(i) para todo $u \in \mathbb{R}$, a aplicação $x \mapsto f(x, u)$ é Lebesgue-mensurável;

(ii) para quase todo $x \in \Omega$, a aplicação $u \mapsto f(x, u)$ é contínua (respectivamente continuamente diferenciável).

Seja $f: \Omega \times \mathbb{R} \rightarrow \mathbb{R},(x, u) \mapsto f(x, u)$, uma função que satisfaz a condição $C^{0}$ de Carathéodory. Defina a função $F: \Omega \times \mathbb{R} \rightarrow \mathbb{R}$ por

$$
F(x, u)=\int_{0}^{u} f(x, s) d s
$$

onde $s \mapsto f(x, s)$ é contínua e $F(x, u)=0$ caso contrário. A função $F$ é chamada a primitiva canônica de $f$.

Definição 3.3. Sejam $\bar{C}, \bar{\rho} \in[0, \infty), a: \Omega \rightarrow \mathbb{R}$ uma função mensurável $e M \subset \Omega$ um conjunto de medida nula. Dizemos que uma função $g:(\Omega \backslash M) \times \mathbb{R} \rightarrow \mathbb{R},(x, u) \mapsto g(x, u)$, satisfaz a uma condição $(\bar{C}, \bar{\rho}, a)$ de crescimento se

$$
|g(x, u)| \leq \bar{C}\left(|a(x)|+|u|^{\bar{\rho}}\right), \text { para todo } x \in \Omega \backslash M \text { e para todo } u \in \mathbb{R} \text {. }
$$

O número $\bar{\rho}$ na Definição 3.3 é chamado subcrítico se $N \leq 2$ ou $\left(N \geq 3\right.$ e $\left.\bar{\rho}<\left(2^{*} / 2\right)-1\right)$. É chamado crítico se $N \geq 3$ e $\bar{\rho}=\left(2^{*} / 2\right)-1$.

Observação 3.4. Dada uma função Lebesgue-mensurável $v: \Omega \rightarrow \mathbb{R}$ as funções $x \in \Omega \mapsto$ $f(x, v(x)) \in \mathbb{R}$ e $x \in \Omega \mapsto F(x, v(x)) \in \mathbb{R}$ são Lebesgue-mensuráveis. 
Definição 3.5. Seja $f: \Omega \times \mathbb{R} \rightarrow \mathbb{R}$ uma função que satisfaz a condição $C_{0}$ de Carathéodory. $A$ aplicação $\widehat{f}: \mathscr{M} \rightarrow \mathscr{M}$ definida por $\widehat{f}(u)(x)=f(x, u(x)), u \in \mathscr{M}, x \in \Omega$, é chamada operador de Nemytskii associado a $f$.

Proposição 3.6. Seja $f: \Omega \times \mathbb{R} \rightarrow \mathbb{R}$ uma função satisfazendo a condição $C^{1}$ de Carathéodory. Suponha que existam constantes $\bar{C}, \bar{\rho} \in[0, \infty)$, uma função mensurável a: $\Omega \rightarrow \mathbb{R}$ e um conjunto de medida nula $M \subset \Omega$ tais que a função $\partial_{u} f$ satisfaz a condição $(\bar{C}, \bar{\rho}$, a) de crescimento. Seja $F$ a primitiva canônica de $f$. Então, para quase todo $x \in \Omega$ e para todo $u, h \in \mathbb{R}$, temos

$$
\begin{gathered}
|f(x, u)-f(x, 0)| \leq \bar{C}\left(|a(x)||u|+|u|^{\bar{\rho}+1}\right), \\
|f(x, u+h)-f(x, u)| \leq \bar{C}|a(x)||h|+\bar{C} \max \left(1,2^{\bar{\rho}-1}\right)\left(|u|^{\bar{\rho}}+|h|^{\bar{\rho}}\right)|h|, \\
|F(x, u)| \leq \bar{C}\left(|a(x)||u|^{2} / 2+|u|^{\bar{\rho}+2} /(\bar{\rho}+2)\right)+|u||f(x, 0)|, \\
|F(x, u+h)-F(x, u)| \\
\leq\left(|f(x, 0)|+\bar{C}|a(x)|(|u|+|h|)+\bar{C} \max \left(1,2^{\bar{\rho}}\right)\left(|u|^{\bar{\rho}+1}+|h|^{\bar{\rho}+1}\right)\right)|h|
\end{gathered}
$$

$e$

$$
|F(x, u+h)-F(x, u)-f(x, u) h| \leq\left(\bar{C}|a(x)|+\bar{C} \max \left(1,2^{\bar{\rho}-1}\right)\left(|u|^{\bar{\rho}}+|h|^{\bar{\rho}}\right)\right)|h|^{2}
$$

Demonstração. Para quase todo $x \in \Omega$ e para todo $u, h \in \mathbb{R}$ temos

$$
f(x, u+h)-f(x, u)=\int_{0}^{1} \partial_{u} f(x, u+\theta h) h d \theta .
$$

Em particular se $h=-u$ temos

$$
f(x, 0)-f(x, u)=-\int_{0}^{1} \partial_{u} f(x, \theta u) u d \theta
$$

e, assim,

$$
|f(x, u)-f(x, 0)| \leq \int_{0}^{1}\left|\partial_{u} f(x, \theta u)\right||u| d \theta
$$

Como $\partial_{u} f$ satisfaz a condição $(\bar{C}, \bar{\rho}, a)$ de crescimento, temos que

$$
\left|\partial_{u} f(x, u)\right| \leq \bar{C}\left(|a(x)|+|u|^{\bar{\rho}}\right) \text {, para todo } x \in \Omega \backslash M \text { e para todo } u \in \mathbb{R} \text {. }
$$


Logo

$$
|f(x, u)-f(x, 0)| \leq \int_{0}^{1} \bar{C}\left(|a(x)|+|u|^{\bar{\rho}}\right)|u| d \theta \leq \bar{C}|a(x)||u|+\bar{C}|u|^{\bar{\rho}+1}
$$

e a desigualdade (3.1) está demonstrada. Segue da igualdade (3.6) que

$$
|f(x, u+h)-f(x, u)| \leq \int_{0}^{1}\left|\partial_{u} f(x, u+\theta h)\right||h| d \theta
$$

para quase todo $x \in \Omega$ e para todo $u, h \in \mathbb{R}$. A desigualdade (3.7) implica que

$$
\begin{aligned}
|f(x, u+h)-f(x, u)| & \leq \int_{0}^{1} \bar{C}\left(|a(x)|+|u+\theta h|^{\bar{\rho}}\right)|h| d \theta \\
& \leq \bar{C}|a(x)||h|+\bar{C} \max \left(1,2^{\bar{\rho}-1}\right)\left(|u|^{\bar{\rho}}+|h|^{\bar{\rho}}\right)|h|
\end{aligned}
$$

o que mostra a desigualdade (3.2). Para mostrar a desigualdade (3.3) notemos que para quase todo $x \in \Omega$ e para todo $u, h \in \mathbb{R}$ temos

$$
\begin{aligned}
|F(x, u)| & \leq \int_{0}^{u}|f(x, s)| d s=\int_{0}^{u}\left|\int_{0}^{1} \partial f(x, \theta s) s d \theta+f(x, 0)\right| d s \\
& \leq \int_{0}^{u} \int_{0}^{1}|\partial f(x, \theta s)||s| d \theta+\int_{0}^{u}|f(x, 0)| d s \leq \int_{0}^{u} \bar{C}\left(|a(x)|+|s|^{\bar{\rho}}\right)|s| d s+|f(x, 0)||u| \\
& =\bar{C}|a(x)||u|^{2} / 2+\bar{C}|u|^{\overline{\rho+2}} /(\bar{\rho}+2)+|f(x, 0) \| u| .
\end{aligned}
$$

Aqui novamente utilizamos a igualdade (3.6) $\operatorname{com} h=-u$ e a desigualdade (3.7). Além disso, essas condições também implicam que

$$
\begin{aligned}
\mid F(x, u+h) & -F(x, u)\left|\leq \int_{u}^{u+h}\right| f(x, s)\left|d s=\int_{u}^{u+h}\right| \int_{0}^{1} \partial_{u} f(x, \theta s) s d \theta+f(x, 0) \mid d s \\
& \leq \int_{u}^{u+h} \int_{0}^{1}\left|\partial_{u} f(x, \theta s)\right||s| d \theta+\int_{u}^{u+h}|f(x, 0)| d s \\
& \leq \int_{u}^{u+h} \bar{C}\left(|a(x)|+|s|^{\bar{\rho}}\right)|s| d s+|f(x, 0)||h| \\
& \leq|f(x, 0)||h|+\bar{C}|a(x)|\left(\frac{|u+h|^{2}-|u|^{2}}{2}\right)|h|+\int_{0}^{1} \bar{C}|u+\delta h|^{\bar{\rho}+1}|h| d \delta \\
& \leq|f(x, 0)||h|+\bar{C}|a(x)|(|u|+|h|)|h|+\bar{C} \max \left(1,2^{\bar{\rho}}\right)\left(|u|^{\bar{\rho}+1}+|h|^{\bar{\rho}+1}\right)|h|
\end{aligned}
$$

e desigualdade (3.4) está verificada. Finalmente para mostrar a desigualdade (3.5) notemos que 
para quase todo $x \in \Omega$ e para todo $u, h \in \mathbb{R}$ temos

$$
\begin{aligned}
F(x, u+h)-F(x, u)-f(x, u) h & =\int_{0}^{1}[f(x, u+\theta h)-f(x, u)] h d \theta \\
& =\int_{0}^{1}\left[\int_{0}^{1} \partial_{u} f(x, u+r \theta h) \theta h d r\right] h d \theta
\end{aligned}
$$

Uma aplicação da desigualdade (3.7) implica que

$$
\begin{aligned}
\mid F(x, u+h) & -F(x, u)-f(x, u) h\left|\leq \int_{0}^{1} \int_{0}^{1}\right| \partial_{u} f(x, u+r \theta h)|| \theta h|d r| h \mid d \theta \\
& \leq \int_{0}^{1} \int_{0}^{1} \bar{C}\left(|a(x)|+|u+r \theta h|^{\bar{\rho}}\right)|\theta||h| d r|h| d \theta \\
& \leq \int_{0}^{1} \bar{C}|a(x)||\theta||h|^{2} d \theta+\int_{0}^{1} \bar{C} \max \left(1,2^{\bar{\rho}-1}\right)\left(|u|^{\bar{\rho}}+|\theta h|^{\bar{\rho}}\right)|\theta||h|^{2} d \theta \\
& \leq \bar{C}|a(x)||h|^{2}+\bar{C} \max \left(1,2^{\bar{\rho}-1}\right)\left(|u|^{\bar{\rho}}+|h|^{\bar{\rho}}\right)|h|^{2} .
\end{aligned}
$$

Assim, a proposição está demonstrada.

A partir da Proposição 3.6 obteremos estimativas para os operadores de Nemytskii associados às funções $f$ e $F$.

Para demonstrar a próxima proposição, usaremos o seguinte lema clássico.

Lema 3.7 (Extensão da Desigualdade de Hölder). Sejam $f_{i}: \Omega \rightarrow \mathbb{R}, i \in\{1, \ldots, k\}$ funções tais que para cada $i \in\{1, \ldots, k\}$ existe um $1 \leq p_{i}<\infty$ tal que $f_{i} \in L^{p_{i}}(\Omega)$. Se $\sum_{i=1}^{k} \frac{1}{p_{i}} \leq 1$, então $f=f_{1} \cdot f_{2} \cdot \ldots \cdot f_{k} \in L^{p}(\Omega) e$

$$
|f|_{L^{p}(\Omega)} \leq\left|f_{1}\right|_{L^{p_{1}(\Omega)}}\left|f_{1}\right|_{L^{p_{2}(\Omega)}} \ldots\left|f_{1}\right|_{L^{p_{k}(\Omega)}},
$$

onde $\frac{1}{p}=\sum_{i=1}^{k} \frac{1}{p_{i}}$

Com isso, podemos provar o seguinte resultado:

Proposição 3.8. Assuma as hipóteses da Proposição 3.6 Seja $\widehat{f}$ o operador de Nemytskii associado a f e $\widehat{F}$ o operador de Nemytskii associado a $F$. Para todas as funções mensuráveis $u, h \in \mathscr{M}$ temos

$$
|\widehat{f}(u)|_{L^{2}(\Omega)} \leq|\widehat{f}(0)|_{L^{2}(\Omega)}+\bar{C}\left(|a u|_{L^{2}(\Omega)}+|u|_{L^{2(\bar{\rho}+1)}}^{\bar{\rho}+1}\right)
$$




$$
\begin{aligned}
& \mid \widehat{f}(u+h)-\left.\widehat{f}(u)\right|_{L^{2}(\Omega)} \\
& \leq \bar{C}|a h|_{L^{2}(\Omega)}+\bar{C} \max \left(1,2^{\bar{\rho}-1}\right)\left(|u|_{L^{2(\bar{\rho}+1)}(\Omega)}^{\bar{\rho}}+|h|_{L^{2(\bar{\rho}+1)}(\Omega)}^{\bar{\rho}}\right)|h|_{L^{2(\bar{\rho}+1)}(\Omega)} \\
&|\widehat{F}(u)|_{L^{1}(\Omega)} \leq \bar{C}\left(\left.\left.|a| u\right|^{2}\right|_{L^{1}(\Omega)} / 2+|u|_{L^{\bar{\rho}+2}(\Omega)}^{\bar{\rho}+2} /(\bar{\rho}+2)\right)+|u|_{L^{2}(\Omega)}|\widehat{f}(0)|_{L^{2}(\Omega)} \\
&|\widehat{F}(u+h)-\widehat{F}(u)|_{L^{1}(\Omega)} \leq\left(|\widehat{f}(0)|_{L^{2}(\Omega)}+\bar{C}\left(|a u|_{L^{2}(\Omega)}+|a h|_{L^{2}(\Omega)}\right)\right)|h|_{L^{2}(\Omega)} \\
&+\left(\bar{C} \max \left(1,2^{\bar{\rho}}\right)\left(|u|_{L^{2(\bar{\rho}+1)(\Omega)}}^{\bar{\rho}+1}+|h|_{L^{2(\bar{\rho}+1)}(\Omega)}^{\bar{\rho}+1}\right)\right)|h|_{L^{2}(\Omega)}
\end{aligned}
$$

e

$$
\begin{aligned}
\mid \widehat{F}(u+h) & -\widehat{F}(u)-\left.\widehat{f}(u) h\right|_{L^{1}(\Omega)} \leq \bar{C}|a h|_{L^{2}(\Omega)}|h|_{L^{2}(\Omega)} \\
& +\bar{C} \max \left(1,2^{\bar{\rho}-1}\right)\left(|u|_{L^{2(\bar{\rho}+1)}(\Omega)}^{\bar{\rho}}+|h|_{L^{2(\bar{\rho}+1)}(\Omega)}^{\bar{\rho}}\right)|h|_{L^{2(\bar{\rho}+1)}(\Omega)}|h|_{L^{2}(\Omega)}
\end{aligned}
$$

Finalmente, se $\bar{\rho}$ é crítico, então para cada $r \in[N, \infty)$ existe uma constante $C(r) \in[0, \infty)$ tal que para toda função a da forma $a=a_{1}+a_{2}$, com $a_{1} \in L^{r}(\Omega)$ e $a_{2} \in L^{\infty}(\Omega)$, temos

$$
\begin{aligned}
|\widehat{f}(u+h)-\widehat{f}(u)|_{H^{-1}(\Omega)} & \leq C(r)\left(\left|a_{1}\right|_{L^{r}(\Omega)}+\left|a_{2}\right|_{L^{\infty}(\Omega)}\right)|h|_{L^{2}(\Omega)} \\
& +C(r)\left(|u|_{L^{2^{*}}(\Omega)}^{\bar{\rho}}+|h|_{L^{2^{*}}(\Omega)}^{\bar{\rho}}\right)|h|_{L^{2}(\Omega)}
\end{aligned}
$$

para todo $u, h \in H_{0}^{1}(\Omega)$.

Demonstração. Recordemos que dada uma função mensurável $u: \Omega \rightarrow \mathbb{R}$, os operadores de Nemytskii $\widehat{f}$ e $\widehat{F}$ são dados por $\widehat{f}(u)(x)=f(x, u(x))$ e $\widehat{F}(u)(x)=F(x, u(x)), x \in \Omega$. Seja $u \in \mathscr{M}$.

Notemos que

$$
|\widehat{f}(u)|_{L^{2}(\Omega)}=\left[\int_{\Omega}|f(x, u(x))|^{2} d x\right]^{1 / 2}=\left[\int_{\Omega}\left|\int_{0}^{1} \partial_{u} f(x, \theta u(x)) u(x) d \theta+f(x, 0)\right|^{2} d x\right]^{1 / 2}
$$

Portanto

$$
|\widehat{f}(u)|_{L^{2}(\Omega)} \leq\left[\int_{\Omega}\left|\int_{0}^{1} \partial_{u} f(x, \theta u(x)) u(x) d \theta\right|^{2} d x\right]^{1 / 2}+\left[\int_{\Omega}|f(x, 0)|^{2} d x\right]^{1 / 2}
$$

Como $\partial_{u} f$ satisfaz a condição $(\bar{C}, \bar{\rho}, a)$ de crescimento temos que

$$
\left|\partial_{u} f(x, u)\right| \leq \bar{C}\left(|a(x)|+|u|^{\bar{\rho}}\right), \text { para todo } x \in \Omega \backslash M \text { e para todo } u \in \mathbb{R}
$$


e, assim,

$$
\begin{aligned}
|\widehat{f}(u)|_{L^{2}(\Omega)} & \leq|\widehat{f}(0)|_{L^{2}(\Omega)}+\left[\int_{\Omega}\left|\int_{0}^{1}\right| \partial_{u} f(x, \theta u(x))|| u(x)|d \theta|^{2} d x\right]^{1 / 2} \\
& \leq|\widehat{f}(0)|_{L^{2}(\Omega)}+\bar{C}\left[\left.\left.\int_{\Omega}|| a(x) u(x)|+| u(x)\right|^{\bar{\rho}+1}\right|^{2} d x\right]^{1 / 2} .
\end{aligned}
$$

Portanto,

$$
\begin{aligned}
|\widehat{f}(u)|_{L^{2}(\Omega)} & \leq|\widehat{f}(0)|_{L^{2}(\Omega)}+\bar{C}\left[\left(\int_{\Omega}|a(x) u(x)|^{2} d x\right)^{1 / 2}+\left(\int_{\Omega}|u(x)|^{2(\bar{\rho}+1)} d x\right)^{1 / 2}\right] \\
& =|\widehat{f}(0)|_{L^{2}(\Omega)}+\bar{C}\left(|a u|_{L^{2}(\Omega)}+|u|_{L^{2(\bar{\rho}+1)}(\Omega)}^{\bar{\rho}+1}\right) .
\end{aligned}
$$

A desigualdade (3.8) está demonstrada. Para mostrar a desigualdade (3.9) notemos que para cada $h \in \mathscr{M}$ segue, da desigualdade (3.2), que

$$
\begin{aligned}
\mid \widehat{f}(u+h) & -\left.\widehat{f}(u)\right|_{L^{2}(\Omega)}=\left[\int_{\Omega}|f(x, u(x)+h(x))-f(x, u(x))|^{2} d x\right]^{1 / 2} \\
& \leq\left[\int_{\Omega}|\bar{C}| a(x)|| h(x)\left|+\bar{C} \max \left(1,2^{\bar{\rho}-1}\right)\left(|u(x)|^{\bar{\rho}}+|h(x)|^{\bar{\rho}}\right)\right| h(x)||^{2} d x\right]^{1 / 2} \\
& \leq \bar{C}\left[\int_{\Omega}|a(x) h(x)|^{2} d x\right]^{1 / 2}+\bar{C} \max \left(1,2^{\bar{\rho}-1}\right)\left[\int_{\Omega}\left(|u(x)|^{\bar{\rho}}+|h(x)|^{\bar{\rho}}\right)^{2}|h(x)|^{2} d x\right]^{1 / 2} \\
& \leq\left(\bar{C}|a h|_{L^{2}(\Omega)}+\bar{C} \max \left(1,2^{\bar{\rho}-1}\right)\left(|u|_{L^{2(\bar{\rho}+1)(\Omega)}}^{\overline{\bar{\rho}}}+|h|_{L^{2(\bar{\rho}+1)}(\Omega)}^{\bar{\rho}}\right)|h|_{L^{2}(\Omega)}\right.
\end{aligned}
$$

Além disso, utilizando a desigualdade 3.3 obtemos

$$
\begin{aligned}
\int_{\Omega}|\widehat{F}(u)| d x & =\int_{\Omega}|F(x, u(x))| d x \\
& \leq \int_{\Omega}\left(\bar{C}\left(|a(x)||u(x)|^{2} / 2+|u(x)|^{\bar{\rho}+2} /(\bar{\rho}+2)\right)+|u(x)||f(x, 0)|\right) d x \\
& =\int_{\Omega} \bar{C}|a(x)||u(x)|^{2} / 2 d x+\int_{\Omega} \bar{C}|u(x)|^{\bar{\rho}+2} /(\bar{\rho}+2)+\int_{\Omega}|\widehat{f}(0)||u(x)| d x \\
& \leq \bar{C}\left(\left.\left.|a| u\right|^{2}\right|_{L^{1}(\Omega)} / 2+|u|_{L^{\bar{\rho}+2}(\Omega)}^{\bar{\rho}+2} /(\bar{\rho}+2)\right)+|u|_{L^{2}(\Omega)}|\widehat{f}(0)|_{L^{2}(\Omega)} .
\end{aligned}
$$

e a prova da desigualdade (3.10) está concluída. Seja $h \in \mathscr{M}$. A desigualdade (3.4) implica que para quase todo $x \in \Omega$

$$
\begin{aligned}
|F(x, u(x)+h(x))-F(x, u(x))| & \leq|f(x, 0)||h(x)|+\bar{C}|a(x)|(|u(x)|+|h(x)|)|h(x)| \\
& +\bar{C} \max \left(1,2^{\bar{\rho}}\right)\left(|u(x)|^{\bar{\rho}+1}+|h(x)|^{\bar{\rho}+1}\right)|h(x)| .
\end{aligned}
$$


Portanto

$$
\begin{aligned}
\int_{\Omega} \mid \widehat{F}(u+h) & -\widehat{F}(u)\left|d x=\int_{\Omega}\right| F(x, u(x)+h(x))-F(x, u(x)) \mid d x \\
& \leq \int_{\Omega}|f(x, 0)||h(x)| d x+\int_{\Omega} \bar{C}|a(x)|(|u(x)|+|h(x)|)|h(x)| d x \\
& +\int_{\Omega} \bar{C} \max \left(1,2^{\bar{\rho}}\right)\left(|u(x)|^{\bar{\rho}+1}+|h(x)|^{\bar{\rho}+1}\right)|h| d x \\
& \leq|\widehat{f}(0)|_{L^{2}(\Omega)}|h|_{L^{2}(\Omega)}+\bar{C}\left(|a u|_{L^{2}(\Omega)}+|a h|_{L^{2}(\Omega)}\right)|h|_{L^{2}(\Omega)} \\
& +\bar{C} \max \left(1,2^{\bar{\rho}+1}\right)\left(|u|_{L^{2(\bar{\rho}+1)}(\Omega)}^{\bar{\rho}+1}+|h|_{L^{2(\bar{\rho}+1)}(\Omega)}^{\bar{p}}\right)|h|_{L^{2}(\Omega)}
\end{aligned}
$$

o que conclui a demonstração da desigualdade (3.11). Finalmente, para demonstrar a desigualdade (3.12), notemos que a desigualdade (3.5) implica que para quase todo $x \in \Omega$

$$
\begin{aligned}
\mid F(x, u(x)+h(x)) & -F(x, u(x))-f(x, u(x) h(x) \mid \\
& \leq \bar{C}|a(x)||h(x)|^{2}+\bar{C} \max \left(1,2^{\bar{\rho}-1}\right)\left(|u(x)|^{\bar{\rho}}+|h(x)|^{\bar{\rho}}\right)|h(x)|^{2}
\end{aligned}
$$

e concluimos que

$$
\begin{aligned}
\int_{\Omega} \mid \widehat{F}(u+h) & -\widehat{F}(u)-\widehat{f}(u) h\left|d x=\int_{\Omega}\right| F(x, u(x)+h(x))-F(x, u(x))-f(x, u(x) h(x) \mid d x \\
& \leq \int_{\Omega} \bar{C}|(a h)(x)||h(x)| d x+\int_{\Omega} \bar{C} \max \left(1,2^{\bar{\rho}-1}\right)\left(|u(x)|^{\bar{\rho}}+|h(x)|^{\bar{\rho}}\right)|h(x)|^{2} d x
\end{aligned}
$$

Como

$$
\frac{\bar{\rho}}{2(\bar{\rho}+1)}+\frac{1}{2(\bar{\rho}+1)}+\frac{1}{2}=1
$$

segue do Lema 3.7 que

$$
\begin{aligned}
\mid \widehat{F}(u+h) & -\widehat{F}(u)-\left.\widehat{f}(u) h\right|_{L^{1}(\Omega)} \leq \bar{C}|a h|_{L^{2}(\Omega)}|h|_{L^{2}(\Omega)} \\
& +\bar{C} \max \left(1,2^{\bar{\rho}-1}\right)\left(|u|_{L^{2(\bar{\rho}+1)}(\Omega)}^{\bar{\rho}}+|h|_{L^{2(\bar{\rho}+1)}(\Omega)}^{\bar{\rho}}\right)|h|_{L^{2(\bar{\rho}+1)}(\Omega)}|h|_{L^{2}(\Omega)}
\end{aligned}
$$

Para completar a demonstração da proposição suponha que se $\bar{\rho}$ seja crítico (portanto $N \geq 3$ ) e sejam $r \in[N, \infty)$ e $u, h, v \in H_{0}^{1}(\Omega)$ arbitrários. A desigualdade (3.2) implica que

$$
\begin{aligned}
\mid\langle\widehat{f}(u+h) & -\widehat{f}(u), v\rangle_{L^{2}(\Omega)}\left|\leq \int_{\Omega}\right| f(x,(u+h)(x))-f(x, u(x))|v(x)| d x \\
& \leq \bar{C} \int_{\Omega}|(a h)(x)||v(x)| d x+\bar{C} \max \left(1,2^{\bar{\rho}-1}\right) \int_{\Omega}\left(|u(x)|^{\bar{\rho}}+|h(x)|^{\bar{\rho}}\right)|h(x)||| v(x) \mid d x .
\end{aligned}
$$


Como $a_{1} \in L^{r}(\Omega), h \in L^{2}(\Omega), v \in L^{2 r / r-2}(\Omega)$ e $\frac{1}{r}+\frac{1}{2}+\frac{r-2}{2 r}=1$, segue do Lema 3.7 que

$$
\int_{\Omega}\left|a_{1}(x) h(x) v(x)\right| d x \leq\left|a_{1}\right|_{L^{r}(\Omega)}|h|_{L^{2}(\Omega)}|v|_{L^{2 r / r-2}(\Omega)} .
$$

Além disso, como $a_{2} \in L^{\infty}(\Omega)$ e $h, v \in L^{2}(\Omega)$, aplicando a Desigualdade de Hölder obtemos

$$
\int_{\Omega}\left|a_{2}(x) h(x) v(x)\right| d x \leq\left|a_{2}\right|_{L^{\infty}(\Omega)} \int_{\Omega}|h(x) v(x)| d x \leq\left|a_{2}\right|_{L^{\infty}(\Omega)}|h|_{L^{2}(\Omega)}|v|_{L^{2}(\Omega)} .
$$

A igualdade

$$
\frac{1}{2^{*}}+\frac{1}{2}+\frac{1}{N}=1
$$

e o fato que $N \bar{\rho}=2^{*}$ implicam que $|u|^{\bar{\rho}} \in L^{N}(\Omega)$ e

$$
|u|_{L^{N}(\Omega)}^{\bar{\rho}}=\left[\int_{\Omega}\left(|u(x)|^{\bar{\rho}}\right)^{N}\right]^{1 / N}=\left[\int_{\Omega}|u(x)|^{2^{*} N}\right]^{\bar{\rho} / 2^{*}}=|u|_{L^{2^{*}}(\Omega)}^{\bar{\rho}} .
$$

Analogamente, mostramos que $|h|^{\bar{\rho}} \in L^{N}(\Omega)$ e $|h|_{L^{N}(\Omega)}^{\bar{\rho}}=|h|_{L^{2^{*}}(\Omega)}^{\bar{\rho}}$. Como $h \in L^{2}(\Omega)$ e $v \in$ $L^{2^{*}}(\Omega)$, aplicando novamente o Lema 3.7 obtemos

$$
\left.\int_{\Omega}\left(|u(x)|^{\bar{\rho}}+|h(x)|^{\bar{\rho}}\right)|h(x)||| v(x)\left|d x \leq\left(|u|_{L^{2^{*}}(\Omega)}^{\bar{\rho}}+|h|_{L^{2^{*}}(\Omega)}^{\bar{\rho}}\right)\right| h\right|_{L^{2}(\Omega)}|v|_{L^{2}(\Omega)} .
$$

Notemos agora que $r \leq N$ e $2 r /(r-2) \leq 2^{*}$. Seja $K \geq 0$ uma constante tal que para todo $w \in H_{0}^{1}(\Omega)$ temos

$$
\begin{aligned}
|w|_{L^{2}(\Omega)} & \leq K|w|_{H_{0}^{1}(\Omega)}, \\
|w|_{L^{2^{*}(\Omega)}} & \leq K|w|_{H_{0}^{1}(\Omega)}, \\
|w|_{L^{2 r /(r-2)}(\Omega)} & \leq K|w|_{H_{0}^{1}(\Omega)}
\end{aligned}
$$

Segue que

$$
\begin{aligned}
\left|\langle\widehat{f}(u+h)-\widehat{f}(u), v\rangle_{L^{2}(\Omega)}\right| \leq & \bar{C} K\left(\left|a_{1}\right|_{L^{r}(\Omega)}+\left|a_{2}\right|_{L^{\infty}(\Omega)}\right)|h|_{L^{2}(\Omega}|v|_{H^{1}(\Omega)}+ \\
& \bar{C} \max \left(1,2^{\bar{\rho}-1}\right) K\left(|u|_{L^{2^{*}(\Omega)}}^{\bar{\rho}}+|h|_{L^{2^{*}(\Omega)}}^{\bar{\rho}}\right)|h|_{L^{2}(\Omega)}|v|_{H^{1}(\Omega)}
\end{aligned}
$$

Como

$$
|\widehat{f}(u+h)-\widehat{f}(u)|_{H^{-1}(\Omega)}=\sup \left\{\left.\left|\langle\widehat{f}(u+h)-\widehat{f}(u), v\rangle_{L^{2}(\Omega)}\right|\left|v \in H_{0}^{1}(\Omega),\right| v\right|_{H_{0}^{1}(\Omega)} \leq 1\right\}
$$

segue o resultado. 
No restante do desenvolvimento do texto vamos assumir a seguinte hipótese:

Hipótese 3.9. Suponha válida a Hipótese 2.5 e a Hipótese 2.16 com um $\varepsilon \in(0, \infty)$ fixado. Além disso, seja $Y=L^{2}(\Omega) \times H^{-1}(\Omega)$.

Proposição 3.10. Seja $\bar{C}, \bar{\rho} \in[0, \infty)$ e $a: \Omega \rightarrow \mathbb{R}$ uma função mensurável tal que as aplicações

$$
u \mapsto \mid \text { a| и e } a \mapsto|a|^{1 / 2} u
$$

induzem operadores lineares limitados de $H_{0}^{1}(\Omega)$ em $L^{2}(\Omega)$. Suponha que a função $f: \Omega \times \mathbb{R} \rightarrow$ $\mathbb{R}$ satisfaz a condição $C^{1}$ Carathéodory e que $\partial_{u} f$ satisfaz a condição $(\bar{C}, \bar{\rho}, a)$ de crescimento. Além disso, suponha que $f(\cdot, 0) \in L^{2}(\Omega)$. Se $N \geq 3$, assuma também que $\bar{\rho} \leq\left(2^{*} / 2\right)-1$. Então

(i) f induz um operador de Nemytskii $\widehat{f}: H_{0}^{1}(\Omega) \rightarrow L^{2}(\Omega)$ que é Lipschitziano em subconjuntos limitados de $H_{0}^{1}(\Omega)$.

(ii) A primitiva canônica $F$ de $f$ induz um operador de Nemytskii $\widehat{F}: H_{0}^{1}(\Omega) \rightarrow L^{1}(\Omega)$. Além disso, $\widehat{F}$ é Frechet-diferenciável e

$$
D \widehat{F}(u)[h]=\widehat{f}(u) \cdot h, \text { para todo } u, h \in H_{0}^{1}(\Omega) \text {. }
$$

(iii) A aplicação $\Phi_{f}: Z \rightarrow Z$ dada por

$$
\Phi_{f}(z)=\left(0,(1 / \varepsilon) \widehat{f}\left(z_{1}\right)\right), z=\left(z_{1}, z_{2}\right) \in Z
$$

é limitada e Lipschitziana em subconjuntos limitados de $H_{0}^{1}(\Omega)$.

Demonstração. Mostremos que $\widehat{f}(u) \in L^{2}(\Omega)$ para todo $u \in H_{0}^{1}(\Omega)$. Seja $u \in H_{0}^{1}(\Omega)$. As hipóteses implicam que $|a| u \in L^{2}(\Omega)$ e $\widehat{f}(0) \in L^{2}(\Omega)$. Como $2 \leq 2(\bar{\rho}+1) \leq 2^{*}$, segue do Teorema 1.10 que $u \in L^{2(\bar{\rho}+1)}(\Omega)$. Uma aplicação da desigualdade $(3.8)$ da Proposição 3.8 implica que $\widehat{f}(u) \in L^{2}(\Omega)$.

Afirmamos que $\widehat{f}: H_{0}^{1}(\Omega) \rightarrow L^{2}(\Omega)$ é Lipschitziana em subconjuntos limitados de $H_{0}^{1}(\Omega)$. De fato, sejam $\widetilde{C}_{1}, \widetilde{C}_{2} \in(0, \infty)$ tais que

$$
\| a|u|_{L^{2}(\Omega)} \leq \widetilde{C}_{1}|u|_{H_{0}^{1}(\Omega)}, \text { para todo } u \in H_{0}^{1}(\Omega)
$$

e

$$
\left.\left.|| a\right|^{1 / 2} u\right|_{L^{2}(\Omega)} \leq \widetilde{C}_{2}|u|_{H_{0}^{1}(\Omega)}, \text { para todo } u \in H_{0}^{1}(\Omega)
$$


Sejam $R \geq 0$ e $u, v \in H_{0}^{1}(\Omega)$ com $|u|_{H_{0}^{1}(\Omega)} \leq R$ e $|v|_{H_{0}^{1}(\Omega)} \leq R$. A desigualdade (3.9) da Proposição 3.8, com $h=v-u$, implica que

$$
\begin{aligned}
& |\widehat{f}(v)-\widehat{f}(u)|_{L^{2}(\Omega)} \\
& \quad \leq \bar{C}|a(v-u)|_{L^{2}(\Omega)}+\bar{C} \max \left(1,2^{\bar{\rho}-1}\right)\left(|u|_{L^{2(\bar{\rho}+1)}(\Omega)}^{\overline{\bar{\rho}}}+|v-u|_{L^{2(\bar{\rho}+1)}(\Omega)}^{\overline{\bar{\rho}}}\right)|v-u|_{L^{2(\bar{\rho}+1)}(\Omega)}
\end{aligned}
$$

Como $2 \leq 2(\bar{\rho}+1) \leq 2^{*}$, segue do Teorema 1.10 que

$$
\begin{aligned}
\mid \widehat{f}(v)-\widehat{f} & \left.(u)\right|_{L^{2}(\Omega)} \leq \widetilde{C}_{2}|u-v|_{H_{0}^{1}(\Omega)} \\
& +\bar{C} \max \left(1,2^{\bar{\rho}-1}\right)\left(K_{2(\bar{\rho}+1)}^{\bar{\rho}+1}|u|_{H_{0}^{1}(\Omega)}^{\bar{\rho}}+K_{2(\bar{\rho}+1)}^{\bar{\rho}+1}|v-u|_{H_{0}^{1}(\Omega)}^{\bar{\rho}}\right) K_{2(\bar{\rho}+1)}|v-u|_{H_{0}^{1}(\Omega)} \\
& \leq \widetilde{C}_{2}|u-v|_{H_{0}^{1}(\Omega)}+\bar{C} \max \left(1,2^{\bar{\rho}-1}\right)\left(K_{2(\bar{\rho}+1)}^{\bar{\rho}+2} R^{\bar{\rho}}+K_{2(\bar{\rho}+1)}^{\bar{\rho}+2}\left(R^{\bar{\rho}}+R^{\bar{\rho}}\right)\right)|v-u|_{H_{0}^{1}(\Omega)} \\
& =\left(\widetilde{C}_{2}+3 \bar{C} \max \left(1,2^{\bar{\rho}-1}\right)\left(K_{2(\bar{\rho}+1)}^{\bar{\rho}+2} R^{\bar{\rho}}\right)|v-u|_{H_{0}^{1}(\Omega)}\right.
\end{aligned}
$$

e, portanto, a demonstração da afirmativa está concluída.

Passemos a demonstração de (ii). Notemos que para cada $u \in H_{0}^{1}(\Omega), a|u|^{2} \in L^{1}(\Omega)$. Como $\widehat{f}(0) \in L^{2}(\Omega)$ e $2 \leq 2(\bar{\rho}+1) \leq 2^{*}$, o Teorema 1.10 e a desigualdade (3.10) da Proposição 3.8 implicam que $\widehat{F}(u) \in L^{1}(\Omega)$.

Afirmamos que $\widehat{F}$ é Frechet diferenciável e $D \widehat{F}(u)[h]=\widehat{f}(u) \cdot h$. Sejam $u, h \in H_{0}^{1}(\Omega)$. Teorema 1.10 e a desigualdade (3.12) da Proposição 3.8 implicam que

$$
\begin{aligned}
\mid \widehat{F}(u+h) & -\widehat{F}(u)-\left.\widehat{f}(u) h\right|_{L^{1}(\Omega)} \\
& \leq\left(\bar{C}|a h|_{L^{2}(\Omega)}+\bar{C} \max \left(1,2^{\bar{\rho}-1}\right)\left(|u|_{L^{2(\bar{\rho}+1)}(\Omega)}^{\bar{\rho}}+|h|_{L^{2(\bar{\rho}+1)}(\Omega)}^{\bar{\rho}}\right)|h|_{L^{2(\bar{\rho}+1)}(\Omega)}\right)|h|_{L^{2}(\Omega)} \\
& \leq \bar{C} \widetilde{C}_{1}|h|_{H_{0}^{1}(\Omega)} K_{2}|h|_{H_{0}^{1}(\Omega)} \\
& +\bar{C} \max \left(1,2^{\bar{\rho}-1}\right)\left(K_{2(\bar{\rho}+1)}^{\bar{\rho}}|u|_{H_{0}^{1}(\Omega)}^{\bar{\rho}}+K_{2(\bar{\rho}+1)}^{\bar{\rho}}|h|_{H_{0}^{1}(\Omega)}^{\bar{\rho}}\right) K_{2(\bar{\rho}+1)}|h|_{H_{0}^{1}(\Omega)} K_{2}|h|_{H_{0}^{1}(\Omega)} \\
& =\bar{C} \widetilde{C}_{1} K_{2}|h|_{H_{0}^{1}(\Omega)}^{2} \\
& \left.+\bar{C} \max \left(1,2^{\bar{\rho}-1}\right)\left(K_{2(\bar{\rho}+1)}^{\bar{\rho}}|u|_{H_{0}^{1}(\Omega)}^{\bar{\rho}}+K_{2(\bar{\rho}+1)}^{\bar{\rho}}|h|_{H_{0}^{1}(\Omega)}^{\bar{\rho}}\right) K_{2(\bar{\rho}+1)}\right) K_{2}|h|_{H_{0}^{1}(\Omega)}^{2} .
\end{aligned}
$$

Logo,

$$
\frac{|\widehat{F}(u+h)-\widehat{F}(u)-\widehat{f}(u) h|_{L^{1}(\Omega)}}{|h|_{H_{0}^{1}(\Omega)}} \rightarrow 0, \text { quando }|h|_{H_{0}^{1}(\Omega)} \rightarrow 0
$$

e (ii) está demonstrada.

Finalmente para mostrar $(i i i)$, notemos que as propriedades de $\widehat{f}$ implicam que a aplicação $\Phi_{f}(z)=\left(0,(1 / \varepsilon) \widehat{f}\left(z_{1}\right)\right)$, para $\left(z_{1}, z_{2}\right) \in Z$, é limitada e Lipschitziana em subconjuntos limitados 
de $H_{0}^{1}(\Omega)$.

Observação 3.11. Segue da Proposição 2.7 que as hipóteses impostas na função a na Proposição 3.10 são satisfeitas se, por exemplo, $\widetilde{a} \in L_{u}^{p}\left(\mathbb{R}^{N}\right)$, com $p \geq N$.

Assuma a Hipótese 3.9 e as hipóteses do Proposição 3.10. A Proposição 2.17 implica que $B$ gera um $C_{0}$-semigrupo $\{T(t) \mid t \geq 0\}$ em $Z$ e a Proposição 3.1 implica que para cada $\zeta \in Z$, existe um $\omega_{\zeta}=\omega_{B, \Phi_{f}, \zeta} \in(0, \infty]$ e uma única aplicação contínua $z_{\zeta}:\left[0, \omega_{\zeta}\right) \rightarrow Z$ tal que

$$
z_{\zeta}(t)=T(t) \zeta+\int_{0}^{t} T(t-s) \Phi_{f}\left(z_{\zeta}(s)\right) d s, t \in\left[0, \omega_{\zeta}\right)
$$

Escrevendo $\zeta \pi_{f} t:=z_{\zeta}(t)$ para $t \in\left[0, \omega_{\zeta}\right)$, obtemos:

Teorema 3.12. $\pi_{f}$ é um semifluxo local em $Z$ e $\pi_{f}$ não explode em subconjuntos limitados de $H_{0}^{1}(\Omega)$.

Demonstração. O resultado segue da Proposição 3.1 .

O semifluxo local $\pi_{f}$ é o semifluxo gerado pelas soluções da equação da onda amortecida

$$
\begin{aligned}
\varepsilon u_{t t}+\alpha(x) u_{t}+\beta(x) u-\sum_{i, j} \partial_{i}\left(a_{i j}(x) \partial_{j} u\right) & =f(x, u), & x \in \Omega, t \in[0, \infty), \\
u(x, t) & =0, & x \in \partial \Omega, t \in[0, \infty) .
\end{aligned}
$$




\section{Capítulo}

\section{4}

\section{Existência de atrator global para o problema da onda amortecida definido em domínios} não limitados

Ao final do Capítulo 3, definimos o semifluxo local $\pi_{f}$ gerado pelas soluções da equação da onda amortecida

$$
\begin{aligned}
\varepsilon u_{t t}+\alpha(x) u_{t}+\beta(x) u-\sum_{i, j} \partial_{i}\left(a_{i j}(x) \partial_{j} u\right) & =f(x, u), & x \in \Omega, t \in[0, \infty), \\
u(x, t) & =0, & x \in \partial \Omega, t \in[0, \infty) .
\end{aligned}
$$

O objetivo deste capítulo é apresentar condições para que o semifluxo possua atrator global. As condições descritas na Hipótese 4.3 implicam que as condições da Proposição 3.10 estejam satisfeitas e fornecem estimativas de truncamento que nos auxiliam na verificação das hipóteses da Proposição 1.60 .

Na Seção 4.2 apresentamos as estimativas de truncamento, mostramos que $\pi_{f}$ é um semifluxo global e que as hipóteses $(b)$ e $(c)$ da Proposição 1.60 estão satisfeitas.

Já na Seção 4.3 novas condições serão necessárias para que o semifluxo $\pi_{f}$ seja assintoticamente compacto. Aqui teremos que distinguir o caso subcrítico (Hipótese 4.14) e o caso crítico (Hipótese 4.15). A compacidade assintótica é demonstrada no Teorema 4.17 (caso subcrítico) e no Teorema 4.19 (caso crítico), concluindo com a existência do atrator global.

Iniciamos o capítulo demonstrando um resultado auxiliar. 


\subsection{Um resultado preliminar}

O resultado a seguir será usado para justificar a diferenciação de funcionais definidos ao longo de soluções da equação de evolução semilinear apresentada no Capítulo 3 .

Teorema 4.1. Seja $Z$ um espaço de Banach e $\{T(t) \mid t \geq 0\}$ um $C_{0}$-semigrupo de operadores lineares em $Z$ com gerador infinitesimal $B: D(B) \subset Z \rightarrow Z$. Sejam $U \subset Z$ aberto, $Y$ espaço normado e $V: U \rightarrow Y$ uma função que, como aplicação de $Z$ em $Y$, é contínua em cada ponto de $U$ e Fréchet diferenciável em cada ponto de $U \cap D(B)$. Além disso, seja $W: U \times Z \rightarrow Y$ uma função contínua e tal que $D V(z)(B z+w)=W(z, w)$, para $z \in U \cap D(B)$ e $w \in Z$. Seja $\tau \in(0, \infty)$ $e I=[0, \tau]$. Considere $\bar{z} \in U, g: I \rightarrow Z$ contínua e $z$ uma aplicação de I em $U$ tal que, para $t \in I$,

$$
z(t)=T(t) \bar{z}+\int_{0}^{t} T(t-s) g(s) d s .
$$

Então, $V \circ z: I \rightarrow Y$ é diferenciável e

$$
(V \circ z)^{\prime}(t)=W(z(t), g(t)), \quad \text { para todo } t \in I
$$

Demonstração. Defina $|z|_{D(B)}:=|z|_{Z}+|B z|_{Z}, z \in D(B)$. Afirmamos que $\left(D(B),|\cdot|_{D(B)}\right)$ é um espaço de Banach. De fato, seja $\left(z_{n}\right)_{n}$ sequência de Cauchy em $\left(D(B),|\cdot|_{D(B)}\right)$. Mostremos que esta sequência é convergente em $\left(D(B),|\cdot|_{D(B)}\right)$. Para $n, m \in \mathbb{N}$ temos que

$$
\left|z_{n}-z_{m}\right|_{Z} \leq\left|z_{n}-z_{m}\right|_{D(B)} \text { e }\left|B z_{n}-B z_{m}\right|_{Z} \leq\left|z_{n}-z_{m}\right|_{D(B)}
$$

Logo, $\left(z_{n}\right)_{n}$ e $\left(B z_{n}\right)_{n}$ são sequências de Cauchy em $(Z,|\cdot| z)$. Como $(Z,|\cdot| z)$ é um espaço de Banach, existem $z, \widetilde{z} \in Z$ tais que $\left|z_{n}-z\right|_{Z} \rightarrow 0$ e $\left|B z_{n}-\tilde{z}\right|_{Z} \rightarrow 0$ quando $n \rightarrow \infty$. Por outro lado, como $B$ é um operador fechado, segue que $z \in D(B)$ e $B z=\widetilde{z}$. Como para todo $n \in \mathbb{N}$ temos

$$
\left|z_{n}-z\right|_{D(B)}=\left|z_{n}-z\right|_{Z}+\left|B z_{n}-B z\right|_{Z}
$$

segue que $\left|z_{n}-z\right|_{D(B)} \rightarrow 0$ quando $n \rightarrow \infty$. Portanto, concluímos que $\left(z_{n}\right)_{n}$ é uma sequência convergente em $\left(D(B),|\cdot|_{D(B)}\right)$.

$$
\begin{array}{r}
\text { Para } h \in(0, \infty) \text { e } t \in I, \text { defina } M_{h}=\sup _{t \in[0, h]}\|T(t)\|_{\mathscr{L}(Z)} \mathrm{e} \\
g_{h}(t)=\frac{1}{h} \int_{0}^{h} T(s) g(t) d s .
\end{array}
$$


Mostremos que $g_{h}(t) \in D(B)$ e $B g_{h}(t)=\frac{1}{h}(T(h) g(t)-g(t))$ para todo $h \in(0, \infty)$ e $t \in I$. De fato, fixado $t \in I$, defina $x_{h}:=g_{h}(t)$ e $x=g(t)$. Note que

$$
\begin{aligned}
\left|x_{h}-x\right|_{Z} & =\left|\frac{1}{h} \int_{0}^{h} T(\sigma) x d \sigma-x\right|_{Z}=\left|\frac{1}{h} \int_{0}^{h}(T(\sigma) x-x) d \sigma\right|_{Z} \\
& \leq \frac{1}{h} \int_{0}^{h}|T(\sigma) x-x|_{Z} d \sigma \leq \sup _{\sigma \in[0, h]}|T(\sigma) x-x|_{Z} .
\end{aligned}
$$

Logo, $x_{h} \rightarrow x$ quando $h \rightarrow 0^{+}$. Sabendo disso, para cada $h \in(0, \infty)$ fixado e $s<h$, temos que

$$
\begin{aligned}
& \frac{T(s) x_{h}-x_{h}}{s}=\frac{1}{s} \frac{1}{h} T(s) \int_{0}^{h} T(\sigma) x d \sigma-\frac{1}{s} \frac{1}{h} \int_{0}^{h} T(\sigma) x d \sigma \\
& =\frac{1}{s h} \int_{0}^{h} T(s+\sigma) x d \sigma-\frac{1}{s h} \int_{0}^{h} T(\sigma) x d \sigma=\frac{1}{s h} \int_{s}^{s+h} T(\sigma) x d \sigma-\frac{1}{s h} \int_{0}^{h} T(\sigma) x d \sigma \\
& =\frac{1}{s h} \int_{h}^{s+h} T(\sigma) x d \sigma-\frac{1}{s h} \int_{0}^{s} T(\sigma) x d \sigma=\frac{1}{s h} \int_{0}^{s} T(h+\sigma) x d \sigma-\frac{1}{s h} \int_{0}^{s} T(\sigma) x d \sigma \\
& =\frac{1}{s} \frac{1}{h} T(h) \int_{0}^{s} T(\sigma) x d \sigma-\frac{1}{s} \frac{1}{h} \int_{0}^{s} T(\sigma) x d \sigma=\frac{1}{h}(T(h)-I) \frac{1}{s} \int_{0}^{s} T(\sigma) x d \sigma
\end{aligned}
$$

e, portanto,

$$
\lim _{s \rightarrow 0^{+}} \frac{T(s) x_{h}-x_{h}}{s}=\lim _{s \rightarrow 0^{+}}\left(\frac{1}{h}(T(h)-I) \frac{1}{s} \int_{0}^{s} T(\sigma) x d \sigma\right)=\frac{1}{h}(T(h)-I) x .
$$

Logo, $g_{h}(t) \in D(B)$ e $B g_{h}(t)=\frac{1}{h}(T(h) g(t)-g(t))$. Sendo assim temos a função $g_{h}: I \rightarrow D(B)$ está bem definida. Além disso, para todo $t, t^{\prime} \in I$, a estimativa

$$
\begin{aligned}
\mid g_{h}(t) & -\left.g_{h}\left(t^{\prime}\right)\right|_{D(B)}=\left|\frac{1}{h} \int_{0}^{h} T(s)\left(g(t)-g\left(t^{\prime}\right)\right) d s\right|_{Z}+\mid \frac{1}{h}\left(T(h)\left(g(t)-g\left(t^{\prime}\right)\right)-\left.\left(g(t)-g\left(t^{\prime}\right)\right)\right|_{Z}\right. \\
& \leq\left.\frac{1}{h} \int_{0}^{h}\|T(s)\|\right|_{\mathscr{L}(Z)}\left|g(t)-g\left(t^{\prime}\right)\right|_{Z} d s+\frac{1}{h}\left(\|T(h)\| \mathscr{L}(Z)\left|g(t)-g\left(t^{\prime}\right)\right|_{Z}+\left|g(t)-g\left(t^{\prime}\right)\right|_{Z}\right) \\
& \leq M_{h}\left|g(t)-g\left(t^{\prime}\right)\right|_{Z}+\frac{1}{h}\left(M_{h}+1\right)\left|g(t)-g\left(t^{\prime}\right)\right|_{Z}
\end{aligned}
$$

e a continuidade da função $g$ mostram que $g_{h}$ é contínua em $I$. Mais ainda, afirmamos que $\left|g_{h}(t)-g(t)\right|_{Z} \rightarrow 0$ quando $h \rightarrow 0^{+}$, uniformemente em $I$. De fato, suponha que afirmativa não seja verdadeira. Logo existem $\varepsilon>0$ e sequências $\left(h_{m}\right)_{m}$ em $(0, \infty)$ e $\left(t_{m}\right)_{m}$ em $I$ tais que $h_{m} \rightarrow 0$, $t_{m} \rightarrow t \in I \mathrm{e}$

$$
\left|g_{h_{m}}\left(t_{m}\right)-g\left(t_{m}\right)\right|_{Z} \geq \varepsilon, \text { para todo } m \in \mathbb{N} \text {. }
$$


Porém, $\left|g_{h_{m}}\left(t_{m}\right)-g\left(t_{m}\right)\right|_{z} \leq\left|g_{h_{m}}\left(t_{m}\right)-g(t)\right|_{Z}+\left|g\left(t_{m}\right)-g(t)\right|_{z} \mathrm{e}$

$$
\begin{aligned}
\left|g_{h_{m}}\left(t_{m}\right)-g(t)\right|_{Z} & =\left|\frac{1}{h_{m}} \int_{0}^{h_{m}}\left(T(s) g\left(t_{m}\right)-g(t)\right) d s\right|_{Z} \\
& \leq\left|\frac{1}{h_{m}} \int_{0}^{h_{m}} T(s)\left(g\left(t_{m}\right)-g(t)\right) d s\right|_{Z}+\left|\frac{1}{h_{m}} \int_{0}^{h_{m}}(T(s) g(t)-g(t)) d s\right|_{Z} .
\end{aligned}
$$

Como $h_{m} \rightarrow 0$ quando $m \rightarrow \infty$, podemos assumir, tomando uma subsequência se necessário, que $h_{m} \leq 1$, para todo $m \in \mathbb{N}$. Logo,

$$
\left|\frac{1}{h_{m}} \int_{0}^{h_{m}} T(s)\left(g\left(t_{m}\right)-g(t)\right) d s\right|_{Z} \leq M_{1}\left|g\left(t_{m}\right)-g(t)\right|_{Z} \rightarrow 0, \text { quando } m \rightarrow \infty .
$$

Como $|T(s) g(t)-g(t)|_{Z} \rightarrow 0$ quando $s \rightarrow 0^{+}$, segue que

$$
\left|\frac{1}{h_{m}} \int_{0}^{h_{m}}(T(s) g(t)-g(t)) d s\right|_{Z} \rightarrow 0, \text { quando } m \rightarrow \infty .
$$

Assim, as fórmulas (4.2), (4.3) e (4.4) implicam que $\left|g_{h_{m}}\left(t_{m}\right)-g\left(t_{m}\right)\right|_{Z} \rightarrow 0$ quando $m \rightarrow \infty$, mas isso contradiz 4.1, o que prova a afirmação.

Seja $\bar{z} \in U$. Como o conjunto $D(B)$ é denso em $Z$, existe uma sequência $\left(\bar{z}_{m}\right)_{m}$ em $D(B)$ que converge para $\bar{z} \in Z$. Sendo $U \subset Z$ um conjunto aberto, podemos assumir, tomando uma subsequência se necessário, que $\bar{z}_{m} \in U \cap D(B)$, para todo $m \in \mathbb{N}$. Seja $\left(h_{m}\right)_{m}$ uma sequência em $(0, \infty)$ tal que $h_{m} \rightarrow 0$ quando $m \rightarrow \infty$. Para cada $m \in \mathbb{N}$ e $t \in I$, defina

$$
z_{m}(t)=T(t) \bar{z}_{m}+\int_{0}^{t} T(t-s) g_{h_{m}}(s) d s
$$

Afirmamos que $z_{m}(t) \in D(B)$ para todo $t \in I, z_{m}: I \rightarrow D(B)$ é contínua e diferenciável. De fato, temos que $g_{h_{m}}$ é contínua em $I$ com $g_{h_{m}}(t) \in D(B)$ para todo $t \in I$. Mostremos que $B g_{h_{m}}$ também é contínua em $I$. De fato, dados $t, t^{\prime} \in I$ temos

$$
\begin{aligned}
\left|B g_{h_{m}}(t)-B g_{h_{m}}\left(t^{\prime}\right)\right|_{Z} & =\left|\frac{1}{h_{m}}\left(T\left(h_{m}\right) g(t)-g(t)\right)-\frac{1}{h_{m}}\left(T\left(h_{m}\right) g\left(t^{\prime}\right)-g\left(t^{\prime}\right)\right)\right|_{Z} \\
& =\frac{1}{h_{m}}\left|\left(T\left(h_{m}\right) g(t)-g(t)\right)-\left(T\left(h_{m}\right) g\left(t^{\prime}\right)-g\left(t^{\prime}\right)\right)\right|_{Z} \\
& =\frac{1}{h_{m}}\left|T\left(h_{m}\right)\left(g(t)-g\left(t^{\prime}\right)\right)-\left(g(t)-g\left(t^{\prime}\right)\right)\right|_{Z} . \\
& \leq \frac{1}{h_{m}}\left\|T\left(h_{m}\right)\right\| \mathscr{L}(Z)\left|g(t)-g\left(t^{\prime}\right)\right|_{Z}+\frac{1}{h_{m}}\left|g(t)-g\left(t^{\prime}\right)\right|_{Z} \\
& \leq \frac{1}{h_{m}}\left(M_{h_{m}}+1\right)\left|g(t)-g\left(t^{\prime}\right)\right|_{Z} .
\end{aligned}
$$


A continuidade da função $g$ e a estimativa acima implicam a continuidade de $B g_{h_{m}}$. Logo, uma aplicação do Teorema 2.1 implica que $z_{m}(t)$ é solução do problema de Cauchy

$$
z_{m}^{\prime}(t)=B z_{m}+g_{h_{m}}(t), \text { e } z_{m}(0)=\bar{z}_{m}
$$

e a afirmativa é uma consequência do Teorema 2.1 .

Para concluir a demonstração do resultado, notemos que pelo o que já foi provado até agora, temos que $\left|z_{m}(t)-z(t)\right|_{Z} \rightarrow 0$ quando $m \rightarrow \infty$, uniformemente em $I$. De fato, sejam $m \in \mathbb{N}$ e $t \in I$. Temos

$$
\begin{aligned}
\left|z_{m}(t)-z(t)\right|_{Z} & =\left|T(t)\left(\bar{z}_{m}-\bar{z}\right)+\int_{0}^{t} T(t-s)\left(g_{h_{m}}(s)-g(s)\right) d s\right|_{Z} \\
& \leq M_{\tau}\left|\bar{z}_{m}-\bar{z}\right|_{Z}+\int_{0}^{t}\|T(t-s)\|_{\mathscr{L}(Z)}\left|g_{h_{m}}(s)-g(s)\right|_{Z} d s \\
& \leq M_{\tau}\left|\bar{z}_{m}-\bar{z}\right|_{Z}+M_{\tau} \tau \sup _{s \in[0, \tau]}\left|g_{h_{m}}(s)-g(s)\right|_{Z}
\end{aligned}
$$

o que implica que $\left|z_{m}(t)-z(t)\right|_{Z} \rightarrow 0$ quando $m \rightarrow \infty$, uniformemente em $I$. Logo, existe um $m_{0} \in \mathbb{N}$ tal que $z_{m}(t) \in U \cap D(B)$ para todo $m \geq m_{0}$ e todo $t \in I$. Além disso, as hipóteses sobre a aplicação $V$ e $W$ e o que já provamos implicam que $\left|\left(V \circ z_{m}\right)(t)-(V \circ z)(t)\right|_{Y} \rightarrow 0$ quando $m \rightarrow \infty$, uniformemente em $I$. Além disso, para cada $m \in \mathbb{N}$ e $t \in I$ temos

$$
\left(V \circ z_{m}\right)^{\prime}(t)=D V\left(z_{m}(t)\right)\left(B z_{m}(t)+g_{h_{m}}(t)\right)=W\left(z_{m}(t), g_{h_{m}}(t)\right) .
$$

Logo, $\left|W\left(z_{m}(t), g_{h_{m}}(t)\right)-W(z(t), g(t))\right|_{Y} \rightarrow 0$ quando $m \rightarrow \infty$, uniformemente em $I$. Portanto, $V \circ z$ é diferenciável em $Y$ e $(V \circ z)^{\prime}(t)=W(z(t), g(t))$, para $t \in I$. A demonstração está completa.

\subsection{Estimativas de truncamento}

Nesta seção mostraremos que a Hipótese 4.3, juntamente como as estimativas de truncamento (ver Teorema 4.9), implicam que o semifluxo $\pi_{f}$ é global. Também mostraremos que todo subconjunto limitado de $X$ é u-limitado e que existe um conjunto limitado $B_{0}$ em $X$ com a propriedade que para todo $x \in X$ existe um $t_{x} \in[0, \infty)$ tal que $x \pi t_{x} \in B_{0}$. Ou seja, provaremos que as hipóteses $(b)$ e $(c)$ da Proposição 1.60 são válidas. 
Proposição 4.2. Seja $\bar{\gamma}: \mathbb{R}^{N} \rightarrow[0,1]$ uma função de classe $C^{1}$ tal que

$$
\sup _{x \in \mathbb{R}^{N}}\left(|\bar{\gamma}(x)|^{2}+|\nabla \bar{\gamma}(x)|^{2}\right)<\infty
$$

Defina $\gamma=\bar{\gamma}^{2}$. Assuma as hipóteses e notações da Proposição 3.10 Fixe $\delta \in(0, \infty)$ e defina as funções $V=V_{\gamma}: Z \rightarrow \mathbb{R} e V^{*}=V_{\gamma}^{*}: Z \rightarrow \mathbb{R}$ por

$$
V(z)=\frac{1}{2} \int_{\Omega} \gamma(x) \Psi_{z}(x) d x \text { e } V^{*}(z)=\int_{\Omega} \gamma(x) F\left(x, z_{1}(x)\right) d x
$$

$\operatorname{para} z=\left(z_{1}, z_{2}\right) \in Z$, onde

$$
\Psi_{z}(x)=\varepsilon\left|\delta z_{1}(x)+z_{2}(x)\right|^{2}+\left(A \nabla z_{1}\right)(x) \cdot \nabla z_{1}(x)+\left(\beta(x)-\delta \alpha(x)+\delta^{2} \varepsilon\right)\left|z_{1}(x)\right|^{2}
$$

para $z=\left(z_{1}, z_{2}\right) \in Z$ e $x \in \Omega$. Sejam $\tau_{0} \in(0, \infty), I=\left[0, \tau_{0}\right]$ e $z: I \rightarrow Z$ uma solução de $\pi_{f}$. Então as funções $V \circ z e V^{*} \circ z$ são diferenciáveis e, para $t \in I$,

$$
\begin{aligned}
&(V \circ z)^{\prime}(t)=\int_{\Omega} \gamma(x)\left(\varepsilon\left(\delta z_{1}+z_{2}\right)\left(\delta z_{2}-(1 / \varepsilon) \alpha(x) z_{2}+(1 / \varepsilon) f\left(x, z_{1}(t)(x)\right)\right) d x\right. \\
&+\int_{\Omega} \gamma(x)\left(\left(-\delta \alpha(x)+\delta^{2} \varepsilon\right) z_{1} z_{2}-\delta \beta(x) z_{1} z_{2}\right) d x \\
&-\delta \int_{\Omega} \gamma(x)\left(A \nabla\left(z_{1}\right)\right) \cdot \nabla z_{1} d x-\int_{\Omega}\left(\delta z_{1}+z_{2}\right)(A \nabla \gamma) \cdot \nabla z_{1} d x \\
&\left(V^{*} \circ z\right)^{\prime}(t)=\int_{\Omega} \gamma(x) f\left(x, z_{1}(t)(x)\right) z_{2}(t)(x) d x
\end{aligned}
$$

$e$

$$
\begin{aligned}
(V \circ z)^{\prime}(t) & +2 \delta(V \circ z)(t)=\int_{\Omega} \gamma(x)(2 \delta \varepsilon-\alpha(x))\left(\delta z_{1}+z_{2}\right)^{2} d x \\
& +\int_{\Omega} \gamma(x)\left(\delta z_{1}+z_{2}\right) f\left(x, z_{1}(t)(x)\right) d x-\int_{\Omega}\left(\delta z_{1}+z_{2}\right)(A \nabla \gamma) \cdot \nabla z_{1} d x
\end{aligned}
$$

Demonstração. Notemos que as funções $V$ e $V^{*}$ estão bem definidas. Além disso, afirmamos que $V$ e $V^{*}$ são Fréchet-diferenciáveis em $Z$. De fato, considere as seguintes funções

$$
\begin{aligned}
& A(z)=(1 / 2) \int_{\Omega} \gamma(x) \varepsilon\left|\delta z_{1}(x)+z_{2}(x)\right|^{2} d x, \quad z=\left(z_{1}, z_{2}\right) \in Z \\
& B(z)=(1 / 2) \int_{\Omega} \gamma(x)\left(A \nabla z_{1}\right)(x) \cdot \nabla z_{1}(x) d x, \quad z=\left(z_{1}, z_{2}\right) \in Z \\
& C(z)=(1 / 2) \int_{\Omega} \gamma(x)\left(\beta(x)-\delta \alpha(x)+\delta^{2} \varepsilon\right)\left|z_{1}(x)\right|^{2} d x, \quad z=\left(z_{1}, z_{2}\right) \in Z
\end{aligned}
$$

Logo, $V(z)=A(z)+B(z)+C(z), z \in Z$. Notemos que as funções $A, B$ e $C$ são composições de 
funções Frechet-diferenciáveis em $Z$ e, portanto, a aplicação $V$ é Frechet-diferenciável em $Z$. Além disso,

$$
\begin{aligned}
D A(z)[\xi] & =\frac{1}{2} \int_{\Omega} \gamma(x) \varepsilon 2\left(\delta z_{1}(x)+z_{2}(x)\right)\left(\delta \xi_{1}(x)+\xi_{2}(x)\right) d x \\
& =\int_{\Omega} \gamma(x) \varepsilon\left(\delta z_{1}(x)+z_{2}(x)\right)\left(\delta \xi_{1}(x)+\xi_{2}(x)\right) d x \\
D B(z)[\xi] & =\frac{1}{2} \int_{\Omega} \gamma(x) 2\left(A \nabla z_{1}\right)(x) \cdot \nabla \xi_{1}(x) d x=\int_{\Omega} \gamma(x)\left(A \nabla z_{1}\right)(x) \cdot \nabla \xi_{1}(x) d x, \\
D C(z)[\xi] & =\frac{1}{2} \int_{\Omega} \gamma(x)\left(\beta(x)-\delta \alpha(x)+\delta^{2} \varepsilon\right) 2\left(z_{1}(x)\right) \xi_{1}(x) d x \\
& =\int_{\Omega} \gamma(x)\left(\beta(x)-\delta \alpha(x)+\delta^{2} \varepsilon\right)\left(z_{1}(x)\right) \xi_{1}(x) d x,
\end{aligned}
$$

onde $z=\left(z_{1}, z_{2}\right) \in Z$ e $\xi=\left(\xi_{1}, \xi_{2}\right) \in Z$. Obtemos que

$$
\begin{aligned}
D V(z)[\xi] & =\int_{\Omega} \gamma(x)\left(\varepsilon\left(\delta z_{1}(x)+z_{2}(x)\right)\left(\delta \xi_{1}(x)+\xi_{2}(x)\right)+\left(A(x) \nabla z_{1}(x)\right) \cdot \nabla \xi_{1}(x)\right) d x \\
& +\int_{\Omega} \gamma(x)\left(\left(\beta(x)-\delta \alpha(x)+\delta^{2} \varepsilon\right) z_{1}(x) \xi_{1}(x)\right) d x, z=\left(z_{1}, z_{2}\right) \in Z \text { e } \xi=\left(\xi_{1}, \xi_{2}\right) \in Z
\end{aligned}
$$

Analogamente, mostramos que $V^{*}$ é uma aplicação Frechet-diferenciável em $Z$ e

$$
D V^{*}(z)[\xi]=\int_{\Omega} \gamma(x) f\left(x, z_{1}(x)\right) \xi_{1}(x) d x, z=\left(z_{1}, z_{2}\right) \in Z \text { e } \xi=\left(\xi_{1}, \xi_{2}\right) \in Z
$$

Em particular, para $z=\left(z_{1}, z_{2}\right) \in D(B)$ e $w=\left(w_{1}, w_{2}\right) \in Z$, obtemos,

$$
\begin{gathered}
D V(z)[B z+w]=\int_{\Omega} \gamma(x) \varepsilon\left(\delta z_{1}+z_{2}\right) \delta\left(z_{2}+w_{1}\right) d x \\
+\int_{\Omega} \gamma(x) \varepsilon\left(\delta z_{1}+z_{2}\right)\left(-(1 / \varepsilon) \alpha(x) z_{2}+(1 / \varepsilon)\left(L z_{1}-\beta(x) z_{1}\right)+w_{2}\right) d x \\
+\int_{\Omega} \gamma(x)\left(\left(A \nabla z_{1}\right) \cdot \nabla\left(z_{2}+w_{1}\right)+\left(\beta(x)-\delta \alpha(x)+\delta^{2} \varepsilon\right) z_{1}\left(z_{2}+w_{1}\right)\right) d x \\
D V^{*}(z)[B z+w]=\int_{\Omega} \gamma(x) f\left(x, z_{1}\right)\left(z_{2}+w_{1}\right) d x .
\end{gathered}
$$

Observe que nas fórmulas acima omitimos o argumento $x$ em algumas das expressões. Notemos que

$$
\begin{aligned}
& D V(z)[B z+w]=\int_{\Omega} \gamma(x) \varepsilon\left(\delta z_{1}+z_{2}\right)\left(\delta\left(z_{2}+w_{1}\right)-(1 / \varepsilon) \alpha(x) z_{2}+w_{2}\right) d x \\
& \quad+\int_{\Omega} \gamma(x)\left(\delta z_{1}+z_{2}\right)\left(L z_{1}-\beta(x) z_{1}\right) d x \\
& \left.\quad+\int_{\Omega} \gamma(x)\left(\left(A \nabla z_{1}\right) \cdot \nabla w_{1}+\left(\beta(x)-\delta \alpha(x)+\delta^{2} \varepsilon\right) z_{1}\left(z_{2}+w_{1}\right)\right) d x+\int_{\Omega} \gamma(x)\left(A \nabla z_{1}\right) \cdot \nabla z_{2}\right) d x .
\end{aligned}
$$


A igualdade (2.11) implica que

$$
\begin{aligned}
\int_{\Omega} \gamma(x)\left(\delta z_{1}+z_{2}\right)\left(L z_{1}\right. & \left.-\beta(x) z_{1}\right) d x=-\int_{\Omega} A \nabla z_{1} \cdot \nabla\left(\gamma(x)\left(\delta z_{1}+z_{2}\right)\right) d x \\
& -\int_{\Omega} \beta(x) z_{1} \gamma(x)\left(\delta z_{1}+z_{2}\right) d x \\
& =-\int_{\Omega} \gamma(x) A \nabla\left(\delta z_{1}+z_{2}\right) \cdot \nabla z_{1} d x-\int_{\Omega}\left(\delta z_{1}+z_{2}\right)(A \nabla \gamma) \cdot \nabla z_{1} d x \\
& -\int_{\Omega} \gamma(x)\left(\delta z_{1}+z_{2}\right) \beta(x) z_{1} d x
\end{aligned}
$$

Além disso

$$
\begin{aligned}
-\int_{\Omega} \gamma(x) A \nabla\left(\delta z_{1}+z_{2}\right) \cdot \nabla z_{1} d x & \left.-\int_{\Omega}\left(\delta z_{1}+z_{2}\right)(A \nabla \gamma) \cdot \nabla z_{1} d x+\int_{\Omega} \gamma(x)\left(A \nabla z_{1}\right) \cdot \nabla z_{2}\right) d x \\
& =-\int_{\Omega} \gamma(x)\left(A \nabla\left(\delta z_{1}\right)\right) \cdot \nabla z_{1} d x-\int_{\Omega}\left(\delta z_{1}+z_{2}\right)(A \nabla \gamma) \cdot \nabla z_{1} d x
\end{aligned}
$$

Portanto,

$$
\begin{aligned}
D V(z)[B z & +w]=\int_{\Omega} \gamma(x) \varepsilon\left(\delta z_{1}+z_{2}\right)\left(\delta\left(z_{2}+w_{1}\right)-(1 / \varepsilon) \alpha(x) z_{2}+w_{2}\right) d x \\
& +\int_{\Omega} \gamma(x)\left(\left(A \nabla z_{1}\right) \cdot \nabla w_{1}+\left(-\delta \alpha(x)+\delta^{2} \varepsilon\right) z_{1}\left(z_{2}+w_{1}\right)+\beta(x)\left(z_{1} w_{1}-\delta z_{1} z_{1}\right)\right) d x \\
& -\int_{\Omega} \gamma(x)\left(A \nabla\left(\delta z_{1}\right)\right) \cdot \nabla z_{1} d x-\int_{\Omega}\left(\delta z_{1}+z_{2}\right)(A \nabla \gamma) \cdot \nabla z_{1} d x
\end{aligned}
$$

Defina agora as aplicações $W: Z \times Z \rightarrow \mathbb{R}$ e $W^{*}: Z \times Z \rightarrow \mathbb{R}$ por

$$
\begin{aligned}
W(z, w) & =\int_{\Omega} \gamma(x)\left(\varepsilon\left(\delta z_{1}+z_{2}\right)\left(\delta\left(z_{2}+w_{1}\right)+\left(-(1 / \varepsilon) \alpha(x) z_{2}+w_{2}\right)\right) d x\right. \\
& \left.+\int_{\Omega} \gamma(x)\left(\left(A \nabla z_{1}\right) \cdot \nabla w_{1}+\left(-\delta \alpha(x)+\delta^{2} \varepsilon\right) z_{1}\left(z_{2}+w_{1}\right)\right)+\beta(x)\left(z_{1} w_{1}-\delta z_{1} z_{1}\right)\right) d x \\
& -\int_{\Omega} \gamma(x)\left(A \nabla\left(\delta z_{1}\right)\right) \cdot \nabla z_{1} d x-\int_{\Omega}\left(\delta z_{1}+z_{2}\right)(A \nabla \gamma) \cdot \nabla z_{1} d x, \\
W^{*}(z, w) & =\int_{\Omega} \gamma(x) f\left(x, z_{1}\right)\left(z_{2}+w_{1}\right) d x
\end{aligned}
$$

para $(z, w) \in Z \times Z \operatorname{com} z=\left(z_{1}, z_{2}\right)$ e $w=\left(w_{1}, w_{2}\right)$. Em particular para $w_{1}=0$, obtemos

$$
\begin{aligned}
W(z, w) & =\int_{\Omega} \gamma(x)\left(\varepsilon\left(\delta z_{1}+z_{2}\right)\left(\delta z_{2}+\left(-(1 / \varepsilon) \alpha(x) z_{2}+w_{2}\right)\right)\right. \\
& +\int_{\Omega} \gamma(x)\left(\left(-\delta \alpha(x)+\delta^{2} \varepsilon\right) z_{1} z_{2}-\delta \beta(x) z_{1} z_{1}\right) d x \\
& -\int_{\Omega} \gamma(x)\left(A \nabla\left(\delta z_{1}\right)\right) \cdot \nabla z_{1} d x-\int_{\Omega}\left(\delta z_{1}+z_{2}\right)(A \nabla \gamma) \cdot \nabla z_{1} d x \\
W^{*}(z, w) & =\int_{\Omega} \gamma(x) f\left(x, z_{1}\right) z_{2} d x .
\end{aligned}
$$


Afirmamos que as aplicações $W$ e $W^{*}$ são contínuas de $Z \times Z$. De fato, sejam $(z, w),(\widetilde{z}, \widetilde{w}) \in$ $Z \times Z$. Temos que

$$
\begin{aligned}
\left|W^{*}(z, w)-W^{*}(\widetilde{z}, \widetilde{w})\right| & \leq \int_{\Omega} \gamma(x)\left|f\left(x, z_{1}(x)\right) z_{2}-f\left(x, \widetilde{z}_{1}(x)\right) \widetilde{z}_{2}\right| d x \\
& \leq \int_{\Omega} \gamma(x)\left|f\left(x, z_{1}(x)\right)-f\left(x, \widetilde{z}_{1}(x)\right)\right|\left|z_{2}+w_{1}\right| d x \\
& +\int_{\Omega} \gamma(x)\left|f\left(x, \widetilde{z}_{1}(x)\right)\right| \mid\left(z_{2}-\widetilde{z}_{2}\right)+\left(w_{1}-\widetilde{w_{1}} \mid d x\right.
\end{aligned}
$$

onde $z=\left(z_{1}, z_{2}\right), \widetilde{z}=\left(\widetilde{z}_{1}, \widetilde{z}_{2}\right), w=\left(w_{1}, w_{2}\right)$ e $\widetilde{w}=\left(\widetilde{w}_{1}, \widetilde{w}_{2}\right)$. A hipótese imposta na função $\gamma$ e a Proposição 3.6 implicam a continuidade da aplicação $W^{*}$. Analogamente, utilizando a Hipótese 2.5 e a Proposição 3.8, mostramos a continuidade da aplicação $W$. A afirmativa está demonstrada.

Sejam $\tau_{0} \in(0, \infty), I=\left[0, \tau_{0}\right]$ e $z: I \rightarrow Z$ uma solução de $\pi_{f}$. O Teorema 4.1 e as fórmulas (4.8) e 4.9) implicam as igualdades (4.5) e 4.6). Para concluir a demonstração da proposição, verifiquemos que a igualdade em (4.7) é válida. De fato,

$$
\begin{aligned}
(V \circ z)^{\prime}( & t)+2 \delta(V \circ z)(t)=\int_{\Omega} \gamma(x)\left(\varepsilon ( \delta z _ { 1 } + z _ { 2 } ) \left(\delta z_{2}+\left(-(1 / \varepsilon) \alpha(x) z_{2}+(1 / \varepsilon) f\left(x, z_{1}\right)\right) d x\right.\right. \\
& \left.+\int_{\Omega} \gamma(x)\left(\left(-\delta \alpha(x)+\delta^{2} \varepsilon\right) z_{1} z_{2}-\delta \beta(x) z_{1} z_{1}\right)\right) d x-\int_{\Omega} \gamma(x) \delta\left(A \nabla z_{1}\right) \cdot \nabla z_{1} d x \\
& -\int_{\Omega}\left(\delta z_{1}+z_{2}\right)(A \nabla \gamma) \cdot \nabla z_{1} d x \\
& +2 \delta \frac{1}{2} \int_{\Omega} \gamma(x) \varepsilon\left|\delta z_{1}+z_{2}\right|^{2}+\left(A \nabla z_{1}\right) \cdot \nabla z_{1}+\left(\beta(x)-\delta \alpha(x)+\delta^{2} \varepsilon\right)\left|z_{1}\right|^{2} d x \\
& =\int_{\Omega} \gamma(x)\left(\delta z_{1}+z_{2}\right) f\left(x, z_{1}(t)(x)\right) d x-\int_{\Omega}\left(\delta z_{1}+z_{2}\right)(A \nabla \gamma) \cdot \nabla z_{1} d x \\
& +\int_{\Omega} \gamma(x) \delta(\varepsilon \delta-\alpha(x)) z_{1} z_{2} d x+\int_{\Omega} \gamma(x)(\varepsilon \delta-\alpha(x))\left|z_{2}\right|^{2} d x \\
& +\delta \int_{\Omega} \gamma(x)(-\alpha(x)+\delta \varepsilon) z_{1} z_{2} d x-\int_{\Omega} \gamma(x) \delta \beta(x)\left|z_{1}\right|^{2} d x \\
& -\delta \int_{\Omega} \gamma(x)\left(A \nabla z_{1}\right) \cdot \nabla z_{1} d x+\delta \int_{\Omega} \gamma(x) \varepsilon\left|\delta z_{1}+z_{2}\right|^{2} d x \\
& +\delta \int_{\Omega} \gamma(x)\left(A \nabla z_{1}\right) \cdot \nabla z_{1} d x+\delta \int_{\Omega} \gamma(x) \beta(x)\left|z_{1}\right|^{2} d x+\delta^{2} \int_{\Omega} \gamma(x)(-\alpha(x)+\delta \varepsilon)\left|z_{1}\right|^{2} d x \\
& =\int_{\Omega} \gamma(x)\left(\delta z_{1}+z_{2}\right) f\left(x, z_{1}(t)(x)\right) d x-\int_{\Omega}\left(\delta z_{1}+z_{2}\right)(A \nabla \gamma) \cdot \nabla z_{1} d x \\
& +\int_{\Omega} \gamma(x)(\varepsilon \delta-\alpha(x))\left(\left|\delta z_{1}\right|^{2}+2 \delta z_{1} z_{2}+\left|z_{2}\right|^{2}\right) d x+\varepsilon \delta \int_{\Omega} \gamma(x)\left(\delta z_{1}+z_{2}\right)^{2} d x \\
& =\int_{\Omega} \gamma(x)\left(\delta z_{1}+z_{2}\right) f\left(x, z_{1}(t)(x)\right) d x-\int_{\Omega}\left(\delta z_{1}+z_{2}\right)(A \nabla \gamma) \cdot \nabla z_{1} d x \\
& +\int_{\Omega} \gamma(x)\left(\delta z_{1}+z_{2}\right)^{2}(2 \varepsilon \delta-\alpha(x)) d x .
\end{aligned}
$$


A demonstração está completa.

Considere as seguintes hipóteses adicionais:

Hipótese 4.3. (a) $\alpha_{0}>0$;

(b) $\bar{C}, \bar{\rho}, \bar{\tau} \in[0, \infty)$ e $\bar{\mu} \in(0, \infty)$ são constantes e c $: \Omega \rightarrow \mathbb{R}$ é uma função com $c \in L^{1}(\Omega)$. Se $N \geq 3$, então $\bar{\rho} \leq\left(2^{*} / 2\right)-1$;

(c) $a: \Omega \rightarrow \mathbb{R}$ é uma função mensurável tal que $u \mapsto|a|$ e e $u \mapsto|a|^{1 / 2} u$ induzem operadores lineares limitados de $H_{0}^{1}(\Omega)$ em $L^{2}(\Omega)$;

(d) $f: \Omega \times \mathbb{R} \rightarrow \mathbb{R}$ satisfaz a condição $C^{1}$-Carathéodory;

(e) F é a primitiva canônica de $f$;

(f) $\partial_{u} f$ satisfaz a condição de $(\bar{C}, \bar{\rho}, a)$ de crescimento;

(g) $|f(\cdot, 0)|_{L^{2}(\Omega)} \leq \bar{\tau}$

(h) $f(x, u) u-\bar{\mu} F(x, u) \leq c(x)$ e $F(x, u) \leq c(x)$ para quase todo $x \in \Omega$ e para todo $u \in \mathbb{R}$.

A condição (h) na Hipótese 4.3 é uma condição de dissipatividade que implicará a existência de atratores globais para o problema estudado. O próximo lema apresenta uma condição suficiente para a hipótese de dissipatividade $(h)$.

Na demonstração do lema a seguir utilizaremos o seguinte fato:

Observação 4.4. Seja $h: \mathbb{R} \rightarrow \mathbb{R}$ uma função contínua e não crescente. Então

$$
h(u) u \leq \int_{0}^{u} h(s) d s, \text { para todo } u \in \mathbb{R} .
$$

Lema 4.5. Seja $f: \Omega \times \mathbb{R} \rightarrow \mathbb{R}$ uma função satisfazendo a condição $C^{0}$ Carathéodory e seja $F$ a canônica primitiva de $f$. Sejam $v, \gamma \in(1, \infty)$ constantes e $D \in L^{1}(\Omega)$ uma função com $D(x)>0$ para todo $x \in \Omega$ e suponha que

$$
F(x, u) \leq D(x) \text { para todo } x \in \Omega \text { e todo } u \in \mathbb{R}
$$

Assuma também que a função $u \mapsto(\gamma D(x)-F(x, u))^{v}$ seja convexa para quase todo $x \in \Omega$. Defina

$$
\bar{\mu}=(1 / v) \text { e } c(x)=\max \left(1, \gamma^{v}(\gamma-1)^{1-v} v^{-1}\right) D(x), x \in \Omega
$$

Então $f(x, u) u-\bar{\mu} F(x, u) \leq c(x)$ e $F(x, u) \leq c(x)$ para quase todo $x \in \Omega$ e todo $u \in \mathbb{R}$. 
Demonstração. Defina $G(x, u)=-(\gamma D(x)-F(x, u))^{v}$ para $x \in \Omega$ e $u \in \mathbb{R}$. A convexidade da função $u \mapsto(\gamma D(x)-F(x, u))^{v}$ implica que $u \mapsto \partial_{u} G(x, u)=v f(x, u)(\gamma D(x)-F(x, u))^{v-1}$ é uma função não-crescente e contínua para quase todo $x \in \Omega$. A desigualdade da Observação 4.4 implica que para quase todo $x \in \Omega$ e todo $u \in \mathbb{R}$,

$$
v f(x, u)(\gamma D(x)-F(x, u))^{v-1} u \leq G(x, u)-G(x, 0)=-(\gamma D(x)-F(x, u))^{v}+(\gamma D(x))^{v} .
$$

Como $F(x, u) \leq D(x)$, segue que

$$
F(x, u) \leq \max \left(1, \gamma^{v}(\gamma-1)^{1-v} v^{-1}\right) D(x) \text { para quase todo } x \in \Omega \text { e todo } u \in \mathbb{R} \text {. }
$$

Além disso, para quase todo $x \in \Omega$ e todo $u \in \mathbb{R}$, temos $\gamma D(x)-F(x, u) \geq(\gamma-1) D(x)>0$ e

$$
\begin{aligned}
v f(x, u) u & \leq-(\gamma D(x)-F(x, u))+(\gamma D(x))^{v}((\gamma-1) D(x))^{1-v} \\
& \leq F(x, u)+\gamma^{v}(\gamma-1)^{1-v} D(x) .
\end{aligned}
$$

Portanto, para quase todo $x \in \Omega$ e todo $u \in \mathbb{R}$ temos

$$
f(x, u) u-v^{-1} F(x, u) \leq \gamma^{v}(\gamma-1)^{1-v} v^{-1} D(x),
$$

o que conclui a demonstração.

Observação 4.6. Uma função $f: \Omega \times \mathbb{R} \rightarrow \mathbb{R}$ tal que sua canônica primitiva de $F$ seja $F(x, u)=$ $-D(x) e^{-u}, x \in \Omega$ e $u \in \mathbb{R}$, onde $D \in L^{1}(\Omega)$ com $D(x)>0$ para todo $x \in \Omega$, satisfaz as condições do Lema 4.5. Outro exemplo, é $F(x, u)=-|u|^{2 r}+D(x), x \in \Omega$ e $u \in \mathbb{R}$, onde $0 \leq r \leq(\bar{\rho}+2) / 2$ e $D \in L^{1}(\Omega) \operatorname{com} D(x)>0$ para todo $x \in \Omega$.

Fixe uma função $\bar{\vartheta}: \mathbb{R} \rightarrow[0,1]$ de classe $C^{\infty} \operatorname{com} \bar{\vartheta}(s)=0$ se $s \in(-\infty, 1]$ e $\bar{\vartheta}(s)=1$ para $s \in[2, \infty)$. Defina $\vartheta:=\bar{\vartheta}^{2}$. Para $k \in \mathbb{N}$, sejam $\bar{\vartheta}_{k}: \mathbb{R}^{N} \rightarrow \mathbb{R}$ e $\vartheta_{k}: \mathbb{R}^{N} \rightarrow \mathbb{R}$ as funções definidas por

$$
\bar{\vartheta}_{k}(x)=\bar{\vartheta}\left(|x|^{2} / k^{2}\right) \text { e } \vartheta_{k}(x)=\vartheta\left(|x|^{2} / k^{2}\right), x \in \mathbb{R}^{N}
$$

Observação 4.7. Segue que para cada $k \in \mathbb{N}$, as funções $\vartheta_{k} e \bar{\vartheta}_{k}$ são nulas na bola fechada de centro na origem e raio $k$ e constante igual a 1 fora da bola fechada de centro na origem e raio $\sqrt{2} k$.

A propriedade da diferenciação do produto de funções em $H^{1}(\Omega)$ (ver Proposição 1.6) implica que

Lema 4.8. Para todo $u \in H^{1}(\Omega) \cap L^{\infty}(\Omega), \bar{\gamma} u \in H^{1}(\Omega) \cap L^{\infty}(\Omega) e \nabla(\bar{\gamma} u)=\bar{\gamma}(\nabla u)+(\nabla \bar{\gamma}) u$. 
O próximo resultado apresenta as estimativas de truncamento que auxilirão na demonstração do Teorema 4.12 ,

Teorema 4.9. Assuma a Hipótese 3.9 e a Hipótese 4.3 Escolha $\delta$ e v em $(0, \infty)$ com

$$
v \leq \min (1, \bar{\mu} / 2), \lambda_{1}-\delta \alpha_{1}>0 \text { e } \alpha_{0}-2 \delta \varepsilon \geq 0
$$

Então existe uma constante $c^{\prime} \in[0, \infty)$ e para todo $R \in[0, \infty)$, existe uma constante $M^{\prime}=M_{R}^{\prime}$ tal que para todo $\tau_{0} \in[0, \infty)$ e toda solução $z(\cdot)$ de $\pi_{f}$ em $I=\left[0, \tau_{0}\right]$ com $|z(0)|_{z} \leq R$ temos

$$
\begin{aligned}
\int_{\Omega}\left((\varepsilon / 2)\left|z_{2}(t)(x)\right|^{2}\right. & +\left(A(x) \nabla z_{1}(t)(x)\right) \cdot \nabla z_{1}(t)(x) \\
+ & \left.(\beta(x)-\delta \alpha(x))\left|z_{1}(t)(x)\right|^{2}\right) d x \leq c^{\prime}+M^{\prime} e^{-2 \delta v t}, \text { para } t \in I .
\end{aligned}
$$

Se $|z(t)|_{z} \leq R$, para todo $t \in I$, então para cada $k \in \mathbb{N}$ existe uma constante $c_{k}=c_{k, R} \in[0, \infty)$ tal que $c_{k} \rightarrow 0$ quando $k \rightarrow \infty e$

$$
\begin{aligned}
\int_{\Omega} \vartheta_{k}(x) & \left((\varepsilon / 2)\left|z_{2}(t)(x)\right|^{2}+\left(A(x) \nabla z_{1}(t)(x)\right) \cdot \nabla z_{1}(t)(x)\right. \\
& \left.+(\beta(x)-\delta \alpha(x))\left|z_{1}(t)(x)\right|^{2}\right) d x \leq c_{k}+M^{\prime} e^{-2 \delta v t}, \text { para } k \in \mathbb{N} e t \in I
\end{aligned}
$$

Para demonstrar o Teorema 4.9 necessitamos do seguinte lema auxiliar.

Lema 4.10. Assuma as hipóteses do Teorema 4.9. Sejam $\bar{\gamma}, \gamma, V=V_{\gamma}$ e $V^{*}=V_{\gamma}^{*}$ como na Proposição 4.2 Para todo $z \in Z$, defina $s(z)=s_{\bar{\gamma}}(z)$ por

$$
s(z)(x)=-2 \bar{\gamma}(x) z_{1}(x)(A(x) \nabla \bar{\gamma}(x)) \cdot \nabla z_{1}(x)-\left|z_{1}(x)\right|^{2}(A(x) \nabla \bar{\gamma}(x)) \cdot \nabla \bar{\gamma}(x), x \in \Omega .
$$

Dado $\tau_{0} \in[0, \infty)$ e dada uma solução $z(\cdot)$ de $\pi_{f}$ definida em $I=\left[0, \tau_{0}\right]$, considere

$$
\eta(t)=\eta_{\gamma}(t)=V(z(t))-V^{*}(z(t)), t \in I .
$$

Então, para $t \in I$,

$$
\begin{aligned}
\eta^{\prime}(t)+2 \delta v \eta(t) & \leq \delta(\bar{\mu}-2 v+1) \int_{\Omega} \gamma(x) c(x) d x \\
& -\int_{\Omega}\left(\delta z_{1}+z_{2}\right)(A \nabla \gamma) \cdot \nabla z_{1} d x-\delta(1-v) \int_{\Omega} s(z(t))(x) d x
\end{aligned}
$$


Demonstração. Segue Lema 4.8 que para todo $z=\left(z_{1}, z_{2}\right) \in Z$ e $x \in \Omega$

$$
\begin{aligned}
\left(A(x) \nabla\left(\bar{\gamma} z_{1}\right)(x)\right) \cdot \nabla\left(\bar{\gamma} z_{1}\right)(x) & =|\bar{\gamma}(x)|^{2}\left(A(x) \nabla z_{1}(x)\right) \cdot \nabla z_{1}(x) \\
& +2 \bar{\gamma}(x) z_{1}(x)(A(x) \nabla \bar{\gamma}(x)) \cdot \nabla z_{1}(x)+\left|z_{1}(x)\right|^{2}(A(x) \nabla \bar{\gamma}(x)) \cdot \nabla \bar{\gamma}(x) \\
& =|\bar{\gamma}(x)|^{2}\left(A(x) \nabla z_{1}(x)\right) \cdot \nabla z_{1}(x)-s(z)(x) .
\end{aligned}
$$

Esta igualdade e a definição de $V$ implicam para todo $z=\left(z_{1}, z_{2}\right) \in Z$ que

$$
\begin{aligned}
2 V(z) & =\int_{\Omega} \gamma(x)\left(\varepsilon\left|\delta z_{1}(x)+z_{2}(x)\right|^{2}+\left(A \nabla z_{1}\right)(x) \cdot \nabla z_{1}(x)+\left(\beta(x)-\delta \alpha(x)+\delta^{2} \varepsilon\right)\left|z_{1}(x)\right|^{2}\right) d x \\
& \geq \int_{\Omega} \gamma(x)\left(\left(A(x) \nabla z_{1}(x)\right) \cdot \nabla z_{1}(x)+(\beta(x)-\delta \alpha(x))\left|z_{1}(x)\right|^{2}\right) d x \\
& =\int_{\Omega}\left(\left(A(x) \nabla\left(\bar{\gamma} z_{1}\right)(x) \cdot \nabla\left(\bar{\gamma} z_{1}\right)(x)+\int_{\Omega} \beta(x)\left|\left(\bar{\gamma} z_{1}\right)(x)\right|^{2} d x-\int_{\Omega} \delta \alpha(x)\left|\left(\bar{\gamma} z_{1}\right)(x)\right|^{2} d x\right.\right. \\
& +\int_{\Omega} s(z)(x) d x \geq\left(\lambda_{1}-\delta \alpha_{1}\right)\left|\bar{\gamma} z_{1}\right|_{L^{2}(\Omega)}^{2}+\int_{\Omega} s(z)(x) d x .
\end{aligned}
$$

Como $\left(\lambda_{1}-\delta \alpha_{1}\right)>0$, temos que

$$
2 V(z) \geq \int_{\Omega} s(z)(x) d x, \quad \text { para todo } z \in Z
$$

Seja $\tau_{0} \in[0, \infty)$ e seja $z(\cdot)=\left(z_{1}(\cdot), z_{2}(\cdot)\right)$ uma solução de $\pi_{f}$ definida em $I=\left[0, \tau_{0}\right]$. A desigualdade 4.14) implica que

$$
\begin{aligned}
(V \circ z)^{\prime}(t)+2 \delta v(V \circ z)(t) & =(V \circ z)^{\prime}(t)+2 \delta v(V \circ z)(t)+2 \delta(V \circ z)(t)-2 \delta(V \circ z)(t) \\
& =(V \circ z)^{\prime}(t)+2 \delta(V \circ z)(t)-\delta(1-v) 2(V \circ z)(t) \\
& \leq(V \circ z)^{\prime}(t)+2 \delta(V \circ z)(t)-\delta(1-v) \int_{\Omega} s(z(t))(x) d x .
\end{aligned}
$$

Como $2 \delta \varepsilon-\alpha_{0}<0$, segue que

$$
\begin{aligned}
(V \circ z)^{\prime}(t) & +2 \delta v(V \circ z)(t)+\delta(1-v) \int_{\Omega} s(z(t))(x) d x \leq(V \circ z)^{\prime}(t)+2 \delta(V \circ z)(t) \\
& \leq\left(2 \delta \varepsilon-\alpha_{0}\right) \int_{\Omega} \gamma(x)\left(\delta z_{1}(t)+z_{2}(t)\right)^{2} d x \\
& +\int_{\Omega} \gamma(x)\left(\delta z_{1}(t)+z_{2}(t)\right) f\left(x, z_{1}(t)(x)\right) d x-\int_{\Omega}\left(\delta z_{1}(t)+z_{2}(t)\right)(A \nabla \gamma) \cdot \nabla z_{1}(t) d x \\
& \leq \delta \int_{\Omega} \gamma(x) z_{1}(t) f\left(x, z_{1}(t)(x)\right) d x+\int_{\Omega} \gamma(x) z_{2}(t) f\left(x, z_{1}(t)(x)\right) d x \\
& -\int_{\Omega}\left(\delta z_{1}(t)+z_{2}(t)\right)(A \nabla \gamma) \cdot \nabla z_{1} d x
\end{aligned}
$$


e temos

$$
\begin{aligned}
(V \circ z)^{\prime}(t) & +2 \delta v(V \circ z)(t)+\delta(1-v) \int_{\Omega} s(z(t))(x) d x \\
& \leq \delta \int_{\Omega} \gamma(x)\left(\bar{\mu} F\left(x, z_{1}(t)(x)\right)+c(x)\right) d x-2 \delta v\left(V^{*} \circ z\right)(t) \\
& +2 \delta v\left(V^{*} \circ z\right)(t)+\left(V^{*} \circ z\right)^{\prime}(t)-\int_{\Omega}\left(\delta z_{1}(t)+z_{2}(t)\right)(A \nabla \gamma) \cdot \nabla z_{1}(t) d x=: S^{*}
\end{aligned}
$$

Ou seja, obtemos que

$$
(V \circ z)^{\prime}(t)+2 \delta v(V \circ z)(t)+\delta(1-v) \int_{\Omega} s(z(t))(x) d x \leq S^{*}, \text { para todo } t \in I .
$$

Logo para concluir a demonstração da desigualdade (4.13), basta mostrar que

$$
\begin{aligned}
S^{*} & \leq \delta(\bar{\mu}-2 v+1) \int_{\Omega} \gamma(x) c(x) d x+2 \delta v\left(V^{*} \circ z\right)(t)+\left(V^{*} \circ z\right)^{\prime}(t) \\
& -\int_{\Omega}\left(\delta z_{1}(t)+z_{2}(t)\right)(A \nabla \gamma) \cdot \nabla z_{1}(t) d x .
\end{aligned}
$$

De fato, com $F\left(x, z_{1}(t)(x)\right) \leq c(x)$ para quase todo $x \in \Omega$ e todo $t \in I$, temos

$$
\begin{aligned}
S^{*} & =\delta(\bar{\mu}-2 v) \int_{\Omega} \gamma(x) F\left(x, z_{1}(t)(x)\right) d x+\delta \int_{\Omega} \gamma(x) c(x) d x \\
& +2 \delta v\left(V^{*} \circ z\right)(t)+\left(V^{*} \circ z\right)^{\prime}(t)-\int_{\Omega}\left(\delta z_{1}(t)+z_{2}(t)\right)(A \nabla \gamma) \cdot \nabla z_{1}(t) d x \\
& \leq \delta(\bar{\mu}-2 v+1) \int_{\Omega} \gamma(x) c(x) d x \\
& +2 \delta v\left(V^{*} \circ z\right)(t)+\left(V^{*} \circ z\right)^{\prime}(t)-\int_{\Omega}\left(\delta z_{1}(t)+z_{2}(t)\right)(A \nabla \gamma) \cdot \nabla z_{1}(t) d x
\end{aligned}
$$

o que demonstra (4.15) e finaliza a prova do lema.

Observação 4.11. Recordemos que para $r, s \in \mathbb{R}$ temos que $|r|^{2}=|(r+s)+(-s)|^{2} \leq 2(\mid r+$ $\left.\left.s\right|^{2}+|s|^{2}\right)$ e, portanto, $|r+s|^{2} \geq(1 / 2)|r|^{2}-|s|^{2}$. Este fato será utilizado na próxima demonstração.

Demonstração do Teorema 4.9 Seja $\tau_{0} \in[0, \infty)$ arbitrário e seja $z(\cdot)=\left(z_{1}(\cdot), z_{2}(\cdot)\right)$ uma solução arbitrária de $\pi_{f}$ em $I=\left[0, \tau_{0}\right]$, com $|z(0)|_{Z} \leq R$. Considere $\bar{\gamma}=\gamma \equiv 1$ da Proposição 4.2. Segue que $s_{\bar{\gamma}}(z(t)) \equiv 0$ para todo $t \in I$ (a definição da função $s_{\bar{\gamma}}$ é dada no Lema 4.10). Segue do Lema 4.10 que

$$
\eta^{\prime}(t)+2 \delta v \eta(t) \leq \bar{c}, \text { para todo } t \in I,
$$

onde $\bar{c}=\delta(\bar{\mu}-2 v+1) \int_{\Omega} c(x) d x$. Derivando a função $t \mapsto \eta(t) e^{2 \delta v t}, t \in I$, e usando a desi- 
gualdade 4.16 obtemos

$$
\frac{d}{d t} \eta(t) e^{2 \delta v t} d t=\eta^{\prime}(t) e^{2 \delta v t}+2 \delta v \eta(t) e^{2 \delta v t}=\left(\eta^{\prime}(t)+2 \delta v \eta(t)\right) e^{2 \delta v t} \leq \bar{c} e^{2 \delta v t}, t \in I
$$

Logo,

$$
\eta(t) e^{2 \delta v t}-\eta(0) \leq \frac{\bar{c}}{2 \delta v}\left[e^{2 \delta v t}-1\right], t \in I
$$

ou seja,

$$
\eta(t) \leq \frac{\bar{c}}{2 \delta v}\left[1-e^{-2 \delta v t}\right]+\eta(0) e^{-2 \delta v t}, t \in I .
$$

Seja $C_{2} \in(0, \infty)$ uma constante tal que

$$
|u|_{L^{\bar{\rho}+2}(\Omega)} \leq C_{2}|u|_{H_{0}^{1}(\Omega)}, \text { para todo } u \in H_{0}^{1}(\Omega)
$$

Como os operadores de $H_{0}^{1}(\Omega)$ em $L^{2}(\Omega)$ induzidos por $u \mapsto|\beta|^{1 / 2} u$, respectivamente $u \mapsto$ $|a|^{1 / 2} u$, são limitados, temos que existem constantes $L_{\beta} \in(0, \infty)$, respectivamente $L_{a} \in(0, \infty)$ tais que

$$
\|\left.\left.\beta\right|^{1 / 2} u\right|_{L^{2}(\Omega)} \leq L_{\beta}|u|_{H_{0}^{1}(\Omega)}, \text { para todo } u \in H_{0}^{1}(\Omega)
$$

e

$$
\left.\left.|| a\right|^{1 / 2} u\right|_{L^{2}(\Omega)} \leq L_{a}|u|_{H_{0}^{1}(\Omega)}, \text { para todo } u \in H_{0}^{1}(\Omega) .
$$

Como $|z(0)|_{z} \leq R$, a Proposição 3.8 implica que

$$
\begin{aligned}
|\eta(0)| & =\left|V(z(0))-V^{*}(z(0))\right| \leq|V(z(0))|+\left|V^{*}(z(0))\right| \\
& \leq \frac{1}{2}\left(2 \delta^{2} \varepsilon R^{2}+2 \varepsilon R^{2}+a_{1} R^{2}+\left(L_{\beta}^{2}+\delta^{2} \varepsilon\right) R^{2}\right) \\
& +\bar{C}\left(L_{a}^{2} R^{2} / 2+\left(C_{2}\right)^{\bar{\rho}+2} R^{\bar{\rho}+2} /(\bar{\rho}+2)\right)+R \bar{\tau}=: \bar{M} .
\end{aligned}
$$

As definições das funções $V$ e $V^{*}$ e as hipóteses sobre a função $F$ implicam que para todo $t \in I$,

$$
\begin{aligned}
\frac{1}{2} \int_{\Omega}\left(\varepsilon \mid \delta z_{1}(t)(x)\right. & \left.+\left.z_{2}(t)(x)\right|^{2}+\left(A(x) \nabla z_{1}(t)(x)\right) \cdot \nabla z_{1}(t)(x)\right) d x \\
& +\frac{1}{2} \int_{\Omega}\left(\beta(x)-\delta \alpha(x)+\delta^{2} \varepsilon\right)\left|z_{1}(t)(x)\right|^{2} d x \\
& \leq \frac{\bar{c}}{2 \delta v}\left[1-e^{-2 \delta v t}\right]+\bar{M} e^{-2 \delta v t}+V^{*}(z(t)) \\
& \leq \frac{\bar{c}}{2 \delta v}\left[1-e^{-2 \delta v t}\right]+\bar{M} e^{-2 \delta v t}+\int_{\Omega} c(x) d x
\end{aligned}
$$

onde $z(t)=\left(z_{1}(t), z_{2}(t)\right) \in Z$ e $t \in I$. Utilizando em (4.18) a desiguldade da Observação 4.11 
com $r=z_{2}(t)(x)$ e $s=\delta z_{1}(t)(x)$, obtemos

$$
\begin{aligned}
\frac{1}{2} \int_{\Omega}\left((\varepsilon / 2)\left|z_{2}(t)(x)\right|^{2}\right. & \left.+\left(A(x) \nabla z_{1}(t)(x)\right) \cdot \nabla z_{1}(t)(x)+(\beta(x)-\delta \alpha(x))\left|z_{1}(t)(x)\right|^{2}\right) d x \\
& \leq \frac{\bar{c}}{2 \delta v}\left[1-e^{-2 \delta v t}\right]+\bar{M} e^{-2 \delta v t}+\int_{\Omega} c(x) d x
\end{aligned}
$$

Defina

$$
c^{\prime}=2\left[\frac{\bar{c}}{2 \delta v}+\int_{\Omega} c(x) d x\right] \text { e } M^{\prime}=2 \bar{M} .
$$

Obtemos facilmente a desigualde (4.11).

Suponha agora $|z(t)|_{Z} \leq R$, para todo $t \in I$. Seja $k \in \mathbb{N}$ arbitrário e defina $V_{k}=V_{\gamma_{k}}, V_{k}^{*}=V_{\gamma_{k}}^{*}$, $s_{k}(z)(x)=s_{\bar{\gamma}_{k}}(z)(x)$ e $\eta_{k}(t)=\eta_{\gamma_{k}}(t)$, onde $\bar{\gamma}_{k}=\bar{\vartheta}_{k}$ e $\gamma_{k}=\vartheta_{k}$. Como

$$
\nabla \vartheta_{k}(x)=\frac{2}{k^{2}} \vartheta^{\prime}\left(\frac{|x|^{2}}{k^{2}}\right) x \text { e } \nabla \bar{\vartheta}_{k}(x)=\frac{2}{k^{2}} \bar{\vartheta}^{\prime}\left(\frac{|x|^{2}}{k^{2}}\right) x, x \in \Omega,
$$

temos que

$$
\sup _{x \in \Omega}\left|\nabla \vartheta_{k}(x)\right| \leq C_{\vartheta} / k \text { e } \sup _{x \in \Omega}\left|\nabla \bar{\vartheta}_{k}(x)\right| \leq C_{\bar{\vartheta}} / k
$$

onde $C_{\vartheta}=2 \sqrt{2} \sup _{y \in \mathbb{R}}\left|\vartheta^{\prime}(y)\right|$ e $C_{\bar{\vartheta}}=2 \sqrt{2} \sup _{y \in \mathbb{R}}\left|\bar{\vartheta}^{\prime}(y)\right|$. Afirmamos que

$$
-\int_{\Omega}\left(\delta z_{1}+z_{2}\right)\left(A \nabla \vartheta_{k}\right) \cdot \nabla z_{1} d x \leq N^{2} a_{1}\left(C_{\vartheta} / k\right)(\delta R+R) R
$$

e

$$
-\delta(1-v) \int_{\Omega} s_{k}(z(t))(x) d x \leq a_{1} \delta(1-v)\left(2 N^{2} C_{\bar{\vartheta}} / k+C_{\bar{\vartheta}}^{2} / k^{2}\right) R^{2} .
$$

De fato, notemos que

$$
-\int_{\Omega}\left(\delta z_{1}+z_{2}\right)\left(A \nabla \vartheta_{k}\right) \cdot \nabla z_{1} d x \leq \int_{\Omega}\left|\left(\delta z_{1}+z_{2}\right)\left(A \nabla \vartheta_{k}\right) \cdot \nabla z_{1}\right| d x
$$

Segue do Lema 2.6 e da primeira desigualdade em 4.20) que

$$
\begin{aligned}
& \left|\left(\delta z_{1}+z_{2}\right)\left(A \nabla \vartheta_{k}\right) \cdot \nabla z_{1}\right|=\left|\left(\delta z_{1}+z_{2}\right) \sum_{i=1}^{N}\left(\sum_{j=1}^{N} a_{i j} \frac{\partial \vartheta_{k}}{\partial x_{j}}\right) \frac{\partial z_{1}}{\partial x_{i}}\right| \\
& \quad \leq\left|\delta z_{1}+z_{2}\right| \sum_{i=1}^{N}\left(\sum_{j=1}^{N} a_{i j}\left|\frac{\partial \vartheta_{k}}{\partial x_{j}}\right|\right)\left|\frac{\partial z_{1}}{\partial x_{i}}\right| \leq a_{1}\left|\delta z_{1}+z_{2}\right| \sum_{i=1}^{N}\left(\sum_{j=1}^{N}\left|\frac{\partial \vartheta_{k}}{\partial x_{j}}\right|\right)\left|\frac{\partial z_{1}}{\partial x_{i}}\right| \\
& \quad \leq N a_{1} \frac{C_{\bar{\vartheta}}}{k}\left|\delta z_{1}+z_{2}\right| \sum_{i=1}^{N}\left|\frac{\partial z_{1}}{\partial x_{i}}\right| .
\end{aligned}
$$


Logo,

$$
\begin{aligned}
-\int_{\Omega}\left(\delta z_{1}\right. & \left.+z_{2}\right)\left(A \nabla \vartheta_{k}\right) \cdot \nabla z_{1} d x \leq N a_{1} \frac{C_{\bar{\vartheta}}}{k} \sum_{i=1}^{N} \int_{\Omega}\left|\delta z_{1}+z_{2}\right|\left|\frac{\partial z_{1}}{\partial x_{i}}\right| d x \\
& \leq N a_{1} \frac{C_{\bar{\vartheta}}}{k}\left|\delta z_{1}+z_{2}\right|_{L^{2}(\Omega)} \sum_{i=1}^{N}\left|\frac{\partial z_{1}}{\partial x_{i}}\right|_{L^{2}(\Omega)} \leq N^{2} a_{1} \frac{C_{\bar{\vartheta}}}{k}\left|\delta z_{1}+z_{2}\right|_{L^{2}(\Omega)}\left|z_{1}\right|_{H^{1}(\Omega)} \\
& \leq N^{2} a_{1} \frac{C_{\bar{\vartheta}}}{k}(\delta R+R) R
\end{aligned}
$$

o que mostra a desigualdade 4.21). Para demonstrar a desigualdade 4.22 recordemos que

$$
s_{k}(z)(x)=-2 \bar{\vartheta}_{k}(x) z_{1}(x)\left(A(x) \nabla \bar{\vartheta}_{k}(x)\right) \cdot \nabla z_{1}(x)-\left|z_{1}(x)\right|^{2}\left(A(x) \nabla \bar{\vartheta}_{k}(x)\right) \cdot \nabla \bar{\vartheta}_{k}(x), x \in \Omega .
$$

Portanto

$$
\begin{aligned}
-\delta(1-v) \int_{\Omega} s_{k}(z(t))(x) d x= & -2 \delta(1-v) \int_{\Omega} \bar{\vartheta}_{k}(x) z_{1}(t)(x)\left(A(x) \nabla \bar{\vartheta}_{k}(x)\right) \cdot \nabla z_{1}(t)(x) d x \\
& -\delta(1-v) \int_{\Omega}\left|z_{1}(t)(x)\right|^{2}\left(A(x) \nabla \bar{\vartheta}_{k}(x)\right) \cdot \nabla \bar{\vartheta}_{k}(x) d x .
\end{aligned}
$$

Para obter a desigualdade 4.22, na primeira integral usamos um argumento similar ao utilizado para demonstrar a desigualdade (4.21) e na segunda integral utilizamos a desigualdade da Hipótese 2.5(a).

Defina agora

$$
\xi_{k}=\delta(\mu-2 v+1) \int_{\Omega_{k}}|c(x)| d x+N^{2} a_{1}\left(C_{\vartheta} / k\right)(\delta R+R) R+a_{1} \delta(1-v)\left(2 N^{2} C_{\bar{\vartheta}} / k+C_{\bar{\vartheta}}^{2} / k^{2}\right) R^{2},
$$

onde $\Omega_{k}=\{x \in \Omega|| x \mid \geq k\}$. Notemos que $\xi_{k} \rightarrow 0$ quando $k \rightarrow \infty$. Segue do Lema 4.10 que para cada $k \in \mathbb{N}$

$$
\eta_{k}^{\prime}+2 \delta v \eta_{k}(t) \leq \xi_{k}
$$

Derivando a função $t \mapsto \eta_{k}(t) e^{2 \delta v t}$ e utilizando um argumento similar ao usado anteriormente, segue da desigualdade 4.23 que

$$
\eta_{k}(t) \leq \frac{1}{2 \delta v} \xi_{k}\left[1-e^{-2 \delta v t}\right]+\eta_{k}(0) e^{-2 \delta v t}, \text { para } t \in I, k \in \mathbb{N}
$$

Como em 4.17), mostramos que $\left|\eta_{k}(0)\right| \leq \bar{M}$. É simples ver que as condições impostas sobre $\vartheta$ implicam que

$$
V_{k}^{*}(z(t)) \leq \int_{\Omega} \vartheta_{k}(x) c(x) d x \leq \int_{\Omega_{k}} c(x) d x=: \zeta_{k}, \text { para } t \in I, k \in \mathbb{N}
$$


Além disso, $\zeta_{k} \rightarrow 0$ quando $k \rightarrow \infty$ e para cada $t \in I$ e $k \in \mathbb{N}$,

$$
\begin{aligned}
\frac{1}{2} \int_{\Omega} \vartheta_{k}(x)\left(\varepsilon\left|\delta z_{1}(t)(x)+z_{2}(t)(x)\right|^{2}\right. & +\left(A(x) \nabla z_{1}(t)(x)\right) \cdot \nabla z_{1}(t)(x) d x \\
& \left.+\frac{1}{2} \int_{\Omega}\left(\beta(x)-\delta \alpha(x)+\delta^{2} \varepsilon\right)\left|z_{1}(t)(x)\right|^{2}\right) d x \\
& \leq \frac{1}{2 \delta v} \xi_{k}+\bar{M} e^{-2 \delta v t}+\zeta_{k} .
\end{aligned}
$$

Utilizando novamente a Observação $4.11 \operatorname{com} r=z_{2}(t)(x)$ e $s=\delta z_{1}(t)(x)$, a desiguldade acima implica que, para $t \in I$ e $k \in \mathbb{N}$,

$$
\begin{aligned}
\frac{1}{2} \int_{\Omega} \vartheta_{k}(x)\left((\varepsilon / 2)\left|z_{2}(t)(x)\right|^{2}\right. & +\left(A(x) \nabla z_{1}(t)(x)\right) \cdot \nabla z_{1}(t)(x) d x \\
& \left.+\frac{1}{2} \int_{\Omega}(\beta(x)-\delta \alpha(x))\left|z_{1}(t)(x)\right|^{2}\right) d x \\
& \leq \frac{1}{2 \delta v} \xi_{k}+\bar{M} e^{-2 \delta v t}+\zeta_{k}
\end{aligned}
$$

Defina

$$
M^{\prime}=2 \bar{M} \text { e } c_{k}=2\left((1 /(2 \delta v)) \xi_{k}+\zeta_{k}\right), k \in \mathbb{N} .
$$

Segue que a desigualdade (4.12) é válida, com $c_{k} \rightarrow 0$ quando $k \rightarrow \infty$. A demonstração do teorema está concluída.

Teorema 4.12. Assuma a Hipótese 3.9 e Hipótese 4.3. O semifluxo $\pi_{f}$ é um semifluxo global. Além disso, existe uma constante $C_{\pi_{f}} \in[0, \infty)$ com a propriedade: para todo $z_{0}$ existe um $t_{z_{0}} \in$ $[0, \infty)$ tal que $\left|z_{0} \pi_{f} t\right|_{Z} \leq C_{\pi_{f}}$ para todo $t \in\left[t_{z_{0}}, \infty\right)$. Finalmente, todo subconjunto limitado de $Z$ é u-limitado (relativamente a $\pi_{f}$ ).

Demonstração. Afirmamos que para todo $z_{0} \in Z$ existe uma constante $C_{z_{0}} \in[0, \infty)$ tal que $\left|z_{0} \pi_{f} t\right|_{Z} \leq C_{z_{0}}$ para $t \in\left[0, \omega_{z_{0}}\right)$. De fato, dado $z_{0} \in Z$ temos que $\left|z_{0}\right|_{Z} \leq R$ para algum $R \geq 0$. Seja $\left(z_{1}(t), z_{2}(t)\right)=z_{0} \pi_{f} t$ para $t \in\left[0, \omega_{z_{0}}\right)$. Consideremos $\tau \in\left[0, \omega_{z_{0}}\right)$ e seja $t \in[0, \tau]$. Segue da desigualdade 4.11) do Teorema 4.9 que

$$
\begin{aligned}
c^{\prime}+M^{\prime} e^{-2 \delta v t} & \geq \int_{\Omega}\left((\varepsilon / 2)\left|z_{2}(t)(x)\right|^{2}+\left(A(x) \nabla z_{1}(t)(x)\right) \cdot \nabla z_{1}(t)\right) d x \\
& +\int_{\Omega}(\beta(x)-\delta \alpha(x))\left|z_{1}(t)(x)\right|^{2} d x \\
& \left.=(\varepsilon / 2)\left|z_{2}(t)(x)\right|_{L^{2}(\Omega)}^{2}+\left\langle A(x) \nabla z_{1}(t)(x)\right), \nabla z_{1}(t)\right\rangle \\
& +\left\langle\beta(x) z_{1}(t)(x), z_{1}(t)(x)\right\rangle-\delta\left\langle\alpha(x) z_{1}(t)(x), z_{1}(t)(x)\right\rangle .
\end{aligned}
$$

Aqui $c^{\prime}$ e $M^{\prime}$ são como em (4.19). Notemos que estas constantes não dependem do ponto $z_{0} \mathrm{em}$ 
Z. Como $\alpha_{0} \leq \alpha(x) \leq \alpha_{1}$, uma aplicação do Lema $2.10 \operatorname{com} \kappa=\delta \alpha_{1}$ implica que para $t \in[0, \tau]$

$$
\begin{aligned}
(\varepsilon / 2)\left|z_{2}(t)(x)\right|_{L^{2}(\Omega)}^{2} & \left.+\left\langle A(x) \nabla z_{1}(t)(x)\right), \nabla z_{1}(t)\right\rangle+\left\langle\beta(x) z_{1}(t)(x), z_{1}(t)(x)\right\rangle \\
& -\delta\left\langle\alpha(x) z_{1}(t)(x), z_{1}(t)(x)\right\rangle \\
& \left.\geq(\varepsilon / 2)\left|z_{2}(t)(x)\right|_{L^{2}(\Omega)}^{2}+\left\langle A(x) \nabla z_{1}(t)(x)\right), \nabla z_{1}(t)\right\rangle \\
& +\left\langle\beta(x) z_{1}(t)(x), z_{1}(t)(x)\right\rangle-\delta \alpha_{1}\left\langle z_{1}(t)(x), z_{1}(t)(x)\right\rangle \\
& \geq(\varepsilon / 2)\left|z_{2}(t)(x)\right|_{L^{2}(\Omega)}^{2}+c\left|z_{1}(t)(x)\right|_{H_{0}^{1}(\Omega)}^{2} \geq \min ((\varepsilon / 2), c, 1)\left|z_{0} \pi_{f} t\right|_{Z}^{2},
\end{aligned}
$$

onde a constante $c$ é como no Lema 2.10. Portanto, para todo $t \in[0, \tau]$

$$
\left|z_{0} \pi_{f} t\right|_{Z}^{2} \leq[\min ((\varepsilon / 2), c, 1)]^{-1}\left(c^{\prime}+M^{\prime} e^{-2 \delta v t}\right) \leq[\min ((\varepsilon / 2), c, 1)]^{-1}\left(c^{\prime}+M^{\prime}\right) .
$$

Como $\tau \in\left[0, \omega_{z_{0}}\right)$ é arbitrário temos que

$$
\left|z_{0} \pi_{f} t\right|_{Z}^{2} \leq[\min ((\varepsilon / 2), c, 1)]^{-1}\left(c^{\prime}+M^{\prime}\right), \text { para todo } t \in\left[0, \omega_{z_{0}}\right) .
$$

Defina $C_{z_{0}}=[\min ((\varepsilon / 2), c, 1)]^{-1}\left(c^{\prime}+M^{\prime}\right)$ e afirmativa está demonstrada. A Proposição 3.1 implica que $\pi_{f}$ não explode em subconjuntos limitados. Logo, $\omega_{z_{0}}=\infty$ para todo $z_{0} \in Z$, ou seja, $\pi_{f}$ é um semifluxo global. Além disso, a relação (4.26) também implica que todo conjunto limitado de $Z$ é u-limitado.

Para concluir a prova do teorema, seja $z_{0} \in Z$. Segue da desigualdade (4.25) que

$$
\left|z_{0} \pi_{f} t\right|_{Z}^{2} \leq[\min ((\varepsilon / 2), c, 1)]^{-1}\left(c^{\prime}+M^{\prime} e^{-2 \delta v t}\right), \text { para todo } t \in[0, \infty) .
$$

Defina $C_{\pi_{f}}:=[\min ((\varepsilon / 2), c, 1)]^{-1}\left(c^{\prime}+\left(M^{\prime} / 2\right)\right)$. Como $e^{-2 \delta v t} \rightarrow 0$ quando $t \rightarrow \infty$, segue que existe um $T>0$ tal que $e^{-2 \delta v t} \leq 1 / 2$ para todo $t \geq T$. Logo

$$
\left|z_{0} \pi_{f} t\right|_{Z}^{2} \leq[\min ((\varepsilon / 2), c, 1)]^{-1}\left(c^{\prime}+M^{\prime} e^{-2 \delta v t}\right) \leq[\min ((\varepsilon / 2), c, 1)]^{-1}\left(c^{\prime}+\left(M^{\prime} / 2\right)\right),
$$

para todo $t>T$. Defina $t_{z_{0}}=T$.

\subsection{Compacidade assintótica e existência de atrator global}

Nesta seção iremos demonstrar o resultado de existência de atrator global para o semifluxo $\pi_{f}$. Em vista da Proposição 1.60 e do Teorema 4.12 resta mostrar que o semifluxo $\pi_{f}$ é assintoticamente compacto. Para demonstrar esta propriedade teremos que estudar as possibilidades 
para o valor de $\bar{\rho} \in[0, \infty)$. A primeira restrição foi imposta na Hipótese 4.3 (b). Iremos considerar o caso em que $\bar{\rho}$ é subcrítico e o caso em que $\bar{\rho}$ é crítico.

Iniciaremos com o seguinte resultado.

Proposição 4.13. Sejam a $\in C_{0}^{1}\left(\mathbb{R}^{N}\right)$ e $r \in[2, \infty)$ arbitrários. Se $N \geq 3$, assuma também que $r<2^{*}$. Então para todo $u \in H_{0}^{1}(\Omega)$, temos que $\left.a\right|_{\Omega} \cdot u \in L^{r}(\Omega)$. Além disso, a aplicação $h: H_{0}^{1}(\Omega) \rightarrow L^{r}(\Omega),\left.u \mapsto a\right|_{\Omega} \cdot u, u \in H_{0}^{1}(\Omega)$, é linear e compacta.

Demonstração. Como $a \in C_{0}^{1}\left(\mathbb{R}^{N}\right)$, existe uma bola aberta $U$ em $\mathbb{R}^{N}$ tal que supp $a \subset U$. Notemos que se $v \in H_{0}^{1}(\Omega)$, então $\widetilde{v} \in H_{0}^{1}\left(\mathbb{R}^{N}\right)$. O Lema 2.20, com $\Omega=\mathbb{R}^{N}$, implica que $\left.(a v)\right|_{U} \in H_{0}^{1}(U)$ para todo $v \in H_{0}^{1}\left(\mathbb{R}^{N}\right)$. Portanto podemos definir as aplicações

$$
\begin{aligned}
& h_{1}: H_{0}^{1}(\Omega) \rightarrow H_{0}^{1}\left(\mathbb{R}^{N}\right), v \mapsto \widetilde{v}, v \in H_{0}^{1}(\Omega), \\
& h_{2}: H_{0}^{1}\left(\mathbb{R}^{N}\right) \rightarrow H_{0}^{1}(U),\left.v \mapsto(a v)\right|_{U}, v \in H_{0}^{1}\left(\mathbb{R}^{N}\right) .
\end{aligned}
$$

Além disso, a inclusão $H_{0}^{1}(U) \subset L^{r}(U)$ induz a aplicação

$$
h_{3}: H_{0}^{1}(U) \rightarrow L^{r}(U), v \mapsto v, v \in H_{0}^{1}(U)
$$

É fácil ver que se $v \in L^{r}(U)$, então $\widetilde{v} \in L^{r}\left(\mathbb{R}^{N}\right)$ e que $\left.v\right|_{\Omega} \in L^{r}(\Omega)$ para todo $v \in L^{r}\left(\mathbb{R}^{N}\right)$. Logo, também podemos definir as aplicações

$$
\begin{aligned}
& h_{4}: L^{r}(U) \rightarrow L^{r}\left(\mathbb{R}^{N}\right), v \mapsto \widetilde{v}, v \in L^{r}(U), \\
& h_{5}: L^{r}\left(\mathbb{R}^{N}\right) \rightarrow L^{r}(\Omega),\left.v \mapsto v\right|_{\Omega}, v \in L^{r}\left(\mathbb{R}^{N}\right) .
\end{aligned}
$$

Portanto, se $u \in H_{0}^{1}(\Omega)$, temos que $\left.a\right|_{\Omega} \cdot u=\left(h_{5} \circ h_{4} \circ h_{3} \circ h_{2} \circ h_{1}\right)(u) \in L^{r}(\Omega)$. Logo

$$
h=h_{5} \circ h_{4} \circ h_{3} \circ h_{2} \circ h_{1} .
$$

É fácil ver que as aplicações $h_{i}, i \in[1, \ldots, 5]$, são todas lineares. Portanto, $h$ também é uma aplicação linear. Além disso, claramente, $h_{1}, h_{4}$ e $h_{5}$ são limitadas. Novamente uma aplicação do Lema $2.20\left(\right.$ com $\Omega=\mathbb{R}^{N}$ ) implica que $h_{2}$ é limitada. Segue do Teorema 1.11 que $h_{3}$ é uma aplicação compacta. A igualdade (4.27) implica que $h$ é uma aplicação compacta.

As seguintes hipóteses implicaram a compacidade assintótica do semifluxo $\pi_{f}$.

Hipótese 4.14. $\bar{\rho}$ é subcrítico e $\widetilde{a} \in L_{\mathrm{loc}}^{r}\left(\mathbb{R}^{N}\right)$ para algum $r \in \mathbb{R}$, com $r>\max (N, 2)$. 
Hipótese 4.15. $\bar{\rho}$ é crítico, a $\in L^{r}(\Omega)+L^{\infty}(\Omega)$ para algum $r \in[N, \infty)$ e existe uma constante $C_{1} \in[0, \infty)$ tal que

$$
|\alpha z|_{H^{-1}(\Omega)} \leq C_{1}|z|_{H^{-1}(\Omega)}, \text { para todo } z \in L^{2}(\Omega)
$$

Lema 4.16. Seja $\widetilde{N}$ um conjunto u-limitado em $Z=H_{0}^{1}(\Omega) \times L^{2}(\Omega)$ relativamente a $\pi_{f}$ arbitrário, sejam $\left(z_{n}\right)_{n}$ uma sequência em $\widetilde{N}$ e $\left(t_{n}\right)_{n}$ uma sequência em $[0, \infty)$ com $t_{n} \rightarrow \infty$ quando $n \rightarrow \infty$.

(a) Suponha a Hipótese 4.14 A sequência $\left(z_{n} \pi_{f} t_{n}\right)_{n}$ possui uma subsequência que converge em Z;

(b) Suponha a Hipótese 4.15 A sequência $\left(z_{n} \pi_{f} t_{n}\right)_{n}$ possui uma subsequência que converge em $Y=L^{2}(\Omega) \times H^{-1}(\Omega)$.

Demonstração. Como $\widetilde{N}$ é um conjunto u-limitado relativamente ao semifluxo $\pi_{f}$, existem um $t_{\widetilde{N}} \in[0, \infty)$ e um $R \in[0, \infty)$ tais que

$$
\left|z \pi_{f} t\right|_{Z} \leq R, \text { para todo } z \in \widetilde{N} \text { e para todo } t \in\left[t_{\widetilde{N}}, \infty\right)
$$

Como $t_{n} \rightarrow \infty$ quando $n \rightarrow \infty$, podemos assumir que $t_{n} \geq t_{\widetilde{N}}$ para todo $n \in \mathbb{N}$. Para cada $n \in \mathbb{N}$, defina

$$
w_{n}=z_{n} \pi_{f} t_{\widetilde{N}} \text { e } \tau_{n}=t_{n}-t_{\widetilde{N}}
$$

Notemos que se $t \in\left[0, \tau_{n}\right], n \in \mathbb{N}$, então, $t_{\widetilde{N}} \leq t+t_{\widetilde{N}} \leq t_{n}$, para todo $n \in \mathbb{N}$ e

$$
\left|w_{n} \pi_{f} t\right|=\left|\left(z_{n} \pi_{f} t_{\widetilde{N}}\right) \pi_{f} t\right|=\left|z_{n} \pi_{f}\left(t_{\widetilde{N}}+t\right)\right| \leq R, \text { para todo } n \in \mathbb{N} \text {. }
$$

Para $n \in \mathbb{N}$ e $t \in\left[0, \tau_{n}\right]$, denote $w_{n} \pi_{f} t$ pelo par $\left(u_{n}(t), v_{n}(t)\right)$. Fixe $\tau_{0} \in(0, \infty)$. Como $\tau_{n} \rightarrow \infty$ quando $n \rightarrow \infty$, existe um $n_{0}\left(\tau_{0}\right) \in \mathbb{N}$ tal que $\tau_{n} \geq 2 \tau_{0}$ para todo $n \in \mathbb{N}$ com $n \geq n_{0}\left(\tau_{0}\right)$. Para $k$, $n \in \mathbb{N} \operatorname{com} n \geq n_{0}\left(\tau_{0}\right)$ temos

$$
\begin{aligned}
w_{n} \pi_{f} \tau_{n} & =T\left(\tau_{0}\right) w_{n} \pi_{f}\left(\tau_{n}-\tau_{0}\right) \\
& +\int_{0}^{\tau_{0}} T\left(\tau_{0}-s\right)\left(0,(1 / \varepsilon)\left(\widehat{f}\left(u_{n}\left(\tau_{n}-\tau_{0}+s\right)\right)-\widehat{f}\left(\left(1-\bar{\vartheta}_{k}\right) u_{n}\left(\tau_{n}-\tau_{0}+s\right)\right)\right) d s\right. \\
& +\int_{0}^{\tau_{0}} T\left(\tau_{0}-s\right)\left(0,(1 / \varepsilon) \widehat{f}\left(\left(1-\bar{\vartheta}_{k}\right) u_{n}\left(\tau_{n}-\tau_{0}+s\right)\right)\right) d s
\end{aligned}
$$

Temos que

$$
\left|T\left(\tau_{0}\right) w_{n} \pi_{f}\left(\tau_{n}-\tau_{0}\right)\right|_{Z} \leq M e^{-\mu \tau_{0}} R, \text { para todo } n \geq n_{0}\left(\tau_{0}\right)
$$


onde $M$ e $\mu$ são as constantes positivas da Proposição 2.17. Como $\sup _{k \in \mathbb{N}}\left|\bar{\vartheta}_{k}\right|_{W^{1, \infty}\left(\mathbb{R}^{N}\right)}<\infty$, segue do Lema 2.20 que

$$
\bar{M}=\sup _{k, n \in \mathbb{N}} \sup _{t \in\left[0, \tau_{n}\right]}\left(\left|u_{n}(t)\right|_{H_{0}^{1}(\Omega)}+\left|\left(1-\bar{\vartheta}_{k}\right) u_{n}(t)\right|_{H_{0}^{1}(\Omega)}\right)<\infty
$$

As hipóteses assumidas e a Proposição 3.10 implicam que existe uma constante $L_{\bar{M}} \in(0, \infty)$ tal que, para todo $k \in \mathbb{N}, n \in \mathbb{N}$ e $t \in\left[0, \tau_{n}\right]$, temos

$$
\left|\widehat{f}\left(u_{n}(t)\right)-\widehat{f}\left(\left(1-\bar{\vartheta}_{k}\right) u_{n}(t)\right)\right|_{L^{2}(\Omega)} \leq L_{\bar{M}}\left|\bar{\vartheta}_{k} u_{n}(t)\right|_{H_{0}^{1}(\Omega)}
$$

e isso implica que, para $n \geq n_{0}\left(\tau_{0}\right)$,

$$
\begin{aligned}
\mid \int_{0}^{\tau_{0}} T\left(\tau_{0}-s\right)(0,(1 / \varepsilon) & \left.\left(\widehat{f}\left(u_{n}\left(\tau_{n}-\tau_{0}+s\right)\right)-\widehat{f}\left(\left(1-\bar{\vartheta}_{k}\right) u_{n}\left(\tau_{n}-\tau_{0}+s\right)\right)\right) d s\right|_{Z} \\
\leq & \sup _{s \in\left[0, \tau_{0}\right]}\left|\bar{\vartheta}_{k} u_{n}\left(\tau_{n}-\tau_{0}+s\right)\right|_{H_{0}^{1}(\Omega)}(1 / \varepsilon) L_{\bar{M}} M \int_{0}^{\tau_{0}} e^{-\mu\left(\tau_{0}-s\right)} d s \\
\leq & \frac{M L \bar{M}}{\mu \varepsilon} \sup _{s \in\left[0, \tau_{0}\right]}\left|\bar{\vartheta}_{k} u_{n}\left(\tau_{n}-\tau_{0}+s\right)\right|_{H_{0}^{1}(\Omega)}
\end{aligned}
$$

Agora seja $\kappa=\delta \alpha_{1}$ no Lema 2.10 e defina $c>0$ como nesse lema. Logo, para $k, n \in \mathbb{N}$ e $t \in\left[0, \tau_{n}\right]$, temos

$$
\begin{aligned}
c\left|\bar{\vartheta}_{k} u_{n}(t)\right|_{H_{0}^{1}(\Omega)}^{2} & \leq\left\langle A \nabla\left(\bar{\vartheta}_{k} u_{n}(t)\right), \nabla\left(\bar{\vartheta}_{k} u_{n}(t)\right)\right\rangle+\left\langle\beta \bar{\vartheta}_{k} u_{n}(t), \bar{\vartheta}_{k} u_{n}(t)\right\rangle-\delta \alpha_{1}\left\langle\bar{\vartheta}_{k} u_{n}(t), \bar{\vartheta}_{k} u_{n}(t)\right\rangle \\
& \leq\left\langle A \nabla\left(\bar{\vartheta}_{k} u_{n}(t)\right), \nabla\left(\bar{\vartheta}_{k} u_{n}(t)\right)\right\rangle+\left\langle\beta \bar{\vartheta}_{k} u_{n}(t), \bar{\vartheta}_{k} u_{n}(t)\right\rangle \\
& -\delta\left\langle\alpha \bar{\vartheta}_{k} u_{n}(t), \bar{\vartheta}_{k} u_{n}(t)\right\rangle .
\end{aligned}
$$

\section{Como}

$$
\begin{aligned}
\left\langle A \nabla\left(\bar{\vartheta}_{k} u_{n}(t)\right), \nabla\left(\bar{\vartheta}_{k} u_{n}(t)\right)\right\rangle & =\bar{\vartheta}_{k}^{2}\left\langle A \nabla u_{n}(t), \nabla u_{n}(t)\right\rangle \\
& +2\left\langle\bar{\vartheta}_{k} A \nabla u_{n}(t),\left(\nabla \bar{\vartheta}_{k}\right) u_{n}(t)\right\rangle+\left\langle\left(A \nabla \bar{\vartheta}_{k}\right) u_{n}(t),\left(\nabla \bar{\vartheta}_{k}\right) u_{n}(t)\right\rangle
\end{aligned}
$$

o Teorema 4.9 e a desigualdade 4.22 implicam que

$$
\begin{aligned}
c\left|\bar{\vartheta}_{k} u_{n}(t)\right|_{H_{0}^{1}(\Omega)}^{2} & \leq \int_{\Omega} \vartheta_{k}\left(A \nabla u_{n}(t)\right) \cdot \nabla u_{n}(t)+(\beta-\delta \alpha)\left|u_{n}(t)\right|_{L^{2}(\Omega)}^{2} d x \\
& +2\left\langle\bar{\vartheta}_{k} A \nabla u_{n}(t),\left(\nabla \bar{\vartheta}_{k}\right) u_{n}(t)\right\rangle+\left\langle\left(A \nabla \bar{\vartheta}_{k}\right) u_{n}(t),\left(\nabla \bar{\vartheta}_{k}\right) u_{n}(t)\right\rangle \\
& \leq c_{k}+M^{\prime} e^{-2 \delta v t}+a_{1}\left(2 N^{2} C_{\bar{\vartheta}} / k+C \bar{\vartheta}_{\vartheta}^{2} / k^{2}\right) R^{2}
\end{aligned}
$$


Para $n \geq n_{0}\left(\tau_{0}\right)$ e $s \in\left[0, \tau_{0}\right]$, temos que $t=\tau_{n}-\tau_{0}+s \geq \tau_{0}$ e, portanto, 4.30) implica que

$$
\sup _{n \geq n_{0}\left(\tau_{0}\right)} \sup _{s \in\left[0, \tau_{0}\right]}\left|\bar{\vartheta}_{k} u_{n}\left(\tau_{n}-\tau_{0}+s\right)\right|_{H_{0}^{1}(\Omega)} \rightarrow 0 \text {, quando } k \rightarrow \infty \text { e } \tau_{0} \rightarrow \infty
$$

Seja $m$ a medida de não-compacidade de Kuratowski definida na Seção 1.3. Dado $\delta>0$, seja $\tau_{0} \geq 0$ e $k_{0} \in \mathbb{N}$ tais que

$$
e^{-\mu \tau_{0}}<\frac{\delta}{4 M R}
$$

$\mathrm{e}$

$$
\sup _{n \geq n_{0}\left(\tau_{0}\right)} \sup _{s \in\left[0, \tau_{0}\right]}\left|\bar{\vartheta}_{k_{0}} u_{n}\left(\tau_{n}-\tau_{0}+s\right)\right|_{H_{0}^{1}(\Omega)}<\frac{\mu \varepsilon \delta}{4 M L_{\bar{M}}} .
$$

Temos que $\left\{w_{n} \pi_{f} \tau_{n} \mid n \in \mathbb{N}\right\} \subset A+B+C$, onde

$$
\begin{aligned}
A & =\left\{T\left(\tau_{0}\right) w_{n} \pi_{f}\left(\tau_{n}-\tau_{0}\right) \mid n \in \mathbb{N}\right\} \\
B & =\left\{\int_{0}^{\tau_{0}} T\left(\tau_{0}-s\right)\left(0,(1 / \varepsilon)\left(\widehat{f}\left(u_{n}\left(\tau_{n}-\tau_{0}+s\right)\right)-\widehat{f}\left(\left(1-\bar{\vartheta}_{k_{0}}\right) u_{n}\left(\tau_{n}-\tau_{0}+s\right)\right)\right) d s \mid n \in \mathbb{N}\right\}\right. \\
C & =\left\{\int_{0}^{\tau_{0}} T\left(\tau_{0}-s\right)\left(0,(1 / \varepsilon) \widehat{f}\left(\left(1-\bar{\vartheta}_{k_{0}}\right) u_{n}\left(\tau_{n}-\tau_{0}+s\right)\right)\right) d s \mid n \in \mathbb{N}\right\} .
\end{aligned}
$$

Portanto, a Proposição 1.17(ii) implica que

$$
m\left(\left\{w_{n} \pi_{f} \tau_{n} \mid n \in \mathbb{N}\right\}\right) \leq m(A)+m(B)+m(C) .
$$

Notemos que $A=A_{1} \cup A_{2}$, onde

$$
A_{1}=\left\{T\left(\tau_{0}\right) w_{n} \pi_{f}\left(\tau_{n}-\tau_{0}\right) \mid 1 \leq n \leq n_{0}\left(\tau_{0}\right)\right\} \text { e } A_{2}=\left\{T\left(\tau_{0}\right) w_{n} \pi_{f}\left(\tau_{n}-\tau_{0}\right) \mid n \geq n_{0}\left(\tau_{0}\right)\right\}
$$

Como $A_{1}$ é um conjunto finito, segue que é compacto e, portanto, $m\left(A_{1}\right)=0$. Logo, $m(A)=$ $\max \left\{m\left(A_{1}\right), m\left(A_{2}\right)\right\}=m\left(A_{2}\right)$. As desigualdades (4.28) e (4.32) implicam que

$$
\left|T\left(\tau_{0}\right) w_{n} \pi_{f}\left(\tau_{n}-\tau_{0}\right)\right|_{Z} \leq M e^{-\mu \tau_{0}} R \leq M R \frac{\delta}{4 M R}=\frac{\delta}{4}, \text { para todo } n \geq n_{0}\left(\tau_{0}\right),
$$

ou seja, mostramos que $A_{2} \subset B(0, \delta / 4)$. Logo, $m\left(A_{2}\right) \leq m(B(0, \delta / 4))=\delta / 2$ e obtemos

$$
m(A) \leq \delta / 2
$$


Além disso, $B=B_{1} \cup B_{2}$, onde

$$
B_{1}=\left\{\int_{0}^{\tau_{0}} \Psi_{n}(s) d s \mid 1 \leq n \leq n_{0}\left(\tau_{0}\right)\right\} \text { e } B_{2}=\left\{\int_{0}^{\tau_{0}} \Psi_{n}(s) d s \mid n \geq n_{0}\left(\tau_{0}\right)\right\} .
$$

Aqui, $\Psi_{n}(s):=T\left(\tau_{0}-s\right)\left(0,(1 / \varepsilon)\left(\widehat{f}\left(u_{n}\left(\tau_{n}-\tau_{0}+s\right)\right)-\widehat{f}\left(\left(1-\bar{\vartheta}_{k_{0}}\right) u_{n}\left(\tau_{n}-\tau_{0}+s\right)\right)\right)\right)$, para $n \in$ $\mathbb{N}$. Novamente temos que $B_{1}$ é um conjunto finito e, portanto, $m\left(B_{1}\right)=0$. Assim, $m(B)=$ $\max \left\{m\left(B_{1}\right), m\left(B_{2}\right)\right\}=m\left(B_{2}\right)$. As desigualdades 4.29) e 4.33) implicam que

$$
\begin{aligned}
\left|\Psi_{n}(s)\right| & \leq \frac{M L_{\bar{M}}}{\mu \varepsilon} \sup _{n \geq n_{0}\left(\tau_{0}\right)} \sup _{s \in\left[0, \tau_{0}\right]}\left|\bar{\vartheta}_{k_{0}} u_{n}\left(\tau_{n}-\tau_{0}+s\right)\right|_{H_{0}^{1}(\Omega)} \\
& <\left(\frac{M L_{\bar{M}}}{\mu \varepsilon}\right)\left(\frac{\mu \varepsilon \delta}{4 M L_{\bar{M}}}\right)=\frac{\delta}{4} \text {, para todo } n \geq n_{0}\left(\tau_{0}\right) .
\end{aligned}
$$

Segue que

$$
m(B)=m\left(B_{2}\right) \leq m(B(0, \delta / 4))=\delta / 2
$$

Finalmente, notemos que $C=C_{1} \cup C_{2}$, onde

$$
\begin{aligned}
& C_{1}=\left\{\int_{0}^{\tau_{0}} T\left(\tau_{0}-s\right)\left(0,(1 / \varepsilon) \widehat{f}\left(\left(1-\bar{\vartheta}_{k}\right) u_{n}\left(t-\tau_{0}+s\right)\right)\right) d s \mid 1 \leq n \leq n_{0}\left(\tau_{0}\right)\right\}, \\
& C_{2}=\left\{\int_{0}^{\tau_{0}} T\left(\tau_{0}-s\right)\left(0,(1 / \varepsilon) \widehat{f}\left(\left(1-\bar{\vartheta}_{k}\right) u_{n}\left(t-\tau_{0}+s\right)\right)\right) d s \mid n \geq n_{0}\left(\tau_{0}\right)\right\} .
\end{aligned}
$$

Como anteriormente, $m\left(C_{1}\right)=0$. Afirmamos que

$(\triangleright) K_{0}=\left\{T\left(\tau_{0}-s\right)\left(0,(1 / \varepsilon) \widehat{f}\left(\left(1-\bar{\vartheta}_{k}\right) u_{n}\left(\tau_{n}-\tau_{0}+s\right)\right)\right) \mid n \geq n_{0}\left(\tau_{0}\right), s \in\left[0, \tau_{0}\right]\right\}$ é relativamente compacto em $Z$ (respectivamente, em $Y$ ).

Suponha que a afirmativa acima seja verdadeira. Defina $K_{1}=\overline{K_{0}}$. O Teorema de Mazur (ver Teorema 1.19 implica que $\tau_{0} \overline{\operatorname{co}_{1}}$ é um conjunto compacto, onde co $K_{1}$ é a envoltória convexa do conjunto $K_{1}$. Logo, $m\left(\tau_{0} \overline{\operatorname{co} K_{1}}\right)=0$. Recordemos que para cada $n \geq n_{0}\left(\tau_{0}\right)$ temos

$$
\int_{0}^{\tau_{0}} T\left(\tau_{0}-s\right)\left(0,(1 / \varepsilon) \widehat{f}\left(\left(1-\bar{\vartheta}_{k}\right) u_{n}\left(t-\tau_{0}+s\right)\right)\right) d s=\lim _{j \rightarrow \infty} \int_{0}^{\tau_{0}} \phi_{j}(s) d s,
$$

onde $\phi_{j}, j \in \mathbb{N}$, é uma função simples. A definição da integral de Bochner (ver [12] ou [21]) implica que $\int_{0}^{\tau_{0}} \phi_{j}(s) d s \in \tau_{0} \overline{\operatorname{co} K_{1}}$ para todo $j \in \mathbb{N}$. Logo, $C_{2} \subset \tau_{0} \overline{\operatorname{co} K_{1}}$ e $m\left(C_{2}\right)=0$. Portanto,

$$
m(C)=m\left(C_{2}\right)=0
$$


Utilizando fórmulas 4.35)-4.37) na desigualdade (4.34), obtemos

$$
m\left(\left\{w_{n} \pi_{f} \tau_{n} \mid n \in \mathbb{N}\right\}\right) \leq 2(\delta / 2)=\delta, \text { para todo } \delta>0
$$

Fazendo $\delta \rightarrow 0$ em (4.38) obtemos que $m\left(\left\{w_{n} \pi_{f} \tau_{n} \mid n \in \mathbb{N}\right\}\right)=0$, ou seja, $\left\{w_{n} \pi_{f} \tau_{n} \mid n \in \mathbb{N}\right\}$ é um conjunto relativamente compacto em $Z$ (respectivamente, em $Y$ ).

Para completar a demonstração basta mostrar a afirmativa $(\triangleright)$. Seja $\left(w_{l}\right)_{l}$ uma sequência em $K_{0}$. Segue que, para todo $l \in \mathbb{N}$, existem um $n_{l} \in \mathbb{N}$ e um $s_{l} \in\left[0, \tau_{0}\right]$ com $w_{l}=$ $T\left(\tau_{0}-s_{l}\right)\left(0,(1 / \varepsilon) \widehat{f}\left(v_{l}\right)\right)$, onde $v_{l}=\left(1-\bar{\vartheta}_{k}\right) u_{n_{l}}\left(\tau_{n_{l}}-\tau_{0}+s_{l}\right)$. Escolhendo subsequências se necessário, podemos assumir que $s_{l} \rightarrow s_{\infty}$ para algum $s_{\infty} \in\left[0, \tau_{0}\right]$. Como $\left(1-\bar{\vartheta}_{k}\right) \in C_{0}^{1}\left(\mathbb{R}^{N}\right)$, segue da Proposição 4.13 que o conjunto $\left\{\left(v_{l}\right)_{l} \mid l \in \mathbb{N}\right\}$ é compacto em $L^{S}(\Omega)$ para cada $s \in[2, \infty)$, com $s \in\left[2,2^{*}\right)$ se $N \geq 3$.

Suponha que a Hipótese 4.14 seja válida. Para $s \in\{2 r /(r-2), 2(\bar{\rho}+1)\}$, temos que $s \in$ $\left[2,2^{*}\right)$. Como $\left\{v_{l} \mid l \in \mathbb{N}\right\}$ é um conjunto limitado e $H_{0}^{1}(\Omega)$ é reflexivo, tomando subsequências se necessário, podemos assumir que existe um $v \in H_{0}^{1}(\Omega)$ tal que $v_{l} \rightarrow v$ em $H_{0}^{1}(\Omega)$. Além disso, podemos supor ainda que $v_{l} \rightarrow v$ em $L^{s}(\Omega)$, para $s \in\{2 r /(r-2), 2(\bar{\rho}+1)\}$. Notemos que para todo $x \in \Omega$ com $|x| \geq \sqrt{2} k$, então $v_{l}(x)=0$ para todo $l \in \mathbb{N}$ e, portanto, podemos assumir $v(x)=0$ nesse caso. Logo,

$$
a(x)\left(v_{l}(x)-v(x)\right)=a_{1}\left(v_{l}(x)-v(x)\right), l \in \mathbb{N}, x \in \Omega,
$$

onde $a_{1}: \mathbb{R}^{N} \rightarrow \mathbb{R}$ é definida por

$$
a_{1}(x)=\left\{\begin{array}{l}
\widetilde{a}(x), \text { se } x \in \bar{\Omega} \mathrm{e}|x| \leq \sqrt{2} k \\
0, \text { caso contrário. }
\end{array}\right.
$$

Como $\widetilde{a} \in L_{\mathrm{loc}}^{r}(\Omega)$, temos que $a_{1} \in L^{r}\left(\mathbb{R}^{N}\right)$ e segue que a aplicação de $L^{2 r /(r-2)}(\Omega)$ em $L^{2}(\Omega)$ dada por $h \mapsto a_{1} h$ está bem definida e é linear. Como, para $h \in L^{2 r /(r-2)}(\Omega)$ temos

$$
\int_{\Omega}\left|a_{1} h\right|^{2} d x=\int_{\Omega}\left|a_{1}\right|^{2}|h|^{2} d x \leq\left(\int_{\Omega}\left|a_{1}\right|^{r}\right)^{1 / r}\left(\int_{\Omega}|h|^{2 r / r-2}\right)^{r-2 / 2 r},
$$

segue que $h$ é limitada. Agora, como $v, v_{l}, v_{l}-v \in L^{S}(\Omega)$ para $s \in\{2 r /(r-2), 2(\bar{\rho}+1)\}$, a desigualdade (3.9) e igualdade 4.39) implicam que

$$
\left|\widehat{f}\left(v_{l}\right)-\widehat{f}(v)\right|_{L^{2}(\Omega)} \rightarrow 0, \text { quando } l \rightarrow \infty \text {. }
$$


Afirmamos que $\left|w_{l}-T\left(\tau_{0}-s_{\infty}\right)(0,(1 / \varepsilon) \widehat{f}(v))\right|_{Z} \rightarrow 0$ quando $l \rightarrow \infty$. De fato,

$$
\begin{aligned}
\left|w_{l}-T\left(\tau_{0}-s_{\infty}\right)(0,(1 / \varepsilon) \widehat{f}(v))\right|_{Z} & =\left|T\left(\tau_{0}-s_{l}\right)\left(0,(1 / \varepsilon) \widehat{f}\left(v_{l}\right)\right)-T\left(\tau_{0}-s_{\infty}\right)(0,(1 / \varepsilon) \widehat{f}(v))\right|_{Z} \\
& \leq\left|T\left(\tau_{0}-s_{l}\right)\left(0,(1 / \varepsilon) \widehat{f}\left(v_{l}\right)\right)-T\left(\tau_{0}-s_{l}\right)(0,(1 / \varepsilon) \widehat{f}(v))\right|_{Z} \\
& +\left|T\left(\tau_{0}-s_{l}\right)(0,(1 / \varepsilon) \widehat{f}(v))-T\left(\tau_{0}-s_{\infty}\right)(0,(1 / \varepsilon) \widehat{f}(v))\right|_{Z}
\end{aligned}
$$

Notemos que

$$
\begin{aligned}
\mid T\left(\tau_{0}-s_{l}\right)\left(0,(1 / \varepsilon) \widehat{f}\left(v_{l}\right)\right) & -\left.T\left(\tau_{0}-s_{l}\right)(0,(1 / \varepsilon) \widehat{f}(v))\right|_{Z} \\
& \left.=\mid T\left(\tau_{0}-s_{l}\right)\left(\left(0,(1 / \varepsilon) \widehat{f}\left(v_{l}\right)\right)-\widehat{f}(v)\right)\right)\left.\right|_{Z} \\
& \leq M e^{\mu\left(\tau_{0}-s_{l}\right)}\left|\left(0,(1 / \varepsilon) \widehat{f}\left(v_{l}\right)\right)-(0,(1 / \varepsilon) \widehat{f}(v))\right|_{Z} \\
& =M e^{\mu\left(\tau_{0}-s_{l}\right.}\left|\widehat{f}\left(v_{l}\right)-\widehat{f}(v)\right|_{L^{2}(\Omega)},
\end{aligned}
$$

onde $M, \mu$ são como na Proposição 2.17 e que

$T\left(\tau_{0}-s_{l}\right)(0,(1 / \varepsilon) \widehat{f}(v))-T\left(\tau_{0}-s_{\infty}\right)(0,(1 / \varepsilon) \widehat{f}(v))=\left(T\left(\tau_{0}-s_{l}\right)-T\left(\tau_{0}-s_{\infty}\right)\right)(0,(1 / \varepsilon) \widehat{f}(v))$

Portanto, a fórmula 4.40) e o Teorema 1.24(i) implicam que

$$
\left|w_{l}-T\left(\tau_{0}-s_{\infty}\right)(0,(1 / \varepsilon) \widehat{f}(v))\right|_{Z} \rightarrow 0
$$

quando $l \rightarrow \infty$. Como $w_{n} \pi_{f} \tau_{n}=\left(z_{n} \pi_{f} t_{\widetilde{N}}\right) \pi_{f} \tau_{n}=z_{n} \pi_{f} t_{n}, n \in \mathbb{N}$, segue que a sequência $\left(z_{n} \pi_{f} t_{n}\right)_{n}$ possui subsequência convergente em $Z$ e $(i)$ está demonstrada.

Agora suponha a Hipótese 4.15 seja válida. Temos que $2 \in\left[2,2^{*}\right)$ para $N \geq 3$. Tomando subsequências se necessário, podemos assumir, de maneira análoga ao feito em $(i)$, que existe um $v \in H_{0}^{1}(\Omega)$ tal que $v_{l} \rightarrow v$ em $H_{0}^{1}(\Omega)$ e $v_{l} \rightarrow v$ em $L^{s}(\Omega)$. Usando (3.13) obtemos que $\mid \widehat{f}\left(v_{l}\right)-$ $\left.\widehat{f}(v)\right|_{H^{-1}(\Omega)} \rightarrow 0$ quando $l \rightarrow \infty$. A Proposição 2.19 implica, como em $(i)$, que $\mid w_{l}-T\left(\tau_{0}-\right.$ $\left.s_{\infty}\right)\left.(0,(1 / \varepsilon) \widehat{f}(v))\right|_{Y} \rightarrow 0$ quando $l \rightarrow \infty$ e, finalmente, a sequência $\left(z_{n} \pi_{f} t_{n}\right)_{n}$ possui subsequência convergente em $Y$.

Com isso, temos o seguinte resultado de existência de atrator global para o caso subcrítico:

Teorema 4.17. Assuma a Hipótese 3.9. a Hipótese 4.3 e a Hipótese 4.14 O semifluxo $\pi_{f}$ é um semifluxo global e possui um atrator global.

Demonstração. O Teorema 4.12 implica que $\pi_{f}$ é um semifluxo global. Além disso, segue do Teorema 4.12 que todo conjunto limitado é u-limitado e que existe um conjunto limitado $B_{0}$ 
tal que para todo $z \in Z$ existe um $t_{z} \in[0, \infty)$ com $z \pi_{f} t_{z} \in B_{0}$. Segue do Lema 4.16 que $\pi_{f}$ é assintoticamente compacto. Agora, uma aplicação da Proposição 1.60 implica que o semifluxo global $\pi_{f}$ possui um atrator global.

Agora passemos à analise com o caso crítico. Temos que demonstrar que $\pi_{f}$ é assintoticamente compacto em $Z$. Começaremos com o seguinte resultado auxiliar.

Proposição 4.18. Assuma Hipótese 3.9. Hipótese 4.3 e Hipótese 4.15. Seja $C_{6} \in[0, \infty)$ uma constante arbitrária. Então existe uma constante $C_{7} \in[0, \infty)$ tal que para todo $t \in[0, \infty)$ e $z$, $w \in Z$, com $|z|_{Z} \leq C_{6} e|w|_{Z} \leq C_{6}$, temos que

$$
\left|z \pi_{f} t-w \pi_{f} t\right|_{Y} \leq C_{7} e^{C_{7} t}|z-w|_{Y}
$$

Demonstração. A Proposição 3.1 implica que

$$
z \pi_{f} t=T(t) z+\int_{0}^{t} T(t-s)\left(0, \widehat{f}\left(z_{1}(s)\right)\right) d s, \text { para todo } t \in[0, \infty) \text { e para todo } z \in Z \text {. }
$$

Assim, para obter a desigualdade (4.41) devemos encontrar estimativas para o lado direito da igualdade acima. A Hipótese 4.15 implica que as condições da Proposição 2.19 estão satisfeitas. Logo,

$$
|T(t) z|_{Y} \leq C_{2} e^{C_{3} t}|z|_{Y}, \text { para todo } t \in[0, \infty) \text { e para todo } z \in Z,
$$

onde as constantes $C_{2}$ e $C_{3}$ são como naquela proposição. Segue do Teorema 4.9 e do Lema 2.10 que existe uma constante $C_{8} \in[0, \infty)$ tal que para qualquer que seja $z \in Z$ com $|z|_{Z} \leq C_{6}$, então

$$
\left|z \pi_{f} t\right|_{Z} \leq C_{8} \text {, para todo } t \in[0, \infty)
$$

Além disso, afirmamos que existe uma constante $C_{9} \in[0, \infty)$ tal que

$$
\left|\widehat{f}\left(z_{1}\right)-\widehat{f}\left(w_{1}\right)\right|_{H^{-1}(\Omega)} \leq C_{9}\left|z_{1}-w_{1}\right|_{L^{2}(\Omega)}
$$

para todo $z=\left(z_{1}, z_{2}\right), w=\left(w_{1}, w_{2}\right) \in Z$ com $|z|_{Z} \leq C_{8}$ e $|w|_{Z} \leq C_{8}$. De fato, segue da Proposição 3.8 (ver fórmula (3.13) ) que para todo $z=\left(z_{1}, z_{2}\right), w=\left(w_{1}, w_{2}\right) \in Z$ temos que existe uma constante $C(t) \geq 0$ tal que

$$
\begin{aligned}
\left|\widehat{f}\left(z_{1}\right)-\widehat{f}\left(w_{1}\right)\right|_{H^{-1}(\Omega)} & \leq C(r)\left(\left|a_{1}\right|_{L^{r}(\Omega}+\left|a_{2}\right|_{L^{\infty}(\Omega)}\right)\left|z_{1}-w_{1}\right|_{L^{2}(\Omega)} \\
& +C(r)\left(\left|w_{1}\right|_{L^{2^{*}(\Omega)}}^{\bar{\rho}}+\left|z_{1}-w_{1}\right|_{L^{2^{*}(\Omega)}}^{\bar{\rho}}\right)\left|z_{1}-w_{1}\right|_{L^{2}(\Omega)} .
\end{aligned}
$$


O Teorema 1.10 agora implica que

$$
\begin{aligned}
\left|\widehat{f}\left(z_{1}\right)-\widehat{f}\left(w_{1}\right)\right|_{H^{-1}(\Omega)} & \leq C(r) \widetilde{K}\left|z_{1}-w_{1}\right|_{L^{2}(\Omega)} \\
& +C(r)\left(K_{2^{*}}^{\bar{\rho}}\left|w_{1}\right|_{H_{0}^{1}(\Omega)}^{\bar{\rho}}+K_{2^{*}}^{\bar{\rho}}\left|z_{1}-w_{1}\right|_{H_{0}^{1}(\Omega)}^{\bar{\rho}}\right)\left|z_{1}-w_{1}\right|_{L^{2}(\Omega)}
\end{aligned}
$$

onde $K_{2^{*}}$ é como em Teorema 1.10 e $\widetilde{K}=\left|a_{1}\right|_{L^{r}(\Omega}+\left|a_{2}\right|_{L^{\infty}(\Omega)}$. Portanto, para todo $z=$ $\left(z_{1}, z_{2}\right), w=\left(w_{1}, w_{2}\right) \in Z$ com $|z|_{Z} \leq C_{8}$ e $|w| z \leq C_{8}$ temos

$$
\begin{aligned}
\mid \widehat{f}\left(z_{1}\right) & -\left.\widehat{f}\left(w_{1}\right)\right|_{H^{-1}(\Omega)} \leq C(r) \widetilde{K}\left|z_{1}-w_{1}\right|_{L^{2}(\Omega)} \\
& +C(r)\left(K_{2^{*}}^{\bar{\rho}}\left|w_{1}\right|_{H_{0}^{1}(\Omega)}^{\bar{\rho}}+K_{2^{*}}^{\bar{\rho}} \max \left(1,2^{\bar{\rho}-1}\right)\left(\left|z_{1}\right|_{H_{0}^{1}(\Omega)}^{\bar{\rho}}+\left|w_{1}\right|_{H_{0}^{1}(\Omega)}^{\bar{\rho}}\right)\right)\left|z_{1}-w_{1}\right|_{L^{2}(\Omega)} \\
& \leq\left(C(r) \widetilde{K}+C(r)\left(K_{2^{*}}^{\bar{\rho}} C_{8}^{\bar{\rho}}+K_{2^{*}}^{\bar{\rho}} \max \left(1,2^{\bar{\rho}-1}\right)\left(C_{8}^{\bar{\rho}}+C_{8}^{\bar{\rho}}\right)\right)\right)\left|z_{1}-w_{1}\right|_{L^{2}(\Omega)} .
\end{aligned}
$$

Defina $C_{9}=C(r) \widetilde{K}+C(r)\left(K_{2^{*}}^{\bar{\rho}} C_{8}^{\bar{\rho}}+K_{2^{*}}^{\bar{\rho}} \max \left(1,2^{\bar{\rho}-1}\right)\left(C_{8}^{\bar{\rho}}+C_{8}^{\bar{\rho}}\right)\right)$. A prova da afirmativa está completa.

Sejam $z, w \in Z \operatorname{com}|z|_{Z} \leq C_{6}$ e $|w|_{Z} \leq C_{6}$. Segue das desigualdades (4.42)-4.44) que

$$
\begin{aligned}
\mid z \pi_{f} t & -\left.w \pi_{f} t\right|_{Y} \leq|T(t)(z-w)|_{Y}+\int_{0}^{t}\left|T(t-s)\left(0, \widehat{f}\left(z_{1}(s)\right)-\widehat{f}\left(w_{1}(s)\right)\right)\right|_{Y} d s \\
& \leq C_{2} e^{C_{3} t}|z-w|_{Y}+\int_{0}^{t} C_{2} e^{C_{3}(t-s)}\left|\left(0, \widehat{f}\left(z_{1}(s)\right)-\widehat{f}\left(w_{1}(s)\right)\right)\right|_{Y} d s \\
& \leq C_{2} e^{C_{3} t}|z-w|_{Y}+\int_{0}^{t} C_{2} e^{C_{3}(t-s)}\left|\widehat{f}\left(z_{1}(s)\right)-\widehat{f}\left(w_{1}(s)\right)\right|_{H^{-1}(\Omega)} d s \\
& \leq C_{2} e^{C_{3} t}|z-w|_{Y}+\int_{0}^{t} C_{2} C_{9} e^{C_{3}(t-s)}\left|z_{1}(s)-w_{1}(s)\right|_{L^{2}(\Omega)} d s \\
& \leq C_{2} e^{C_{3} t}|z-w|_{Y}+\int_{0}^{t} C_{2} C_{9} e^{C_{3}(t-s)}\left|z \pi_{f} s-w \pi_{f} s\right|_{Y} d s .
\end{aligned}
$$

Aqui usamos a notação $z \pi_{f} t=\left(z_{1}(t), z_{2}(t)\right)$ e $w \pi_{f} t=\left(w_{1}(t), w_{2}(t)\right), t \in[0, \infty)$. Agora uma aplicação da Lema de Gronwall (ver Lema 1.61) obtemos

$$
\left|z \pi_{f} t-w \pi_{f} t\right|_{Y} \leq C_{2}|z-w|_{Y} e^{\left(C_{3}+C_{2} C_{9}\right) t}, \text { para todo } t \in[0, \infty)
$$

Logo, para concluir a demonstração basta definir $C_{7}:=\max \left(C_{2}, C_{3}+C_{2} C_{9}\right)$.

Estamos prontos para mostrar que o semifluxo $\pi_{f}$ é assintoticamente compacto em $Z$ para o caso crítico. O método utilizado em [26] adapta as técnicas apresentadas em [4].

Teorema 4.19. Assuma a Hipótese 3.9 a Hipótese 4.3 e a Hipótese 4.15 Então o semifluxo $\pi_{f}$ é assintoticamente compacto em $\mathrm{Z}$. 
Para demonstrar o Teorema 4.19 vamos utilizar o seguinte lema auxiliar.

Lema 4.20. Assuma as hipóteses do Teorema 4.19 A aplicação $\Psi: Z \rightarrow Z$, definida por $\Psi(u, v)=(u, \delta u+v)$, para $(u, v) \in Z$ é um isomorfismo. Além disso, a forma bilinear

$$
\left[\left(u_{1}, v_{1}\right),\left(u_{2}, v_{2}\right)\right]:=\varepsilon\left\langle\delta u_{1}+v_{1}, \delta u_{2}+v_{2}\right\rangle+\left\langle A \nabla u_{1}, \nabla u_{2}\right\rangle+\left\langle\left(\beta-\delta \alpha+\delta^{2} \varepsilon\right) u_{1}, u_{2}\right\rangle,
$$

para $\left(u_{1}, v_{1}\right),\left(u_{2}, v_{2}\right) \in Z$ define um produto interno em $Z$ cuja norma $z \in Z \mapsto\|z\|:=\sqrt{[z, z]} \dot{e}$ equivalente à norma usual de $\mathrm{Z}$

Demonstração. É fácil mostrar que $\Psi$ é um isomorfismo e que $[\cdot, \cdot]$ é um produto interno em $Z$. Para completar a demonstração temos que mostrar que a norma $\|\cdot\|$ é equivalente à norma usual de $Z$. Seja $\left(u_{1}, v_{1}\right) \in Z$. Temos que

$$
\left[\left(u_{1}, v_{1}\right),\left(u_{1}, v_{1}\right)\right]=\varepsilon\left\langle\delta u_{1}+v_{1}, \delta u_{1}+v_{1}\right\rangle+\left\langle A \nabla u_{1}, \nabla u_{1}\right\rangle+\left\langle\left(\beta-\delta \alpha+\delta^{2} \varepsilon\right) u_{1}, u_{1}\right\rangle
$$

Notemos que

$$
\left\langle\delta u_{1}+v_{1}, \delta u_{1}+v_{1}\right\rangle=\left|\delta u_{1}+v_{1}\right|_{L^{2}(\Omega)}^{2} \leq\left|\nabla u_{1}\right|_{L^{2}(\Omega)}^{2}+\delta\left|u_{1}\right|_{L^{2}(\Omega)}^{2}+\left|v_{1}\right|_{L^{2}(\Omega)}^{2} .
$$

Segue do Lema 2.10, $\operatorname{com} \kappa=\delta \alpha_{1}$, que

$$
c\left|u_{1}\right|_{H_{0}^{1}(\Omega)}^{2} \leq\left\langle A \nabla u_{1}, \nabla u_{1}\right\rangle+\left\langle\beta u_{1}, u_{1}\right\rangle-\delta \alpha_{1}\left\langle u_{1}, u_{1}\right\rangle \leq C\left|u_{1}\right|_{H_{0}^{1}(\Omega)}^{2}
$$

onde as contantes $c$ e $C$ são como no Lema2.10, Portanto

$$
\left\langle A \nabla u_{1}, \nabla u_{1}\right\rangle+\left\langle\left(\beta-\delta \alpha+\delta^{2} \varepsilon\right) u_{1}, u_{1}\right\rangle \leq C\left|u_{1}\right|_{H_{0}^{1}(\Omega)}^{2}+\delta \alpha_{1}\left\langle u_{1}, u_{1}\right\rangle+\left\langle\left(-\delta \alpha+\delta^{2} \varepsilon\right) u_{1}, u_{1}\right\rangle
$$

Segue da Hipótese 2.16 que $\alpha_{0} \leq \alpha(x) \leq \alpha_{1}$ para quase todo $x$ em $\Omega$. Logo, $-\left\langle\delta \alpha u_{1}, u_{1}\right\rangle \leq$ $-\delta \alpha_{0}\left|u_{1}\right|_{L^{2}(\Omega)}^{2}$ e obtemos

$$
\left\langle A \nabla u_{1}, \nabla u_{1}\right\rangle+\left\langle\left(\beta-\delta \alpha+\delta^{2} \varepsilon\right) u_{1}, u_{1}\right\rangle \leq C\left|u_{1}\right|_{H_{0}^{1}(\Omega)}^{2}+\left(\delta\left(\alpha_{1}-\alpha_{0}\right)+\delta^{2} \varepsilon\right)\left|u_{1}\right|_{L^{2}(\Omega)}^{2}
$$

Notemos que $\delta\left(\alpha_{1}-\alpha_{0}\right)+\delta^{2} \varepsilon>0$. As desigualdades (4.45) e (4.46) implicam que

$$
\left[\left(u_{1}, v_{1}\right),\left(u_{1}, v_{1}\right)\right] \leq(\varepsilon+C)\left|\nabla u_{1}\right|_{L^{2}(\Omega)}^{2}+\left(\delta \varepsilon+\delta\left(\alpha_{1}-\alpha_{0}\right)+\delta^{2} \varepsilon\right)\left|u_{1}\right|_{L^{2}(\Omega)}^{2}+\varepsilon\left|v_{1}\right|_{L^{2}(\Omega)}^{2}
$$


Portanto,

$$
\left[\left(u_{1}, v_{1}\right),\left(u_{1}, v_{1}\right)\right] \leq \max \left\{\varepsilon+C, \delta \varepsilon+\delta\left(\alpha_{1}-\alpha_{0}\right)+\delta^{2} \varepsilon, \varepsilon\right\}\left|\left(u_{1}, v_{1}\right)\right|_{Z}^{2}
$$

Por outro lado, temos que

$$
\begin{gathered}
\left\langle\delta u_{1}+v_{1}, \delta u_{1}+v_{1}\right\rangle=\left|\delta u_{1}+v_{1}\right|_{L^{2}(\Omega)}^{2} \geq\left|v_{1}\right|_{L^{2}(\Omega)}^{2}-\delta^{2}\left|u_{1}\right|_{L^{2}(\Omega)}^{2}, \\
\left\langle A \nabla u_{1}, \nabla u_{1}\right\rangle+\left\langle\left(\beta-\delta \alpha+\delta^{2} \varepsilon\right) u_{1}, u_{1}\right\rangle \geq c\left|u_{1}\right|_{H_{0}^{1}(\Omega)}^{2}+\delta \alpha_{1}\left\langle u_{1}, u_{1}\right\rangle+\left\langle\left(-\delta \alpha+\delta^{2} \varepsilon\right) u_{1}, u_{1}\right\rangle .
\end{gathered}
$$

Como anteriormente temos que $-\left\langle\delta \alpha u_{1}, u_{1}\right\rangle \geq-\delta \alpha_{1}\left|u_{1}\right|_{L^{2}(\Omega)}^{2}$ e obtemos

$$
\left\langle A \nabla u_{1}, \nabla u_{1}\right\rangle+\left\langle\left(\beta-\delta \alpha+\delta^{2} \varepsilon\right) u_{1}, u_{1}\right\rangle \geq c\left|u_{1}\right|_{H_{0}^{1}(\Omega)}^{2}+\delta^{2} \varepsilon\left|u_{1}\right|_{L^{2}(\Omega)}^{2} .
$$

Segue das desigualdades 4.48) e 4.49) que

$$
\left[\left(u_{1}, v_{1}\right),\left(u_{1}, v_{1}\right)\right] \geq \varepsilon\left|v_{1}\right|_{L^{2}(\Omega)}^{2}-\delta^{2} \varepsilon\left|u_{1}\right|_{L^{2}(\Omega)}^{2}+c\left|u_{1}\right|_{H_{0}^{1}(\Omega)}^{2}+\delta^{2} \varepsilon\left|u_{1}\right|_{L^{2}(\Omega)}^{2}
$$

Portanto,

$$
\left[\left(u_{1}, v_{1}\right),\left(u_{1}, v_{1}\right)\right] \geq \varepsilon\left|v_{1}\right|_{L^{2}(\Omega)}^{2}+c\left|u_{1}\right|_{H_{0}^{1}(\Omega)}^{2} \geq \min \{\varepsilon, c\}\left|\left(u_{1}, v_{1}\right)\right|_{Z}^{2} .
$$

As desigualdades 4.47) e 4.50) implicam o lema.

Demonstração do Teorema 4.19 Seja $\widetilde{N}$ um conjunto u-limitado de $Z$. Então existe um $t_{\widetilde{N}} \in$ $[0, \infty)$ e uma constante $C_{10} \in[0, \infty)$ tais que

$$
\left|z \pi_{f} t\right| \leq C_{10} \text {, para todo } z \in \widetilde{N} \text { e } t \geq t_{\widetilde{N}}
$$

Seja $\left(z_{n}\right)_{n}$ uma sequência em $\widetilde{N}$ e $\left(t_{n}\right)_{n}$ uma sequência em $[0, \infty)$ com $t_{n} \rightarrow \infty$ quando $n \rightarrow \infty$. Devemos provar que $\left(z_{n} \pi_{f} t_{n}\right)_{n}$ possui uma subsequência que converge fortemente em $Z$.

Afirmamos que existe uma sequência crescente $\left(n_{k}\right)_{k}$ em $\mathbb{N}$ e, para todo $l \in \mathbb{Z} \operatorname{com} l \geq 0$, existe um $k_{0}(l) \in \mathbb{N}$ e um $w_{l} \in Z \operatorname{com}\left|w_{l}\right|_{Z} \leq C_{10}$ tal que $t_{n_{k}}-l \geq t_{\widetilde{N}}$ para $k \geq k_{0}(l)$ e tal que a sequência $\left(z_{n_{k}} \pi_{f}\left(t_{n_{k}}-l\right)\right)_{k}$ converge para $w_{l}$ fracamente em $Z$ e fortemente em $Y$.

Sem perda de generalidade podemos assumir que $t_{n} \geq t_{\widetilde{N}}$ para todo $n \in \mathbb{N}$. Logo,

$$
\left|z_{n} \pi_{f} t_{n}\right| \leq C_{10}, \text { para todo } n \in \mathbb{N} \text {. }
$$


Portanto, existe um $w_{0} \in Z$ e uma sequência $\left(m_{k}\right)_{k}$ em $\mathbb{N}$ tal que $t_{m_{k}} \rightarrow \infty$ quando $k \rightarrow \infty$ e

$$
\left(z_{m_{k}} \pi_{f} t_{m_{k}}\right)_{k} \text { converge para } w_{0} \text { fracamente em } Z \text { e fortemente em } Y \text {. }
$$

Defina $k_{0}(0)=m_{1}$. Como $t_{m_{k}} \rightarrow \infty$ quando $k \rightarrow \infty$, existe um $k_{0}(1) \in \mathbb{N}$ tal que $t_{m_{k}} \geq 1+t_{\widetilde{N}}$ para todo $k \geq k_{0}(1)$. Logo, $t_{m_{k}}-1 \geq t_{\widetilde{N}}$ para todo $k \geq k_{0}(1) \mathrm{e}$

$$
\left|z_{m_{k}} \pi_{f}\left(t_{m_{k}}-1\right)\right| \leq C_{10}, \text { para todo } k \geq k_{0}(1)
$$

Portanto, existe um $w_{1} \in Z$ e uma subsequência $\left(m_{k}^{1}\right)_{k}$ de $\left(m_{k}\right)_{k}$ tal que $t_{m_{k}^{1}} \rightarrow \infty$ quando $k \rightarrow \infty$ e

$$
\left(z_{m_{k}^{1}} \pi_{f}\left(t_{m_{k}^{1}}-1\right)\right)_{k} \text { converge para } w_{1} \text { fracamente em } Z \text { e fortemente em } Y .
$$

Procedendo recursivamente, para cada $l \in \mathbb{Z} \operatorname{com} l \geq 0$, existem um $k_{0}(l) \in \mathbb{N}$, uma sequência $\left(m_{k}^{l}\right)_{k}$, subsequência de $\left(m_{k}^{l-1}\right)_{k}$, e $w_{l} \in Z$ tais que $t_{m_{k}^{l-1}} \geq l+t_{\widetilde{N}}$ para todo $k \geq k_{0}(l) \mathrm{e}$

$$
\left(z_{m_{k}^{l}} \pi_{f}\left(t_{m_{k}^{l}}-l\right)\right)_{k} \text { converge para } w_{l} \text { fracamente em } Z \text { e fortemente em } Y \text {. }
$$

Para cada $k \in \mathbb{N}$, defina $n_{k}=m_{k}^{k}$. Fixe $l \in \mathbb{Z} \operatorname{com} l \geq 0$. Temos que $\left(n_{k}\right)_{k \geq k_{0}(l)}$ é uma subsequência de $\left(m_{k}^{l}\right)_{k}$ e portanto

$$
\left(z_{n_{k}} \pi_{f}\left(t_{n_{k}}-l\right)\right)_{k} \text { converge para } w_{l} \text { fracamente em } Z \text { e fortemente em } Y \text {. }
$$

e a afirmativa está demonstrada. Agora uma aplicação da Proposição 4.18 implica que para todo $l \in \mathbb{N}$ e $t \in[0, \infty)$,

$$
\left(z_{n_{k}} \pi_{f}\left(t_{n_{k}}-l\right)\right) \pi_{f} t \rightarrow w_{l} \pi_{f} t, \text { quando } k \rightarrow \infty, \text { fortemente em } Y \text {. }
$$

Portanto, $w_{l} \pi_{f} l=w_{0}$, para todo $l \in \mathbb{N}$. Defina a função $\mathscr{F}: Z \rightarrow \mathbb{R}$ por

$$
\mathscr{F}(z)=V(z)-V^{*}(z), z \in Z
$$

onde $V$ e $V^{*}$ são definidos como na Proposição $4.2 \operatorname{com} \gamma \equiv 1$ e $\delta \in[0, \infty)$ tal que $\lambda_{1}-\delta \alpha_{1}>0$ e $\alpha_{0}-2 \delta \varepsilon \geq 0$. Afirmamos que existe uma constante $C_{11} \in[0, \infty)$ tal que

$$
\sup \left\{\left.|\mathscr{F}(z)||z \in Z,| z\right|_{Z} \leq C_{10}\right\} \leq C_{11}
$$


De fato, seja $z \in Z \operatorname{com}|z|_{Z} \leq C_{10}$. Temos que

$$
\begin{aligned}
|\mathscr{F}(z)| & \leq|V(z)|+\left|V^{*}(z)\right| \\
& \leq \frac{1}{2} \int_{\Omega} \varepsilon\left|\delta z_{1}+z_{2}\right|^{2} d x+\frac{1}{2} \int_{\Omega}\left(\left(A \nabla z_{1}\right) \cdot \nabla z_{1}+\beta\left|z_{1}\right|^{2}-\delta \alpha\left|z_{1}\right|^{2}\right) d x \\
& +\frac{1}{2} \int_{\Omega} \delta^{2} \varepsilon\left|z_{1}\right|^{2} d x+\int_{\Omega}\left|\widehat{F}\left(z_{1}\right)\right| d x \\
& \leq \varepsilon\left(\left|\delta z_{1}\right|_{L^{2}(\Omega)}^{2}+\left|z_{2}\right|_{L^{2}(\Omega)}^{2}\right)+\frac{1}{2}\left(\lambda_{1}-\alpha_{0}+\delta^{2} \varepsilon\right)\left|z_{1}\right|_{L^{2}(\Omega)}^{2} \\
& +\bar{C}\left(\left.\left.\frac{1}{2}|a| z_{1}\right|^{2}\right|_{L^{1}(\Omega)}+\frac{1}{\bar{\rho}+2}\left|z_{1}\right|_{L^{\bar{\rho}+2}}^{\bar{\rho}+2}\right)+\left|z_{1}\right|_{L^{2}(\Omega)}|\widehat{f}(0)|_{L^{2}(\Omega)} .
\end{aligned}
$$

Aqui, usamos a desigualdade 3.10. A Hipótese 4.15 e o Teorema 1.10 completam a demonstração da afirmativa. Notemos que

$$
\mathscr{F}(z)=2\|z\|^{2}-V^{*}(z), z \in Z,
$$

onde $\|\cdot\|$ é a norma definida no Lema 4.20 .

Seja $\zeta(\cdot)=\left(\zeta_{1}(\cdot), \zeta_{2}(\cdot)\right):[0, \infty) \rightarrow Z$ uma solução arbitrária de $\pi_{f}$. A Proposição 4.2 implica que a função $\mathscr{F} \circ \zeta$ é continuamente diferenciável e, para todo $t \in[0, \infty)$,

$$
\begin{aligned}
(\mathscr{F} \circ \zeta)^{\prime}(t)+2 \delta(\mathscr{F} \circ \zeta)(t) & =\int_{\Omega}(2 \delta \varepsilon-\alpha(x))\left(\delta \zeta_{1}(t)(x)+\zeta_{2}(t)(x)\right)^{2} d x \\
& +\int_{\Omega} \delta \zeta_{1}(t)(x) f\left(x, \zeta_{1}(t)(x)\right) d x-2 \delta \int_{\Omega} F\left(x, \zeta_{1}(t)(x)\right) d x .
\end{aligned}
$$

Segue que para todo $t \in[0, \infty)$

$$
\begin{aligned}
(\mathscr{F}(\zeta(t)) & =e^{-2 \delta t} \mathscr{F}(\zeta(0))+\int_{0}^{t} e^{-2 \delta(t-s)}\left(\int_{\Omega}(2 \delta \varepsilon-\alpha(x))\left(\delta \zeta_{1}(s)(x)+\zeta_{2}(s)(x)\right)^{2} d x\right) d s \\
& +\int_{0}^{t} e^{-2 \delta(t-s)}\left(\int_{\Omega} \delta \zeta_{1}(s)(x) f\left(x, \zeta_{1}(s)(x)\right) d x-2 \delta \int_{\Omega} F\left(x, \zeta_{1}(s)(x)\right) d x\right) d s .
\end{aligned}
$$

Fixemos $l \in \mathbb{N}$ e, para $k \geq k_{0}(l)$, seja $\zeta_{k}(t)=\left(z_{n_{k}} \pi_{f}\left(t_{n_{k}}-l\right)\right) \pi_{f} t$ e $\zeta(t)=w_{l} \pi_{f} t$ para $t \in[0, \infty)$. Fazendo $t=l$ em 4.53) obtemos

$$
\begin{aligned}
2\left\|z_{n_{k}} \pi_{f} t_{n_{k}}\right\|^{2} & -V^{*}\left(z_{n_{k}} \pi_{f} t_{n_{k}}\right)=e^{-2 \delta l} \mathscr{F}\left(z_{n_{k}} \pi_{f}\left(t_{n_{k}}-l\right)\right) \\
& +\int_{0}^{l} e^{-2 \delta(l-s)}\left(\int_{\Omega}(2 \delta \varepsilon-\alpha(x))\left(\delta \zeta_{k, 1}(s)(x)+\zeta_{k, 2}(s)(x)\right)^{2} d x\right) d s \\
& +\int_{0}^{t} e^{-2 \delta(l-s)} \rho(s) d s,
\end{aligned}
$$


onde $\rho(s)=\int_{\Omega} \delta \zeta_{k, 1}(s)(x) f\left(x, \zeta_{k, 1}(s)(x)\right) d x-2 \delta \int_{\Omega} F\left(x, \zeta_{k, 1}(s)(x)\right) d x$, para $s \in[0, l] \mathrm{e}$

$$
\begin{aligned}
2\left\|w_{0}\right\|^{2} & -V^{*}\left(w_{0}\right)=e^{-2 \delta l} \mathscr{F}\left(w_{l}\right) \\
& +\int_{0}^{l} e^{-2 \delta(l-s)}\left(\int_{\Omega}(2 \delta \varepsilon-\alpha(x))\left(\delta \zeta_{1}(s)(x)+\zeta_{2}(s)(x)\right)^{2} d x\right) d s \\
& +\int_{0}^{l} e^{-2 \delta(l-s)}\left(\int_{\Omega} \delta \zeta_{1}(s)(x) f\left(x, \zeta_{1}(s)(x)\right) d x-2 \delta \int_{\Omega} F\left(x, \zeta_{1}(s)(x)\right) d x\right) d s .
\end{aligned}
$$

Afirmamos agora que

$$
V^{*}\left(z_{n_{k}} \pi_{f} t_{n_{k}}\right) \rightarrow V^{*}\left(w_{0}\right)
$$

e

$$
\begin{aligned}
\int_{0}^{l} e^{-2 \delta(l-s)} & \left(\int_{\Omega} \delta \zeta_{k, 1}(s)(x) f\left(x, \zeta_{k, 1}(s)(x)\right) d x-2 \delta \int_{\Omega} F\left(x, \zeta_{k, 1}(s)(x)\right) d x\right) d s \\
& \rightarrow \int_{0}^{l} e^{-2 \delta(l-s)}\left(\int_{\Omega} \delta \zeta_{1}(s)(x) f\left(x, \zeta_{1}(s)(x)\right) d x-2 \delta \int_{\Omega} F\left(x, \zeta_{1}(s)(x)\right) d x\right) d s
\end{aligned}
$$

quando $k \rightarrow \infty$. De fato, como o operador de $H_{0}^{1}(\Omega)$ em $L^{2}(\Omega)$ induzido por $u \mapsto|a| u$ é limitado, temos que existe uma constante $\widetilde{L}_{a} \in(0, \infty)$ tal que

$$
|| a|u|_{L^{2}(\Omega)} \leq \widetilde{L}_{a}|u|_{H_{0}^{1}(\Omega)}, \text { para todo } u \in H_{0}^{1}(\Omega)
$$

Este fato, a desigualdade (3.11) da Proposição 3.8 e o Teorema 1.10 implicam que

$$
\begin{aligned}
& \left|V^{*}\left(z_{n_{k}} \pi_{f} t_{n_{k}}\right)-V^{*}\left(w_{0}\right)\right|=\left|V^{*}\left(\zeta_{k, 1}(l)\right)-V^{*}\left(\zeta_{1}(l)\right)\right| \\
& \leq \int_{\Omega}\left|\widehat{F}\left(\zeta_{k, 1}(l)\right)-\widehat{F}\left(\zeta_{1}(l)\right)\right| d x \\
& \leq\left[|\widehat{f}(0)|_{L^{2}(\Omega)}+\bar{C}\left(\left|a \zeta_{k, 1}(l)\right|_{L^{2}(\Omega)}+\left|a\left(\zeta_{k, 1}(l)-\zeta_{1}(l)\right)\right|_{L^{2}(\Omega)}\right)\right. \\
& \left.+\bar{C} \max \left(1,2^{\bar{\rho}}\right)\left(\left|\zeta_{k, 1}(l)\right|_{L^{2(\bar{\rho}+1)}(\Omega)}^{\bar{\rho}+1}+\left|\zeta_{k, 1}(l)-\zeta_{1}(l)\right|_{L^{2(\bar{\rho}+1)}(\Omega)}^{\bar{\rho}+1}\right)\right]\left|\zeta_{k, 1}(l)-\zeta_{1}(l)\right|_{L^{2}(\Omega)} \\
& \leq\left[|\widehat{f}(0)|_{L^{2}(\Omega)}+\bar{C}\left(\widetilde{L}_{a}\left|\zeta_{k, 1}(l)\right|_{H_{0}^{1}(\Omega)}+\left.\widetilde{L}_{a}\left(\mid \zeta_{k, 1}(l)-\zeta_{1}(l)\right)\right|_{H_{0}^{1}(\Omega)}\right)\right. \\
& \left.+\bar{C} \max \left(1,2^{\bar{\rho}}\right)\left(K_{2(\bar{\rho}+1)}^{\bar{\rho}+1}\left|\zeta_{k, 1}(l)\right|_{H_{0}^{1}(\Omega)}^{\bar{\rho}+1}+K_{2(\bar{\rho}+1)}^{\bar{\rho}+1}\left|\zeta_{k, 1}(l)-\zeta_{1}(l)\right|_{H_{0}^{1}(\Omega)}^{\bar{\rho}+1}\right)\right]\left|\zeta_{k, 1}(l)-\zeta_{1}(l)\right|_{L^{2}(\Omega)} .
\end{aligned}
$$

Como

$$
\left|\zeta_{k, 1}(l)-\zeta_{1}(l)\right|_{L^{2}(\Omega)} \rightarrow 0, \text { quando } k \rightarrow \infty
$$


obtemos 4.56). Para provar 4.57, notemos que

$$
\begin{aligned}
\mid \int_{0}^{l} e^{-2 \delta(l-s)} & \left(\int_{\Omega} \delta \zeta_{k, 1}(s)(x) f\left(x, \zeta_{k, 1}(s)(x)\right) d x-2 \delta \int_{\Omega} F\left(x, \zeta_{k, 1}(s)(x)\right) d x\right) d s \\
& -\int_{0}^{l} e^{-2 \delta(l-s)}\left(\int_{\Omega} \delta \zeta_{1}(s)(x) f\left(x, \zeta_{1}(s)(x)\right) d x-2 \delta \int_{\Omega} F\left(x, \zeta_{1}(s)(x)\right) d x\right) d s \mid \\
& \leq \int_{0}^{l} e^{-2 \delta(l-s)}\left(\left|\int_{\Omega} \delta \zeta_{k, 1}(s)(x) f\left(x, \zeta_{k, 1}(s)(x)\right)-\zeta_{1}(s)(x) f\left(x, \zeta_{1}(s)(x)\right) d x\right|\right) d s \\
& +2 \delta \int_{0}^{l} \int_{\Omega}\left|F\left(x, \zeta_{k, 1}(s)(x)\right)-F\left(x, \zeta_{1}(s)(x)\right)\right| d x d s .
\end{aligned}
$$

Como para todo para $s \in[0, l]$,

$$
\left|\zeta_{1}(s)-\zeta_{k, 1}(s)\right|_{L^{2}(\Omega)} \rightarrow 0, \text { quando } k \rightarrow \infty
$$

e o intervalo $[0, l]$ é compacto, a desigualdade (3.11) da Proposição 3.8 e 4.58) implicam que dado $\eta>0$, existe um $k_{1} \in \mathbb{N}$ tal que

$$
\int_{\Omega}\left|F\left(x, \zeta_{k, 1}(s)(x)\right)-F\left(x, \zeta_{1}(s)(x)\right)\right| d x<\eta, \text { para todo } k \geq k_{1} \text { e para todo } s \in[0, l]
$$

Por outro lado, a desigualdade (3.13) da Proposição 3.8 implica que

$$
\begin{aligned}
\mid \int_{\Omega} \zeta_{k, 1} & (s)(x) \widehat{f}\left(\zeta_{k, 1}(s)\right)-\zeta_{1}(s)(x) \widehat{f}\left(\zeta_{1}(s)\right) d x \mid \\
& =\left|\int_{\Omega} \zeta_{k, 1}(s)(x)\left(\widehat{f}\left(\zeta_{k, 1}(s)\right)-\widehat{f}\left(\zeta_{1}(s)\right)\right)+\left(\zeta_{k, 1}(s)(x)-\zeta_{1}(s)(x)\right) \widehat{f}\left(\zeta_{1}(s)\right)\right| d x \\
& =\left|\left\langle\left(\zeta_{k, 1}(s)-\zeta_{1}(s)\right), \widehat{f}\left(\zeta_{1}(s)\right)\right\rangle-\left\langle\zeta_{k, 1}(s),\left(\widehat{f}\left(\zeta_{k, 1}(s)\right)-\widehat{f}\left(\zeta_{1}(s)\right)\right)\right\rangle\right| \\
& \leq\left|\left\langle\zeta_{k, 1}(s)-\zeta_{1}(s), \widehat{f}\left(\zeta_{1}(s)\right)\right\rangle\right|+\left|\left\langle\zeta_{k, 1}(s), \widehat{f}\left(\zeta_{k, 1}(s)\right)-\widehat{f}\left(\zeta_{1}(s)\right)\right\rangle\right| \\
& \leq\left|\widehat{f}\left(\zeta_{1}(s)\right)\right|_{H^{-1}(\Omega)}\left|\zeta_{k, 1}(s)-\zeta_{1}(s)\right|_{L^{2}(\Omega)}+\left|\zeta_{k, 1}(s)\right|_{L^{2}(\Omega)}\left|\widehat{f}\left(\zeta_{k, 1}(s)\right)-\widehat{f}\left(\zeta_{1}(s)\right)\right|_{H^{-1}(\Omega)}
\end{aligned}
$$

onde $s \in[0, l]$. Como anteriormente, usando (3.13), temos que dado $\eta>0$, existe um $k_{2} \in \mathbb{N}$ com $k_{2} \geq k_{1}$ tal que

$$
\left|\int_{\Omega} \zeta_{k, 1}(s)(x) \widehat{f}\left(\zeta_{k, 1}(s)\right)-\zeta_{1}(s)(x) \widehat{f}\left(\zeta_{1}(s)\right) d x\right| \leq \eta, \text { para } k \geq k_{2} \text { e } s \in[0, l]
$$


Usando desigualdades 4.61) e 4.62) em 4.59) obtemos

$$
\begin{aligned}
& \mid \int_{0}^{l} e^{-2 \delta(l-s)}\left(\int_{\Omega} \delta \zeta_{k, 1}(s)(x) f\left(x, \zeta_{k, 1}(s)(x)\right) d x-2 \delta \int_{\Omega} F\left(x, \zeta_{k, 1}(s)(x)\right) d x\right) d s \\
& -\int_{0}^{l} e^{-2 \delta(l-s)}\left(\int_{\Omega} \delta \zeta_{1}(s)(x) f\left(x, \zeta_{1}(s)(x)\right) d x-2 \delta \int_{\Omega} F\left(x, \zeta_{1}(s)(x)\right) d x\right) d s \mid \\
& \leq \int_{0}^{l} e^{-2 \delta(l-s)} \eta d s=\frac{\eta}{2 \delta} .
\end{aligned}
$$

Como $\eta>0$ arbitrário, a convergência em 4.57) está demonstrada. A próxima afirmativa é que

$$
\begin{aligned}
\limsup _{k \rightarrow \infty} & \int_{0}^{l} e^{-2 \delta(l-s)}\left(\int_{\Omega}(2 \delta \varepsilon-\alpha(x))\left(\delta \zeta_{k, 1}(s)(x)+\zeta_{k, 2}(s)(x)\right)^{2} d x\right) d s \\
\leq & \int_{0}^{l} e^{-2 \delta(l-s)}\left(\int_{\Omega}(2 \delta \varepsilon-\alpha(x))\left(\delta \zeta_{1}(s)(x)+\zeta_{2}(s)(x)\right)^{2} d x\right) d s
\end{aligned}
$$

De fato, como $\alpha(x)-2 \delta \varepsilon \leq 0$ para todo $x \in \Omega$, temos pelo Lema de Fatou que

$$
\begin{aligned}
\limsup _{k \rightarrow \infty} & \int_{0}^{l} e^{-2 \delta(l-s)}\left(\int_{\Omega}(2 \delta \varepsilon-\alpha(x))\left(\delta \zeta_{k, 1}(s)(x)+\zeta_{k, 2}(s)(x)\right)^{2} d x\right) d s \\
& =-\liminf _{k \rightarrow \infty} \int_{0}^{l} e^{-2 \delta(l-s)}\left(\int_{\Omega}(\alpha(x)-2 \delta \varepsilon)\left(\delta \zeta_{k, 1}(s)(x)+\zeta_{k, 2}(s)(x)\right)^{2} d x\right) d s \\
\leq & -\int_{0}^{l} e^{-2 \delta(l-s)} \liminf _{k \rightarrow \infty}\left(\int_{\Omega}(\alpha(x)-2 \delta \varepsilon)\left(\delta \zeta_{k, 1}(s)(x)+\zeta_{k, 2}(s)(x)\right)^{2} d x\right) d s
\end{aligned}
$$

Seja $s \in[0, l]$ arbitrário. Como a sequência $\left(\left(\zeta_{k, 1}(s), \zeta_{k, 2}(s)\right)\right)_{k}$ converge fracamente em $Z$ para $\left(\zeta_{1}(s), \zeta_{2}(s)\right)$ e $\Psi$ é uma aplicação contínua e linear, temos que $\Psi$ é também fracamente contínua e, portanto, $\left(\left(\zeta_{k, 1}(s), \delta \zeta_{k, 1}(s)+\zeta_{k, 2}(s)\right)\right)_{k}$ converge fracamente em $Z$ para $\left(\zeta_{1}(s), \delta \zeta_{1}(s)+\zeta_{2}(s)\right)$. Assim, para todo $v \in L^{2}(\Omega)$,

$$
\left\langle v, \delta \zeta_{k, 1}(s)+\zeta_{k, 2}(s)\right\rangle \rightarrow\left\langle v, \delta \zeta_{1}(s)+\zeta_{2}(s)\right\rangle, \text { quando } k \rightarrow \infty
$$

Tomando $v=(\alpha-2 \delta \varepsilon)\left(\delta \zeta_{1}(s)+\zeta_{2}(s)\right)$, obtemos

$$
\begin{aligned}
\mid(\alpha & -2 \delta \varepsilon)\left.^{1 / 2}\left(\delta \zeta_{1}(s)+\delta \zeta_{2}(s)\right)\right|_{L^{2}(\Omega)} ^{2} \\
& =\left\langle(\alpha-2 \delta \varepsilon)^{1 / 2}\left(\delta \zeta_{1}(s)+\delta \zeta_{2}(s)\right),(\alpha-2 \delta \varepsilon)^{1 / 2}\left(\delta \zeta_{1}(s)+\delta \zeta_{2}(s)\right)\right\rangle \\
& =\lim _{k \rightarrow \infty}\left\langle(\alpha-2 \delta \varepsilon)^{1 / 2}\left(\delta \zeta_{1}(s)+\delta \zeta_{2}(s)\right),(\alpha-2 \delta \varepsilon)^{1 / 2}\left(\delta \zeta_{k, 1}(s)+\delta \zeta_{k, 2}(s)\right)\right\rangle \\
& \leq\left|(\alpha-2 \delta \varepsilon)^{1 / 2}\left(\delta \zeta_{1}(s)+\delta \zeta_{2}(s)\right)\right|_{L^{2}(\Omega)} \liminf _{k \rightarrow \infty}\left|(\alpha-2 \delta \varepsilon)^{1 / 2}\left(\delta \zeta_{k, 1}(s)+\delta \zeta_{k, 2}(s)\right)\right|_{L^{2}(\Omega)}
\end{aligned}
$$


Portanto,

$$
\left|(\alpha-2 \delta \varepsilon)^{1 / 2}\left(\delta \zeta_{1}(s)+\delta \zeta_{2}(s)\right)\right|_{L^{2}(\Omega)} \leq \liminf _{k \rightarrow \infty}\left|(\alpha-2 \delta \varepsilon)^{1 / 2}\left(\delta \zeta_{k, 1}(s)+\delta \zeta_{k, 2}(s)\right)\right|_{L^{2}(\Omega)}
$$

e, assim,

$$
\left(\int_{\Omega}(\alpha-2 \delta \varepsilon)\left(\delta \zeta_{1}(s)+\delta \zeta_{2}(s)\right)^{2} d x\right) \leq \liminf _{k \rightarrow \infty}\left(\int_{\Omega}(\alpha-2 \delta \varepsilon)^{1 / 2}\left(\delta \zeta_{k, 1}(s)+\delta \zeta_{k, 2}(s)\right) d x\right) .
$$

A afirmativa em (4.63) é uma consequência das desigualdades 4.65) e 4.64). Usando as desigualdades 4.52), 4.54)-4.57) e 4.63) obtemos

$$
\begin{aligned}
\limsup _{k \rightarrow \infty} & 2\left\|z_{n_{k}} \pi_{f} t_{n_{k}}\right\|^{2}-V^{*}\left(w_{0}\right) \leq e^{2 \delta l} C_{11} \\
& +\int_{0}^{l} e^{-2 \delta(l-s)}\left(\int_{\Omega}(2 \delta \varepsilon-\alpha(x))\left(\delta \zeta_{1}(s)(x)+\zeta_{2}(s)(x)\right)^{2} d x\right) d s \\
& +\int_{0}^{l} e^{-2 \delta(l-s)}\left(\int_{\Omega} \delta \zeta_{1}(s)(x) f\left(x, \zeta_{1}(s)(x)\right) d x-2 \delta \int_{\Omega} F\left(x, \zeta_{1}(s)(x)\right) d x\right) d s \\
& =e^{-2 \delta l} C_{11}+2\left\|w_{0}\right\|^{2}-V^{*}\left(w_{0}\right)-e^{2 \delta l} \mathscr{F}\left(w_{l}\right) \\
& \leq 2 e^{-2 \delta l} C_{11}+2\left\|w_{0}\right\|^{2}-V^{*}\left(w_{0}\right) .
\end{aligned}
$$

Portanto, para todo $l \in \mathbb{N}$,

$$
\underset{k \rightarrow \infty}{\limsup } 2\left\|z_{n_{k}} \pi_{f} t_{n_{k}}\right\|^{2} \leq 2 e^{-2 \delta l} C_{11}+2\left\|w_{0}\right\|^{2}
$$

e, assim, $\lim \sup _{k \rightarrow \infty}\left\|z_{n_{k}} \pi_{f} t_{n_{k}}\right\| \leq\left\|w_{0}\right\|$. Como $\left(z_{n_{k}} \pi_{f} t_{n_{k}}\right)_{k}$ converge fracamente para $w_{0}$ em $(Z,[\cdot, \cdot])$, temos

$$
\liminf _{k \rightarrow \infty}\left\|z_{n_{k}} \pi_{f} t_{n_{k}}\right\| \geq\left\|w_{0}\right\|
$$

Obtemos

$$
\lim _{k \rightarrow \infty}\left\|z_{n_{k}} \pi_{f} t_{n_{k}}\right\|=\left\|w_{0}\right\|
$$

O Lema 4.20 implica que $\left(z_{n_{k}} \pi_{f} t_{n_{k}}\right)_{k}$ converge para $w_{0}$ fortemente em $Z$. A demonstração do teorema está completa.

Portanto, temos o seguinte resultado de existência de atrator global para o caso crítico.

Teorema 4.21. Assuma a Hipótese 3.9 a Hipótese 4.3 e a Hipótese 4.15. O semifluxo $\pi_{f}$ é um semifluxo global e possui um atrator global. 
Demonstração. Isso é uma consequência imediata do Teorema 4.12, do Teorema 4.19 e da Proposição 1.60 . 


\section{Considerações finais}

Observamos que a equação $(E)$ depende do parâmetro $\varepsilon>0$. Portanto, o semifluxo global $\pi_{f}$ depende de $\varepsilon>0$, ou seja, $\pi_{f}=\pi_{f, \varepsilon}$. Denote por $\mathscr{A}_{\varepsilon}$ o atrator global de $\pi_{f, \varepsilon}$. Observamos que as estimativas de truncamento para a solução $u(x, t)$ descritas no Capítulo 4 são uniformes no parâmetro $\varepsilon>0$. Isto nos coloca a pergunta sobre o que ocorre com a família $\left(\mathscr{A}_{\mathcal{E}}\right)_{\varepsilon>0}$ quando $\varepsilon \rightarrow 0$.

Assuma $\alpha \equiv 1 \mathrm{em}(E)$. Podemos fazer, formalmente, $\varepsilon=0 \mathrm{em}(E)$ e obtemos a equação de reação-difusão

$$
\begin{array}{ll}
u_{t}+\beta(x) u-\sum_{i, j} \partial_{i}\left(a_{i j}(x) \partial_{j} u\right)=f(x, u), & x \in \Omega, t \in[0, \infty), \\
u(x, t)=0, & x \in \partial \Omega, t \in[0, \infty) .
\end{array}
$$

Em [26], os autores estudam o caso $N=3$ e mostram que sob certas condições o problema $(P)$ possui atrator global $\widetilde{\mathscr{A}}$ em $H_{0}^{1}(\Omega)$.

Em [24] os autores definem uma imersão $\Gamma: D \rightarrow H_{0}^{1}(\Omega) \times L^{2}(\Omega)$, onde $D$ é um subconjunto de $H_{0}^{1}(\Omega)$, mostram que $\widetilde{\mathscr{A}} \subset D$ e que a família $\left(\mathscr{A}_{\varepsilon}\right)_{\varepsilon \geq 0}$, onde $\mathscr{A}_{0}=\Gamma(\widetilde{\mathscr{A}})$, é semicontínua superiormente em $\varepsilon=0$. 


\section{Demonstrações auxiliares}

Nesse apêndice apresentaremos as demonstrações de alguns resultados do Capítulo 1.

\section{A.1 Demonstração de resultados da Seção 1.5}

Para demonstrar a Proposição 1.51 necessitamos do seguinte resultado auxiliar.

Lema A.1. Suponha que $\pi$ seja um semifluxo global assintoticamente compacto e seja $B$ um subconjunto não-vazio de $X$ tal que $\gamma_{t_{0}}^{+}(B)$ é um conjunto limitado, para algum $t_{0} \in \mathbb{R}^{+}$. Então $\omega(B)$ é um conjunto não-vazio, compacto, invariante. Além disso $\omega(B)$ atrai $B$.

Demonstração da Proposição 1.51 Suponha que $\pi$ seja assintoticamente suave. Mostremos que $\pi$ é assintoticamente compacto. De fato, seja $B$ um subconjunto u-limitado de $X,\left(x_{n}\right)_{n}$ um sequência em $B$ e $\left(t_{n}\right)_{n}$ um sequência em $[0, \infty), \operatorname{com} t_{n} \rightarrow \infty$ quando $n \rightarrow \infty$.

Logo existe um $t_{B} \geq 0$ tal que o conjunto $\gamma_{t_{B}}^{+}(B)$ é limitado. Segue do Lema A.1 que $\omega(B)$ é não-vazio, compacto, invariante e atrai $B$. Em particular temos que $\operatorname{dist}_{H}\left(\pi_{\left(t_{n}\right)}(B), \omega(B)\right) \rightarrow 0$ quando $n \rightarrow \infty$. Como

$$
d\left(x_{n} \pi t_{n}, \omega(B)\right) \leq \operatorname{dist}_{H}\left(\pi_{\left(t_{n}\right)}(B), \omega(B)\right), \text { para todo } n \in \mathbb{N}
$$

temos que $d\left(x_{n} \pi t_{n}, \omega(B)\right) \rightarrow 0$ quando $n \rightarrow \infty$. A compacidade de $\omega(B)$ implica que existe uma subsequência $\left(x_{n_{k}} \pi t_{n_{k}}\right)_{k}$ da sequência $\left(x_{n} \pi t_{n}\right)_{n}$ e um $y \in \omega(B)$ tais que $x_{n_{k}} \pi t_{n_{k}} \rightarrow y$ quando $k \rightarrow \infty$. Isso implica que $\pi$ é assintoticamente compacto.

Reciprocamente, suponha que $\pi$ seja assintoticamente compacto e considere um subconjunto $B$ de $X$ não-vazio, fechado, limitado e positivamente invariante. Afirmamos que $\omega(B)$ é subconjunto de $B$ não-vazio, invariante, compacto e que $\omega(B)$ atrai $B$. 
Mostremos que $\omega(B)$ é não-vazio. De fato, seja $v \in B$ e considere o conjunto $\{v\}$. Como $B$ é positivamente invariante, temos que, temos que $v \pi t \in B$, para todo $t \geq 0$. Ou seja, $\{v\}$ é u-limitado com $t_{\{v\}}=0$. Seja $\left(t_{n}\right)_{n}$ uma sequência em $\mathbb{N}$ tal que $t_{n} \rightarrow \infty$ quando $n \rightarrow \infty$. Como $\pi$ é assintoticamente compacto, segue que existe uma subquência $\left(v \pi t_{n_{k}}\right)_{k}$, da sequência $\left(v \pi t_{n}\right)_{n}$, e $y \in X$ tal que $d\left(v \pi t_{n_{k}}, y\right) \rightarrow 0$ quando $k \rightarrow \infty$. Logo $y \in \omega(\{v\}) \subset \omega(B)$.

Mostremos que $\omega(B) \subset B$. De fato, dado $y \in \omega(B)$ existem sequências $\left(x_{n}\right)_{n}$ em $B$ e $\left(t_{n}\right)_{n}$ em $\mathbb{N}$ tais que $t_{n} \rightarrow \infty$ e $d\left(x_{n} \pi t_{n}, y\right) \rightarrow 0$ quando $n \rightarrow \infty$. Como $B$ é positivamente invariante, temos que $x_{n} \pi t_{n} \in B$ para todo $n \in \mathbb{N}$ e, sendo $B$ fechado, segue que $y \in B$.

Mostremos que $\omega(B)$ atrai $B$. De fato, suponha que $\omega(B)$ não atraia $B$. Então existem um $\varepsilon_{0}>0$, uma sequência $\left(t_{n}\right)_{n}$ em $[0, \infty) \operatorname{com} t_{n} \rightarrow \infty$ e uma sequência $\left(v_{n}\right)_{n}$ em $B$ tais que

$$
\inf _{y \in \omega(B)} d\left(v_{n} \pi t_{n}, y\right)>\varepsilon_{0}, \text { para todo } n \in \mathbb{N}
$$

Como $B$ é positivamente invariante e limitado, conjunto $E=\left\{v_{n} \pi t_{n} \mid n \in \mathbb{N}\right\} \subset B$ é u-limitado com $t_{E}=0$. Agora, a compacidade assintótica de $\pi$ implica que existe uma subsequência $\left(v_{n_{k}} \pi t_{n_{k}}\right)_{k}$ da sequência $\left(v_{n} \pi t_{n}\right)_{n}$ e um $z \in X$ tal que $d\left(v_{n_{k}} \pi t_{n_{k}}, z\right) \rightarrow 0$ quando $k \rightarrow \infty$. Logo $z \in \omega(\{v\}) \subset \omega(B)$ e segue de A.1

$$
d\left(v_{n_{k}} \pi t_{n_{k}}, z\right)>\varepsilon_{0}, \text { para todo } k \in \mathbb{N}
$$

o que é uma contradição. Logo, $\omega(B)$ atrai $B$.

Mostremos que $\omega(B)$ é invariante. Fixe $t \geq 0$ e considere $v \in \omega(B)$. Logo existem sequências $\left(v_{n}\right)_{n}$ em $B$ e $\left(t_{n}\right)_{n}$ em $\mathbb{N}$ tais que $t_{n} \rightarrow \infty$ e $d\left(v_{n} \pi t_{n}, v\right) \rightarrow 0$ quando $n \rightarrow \infty$.

A continuidade de $\pi$ implica que $d\left(\left(v_{n} \pi t_{n}\right) \pi t, v \pi t\right) \rightarrow 0$ quando $n \rightarrow \infty$. Como $\left(v_{n} \pi t_{n}\right) \pi t=$ $v_{n} \pi\left(t_{n}+t\right)$ para todo $n \in \mathbb{N}$ e $t_{n}+t \rightarrow \infty$ quando $n \rightarrow \infty$, temos que $v \pi t \in \omega(B)$. Ou seja,

$$
\{x \pi t \mid x \in \omega(B)\} \subset \omega(B) .
$$

Como $t_{n} \rightarrow \infty$ quando $n \rightarrow \infty$, temos que existe um $n_{0} \in \mathbb{N}$ tal que $t_{n} \geq t$ para todo $n \geq n_{0}$. Analogamente ao que já foi feito, o conjunto $\left\{v_{n} \pi\left(t_{n}-t\right) \mid n \geq n_{0}\right\}$ é u-limitado e a compacidade assintótica de $\pi$ implica que existe uma subsequência $\left(v_{n_{k}} \pi\left(t_{n_{k}}-t\right)\right)_{k}$ da sequência $\left(v_{n} \pi\left(t_{n}-\right.\right.$ $t)_{n \geq n_{0}}$ e um $z \in X$ tal que $d\left(v_{n_{k}} \pi\left(t_{n_{k}}-t\right), z\right) \rightarrow 0$ quando $k \rightarrow \infty$. Novamente $z \in \omega(B)$ e a continuidade do semifluxo $\pi$ implica que $d\left(\left(v_{n_{k}} \pi\left(t_{n_{k}}-t\right)\right) \pi t, z \pi t\right) \rightarrow 0$ quando $k \rightarrow \infty$. Como $\left(v_{n_{k}} \pi\left(t_{n_{k}}-t\right)\right) \pi t=v_{n_{k}} \pi t$ para todo $k \in N$ temos que $v=z \pi t$. Ou seja

$$
\omega(B) \subset\{x \pi t \mid x \in \omega(B)\}
$$


Finalmente, mostremos que $\omega(B)$ é compacto. Notemos que $\omega(B)$ é u-limitado. A invariância de $\omega(B)$ e a compacidade assintótica de $\pi$ implicam que $\omega(B)$ é sequencialmente compacto. Portanto, $\pi$ é assintoticamente suave.

Demonstração do Lema 1.55 Seja $W$ um subconjunto limitado de $X$. Suponha que $\mathscr{A}$ não atraia $W$. Logo, temos que existem um $\varepsilon_{0}>0$ e uma sequência $\left(t_{n}\right)_{n}$ em $[0, \infty) \operatorname{com} t_{n} \rightarrow \infty$ quando $n \rightarrow \infty$ e uma sequência $\left(w_{n}\right)_{n}$ em $W$ tais que

$$
d\left(w_{n} \pi t_{n}, \mathscr{A}\right)>\varepsilon_{0}, \text { para todo } n \in \mathbb{N} \text {. }
$$

Portanto,

$$
d\left(w_{n} \pi t_{n}, a\right)>\varepsilon_{0}, \text { para todo } n \in \mathbb{N} \text { e todo } a \in \mathscr{A} .
$$

Notemos que $U:=\bigcup_{a \in \mathscr{A}} B_{X}\left(a, \varepsilon_{0}\right)$ é uma vizinhança a aberta de $\mathscr{A}$. Como $\mathscr{A}$ é um atrator global de $\pi$, existe um $t_{W, U} \in[0, \infty)$ tal que $w \pi t \in U$ para todo $w \in W$ e $t \in\left[t_{W, U}, \infty\right)$. Portanto, existe um $n_{0} \in \mathbb{N}$ tal que $t_{n} \geq t_{W, U}$ para todo $n \geq n_{0} \mathrm{e}$

$$
w_{n} \pi t_{n} \in \bigcup_{a \in \mathscr{A}} B_{X}\left(a, \varepsilon_{0}\right) \text { para todo } n \geq n_{0}
$$

Logo, existe uma sequência $\left(a_{n}\right)_{n}$ em $\mathscr{A}$ tal que

$$
d\left(w_{n} \pi t_{n}, a_{n}\right)<\varepsilon_{0} \text { para todo } n \geq n_{0} .
$$

Como $\mathscr{A}$ é um conjunto compacto, existe uma subsequência $\left(a_{n_{k}}\right)_{n_{k}}$ da sequência $\left(a_{n}\right)_{n}$ e um $a \in \mathscr{A}$ tais que $a_{n_{k}} \rightarrow a$ quando $k \rightarrow \infty$. Este fato e (A.3) implicam que existe um $k_{0} \in \mathbb{N}$ tal que

$$
d\left(w_{n_{k}} \pi t_{n_{k}}, a\right) \leq \varepsilon_{0} \text { para todo } k \geq k_{0}
$$

mas isso contradiz (A.2). Portanto, o conjunto $\mathscr{A}$ atrai conjuntos limitados de $X$.

Demonstração do Lema 1.56 Seja $\mathscr{A}$ um atrator global de $\pi$. Segue do Lema 1.55 que $\mathscr{A}$ atrai pontos de $X$, ou seja, $\pi$ é ponto dissipativo.

Mostremos que $\pi$ é assintoticamente suave. Seja $W$ um subconjunto não-vazio, fechado, limitado e positivamente invariante de $X$. Devemos mostrar que existe um conjunto compacto não-vazio $C$ contido em $W$ que atrai $W$. Defina $C:=W \cap \mathscr{A}$. É claro que $C$ limitado e $C \subset W$. Como $\mathscr{A}$ é compacto e $W$ é fechado, segue que $C$ é fechado. Além disso, $C \subset \mathscr{A}$. Portanto, $C$ é compacto. 
Afirmamos que $C$ é não vazio. De fato, seja $\left(t_{n}\right)_{n}$ uma sequência em $[0, \infty)$ com $t_{n} \rightarrow \infty$ quando $n \rightarrow \infty$ e $w \in W$. Segue do Lema 1.55 que $\mathscr{A}$ atrai $W$. Em particular, $\operatorname{dist}_{H}\left(\pi_{\left(t_{n}\right)}(W), \mathscr{A}\right) \rightarrow 0$ quando $n \rightarrow \infty$. Como

$$
d\left(w \pi t_{n}, \mathscr{A}\right) \leq \operatorname{dist}_{H}\left(\pi_{\left(t_{n}\right)}(W), \mathscr{A}\right), \text { para todo } n \in \mathbb{N},
$$

temos que $d\left(w \pi t_{n}, \mathscr{A}\right) \rightarrow 0$ quando $n \rightarrow \infty$. A compacidade de $\mathscr{A}$ implica que existe uma subequência $\left(t_{n_{k}}\right)_{k}$ da sequência $\left(t_{n}\right)_{n}$ e um $y \in \mathscr{A}$ tais que $w \pi t_{n_{k}} \rightarrow y$ quando $k \rightarrow \infty$. Como $W$ é positivamente invariante e fechado, segue que $y \in W$. Ou seja, $y \in C$. Como $\mathscr{A}$ atrai $W$ e

$$
\operatorname{dist}_{H}\left(\pi_{(t)}(W), \mathscr{A} \cap W\right) \leq \operatorname{dist}_{H}\left(\pi_{(t)}(W), \mathscr{A}\right), \text { para todo } t \geq 0,
$$

segue $C$ atrai $W$.

Para concluir a demonstração, mostremos que $\pi$ é eventualmente limitado. Seja $W$ um subconjunto limitado de $X$. Temos que $\mathscr{A}$ atrai $W$. Logo existe um $t_{W} \geq 0$ tal que

$$
\operatorname{dist}_{H}\left(\pi_{(t)}(W), \mathscr{A}\right)<1 \text {, para todo } t \geq t_{W}
$$

Portanto,

$$
d(w \pi t, \mathscr{A})<1, \text { para todo } t \geq t_{W} \text { e } w \in W .
$$

Como $\mathscr{A}$ é limitado, segue que existe um $z \in X$ e um $M \geq 0$ tal que $d(a, z) \leq M$ para todo $a \in \mathscr{A}$. Seja $t \geq t_{W}$ e $w \in W$. Segue de (A.4) que existe um $a_{t, w} \in \mathscr{A}$ tal que $d\left(w \pi t, a_{t, w}\right)<1$. Portanto, temos que

$$
d(w \pi t, z) \leq 1+M, \text { para todo } w \in W \text { e } t \geq t_{W} .
$$

Ou seja, $\gamma_{t_{W}}^{+}(W)$ é limitado.

Demonstração do Lema 1.57 Como $\pi$ é um semifluxo ponto dissipativo, existe um subconjunto $W_{0}$ de $X$ não-vazio e limitado que atrai pontos de $X$. Seja $\mathscr{N}_{W_{0}}$ uma vizinhança aberta limitada arbitrária de $W_{0}$. Segue da Proposição 1.49 que $\mathscr{N}_{W_{0}}$ absorve pontos de $X$.

A continuidade de $\pi$ e o fato de que $\mathscr{N}_{W_{0}}$ absorve pontos implicam que para cada $v \in X$, existem um $\tau_{v} \geq 0$ e um $\varepsilon_{v}>0$ tais que

$$
\pi_{\left(\tau_{v}\right)}\left(B_{X}\left(v, \varepsilon_{v}\right)\right) \subset \mathscr{N}_{W_{0}},
$$

onde $B_{X}\left(v, \varepsilon_{v}\right)$ denota a bola aberta em $X$ com centro em $v$ e raio $\varepsilon_{v}$. 
Como $\pi$ é um semifluxo eventualmente limitado temos que existe um $t_{\mathscr{N}_{W_{0}}} \geq 0$ tal que

$$
\mathscr{O}:=\bigcup_{t \geq t_{\mathcal{N}_{W_{0}}}} \pi_{(t)}\left(\mathscr{N}_{W_{0}}\right)=\gamma_{t_{\mathcal{W}_{W_{0}}}}^{+}\left(\mathscr{N}_{W_{0}}\right)
$$

é um conjunto limitado. Segue que o conjunto $\mathscr{O}$ é positivamente invariante e absorve pontos de $X$. Além disso, segue de A.5 que para cada $v \in X$ temos que

$$
\pi_{(t)}\left(B_{X}\left(v, \varepsilon_{v}\right)\right) \subset \mathscr{O}, \text { para todo } t \geq t_{v},
$$

onde $t_{v}:=\tau_{v}+t_{\mathscr{N}_{W_{0}}} \geq 0$.

Seja $C$ um subconjunto compacto de $X$. É claro que $C \subset \cup_{v \in C} B_{X}\left(v, \varepsilon_{v}\right)$, onde $\varepsilon_{v}>0$ é como na afirmativa acima. A compacidade do conjunto $C$ implica que existem $v_{j} \in C, j=\{1, \ldots, k\}$ tais que

$$
C \subset \bigcup_{j=1}^{k} B_{X}\left(v_{i}, \varepsilon_{v_{j}}\right)=: \mathscr{N}_{C}
$$

Defina $\sigma:=\max \left\{t_{v_{1}}, \ldots, t_{v_{k}}\right\}$. Fórmulas A.6 e A.7 implicam que

$$
\pi_{(t)}(C) \subset \pi_{(t)}\left(\mathscr{N}_{C}\right)=\bigcup_{j=1}^{k} \pi_{(t)}\left(B_{X}\left(v_{j}, \varepsilon_{v_{j}}\right)\right) \subset \mathscr{O}, \text { para todo } t \geq \sigma .
$$

A demonstração está completa.

Demonstração do Lema 1.58 Seja $B$ um subconjunto limitado de $X$. O Lema A.1 implica que $\omega(B)$ é um conjunto compacto e que atrai $B$. Segue do Lema 1.57 que existe um conjunto limitado $\mathscr{O} \subset X$ e existe uma vizinhança aberta $\mathscr{N}_{\omega(B)}$ de $\omega(B)$ tal que $\mathscr{O}$ absorve $\mathscr{N}_{\omega(B)}$. Como $\omega(B)$ atrai $B$ temos que existe um $t_{B} \geq 0$ tal que

$$
\pi_{(t)}(B) \subset \mathscr{N}_{\omega(B)}, \text { para todo } t \geq t_{B}
$$

e portanto existe um $\tau_{B} \geq 0$ tal que

$$
\pi_{(t)}(B) \subset \mathscr{O}, \text { para todo } t \geq \tau_{B}
$$

Ou seja, mostramos que o conjunto $\mathscr{O}$ absorve conjuntos limitados de $X$.

Defina $\mathscr{A}=\omega(\mathscr{O})$. Aplicando novamente o Lema A.1 temos que $\mathscr{A}$ é um conjunto compacto, invariante e atrai $\mathscr{O}$. Além disso, como o conjunto $\mathscr{O}$ absorve conjuntos limitados de $X$, temos que $\mathscr{A}$ atrai subconjuntos limitados de $X$. Portanto, para todo conjunto limitado $B$ em $X$ 
e toda vizinhança aberta $U$ de $\mathscr{A}$, existe um $t_{B, U} \in[0, \infty)$ tal que $x \pi t \in U$ para todo $x \in B$ e todo $t \in\left[t_{B, U}, \infty\right)$. Mostramos que $\mathscr{A}$ é um atrator global do semifluxo $\pi$.

\section{A.2 Demonstração de resultados da Seção 1.6}

Demonstração da Proposição 1.62 Seja $\tau>0$. Defina $\alpha=\alpha(\tau)=\max \{\|T(t)\| \mid t \in[0, \tau]\}$, $K=2 M \alpha+\|F(0)\| \mathrm{e}$

$$
\omega_{M}=\omega_{M, \tau}=\min \left\{\tau, \frac{1}{\left(2 L_{K}+2\right) \alpha}\right\} .
$$

Seja agora $x \in X \operatorname{com}\|x\| \leq M$ e considere o seguinte espaço métrico:

$$
E=\left\{u \in C\left(\left[0, \omega_{M}\right], X\right) \mid\|u(t)\| \leq K, \text { para todo } t \in\left[0, \omega_{M}\right]\right\}
$$

munido da distância proveniente da norma de $C\left(\left[0, \omega_{M}\right], X\right)$, ou seja, para $u, v \in E$,

$$
d(u, v)=\max _{t \in\left[0, \omega_{M}\right]}\|u(t)-v(u)\|
$$

Temos que $(E, d)$ é um espaço métrico completo. Para cada $u \in E$, definimos a função $\Phi_{u}$ por

$$
\Phi_{u}(t)=T(t) x+\int_{0}^{t} T(t-s) F(u(s)) d s, \text { para todo } t \in\left[0, \omega_{M}\right]
$$

Notemos que $\Phi_{u} \in C\left(\left[0, \omega_{M}\right], X\right)$. Mostremos que $\Phi_{u} \in E$. Para $t \in\left[0, \omega_{M}\right]$, temos que $\|u(t)\| \leq$ $K$ e portanto

$$
\begin{aligned}
\|F(u(t))\| & \leq\|F(0)\|+\|F(u(t))-F(0)\| \leq\|F(0)\|+L_{K}\|u(t)-0\| \\
& \leq\|F(0)\|+K L_{K}=\|F(0)\|+2 M \alpha L_{K}+\|F(0)\| L_{K} \\
& \leq 2\|F(0)\|+2 M \alpha L_{K}+2\|F(0)\| L_{K} 2 M \alpha=\left(2 L_{K}+2\right)(M \alpha+\|F(0)\|) .
\end{aligned}
$$

Como $\omega_{M} \leq \tau$, obtemos para $t \in\left[0, \omega_{M}\right]$ que

$$
\begin{aligned}
\left\|\Phi_{u}(t)\right\| & \leq\|T(t)\|\|x\|+\int_{0}^{t}\|T(t-s)\|\|F(u(s))\| d s \\
& \leq \alpha M+\alpha\left(2+2 L_{K}\right)(M \alpha+\|F(0)\|) t \leq \alpha M+\alpha\left(2+2 L_{K}\right)(M \alpha+\|F(0)\|) \omega_{M} .
\end{aligned}
$$

Como $\omega_{M} \leq \frac{1}{\left(2 L_{K}+2\right) \alpha}$ temos que

$$
\left\|\Phi_{u}(t)\right\| \leq 2 M \alpha+\|F(0)\|=K, \text { para todo } t \in\left[0, \omega_{M}\right] .
$$


Portanto, temos definida uma aplicação $F: E \rightarrow E$ dada por $F(u)=\Phi_{u}, u \in E$. Além disso, para todo $u, v \in E$, temos

$$
\left\|\Phi_{v}(t)-\Phi_{u}(t)\right\| \leq L_{K} \int_{0}^{t}\|T(t)\|\|v(s)-u(s)\| d s \leq \alpha \omega_{M} L_{K} d(u, v), t \in\left[0, \omega_{M}\right]
$$

Suponha que para $n \in \mathbb{N}, n>1$ temos que

$$
\left\|\Phi_{u}^{n-1}(t)-\Phi_{v}^{n-1}(t)\right\| \leq \frac{\left(\alpha L_{K} \omega_{M}\right)^{n-1}}{(n-1) !} d(u, v), t \in\left[0, \omega_{M}\right]
$$

Para todo $t \in\left[0, \omega_{M}\right]$ segue que

$$
\begin{aligned}
\left\|\Phi_{u}^{n}(t)-\Phi_{v}^{n}(t)\right\| & \leq \int_{0}^{t} \frac{\left(\alpha L_{K}|s|\right)^{n-1}}{(n-1) !}\|T(t-s)\|\left\|\Phi_{u}(t)-\Phi_{v}(t)\right\| d(u, v) \\
& \leq \alpha^{n} L_{K}^{n} d(u, v) \int_{0}^{t} \frac{|s|^{n-1}}{(n-1) !} d s=\frac{\left(\alpha L_{K} \omega_{M}\right)^{n}}{n !} d(u, v) .
\end{aligned}
$$

Portanto, mostramos que para todo $n \in \mathbb{N}$ temos que

$$
\left\|\Phi_{u}^{n}(t)-\Phi_{v}^{n}(t)\right\| \leq \frac{\left(\alpha L_{K} \omega_{M}\right)^{n}}{n !} d(u, v), \text { para todo } t \in\left[0, \omega_{M}\right]
$$

Logo, para todo $n \in \mathbb{N}$

$$
d\left(F^{n}(u), F^{n}(v)\right) \leq \frac{\left(\alpha L_{K} \omega_{M}\right)^{n}}{n !} d(u, v) .
$$

Seja $n \in \mathbb{N}$ suficientemente grande tal que

$$
\frac{\left(\alpha L_{K} \omega_{M}\right)^{n}}{n !}<1
$$

Logo, $F^{n}$ é uma contração em $E$. Assim, segue do Teorema do Ponto Fixo de Banach, a aplicação $F^{n}$ possui um único ponto fixo $u \in E$ e, portanto, o mesmo ocorre para $F$. O único ponto fixo da aplicação $F$ é uma solução $u \in C\left(\left[0, \omega_{M}\right], X\right)$ de (1.4) em $\left[0, \omega_{M}\right]$.

Para concluir mostremos a unicidade. Sejam $u, v \in C\left(\left[0, \omega_{M}\right], X\right)$ soluções de (1.4) em $\left[0, \omega_{M}\right]$ e defina $P=\sup _{t \in\left[0, \omega_{M}\right]}\{\|u(t)\|,\|v(t)\|\}$. Para $t \in\left[0, \omega_{M}\right]$ temos que

$$
\|u(t)-v(t)\| \leq \int_{0}^{t}\|F(u(s))-F(v(s))\| d s \leq L_{P} \int_{0}^{t}\|u(s)-v(s)\| d s .
$$

O Lema $1.61\left(\operatorname{com} C_{1}=0\right)$ implica que $u(t)=v(t)$, para todo $t \in\left[0, \omega_{M}\right]$. 
Demonstração do Teorema 1.63 Seja $x \in X$. Defina o seguinte conjunto

$$
\mathscr{C}:=\{\omega>0 \mid \text { existe uma única solução } u \in C([0, \omega], X) \text { de }[1.4) \text { em }[0, \omega]\} .
$$

Segue da Proposição 1.62 que

$$
\omega(x)=\sup \mathscr{C}
$$

está bem definido e $\omega(x) \in(0, \infty]$. Seja $t \in[0, \infty)$. Logo existe um $T \in \mathscr{C}$ tal que $0<t \leq T$. Seja $u_{T} \in C([0, T], X)$ a única solução de $(1.4)$ em $[0, T]$ e defina

$$
u(t):=u_{T}(t)
$$

Afimamos que a função $u$ está bem definida. De fato, seja $S \in \mathscr{C}$ com $0<t \leq S$. Sem perda de generalidade podemos supor que $S<T$. Logo $u_{S}$ e $u_{T}$ são duas soluções de (1.4) definidas em $[0, S]$. A unicidade da Proposição 1.62 implica que $u_{S}=u_{T}$ em $[0, S]$ e a afirmativa está demonstrada.

Além disso, a função $u$ é contínua em $[0, \omega(x))$ e para todo para todo $0<\omega<\omega(x)$, $u$ é a única solução de (1.4) em $C([0, \omega], X)$.

Para completar a demonstração do resultado, se $\omega(x)<\infty$, mostremos que $\lim _{t \rightarrow \omega(x)^{-}}\|u(t)\|=\infty$. Suponha que exista um $M \geq 0$ tal que $\|u(t)\| \leq M$ para todo $t \in[0, \omega(x))$ e seja $\omega_{M}$ como na Proposição 1.62 . Temos que $\omega_{M}<\omega(x)$. Seja $\left.\left.t_{0} \in\right] 0, \omega(x)\right)$ tal que

$$
\omega(x)-t_{0}<\omega_{M}
$$

Seja $v \in C\left(\left[0, \omega_{M}\right], X\right)$ a solução de (1.4) dada pela Proposição 1.62, Logo

$$
v(s)=T(s) u\left(t_{0}\right)+\int_{0}^{s} T(s-\sigma) F(v(\sigma)) d \sigma, \text { para todo } s \in\left[0, \omega_{M}\right] .
$$

Defina $w \in C\left(\left[0, t_{0}+\omega_{M}\right], X\right)$ por

$$
w(s)= \begin{cases}u(s) & \text { se } s \in\left[0, t_{0}\right] \\ v\left(s-t_{0}\right) & \text { se } s \in\left[t_{0}, t_{0}+\omega_{M}\right] .\end{cases}
$$

Afirmamos que $w$ é solução de (1.4) com $\omega=t_{0}+\omega_{M}$. De fato, para $\tau \in\left[0, t_{0}\right]$ temos que

$$
w(\tau)=u(\tau)=T(\tau) x+\int_{0}^{t_{0}} T\left(t_{0}-s\right) F(u(s)) d s=T(\tau) x+\int_{0}^{t_{0}} T\left(t_{0}-s\right) F(w(s)) d s .
$$


Agora, para $\tau \in\left[t_{0}, t_{0}+\omega_{M}\right]$ temos que

$$
\begin{aligned}
& w(\tau)=v\left(\tau-t_{0}\right)=T\left(\tau-t_{0}\right) u\left(t_{0}\right)+\int_{0}^{\tau-t_{0}} T\left(\tau-t_{0}-\sigma\right) F(v(\sigma)) d \sigma \\
& \quad=T\left(\tau-t_{0}\right)\left(T\left(t_{0}\right) x+\int_{0}^{t_{0}} T\left(t_{0}-\sigma\right) F(u(\sigma)) d \sigma\right)+\int_{0}^{\tau-t_{0}} T\left(\tau-t_{0}-\sigma\right) F(v(\sigma)) d \sigma \\
& \quad=T(\tau) x+\int_{0}^{t_{0}} T(\tau-\sigma) F(u(\sigma)) d \sigma+\int_{t_{0}}^{\tau} T(\tau-\sigma) F\left(v\left(\sigma-t_{0}\right)\right) d \sigma \\
& \quad=T(\tau) x+\int_{0}^{\tau} T(\tau-\sigma) F(w(\sigma)) d \sigma .
\end{aligned}
$$

O que demonstra a afirmativa. A definição de $\omega(x)$ implica que $t_{0}+\omega_{M} \leq \omega(x)$, mas isso contradiz A.8. Logo o conjunto $\{\|u(t)\| \mid t \in[0, \omega(x))\}$ não é limitado.

Agora suponha que exista uma constante $M \geq 0$ tal que para todo $\tau \in[0, \omega(x))$ exista um $t_{\tau} \in(\tau, \omega(x))$ tal que $\left\|u\left(t_{\tau}\right)\right\| \leq M$. Em particular, para todo $n \in \mathbb{N}$ existe um $t_{n} \in(\omega(x)-$ $1 / n, \omega(x))$ tal que $\left\|u\left(t_{n}\right)\right\| \leq M$. Temos que $t_{n} \rightarrow \omega(x)^{-}$quando $n \rightarrow \infty$. Seja $n_{0} \in \mathbb{N}$ tal que $\omega(x)-t_{n_{0}}<\omega_{M}$. Usando um argumento similar ao acima, mostramos que $\omega_{M}+t_{n_{0}}<\omega(x)$ o que é uma contradição. Portanto, $\lim _{t \rightarrow \omega(x)^{-}}\|u(t)\|=\infty$.

Demonstração da Proposição 1.64 Seja $x \in X$ e seja $u$ solução da equação (1.4) dada pelo Teorema 1.63 . Seja $0<\omega<\omega(x)$. Seja $\left(x_{n}\right)_{n}$ uma sequência em $X$ tal que $x_{n} \rightarrow x \operatorname{com} n \rightarrow \infty$. Para cada $n \in \mathbb{N}$ seja $u_{n}$ a solução da equação (1.4) dada pelo Teorema 1.63 correspondente ao valor inicial $x_{n}$. Como na demonstração do Teorema 1.63 seja $\alpha=\max \{\|T(t)\| \mid t \in[0,1]\}$. Afirmamos que existe um $n_{0} \in \mathbb{N}$ tal que $\omega\left(x_{n}\right)>\omega$ para todo $n \geq n_{0}$. Para ver isto, defina

$$
M=\alpha^{-1} \sup _{t \in[0, \omega]}\|u(t)\|
$$

e para cada $n \in \mathbb{N}$ defina

$$
\tau_{n}=\sup \left\{t \in\left[0, \omega\left(x_{n}\right)\right) \mid\left\|u_{n}(s)\right\| \leq 2 M \alpha+\|F(0)\|, \text { para todo } s \in[0, t]\right\}
$$

Como a sequência $\left(x_{n}\right)_{n}$ é convergente, então existe um $n_{1} \in \mathbb{N}$ tal que $\left\|x_{n}\right\|<M$ para todo $n \geq n_{1}$. É claro que $\omega_{M}<\omega\left(x_{n}\right)$ e $\tau_{n}>\omega_{M}>0$. Defina $\beta=\max \{\|T(t)\| \mid t \in[0, \omega]\}$. Para cada $t \leq \omega, t \leq \tau_{n}$, temos

$$
\begin{aligned}
\left\|u(t)-u_{n}(t)\right\| & \leq\|T(t)\|\left\|x-x_{n}\right\|+L_{2 M \alpha+\|F(0)\|} \int_{0}^{t}\|T(t-s)\|\left\|u(s)-u_{n}(s)\right\| d s \\
& \leq \beta\left\|x-x_{n}\right\|+\beta L_{2 M \alpha+\|F(0)\|} \int_{0}^{t}\left\|u(s)-u_{n}(s)\right\| d s .
\end{aligned}
$$


Segue da Desigualdade de Gronwall descrita no Lema 1.61 que

$$
\left\|u(t)-u_{n}(t)\right\| \leq \beta\left\|x-x_{n}\right\| e^{\omega \beta L_{2 M \alpha+\|F(0)\|}} \text {, para todo } t \leq \omega, t \leq \tau_{n} .
$$

Seja $n_{0} \in \mathbb{N} \operatorname{com} n_{0} \geq n_{1}$ tal que

$$
\left\|x-x_{n}\right\| \leq \frac{M \alpha}{\beta e^{\omega \beta L_{2 M \alpha+\|F(0)\|}}}, \text { para todo } n \geq n_{0} .
$$

Em particular, para todo $t \leq \min \left\{\omega, \tau_{n}\right\}$ e $n \geq n_{0}$, temos que

$$
\left\|u_{n}(t)\right\| \leq\|u(t)\|+\beta\left\|x-x_{n}\right\| e^{\omega \beta L_{2 M \alpha+\|F(0)\|}} \leq M \alpha+M \alpha \leq 2 M \alpha+\|F(0)\| .
$$

Logo, $\tau_{n}>\min \left\{\omega, \tau_{n}\right\}$ para todo $n \geq n_{0}$. Ou seja, $\tau_{n}>\omega$ para todo $n \geq n_{0}$ e isso implica que $\omega\left(x_{n}\right)>\omega$ para todo $n \geq n_{0}$. A demonstração da afirmativa está completa.

Notemos que a semicontinuidade inferior de $\omega$ segue imediatamente da afirmativa acima. Para demonstrar (ii) notemos que se $\left(x_{n}\right)_{n}$ é uma sequência em $X$ e $x \in X$ tais que $x_{n} \rightarrow x$ quando $n \rightarrow \infty$ e se $\omega<\omega(x)$, então pelo que fizemos acima temos que existe uma constante $\widetilde{K} \geq 0$ tal que para $n \in \mathbb{N}$ suficientemente grande temos que $\omega\left(x_{n}\right)>\omega \mathrm{e}$

$$
\left.\left.\left\|u(t)-u_{n}(t)\right\| \leq \widetilde{K}\left\|x-x_{n}\right\|, \text { para todo } t \in\right] 0, \omega\right]
$$

onde para cada $n \in \mathbb{N}$ sejam $u_{n}$ e $u$ as soluções da equação (1.4) correspondentes aos valores iniciais $x_{n}$ e $x$. A desigualdade (A.10) implica que $u_{n} \rightarrow u$ em $C([0, \omega], X)$. 


\section{Referências Bibliográficas}

[1] J.M. Arrieta, J.W. Cholewa, T. Dłtoko e A. Rodrigues-Benal, Asymptotoic behavior and attractors for reaction-diffusion equations in unbounded domains, Nonlinear Anal. 56 (2004), 515-554.

[2] A.V. Babin e M.I. Vishik, Attractor of Evolution Equations, North Holland, Amsterdam, 1991.

[3] A.V. Babin e M.I. Vishik, Attractor of partial differential evolution equations in an unbounded domain, Proc. Roy. Soc. Edinburgh Sect. A 116 (1990), 221-243.

[4] J.M. Ball, Global attractors for damped semilinear wave equations. Partial differential equations and applications, Discrete Contin. Dynam. Systems 10 (2004), 31-52.

[5] H. Brézis, Functional Analysis, Sobolev Spaces and Partial Differential Equations, Universitext, Springer, New York, 2011.

[6] A.N. Carvalho, Análise Funcional II, Notas de Aula. Modelo de documento digital disponível em <http://www2.icmc.usp.br/ andcarva/AnaliseFuncional-II.pdf $>$. Acesso em: 13 de dezembro de 2012.

[7] A.N. Carvalho, Sistemas Dinâmicos Não-Lineares, Notas de Aula. Modelo de documento digital disponível em < http://www2.icmc.usp.br/ andcarva/SDNL2012.pdf > . Acesso em: 13 de dezembro de 2012.

[8] T. Cazenave e A. Haraux, An Introduction to Semilinear Evolution Equations, Oxford Science Publications, Oxford, 1998.

[9] J.W. Cholewa e T. Dłtoko, Global Attractors in Abstract Parabolic Problems, Cambridge University Press, Cambridge, 2000.

[10] R. Dautray e J.L. Lions, Mathematical Analysis and Numerical Methods for Science and Technology - Volume 2, Springer-Verlag, Berlin, 1988. 
[11] K. Deimling, Nonlinear Functional Analysis, Springer-Verlag, 1985.

[12] N. Dunford e J.T. Schwartz, Linear Operators, Part I, Interscience Publishers, New York, 1958.

[13] D. Fall e Y. You, Global attractors for the damped nonlinear wave equation in unbounded domin, Proceedings of the Fourth World Congress of Nonlinear Analysis (2004).

[14] E. Feireisl, Attractors for semilinear damped wave equations on $\mathbb{R}^{3}$, Nonlinear Anal. 23 (1994). 187-195.

[15] E. Feireisl, Asymptotic behaviour and attractors for semilinear damped wave equaitons with a supercritical exponent, Proc. Roy. Soc. Edinburgh Sect. A 125 (1995), 1051-1062.

[16] D.G. Figueiredo, The Ekeland Variational Principle with Applications and Detours, Springer-Verlag, Berlin, 1989.

[17] J.A. Goldstein, Semigroups of Linear Operators and Applications, Oxford Mathematical Monographs, Oxford University Press, New York, 1985.

[18] J. Hale, Asymptotic Behavior of Dissipative Systems, American Mathematical Society, Providence, 1988.

[19] J. Hale e G. Rangel, Upper semicontinuity of the attractor for a singularly perturbed hyperbolic equation, J. Differetial Equations. 73 (1988), 197-214.

[20] D. Henry, Geometric Theory of Semilinear Parabolic Equations, Springer-Verlag, Berlin, 1981.

[21] E. Hille e R.S. Phillips, Functional Analysis and Semigroups, AMS, Providence, 1957.

[22] O. Ladyzenskaya, Attractors for Semigroups and Evolution Equations, Cambridge University Press, Cambridge, 1991.

[23] A. Pazy, Semigroups of Linear Operators and Applications to Partial Differential Equations, Springer-Verlag, New York, 1983.

[24] M. Prizzi e K. P. Rybakowski, Attractors for singularly perturbed damped wave equations on arbitrary unbounded domains, Top. Meth. in Nonlinear Anal. 32 (2008), 1-20.

[25] M. Prizzi e K. P. Rybakowski, Attractors for semilinear damped wave equations on arbitrary unbounded domains, Top. Meth. in Nonlinear Anal. 31 (2008), 49-82. 
[26] M. Prizzi e K. P. Rybakowski, Attractors for reaction-diffusion equations on arbitrary unbounded domains, Top. Meth. in Nonlinear Anal. 30 (2007), 251-277.

[27] B. Simon, Schrödinger Semigroups, Bull. AMS. 7 (1982) 447-526.

[28] R. Temam, Infinite Dimensional Dynamical Systems in Mechanics and Physics, SpringerVerlag, New York, 1997.

[29] B. Wang, Attractors for reaction-diffusion equations in unbounded domains, Physica D 179 (1999), 41-52. 